\title{
Modeling and Analysis of Different Aspects of Finite-Rate Chemical Kinetics in Hypersonic Combustors
}

\author{
A Dissertation \\ Presented to the Faculty of the \\ School of Engineering and Applied Science \\ University of Virginia
}

In Partial Fulfillment

of the Requirements for the Degree of

Doctor of Philosophy in

Mechanical and Aerospace Engineering

by

Mohammad Javad Rahimi

August 2016 


\section{Approval Sheet}

This dissertation is submitted in partial fulfillment of the requirements for the degree of Doctor of Philosophy in Mechanical and Aerospace Engineering

Mohammad Javad Rahimi

This dissertation has been read and approved by the examining Committee:

Prof. Harsha K. Chelliah, Dissertation Advisor

Prof. James C. McDaniel

Prof. Houston G. Wood

Prof. Christopher P. Goyne

Prof. Robert J. Davis

Accepted for the School of Engineering and Applied Science:

Craig H. Benson, Dean, School of Engineering and Applied Science

August 2016 


\section{Abstract}

Hypersonic air-breathing propulsion systems offer more efficient access to space compared to traditional rocket based propulsion systems due to their higher specific impulse and reusability. However, actual implementation of hypersonic air-breathing engine is hampered by a number of technical challenges. First, a vehicle operating at high speeds (Mach 5 and above) will experience extremely high temperatures especially at the combustor walls. One proposed concept is to use the on board fuel itself for active cooling of the hot surfaces which results in significant reduction of the weight of the vehicle by eliminating the need for carrying cooling fluids and heat exchanger.

When hydrocarbon fuels are exposed to high temperatures in cooling channels, they not only absorb heat physically (sensible heat), but also crack to smaller hydrocarbons and absorb chemical heat. This process is called endothermic pyrolysis which has two main benefits. First, it increases the cooling capacity of the fluid beyond its sensible heat by nearly a factor of two. Second, the cracked fuel components produced from the pyrolysis reduce the induction time of the fuel-air mixture in the combustor, which is an important parameter in hypersonic flights with short flow residence times.

In the present work, endothermic pyrolysis of different jet fuels occurring in such cooling channels is explored under experimental conditions performed in the UVa high-pressure flow reactor. The analysis presented includes modeling of finite rate chemical kinetics and multidimensional transport effects in representative cooling channels. The present results show significant differences exist between the currently available finite-rate chemical kinetic models. The sensitivity analysis, reaction path flux analysis, and uncertainty quantification of the chemical kinetic models in this work, provide guide lines for improvement and optimization of kinetic model parameters.

The second major challenge is maintaining combustion at hypersonic flow condi- 
tions where the available time for the fuel injection, mixing, ignition, and complete combustion is less than a millisecond. University of Virginia Supersonic Combustion Facility (UVaSCF) is currently exploring parametric conditions using a cavity flame holder to address certain aspects of these challenges. The complexity of the turbulent reacting flow in the combustor makes parametric study with full 3D CFD modeling computationally expensive. In this work a multiple reactor network including partially stirred reactor, perfectly stirred reactor, and closed homogeneous reactor model is developed to address the key effects of both flow residence time and turbulence mixing time scales on the flame holding and stabilization limits.

The third challenge that is addressed in this work, is the thermal choking in dualmode hypersonic combustor. A novel computational model is developed in this work to predict the thermal choking in a variable area combustor. This tool provides a computationally efficient model to predict the operability limits of hypersonic combustors at different flight conditions without the need for time consuming multi-dimensional reacting flow simulations.

As part of the present work, reduced chemical kinetic models are also developed for reacting flow simulations in hypersonic applications. A reduced kinetic model for ethylene combustion and a reduced kinetic model for n-dodecane pyrolysis were extracted using Principal component analysis. In addition, a one-step lumped reaction model is developed for the pyrolysis of JP-8 aviation fuel to major smaller hydrocarbon species. The accuracy of these reduced order models were tested by comparing their predictions with experimental data and detailed chemistry mechanisms.

The fundamental analysis performed to understand various rate controlling reactions contributing to fuel pyrolysis in cooling channels and ignition phenomena in turbulent flame holding regimes are expected to advance design of future hypersonic vehicles. 


\section{Acknowledgements}

First, I must thank my family, who supported me during all my educational and academic stages. My parents always encouraged me in my studies and admired my passion to pursue my scientific goals. I would like to thank my wife Mehrsa for all the beautiful things she has given me in the last few years. Her support and encouragement made my life as a graduate student the most enjoyable experience.

I would like to thank my advisor, Professor Harsha Chelliah, without whom this dissertation would not have been possible. He has provided me with great knowledge and challenged me to become the best researcher I can be. For his support, patience, and dedication, I am forever in his debt. Thanks are due as well to my committee members Professor Wood, Professor McDaniel, Professor Goyne, and Professor Davis for their time and guidance.

Many thanks are due as well to my colleagues in the Combustion and Energy Laboratory at the University of Virginia. My good friend and colleague Dr. Ryan Johnson was with me from the beginning. We studied together, did research and programming together, and I always enjoyed having him beside me in the Lab. When I began working in the lab, Dr. Gaetano Esposito taught me how to perform combustion modeling with detailed chemistry and perform mechanism reduction. He was a great friend and mentor that I greatly miss the time I have spent with him. I also thank my friends and colleagues Ujuma Shrestha and Gregory Simms. We spent a great amount of time discussing the research problems regarding our tube reactor experiment. Many thanks to my other friends in the Combustion and Energy Lab for being such a good company in the last few years as a PhD student. 


\section{Contents}

1 Introduction $\quad 2$

1.1 Hypersonic Airbreathing Propulsion . . . . . . . . . . . . . . . 2

1.2 Objectives of this Work . . . . . . . . . . . . . 5

1.3 Overview . . . . . . . . . . . . . . . . . 8

2 Endothermic Fuel Pyrolysis in Cooling Channels 11

2.1 Background ...................... . . 11

2.2 Experimental Methods . . . . . . . . . . . . . . . 16

2.2.1 Introduction to Flow Reactors . . . . . . . . . . . . . 16

2.2.2 UVa Micro Flow Tube Reactor . . . . . . . . . . . . . . . . 18

2.3 Governing Equations . . . . . . . . . . . . . . . . 20

2.3.1 Zero-Dimensional Model Governing Equations . . . . . . . . . 20

2.3.2 One-Dimensional Model Governing Equations . . . . . . . . . 21

2.4 Taylor Aris Dispersion . . . . . . . . . . . . . . . . . . 22

2.5 Detailed Chemical Kinetic Models . . . . . . . . . . . . . . . . 24

2.5.1 Detailed Mechanisms used for Model Prediction . . . . . . . . 25

2.6 Model Results Comparison with Experiment . . . . . . . . . . . . 28

2.6.1 Ethane . . . . . . . . . . . . . . . . 28

2.6 .2 n-Butane . . . . . . . . . . . . . . . . . . . . . . . . . . . . . . . . . .

2.6 .3 n-Dodecane . . . . . . . . . . . . . . . . . 46

2.7 JP-8 Pyrolysis Lumped Model . . . . . . . . . . . . . . . . . . . . 59

2.8 Summary . . . . . . . . . . . . . . . . . 65

3 Chemical Kinetic Uncertainty Analysis of Endothermic Fuel Pyrol$\begin{array}{ll}\text { ysis } & 67\end{array}$

3.1 Sensitivity Analysis . . . . . . . . . . . . . . . . . . . 68

3.2 Reaction Pathway Analysis . . . . . . . . . . . . . . . . . . . . 71

3.3 Model Uncertainty Analysis . . . . . . . . . . . . . . . . . . 76

3.4 Mechanism Reduction for n-Dodecane Pyrolysis . . . . . . . . . . . . 90

3.5 Ethane Pyrolysis . . . . . . . . . . . . . . . . . . . . . . . . 96

3.6 n-Butane Pyrolysis . . . . . . . . . . . . . . . . . . . . . . . . 104

3.7 Summary . . . . . . . . . . . . . . . . . . . . . 111 


\section{Two-Dimensional Axisymmetric Simulation of a Tubular Cooling}

Channel 113

4.1 Two-dimensional Model Governing Equations . . . . . . . . . . . . 114

4.2 Geometry and Grid Resolution . . . . . . . . . . . . . . 116

4.3 Boundary and Initial Conditions . . . . . . . . . . . . . . . 117

4.4 Two-dimensional Axi-symmetric Simulation Result . . . . . . . . . 117

4.4 .1 Contour Plots . . . . . . . . . . . . . . . . . . . . 117

$4.4 .2 \quad$ Radial Variation Plots . . . . . . . . . . . . . . . . . . . . . . 122

4.4 .3 Axial Variation Plots . . . . . . . . . . . . . . . . . . . . . . . 129

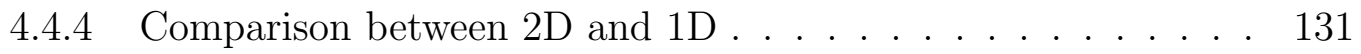

4.5 Summary . . . . . . . . . . . . . . . . . . 137

5 Chemical Analysis of Fuel Oxidation in Supersonic Combustor 139

5.1 Turbulence and Combustion . . . . . . . . . . . . . . . . . . . 139

5.2 Partially Stirred Reactor (PaSR) analysis . . . . . . . . . . . . . . 142

5.2.1 Introduction and Description of PaSR . . . . . . . . . . . 142

5.2.2 Supersonic Combustion Reactor Configuration . . . . . . . . . 145

5.2.3 Reactor Flow Characteristic Time Scales . . . . . . . . . . . 148

5.2 .4 PaSR Modeling Conditions . . . . . . . . . . . . . . . . . . . . 149

5.2 .5 PaSR Results . . . . . . . . . . . . . . . . . . . . 153

5.3 Ignition Delay Time Analysis . . . . . . . . . . . . . 156

5.4 Mechanism Reduction Based on PaSR . . . . . . . . . . . . . . 161

5.4.1 Ethylene Combustion Reduced Model . . . . . . . . . . . . . . 161

5.4.2 Reduced Model Validation and Performance . . . . . . . . . . 163

5.5 Summary . . . . . . . . . . . . . . . . . . 165

6 Thermal Choking in Hypersonic Combustor 167

6.1 Rayleigh Flow . . . . . . . . . . . . . . . . . . . . . 168

6.2 Formulation of the Thermal Choking in a Variable Area . . . . . . . 170

6.2.1 Quasi-One-Dimensional Flow Equations . . . . . . . . . . 170

6.2.2 Energy Conservation Equation in a Variable Area Thermally

Choked Flow . . . . . . . . . . . . . . . . . . . . 174

6.3 Results and Discussion . . . . . . . . . . . . . . . . 177

6.4 Summary . . . . . . . . . . . . . . . . . . . 180

$\begin{array}{lll}7 & \text { Summary and Future Works } & 181\end{array}$

A Reduced Ethylene-Air Reaction Mechanism 185

B Reduced n-Dodecane Pyrolysis Reaction Mechanism 198 


\section{List of Figures}

1.1 Performance (specific impulse) of air-breathing systems versus rockets as a function of flight Mach number [2]. . . . . . . . . . . . . . . . . . 3

1.2 Turbine-based combined cycle hypersonic vehicle concept [4] . . . . . 5

1.3 Schematic of the hypersonic combustor. Three research focuses in this work are: (1) Engine cooling using fuel endothermic pyrolysis, (2) Chemical analysis of the fuel oxidation in the combustor, (3) Thermal choking and operability limits of hypersonic combustor. . . . . . 6

2.1 Heat transfer in the control volume of the cooling channel. . . . . . . 13

2.2 Heat sink for n-dodecane pyrolysis. . . . . . . . . . . . . . . . . . . 14

2.3 Schematic of an idealized plug flow reactor with species profiles as a function of the axial coordinate. . . . . . . . . . . . . . . . 17

2.4 Schematic of UVa Micro-Flow Tube Reactor setup. Diluent is preheated in the helical section to the desired temperature. The fuel is added through the side tubes and mixes with the preheated gas and reacts along the tube with a near constant temperature variation. A quartz probe is used to transfer a sample to the gas analysis system. .

2.5 Nondimensionalized effective dispersion coefficient as a function of Peclet number (the minimum occurs at $P e=6.9$ ) . . . . . . . . . . . . .

2.6 Ethane pyrolysis in $1 \mathrm{D}$ flow reactor simulation with and without TaylorAris dispersion coefficient. . . . . . . . . . . . . . .

2.7 Species mole fraction as a function of temperature for $2 \%$ ethane pyrolysis in the atmospheric pressure tube reactor at $10 \mathrm{~ms}$ residence time. Symbols are the experimental data with error bar indicating the uncertainty of the data measurements. Lines are the model prediction using the three different chemistry mechanisms. . . . . . . . . . .

2.8 Species mole fraction as a function of temperature for $2 \%$ ethane pyrolysis in the atmospheric pressure tube reactor at $10 \mathrm{~ms}$ residence time. Symbols are the experimental data with error bar indicating the uncertainty of the data measurements. Lines are the model prediction using the three different chemistry mechanisms. . . . . . . . . . .

2.9 Species mole fraction as a function of temperature for $2 \%$ ethane pyrolysis in the atmospheric pressure tube reactor at $50 \mathrm{~ms}$ residence time. Symbols are the experimental data with error bar indicating the uncertainty of the data measurements. Lines are the model prediction using the three different chemistry mechanisms. . . . . . . . . . 
2.10 Species mole fraction as a function of temperature for $2 \%$ ethane pyrolysis in the atmospheric pressure tube reactor at $50 \mathrm{~ms}$ residence time. Symbols are the experimental data with error bar indicating the uncertainty of the data measurements. Lines are the model prediction using the three different chemistry mechanisms. . . . . . . . . . .

2.11 Species mole fraction as a function of temperature for $2 \%$ ethane pyrolysis in the atmospheric pressure tube reactor at $70 \mathrm{~ms}$ residence time. Symbols are the experimental data with error bar indicating the uncertainty of the data measurements. Lines are the model prediction using the three different chemistry mechanisms. . . . . . . . . . .

2.12 Species mole fraction as a function of temperature for $2 \%$ ethane pyrolysis in the atmospheric pressure tube reactor at $70 \mathrm{~ms}$ residence time. Symbols are the experimental data with error bar indicating the uncertainty of the data measurements. Lines are the model prediction using the three different chemistry mechanisms. . . . . . . . . . .

2.13 Species mole fraction as a function of temperature for $2 \%$ ethane pyrolysis in the atmospheric pressure tube reactor at $90 \mathrm{~ms}$ residence time. Symbols are the experimental data with error bar indicating the uncertainty of the data measurements. Lines are the model prediction using the three different chemistry mechanisms. . . . . . . . . . . .

2.14 Species mole fraction as a function of temperature for $2 \%$ ethane pyrolysis in the atmospheric pressure tube reactor at $90 \mathrm{~ms}$ residence time. Symbols are the experimental data with error bar indicating the uncertainty of the data measurements. Lines are the model prediction using the three different chemistry mechanisms. . . . . . . . . . .

2.15 Species mole fraction as a function of temperature for $2 \%$ n-butane pyrolysis in the atmospheric pressure tube reactor at $30 \mathrm{~ms}$ residence time. Symbols are the experimental data with error bar indicating the uncertainty of the data measurements. Lines are the model prediction using the three different chemistry mechanisms. . . . . . . . . . .

2.16 Species mole fraction as a function of temperature for $2 \%$ n-butane pyrolysis in the atmospheric pressure tube reactor at $30 \mathrm{~ms}$ residence time. Symbols are the experimental data with error bar indicating the uncertainty of the data measurements. Lines are the model prediction using the three different chemistry mechanisms. . . . . . . . . . .

2.17 Species mole fraction as a function of temperature for $2 \%$ n-butane pyrolysis in the atmospheric pressure tube reactor at $50 \mathrm{~ms}$ residence time. Symbols are the experimental data with error bar indicating the uncertainty of the data measurements. Lines are the model prediction using the three different chemistry mechanisms. . . . . . . . . . . .

2.18 Species mole fraction as a function of temperature for $2 \%$ n-butane pyrolysis in the atmospheric pressure tube reactor at $50 \mathrm{~ms}$ residence time. Symbols are the experimental data with error bar indicating the uncertainty of the data measurements. Lines are the model prediction using the three different chemistry mechanisms. . . . . . . . . . 
2.19 Species mole fraction as a function of temperature for $2 \%$ n-butane pyrolysis in the atmospheric pressure tube reactor at $70 \mathrm{~ms}$ residence time. Symbols are the experimental data with error bar indicating the uncertainty of the data measurements. Lines are the model prediction using the three different chemistry mechanisms. . . . . . . . . . .

2.20 Species mole fraction as a function of temperature for $2 \%$ n-butane pyrolysis in the atmospheric pressure tube reactor at $70 \mathrm{~ms}$ residence time. Symbols are the experimental data with error bar indicating the uncertainty of the data measurements. Lines are the model prediction using the three different chemistry mechanisms. . . . . . . . . . .

2.21 Species mole fraction as a function of temperature for $2 \%$ n-butane pyrolysis in the atmospheric pressure tube reactor at $90 \mathrm{~ms}$ residence time. Symbols are the experimental data with error bar indicating the uncertainty of the data measurements. Lines are the model prediction using the three different chemistry mechanisms. . . . . . . . . . .

2.22 Species mole fraction as a function of temperature for $2 \%$ n-butane pyrolysis in the atmospheric pressure tube reactor at $90 \mathrm{~ms}$ residence time. Symbols are the experimental data with error bar indicating the uncertainty of the data measurements. Lines are the model prediction using the three different chemistry mechanisms. . . . . . . . . . .

.23 Species mole fraction as a function of temperature for $0.25 \% \mathrm{n}$-Dodecane pyrolysis in the atmospheric pressure tube reactor at $10 \mathrm{~ms}$ residence time. Symbols are the experimental data with error bar indicating the uncertainty of the data. Lines are the model prediction using four different chemistry mechanisms. . . . . . . . . . . . . . .

2.24 Species mole fraction as a function of temperature for $0.25 \% \mathrm{n}$-dodecane pyrolysis in the atmospheric pressure tube reactor at $10 \mathrm{~ms}$ residence time. Symbols are the experimental data with error bar indicating the uncertainty of the data. Lines are the model prediction using four different chemistry mechanisms. . . . . . . . . . . . . . .

2.25 Species mole fraction as a function of temperature for $0.25 \% \mathrm{n}$-Dodecane pyrolysis in the atmospheric pressure tube reactor at $20 \mathrm{~ms}$ residence time. Symbols are the experimental data with error bar indicating the uncertainty of the data. Lines are the model prediction using four different chemistry mechanisms. . . . . . . . . . . . . .

2.26 Species mole fraction as a function of temperature for $0.25 \%$ n-dodecane pyrolysis in the atmospheric pressure tube reactor at $20 \mathrm{~ms}$ residence time. Symbols are the experimental data with error bar indicating the uncertainty of the data. Lines are the model prediction using four different chemistry mechanisms. . . . . . . . . . . . . . .

2.27 Species mole fraction as a function of temperature for $0.25 \% \mathrm{n}$-Dodecane pyrolysis in the atmospheric pressure tube reactor at $30 \mathrm{~ms}$ residence time. Symbols are the experimental data with error bar indicating the uncertainty of the data. Lines are the model prediction using four different chemistry mechanisms. . . . . . . . . . . . 
2.28 Species mole fraction as a function of temperature for $0.25 \% \mathrm{n}$-dodecane pyrolysis in the atmospheric pressure tube reactor at $30 \mathrm{~ms}$ residence time. Symbols are the experimental data with error bar indicating the uncertainty of the data. Lines are the model prediction using four different chemistry mechanisms. . . . . . . . . . . . . . . . .

2.29 Species mole fraction as a function of temperature for $0.25 \%$ n-Dodecane pyrolysis in the atmospheric pressure tube reactor at $40 \mathrm{~ms}$ residence time. Symbols are the experimental data with error bar indicating the uncertainty of the data. Lines are the model prediction using four different chemistry mechanisms. . . . . . . . . . . . . .

2.30 Species mole fraction as a function of temperature for $0.25 \%$ n-dodecane pyrolysis in the atmospheric pressure tube reactor at $40 \mathrm{~ms}$ residence time. Symbols are the experimental data with error bar indicating the uncertainty of the data. Lines are the model prediction using four different chemistry mechanisms. . . . . . . . . . . . . . .

2.31 Species mole fraction as a function of temperature for $0.25 \% \mathrm{n}$-Dodecane pyrolysis in the atmospheric pressure tube reactor at $50 \mathrm{~ms}$ residence time. Symbols are the experimental data with error bar indicating the uncertainty of the data. Lines are the model prediction using four different chemistry mechanisms. . . . . . . . . . . . . .

2.32 Species mole fraction as a function of temperature for $0.25 \%$ n-dodecane pyrolysis in the atmospheric pressure tube reactor at $50 \mathrm{~ms}$ residence time. Symbols are the experimental data with error bar indicating the uncertainty of the data. Lines are the model prediction using four different chemistry mechanisms. . . . . . . . . . . . . . .

2.33 Species mole fraction as a function of temperature for $0.25 \% \mathrm{n}$-Dodecane pyrolysis in the atmospheric pressure tube reactor at $60 \mathrm{~ms}$ residence time. Symbols are the experimental data with error bar indicating the uncertainty of the data. Lines are the model prediction using four different chemistry mechanisms. . . . . . . . . . . . . . .

2.34 Species mole fraction as a function of temperature for $0.25 \%$ n-dodecane pyrolysis in the atmospheric pressure tube reactor at $60 \mathrm{~ms}$ residence time. Symbols are the experimental data with error bar indicating the uncertainty of the data. Lines are the model prediction using four different chemistry mechanisms. . . . . . . . . . . . . .

2.35 Reaction rate constant fit for the first order lumped reaction for JP-8 pyrolysis. Symbols show the experimental data from the UVa tube reactor. . . . . . . . . . . . . . . . .

2.36 Comparison of model predictions of product species mole fraction in JP-8 pyrolysis data. Symbols are UVa tube reactor experiments, Solid blue line is the developed one-step model, dashed red line is Dagaut and Cathonnet model [80], dash-dot green line is Wang [81]. Tube reactor operating condition: $0.25 \% \mathrm{JP}-8$ fuel diluted in nitrogen, $\mathrm{p}=1$ atm, $\mathrm{T}=1000$ K. . . . . . . . . . . . . . . . 
2.37 Comparison of model predictions of product species mole fraction in JP-8 pyrolysis data. Symbols are UVa tube reactor experiments, Solid blue line is the developed one-step model, dashed red line is Dagaut and Cathonnet model [80], dash-dot green line is Wang [81]. Tube reactor operating condition: $0.25 \% \mathrm{JP}-8$ fuel diluted in nitrogen, $\mathrm{p}=1$ atm, $\mathrm{T}=1100$ K. . . . . . . . . . . . . . . .

3.1 Largest sensitivities of n-dodecane decomposition to reaction rates for n-dodecane pyrolysis in a flow reactor with atmospheric pressure, temperature of $1100 \mathrm{~K}$, and residence time of $20 \mathrm{~ms}$ using JetSurF model.

3.2 Normalized local sensitivity coefficients of ethylene production for the most important reactions in n-dodecane pyrolysis in a flow reactor with atmospheric pressure, temperature of $1100 \mathrm{~K}$, and residence time of 20 ms using JetSurF model. . . . . . . . . . . . . . . . . . . . . . . . . 71

3.3 Reaction path flux analysis for $\mathrm{n}$-dodecane pyrolysis at $\mathrm{p}=1 \mathrm{~atm}, \mathrm{~T}=1100$ K and 4 ns. . . . . . . . . . . . . . . . . . . 72

3.4 Reaction path flux analysis for $\mathrm{n}$-dodecane pyrolysis at $\mathrm{p}=1 \mathrm{~atm}, \mathrm{~T}=1100$

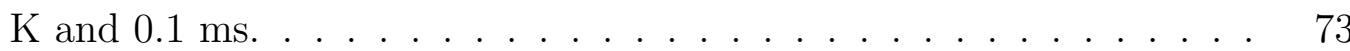

3.5 Reaction path flux analysis for $\mathrm{n}$-dodecane pyrolysis at $\mathrm{p}=1 \mathrm{~atm}, \mathrm{~T}=1100$ $\mathrm{K}$ and $1 \mathrm{~ms}$. . . . . . . . . . . . . . . . . . 74

3.6 Reaction path flux analysis for $n$-dodecane pyrolysis at $\mathrm{p}=1 \mathrm{~atm}, \mathrm{~T}=1100$ $\mathrm{K}$ and $10 \mathrm{~ms}$. . . . . . . . . . . . . . . . . 76

3.7 A comparison of sample distributions for two sampling methods in a two-dimension parameter space with $\mathrm{N}=1024$ number of samples from [86]. (a) Random sampling and (b) quasi-random Sobol' sequence sample. 77

3.8 The reaction rate coefficient of $\mathrm{CH} 4+\mathrm{H} \rightarrow \mathrm{CH} 3+\mathrm{H} 2$ taken from literature (Baulch et al. [87]) . The solid line is the recommended reaction rate expression and the dashed lines indicate the upper and lower bounds according to the suggested uncertainty factor of 2 . . . .

3.9 Species mole fraction profile as a function of time for n-dodecane pyrolysis in a $1100 \mathrm{~K}$ constant temperature and atmospheric pressure tube reactor. Symbols are UVa reactor experimental data with the error bars indicate uncertainty of the data and lines are model predictions with nominal rates. The shaded area indicates the $2 \sigma$ model prediction uncertainty. . . . . . . . . . . . . . . .

3.10 Species mole fraction profile as a function of time for n-dodecane pyrolysis in a $1100 \mathrm{~K}$ constant temperature and atmospheric pressure tube reactor. Symbols are UVa reactor experimental data with the error bars indicate uncertainty of the data and lines are model predictions with nominal rates. The shaded area indicates the $2 \sigma$ model prediction uncertainty. . . . . . . . . . . . . . . . 
3.11 Species mole fraction profile as a function of time for n-dodecane pyrolysis in a $1100 \mathrm{~K}$ constant temperature and atmospheric pressure tube reactor. Symbols are UVa reactor experimental data with the error bars indicate uncertainty of the data and lines are model predictions with nominal rates. The shaded area indicates the $2 \sigma$ model prediction uncertainty. . . . . . . . . . . . . . . .

3.12 Species mole fraction profile as a function of time for n-dodecane pyrolysis in a $1100 \mathrm{~K}$ constant temperature and atmospheric pressure tube reactor. Symbols are UVa reactor experimental data with the error bars indicate uncertainty of the data and lines are model predictions with nominal rates. The shaded area indicates the $2 \sigma$ model prediction uncertainty. . . . . . . . . . . . . . . .

3.13 Species mole fraction profile as a function of time for n-dodecane pyrolysis in a $1100 \mathrm{~K}$ constant temperature and atmospheric pressure tube reactor. Symbols are UVa reactor experimental data with the error bars indicate uncertainty of the data and lines are model predictions with nominal rates. The shaded area indicates the $2 \sigma$ model prediction uncertainty. . . . . . . . . . . . . . . .

3.14 Sensitivity of acetylene formation to reaction rates for $\mathrm{n}$-dodecane pyrolysis at 1 atm, $1100 \mathrm{~K}$, and $20 \mathrm{~ms}$ using Wang et al. model. The brute-force sensitivity coefficient is calculated by $\left\{d\left[\mathrm{C}_{2} \mathrm{H}_{2}\right] / d k_{i}\right\} \times$ $\left\{k_{i} /\left[\mathrm{C}_{2} \mathrm{H}_{2}\right]\right\}$, where $k_{i}$ is the $i$ th reaction rate constant. . . . . . .

3.15 Sensitivity of acetylene formation to reaction rates for n-dodecane pyrolysis at 1 atm, $1100 \mathrm{~K}$, and $20 \mathrm{~ms}$ using Banerjee et al. model. The brute-force sensitivity coefficient is calculated by $\left\{d\left[\mathrm{C}_{2} \mathrm{H}_{2}\right] / d k_{i}\right\} \times$ $\left\{k_{i} /\left[\mathrm{C}_{2} \mathrm{H}_{2}\right]\right\}$, where $k_{i}$ is the $i$ th reaction rate constant. . . . . . .

3.16 Sensitivity of acetylene formation to reaction rates for n-dodecane pyrolysis at 1 atm, $1100 \mathrm{~K}$, and $20 \mathrm{~ms}$ using Ranzi et al. model. The brute-force sensitivity coefficient is calculated by $\left\{d\left[\mathrm{C}_{2} \mathrm{H}_{2}\right] / d k_{i}\right\} \times$ $\left\{k_{i} /\left[\mathrm{C}_{2} \mathrm{H}_{2}\right]\right\}$, where $k_{i}$ is the $i$ th reaction rate constant. . . . . . .

3.17 Sensitivity of acetylene formation to reaction rates for $\mathrm{n}$-dodecane pyrolysis at $1 \mathrm{~atm}, 1100 \mathrm{~K}$, and $20 \mathrm{~ms}$ using Mze-Ahmed et al. model. The brute-force sensitivity coefficient is calculated by $\left\{d\left[\mathrm{C}_{2} \mathrm{H}_{2}\right] / d k_{i}\right\} \times$ $\left\{k_{i} /\left[\mathrm{C}_{2} \mathrm{H}_{2}\right]\right\}$, where $k_{i}$ is the $i$ th reaction rate constant. . . . . . .

3.18 Comparison of the recommended reaction rate coefficient of $\mathrm{C} 2 \mathrm{H} 4+\mathrm{CH} 3$ $\rightarrow \mathrm{C} 2 \mathrm{H} 3+\mathrm{CH} 4$ between four kinetic models. . . . . . . . . . . . . .

3.19 Size of different mechanisms for hydrocarbon fuels, with the number of species and reactions included in the mechanisms and the approximate year when the mechanisms were developed (figure from [90]). . . . . .

3.20 The $L^{2}$-norm error for the prediction of $n$-dodecane pyrolysis vs. the number of species in the reduced order skeletal model. . . . . . . . . .

3.21 Comparison of the predicted species mole fraction profiles between the detailed model and skeletal models with 50, 40, and 36 species. The conditions are for $0.25 \% \mathrm{n}$-dodecane pyrolysis in the atmospheric pressure tube reactor at $1050 \mathrm{~K}$. . . . . . . . . . . . . . . . 
3.22 Comparison of the predicted species mole fraction profiles between the detailed model and skeletal models with 50, 40, and 36 species. The conditions are for $0.25 \% \mathrm{n}$-dodecane pyrolysis in the atmospheric pressure tube reactor at $1050 \mathrm{~K}$. . . . . . . . . . . . . . .

3.23 Normalized local sensitivity coefficients of $\mathrm{C}_{2} \mathrm{H}_{6}$ pyrolysis at $\mathrm{p}=1 \mathrm{~atm}$ and $\mathrm{T}=1100 \mathrm{~K}$ evaluated at $50 \mathrm{~ms}$. The sensitivity is defined as $\left\{d\left[C_{2} H_{6}\right] / d k_{i}\right\} \times\left\{k_{i} /\left[C_{2} H_{6}\right]\right\}$, where $k_{i}$ is the $i$ th reaction rate constant. . . . . . . . . . . . . . . . .

3.24 Reaction path flux analysis for $\mathrm{C}_{2} \mathrm{H}_{6}$ pyrolysis at $\mathrm{p}=1 \mathrm{~atm}, \mathrm{~T}=1100 \mathrm{~K}$ and 0.2 ns. . . . . . . . . . . . . . . . . . . . . . . . . . . . . 97

3.25 Reaction path flux analysis for $\mathrm{C}_{2} \mathrm{H}_{6}$ pyrolysis at $\mathrm{p}=1 \mathrm{~atm}, \mathrm{~T}=1100 \mathrm{~K}$ and $0.1 \mathrm{~ms} . \ldots \ldots \ldots \ldots \ldots$

3.26 Reaction path flux analysis for $\mathrm{C}_{2} \mathrm{H}_{6}$ pyrolysis at $\mathrm{p}=1$ atm, $\mathrm{T}=1100 \mathrm{~K}$ and $50 \mathrm{~ms}$. . . . . . . . . . . . . . . . . . . 98

3.27 Reaction path flux analysis for $\mathrm{C}_{2} \mathrm{H}_{6}$ pyrolysis at $\mathrm{p}=1 \mathrm{~atm}, \mathrm{~T}=1100 \mathrm{~K}$ and $100 \mathrm{~ms} . \ldots \ldots \ldots$. . . . . . . . . . . . . . . 98

3.28 Mole fraction as a function of temperature . . . . . . . . . . . . . . 99

3.29 Mole fraction as a function of temperature . . . . . . . . . . . . . . 99

3.30 Mole fraction as a function of temperature . . . . . . . . . . . . 100

3.31 Mole fraction as a function of temperature . . . . . . . . . . . . 100

3.32 Mole fraction as a function of temperature . . . . . . . . . . . . 101

3.33 Mole fraction as a function of temperature . . . . . . . . . . . . 101

3.34 Mole fraction as a function of temperature . . . . . . . . . . . . . 102

3.35 Mole fraction as a function of temperature . . . . . . . . . . . . . 102

3.36 Mole fraction as a function of temperature . . . . . . . . . . . . 103

3.37 Normalized local sensitivity coefficients of $\mathrm{C}_{4} \mathrm{H}_{10}$ pyrolysis at $\mathrm{p}=1 \mathrm{~atm}$ and $\mathrm{T}=1100 \mathrm{~K}$ evaluated at $50 \mathrm{~ms}$. The sensitivity is defined as $\left\{d\left[C_{4} H_{10}\right] / d k_{i}\right\} \times\left\{k_{i} /\left[C_{4} H_{10}\right]\right\}$, where $k_{i}$ is the $i$ th reaction rate constant. 105

3.38 Reaction path flux analysis for $\mathrm{C}_{4} \mathrm{H}_{10}$ pyrolysis at $\mathrm{p}=1$ atm, $\mathrm{T}=1100$ $\mathrm{K}$ and 4 ns. . . . . . . . . . . . . . . . . 106

3.39 Reaction path flux analysis for $\mathrm{C}_{4} \mathrm{H}_{10}$ pyrolysis at $\mathrm{p}=1$ atm, $\mathrm{T}=1100$

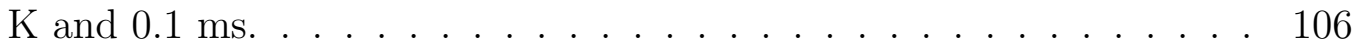

3.40 Reaction path flux analysis for $\mathrm{C}_{4} \mathrm{H}_{10}$ pyrolysis at $\mathrm{p}=1$ atm, $\mathrm{T}=1100$ $\mathrm{K}$ and $10 \mathrm{~ms}$. . . . . . . . . . . . . . . . . . 107

3.41 Mole fraction as a function of temperature . . . . . . . . . . . 107

3.42 Mole fraction as a function of temperature . . . . . . . . . . . . . . 108

3.43 Mole fraction as a function of temperature . . . . . . . . . . . . 108

3.44 Mole fraction as a function of temperature . . . . . . . . . . . . . 109

3.45 Mole fraction as a function of temperature . . . . . . . . . . . . . . 109

3.46 Mole fraction as a function of temperature . . . . . . . . . . . . . . 110

3.47 Mole fraction as a function of temperature . . . . . . . . . . . . . . . 110

3.48 Mole fraction as a function of temperature . . . . . . . . . . . . . 111

3.49 Mole fraction as a function of temperature . . . . . . . . . . . . . 111 
4.1 Schematic of a parabolic flow reactor with species profiles as a function of the axial and radial coordinate. . . . . . . . . . . . . . . . . . 114

4.2 Wedge geometry used for axi-symmetric modeling with OpenFOAM solver. . . . . . . . . . . . . . . . . . 116

4.3 The two-dimensional axisymmetric solution contour for temperature $(\mathrm{K})$. . . . . . . . . . . . . . . . .

4.4 The two-dimensional axisymmetric solution contour for n-C12H26 mole fraction. . . . . . . . . . . . . . . . . . . . 119

4.5 The two-dimensional axisymmetric solution contour for $\mathrm{H} 2$ mole fraction. 119

4.6 The two-dimensional axisymmetric solution contour for $\mathrm{CH} 4$ mole fraction. . . . . . . . . . . . . . . . . . . . . 119

4.7 The two-dimensional axisymmetric solution contour for $\mathrm{C} 2 \mathrm{H} 4$ mole fraction. . . . . . . . . . . . . . . . . . . 120

4.8 The two-dimensional axisymmetric solution contour for $\mathrm{C} 4 \mathrm{H} 6$ mole fraction. . . . . . . . . . . . . . . . . . . . 120

4.9 The two-dimensional axisymmetric solution contour for C7H14 mole fraction. . . . . . . . . . . . . . . . . 120

4.10 The two-dimensional axisymmetric solution contour for C8H16 mole fraction. . . . . . . . . . . . . . . . .

4.11 The two-dimensional axisymmetric solution contour for $\mathrm{C} 10 \mathrm{H} 20$ mole fraction. . . . . . . . . . . . . . . .

4.12 The two-dimensional axisymmetric solution contour for $\mathrm{C} 11 \mathrm{H} 22$ mole fraction. . . . . . . . . . . . . . . .

4.13 Species normalized mole fraction profile across the radial coordinate at $\mathrm{x}=1 \mathrm{~cm}$ from the reactor inflow.

4.14 Species normalized mole fraction profile across the radial coordinate at $\mathrm{x}=5 \mathrm{~cm}$ from the reactor inflow. . . . . . . . . . . . . . . . . . 125

4.15 Species normalized mole fraction profile across the radial coordinate at $\mathrm{x}=8 \mathrm{~cm}$ from the reactor inflow.

4.16 Species normalized mole fraction profile across the radial coordinate at $\mathrm{x}=15 \mathrm{~cm}$ from the reactor inflow.

4.17 Species normalized mole fraction profile across the radial coordinate at $\mathrm{x}=40 \mathrm{~cm}$ from the reactor inflow.

4.18 Comparison of temperature profiles for the two-dimensional axisymmetric n-dodecane pyrolysis. Solid line is the solution along the centerline axis of the tube and dashed line is the solution at the wall of the reactor

4.19 Comparison of species mole fraction profiles for the two-dimensional axisymmetric n-dodecane pyrolysis. Solid line is the solution along the center-line axis of the tube and dashed line is the solution at the wall of the reactor. . . . . . . . . . . . . . . .

4.20 Comparison of species mole fraction profiles for the two-dimensional axisymmetric n-dodecane pyrolysis. Solid line is the solution along the center-line axis of the tube and dashed line is the solution at the wall of the reactor 
4.21 Comparison of species mole fraction profiles for the two-dimensional axisymmetric n-dodecane pyrolysis. Solid line is the solution along the center-line axis of the tube and dashed line is the solution at the wall of the reactor. . . . . . . . . . . . . . . . . .

4.22 Temperature profiles along the tube reactor for n-dodecane pyrolysis. Solid line: 2D axisymmetric solution averaged over the cross-section of the tube reactor. Dashed line: 1D constant temperature solution. Dashed dotted line: 0D constant enthalpy (adiabatic) solution. . . . .

4.23 Species mole fraction profiles along the tube reactor for n-dodecane pyrolysis. Solid line: 2D axisymmetric solution averaged over the crosssection of the tube reactor. Dashed line: 1D constant temperature solution. Dashed dotted line: 0D constant enthalpy (adiabatic) solution.133

4.24 Species mole fraction profiles along the tube reactor for $\mathrm{n}$-dodecane pyrolysis. Solid line: 2D axisymmetric solution averaged over the crosssection of the tube reactor. Dashed line: 1D constant temperature solution. . . . . . . . . . . . . . . . .

4.25 Species mole fraction profiles along the tube reactor for $\mathrm{n}$-dodecane pyrolysis. Solid line: 2D axisymmetric solution averaged over the crosssection of the tube reactor. Dashed line: 1D constant temperature solution. . . . . . . . . . . . . . . . . .

4.26 Species mole fraction profiles along the tube reactor for $\mathrm{n}$-dodecane pyrolysis. Solid line: 2D axisymmetric solution averaged over the crosssection of the tube reactor. Dashed line: 1D constant temperature solution. . . . . . . . . . . . . . . . . .

5.1 Time scales in chemically reacting flows [99]. . . . . . . . . . . . . . . 140

5.2 UVaSCF flowpath with cavity flame-holder [102]. . . . . . . . . . . . 142

5.3 Schematic of the UVa supersonic combustion facility [106]. . . . . . . 146

5.4 Dimensions of UVa supersonic combustion facility, reactor configuration E, with the Mach 2 nozzle, side view (top) and top view (bottom) $[107] \ldots \ldots$. . . . . . . . . . . . . . . . . . . . . . . . . . . . . 147

5.5 Cavity residence time distribution [109]. . . . . . . . . . . . . . . . 149

5.6 Schematic of the stochastic mixing of the PaSR model, represented by $\mathrm{N}$ particles with the probability distribution of temperature and mass fraction changing as a function time. . . . . . . . . . . . . . . 151

5.7 Injector and combustion schematic with PaSR modeling. . . . . . . . 152

5.8 PaSR temperature profile for different reactor initial temperatures. . . 153

5.9 PaSR temperature profile for different reactor residence times. . . . . 154

5.10 PaSR temperature profile for different reactor mixing times. . . . . . 155

5.11 Temperature profile of PaSR for various ratios of the cavity flow to the main flow. . . . . . . . . . . . . . . . . . . . 156

5.12 Ignition delay time calculation for ramjet mode combustor at different equivalence ratios of ethylene-air combustion. . . . . . . . . . . . . 157

5.13 Schematic of the mixing cavity flow with the main flow and ignition in a closed homogeneous reactor. . . . . . . . . . . . . . . 158 
5.14 Ignition delay time as a function of inflow temperature for the ramjet mode ethylene-air combustion for different mixing percentages of cavity flow to the main flow. . . . . . . . . . . . . . . . . . . 159

5.15 Schematic of the mixing cavity flow with the main flow in a partially stirred reactor. The cavity flow is modeled by a perfectly stirred reactor. 160

5.16 Comparison of ignition delay time between PaSR model and closed homogeneous reactor model for the ramjet mode ethylene-air combustion. The PaSR simulation consist of mixing $2 \%$ cavity flow (PSR mixture) with $98 \%$ unreacted main flow. . . . . . . . . . . . . . . . .

5.17 Model reduction error for a stoichiometric mixture of ethylene-air combustion. . . . . . . . . . . . . . . . . 163

5.18 Ignition delay time as a function of initial temperature for ethylene-air stoichiometric mixture under atmospheric pressure. . . . . . . . . . . 164

5.19 Comparison of ethylene ignition delay time of detailed and reduced model as a function of initial temperature for different reacting conditions. Pressure $1 \mathrm{~atm}$ and $10 \mathrm{~atm}$, and equivalence ratio of $0.5,1$, and 2 are shown. . . . . . . . . . . . . . . . . 165

6.1 Schematic of the three one-dimensional flow cases with change in only one physical condition. . . . . . . . . . . . . . . . . . . . . . 169

6.2 Control volume of heating a variable area flow. . . . . . . . . . . . . . 170

6.3 Normalized wall pressures along the dual-mode supersonic combustor with various fuel injection configurations [107]. . . . . . . . . . . . . 172

6.4 Area ratio curve vs. ethylene-air equivalence ratio at thermally choked condition. Solid line is using a simple model without chemical dissociation and constant $c_{p}$, dashed line is based on chemical equilibrium calculation (adiabatic) with full detailed chemistry and real gas thermodynamics. . . . . . . . . . . . . . . . . . . . . 179 


\section{List of Tables}

3.1 Reactions selected for uncertainty propagation investigation for n-dodecane pyrolysis. . . . . . . . . . . . . . . . . . 80

3.2 Reactions selected for uncertainty propagation investigation for ethane pyrolysis. . . . . . . . . . . . . . . . . . 103

3.3 Reactions selected for uncertainty propagation investigation for n-butane pyrolysis. . . . . . . . . . . . . . . . . . . . . . . . 104

5.1 Experimental conditions for the scramjet combustor. . . . . . . . . . 148

5.2 Flow conditions for the ramjet combustor. . . . . . . . . . . . . . . . 148 


\section{Chapter 1}

\section{Introduction}

\subsection{Hypersonic Airbreathing Propulsion}

Historically, rocket-based propulsion systems have been used for hypersonic vehicles. Hypersonic flight is commonly referred to flight speeds of Mach 5 and above (i.e., flying faster than 5 times the local speed of sound). These high flight speeds are reached for applications such as planetary re-entry and space launch systems. Rocket-based propulsion systems need to carry oxidizer along with the fuel. Therefore, rocket boosted vehicles are heavy and have a very low specific impulse, $I_{s p}$, which is a metric use to quantify propulsion efficiency. It is defined as

$$
I_{s p}=\frac{\mathcal{T}}{\dot{W}}
$$

where $\mathcal{T}$ is the produced thrust and $\dot{W}$ is the weight of propellant consumed per unit time. Higher specific impulse means that the propulsion system performs more efficiently compared to propulsion system with lower specific impulse [1].

Figure 1.1 shows the specific impulse for several propulsion systems. Air-breathing propulsion systems (i.e., turbojets, ramjets, and scramjets) have orders of magnitude higher specific impulse than rocket boosters. This is due to the fact that a rocket 
powered vehicle must carry both fuel and oxidizer, whereas an air-breathing vehicle uses oxygen from the atmosphere and only needs to carry fuel. The elimination of oxidizer from the propulsion system reduces the weight of the vehicle significantly. Therefore, hypersonic airbreathing propulsion systems offer a new better system for long range flights and routine access to space missions within the atmosphere.

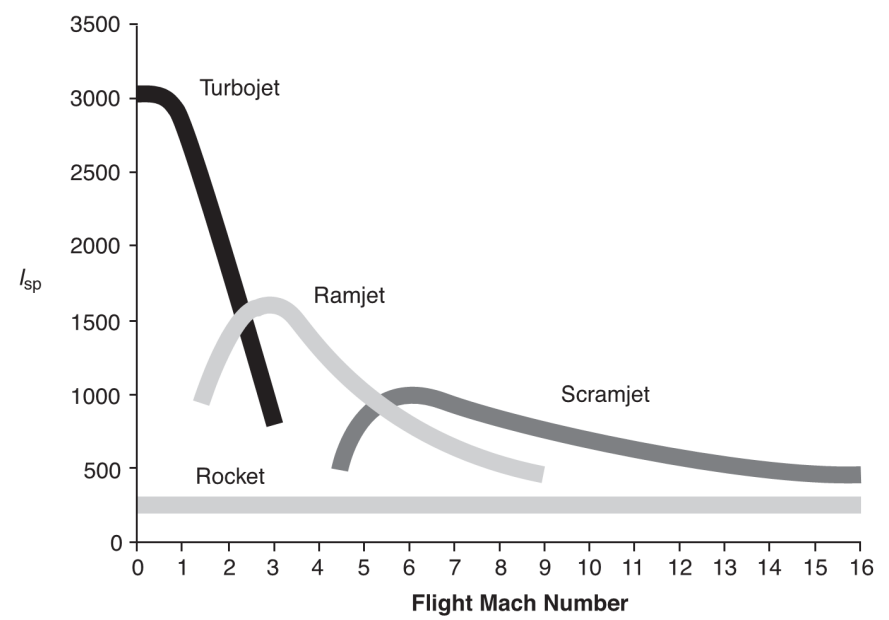

Figure 1.1: Performance (specific impulse) of air-breathing systems versus rockets as a function of flight Mach number [2].

Although hypersonic airbreathing propulsion systems have significant advantages and benefits compared to rocket propelled system, still an operational system has not been developed, due to difficulties associated with the design and analysis of hypersonic propulsion systems. There is a significant amount of research and development focus on hypersonic airbreathing propulsion in order to be able to develop the future reusable launch vehicles for efficient space access. At hypersonic speeds, aerodynamics and thermodynamic effects play a more important role in airbreathing system compared to slower speeds. At these flight conditions, significant temperature and pressure rise impose a constrain on the engine components. Also the air inflow condition with low residence time introduces a great challenge for the mixing and complete ignition of the fuel and air inside the combustor. Understanding each one of the problems associated with hypersonic airbreathing propulsion systems, necessitates 
accurate modeling capabilities in order to reduce the development cost and prevent any catastrophic failures. This guarantees that hypersonic vehicles will operate safely and reliably for long range and reusable operations.

Currently, none of the air-breathing engines operate individually from takeoff to hypersonic speeds. As shown in Figure 1.1, turbojets operate best from takeoff with subsonic speed up to flight speeds of Mach 3. Above flight speed of around Mach 3, compressors with rotating turbomachinery, are no longer needed to increase the pressure. As in a ramjet, the inflow air compression can be achieved by area variation in the inlet of the combustion chamber. This elimination of rotating parts enables the ramjet system to operate at higher maximum temperatures. Ramjets increase the pressure in the inlet section by slowing the supersonic incoming airflow to subsonic speeds through an accurate geometry change of the inlet and a series of shockwaves sitting in the inlet section. Then the subsonic compressed air is ducted into the combustion chamber, where it is mixed with fuel. Once the fuel/air mixture is burned in the combustor, the combustion product mixture is accelerated through a converging/diverging nozzle exiting the engine at supersonic speeds, producing thrust.

As flight speed further increases (speeds exceeding Mach 5), the losses associated with decelerating and compressing the supersonic inflow airstream to subsonic speeds become large, thus the supersonic combustion ramjet (i.e., scramjet) is preferred. The compression that results from decelerating the inflow from very high supersonic (above Mach 5) to subsonic velocities will begin to introduce a number of problems, including high static temperature and pressure exceeding the limits of engine components. Also the chemical dissociation due to the very high static temperature results in inefficient combustion, thereby significant energy loss. Moreover, the strong shockwaves associated with subsonic combustion leads to significant total pressure losses and thrust decrease [3]. In order to prevent these problems, it becomes more efficient to maintain the flow at supersonic speed in the combustor and throughout the engine, 
hence supersonic combustion ramjet, or scramjet.

As all the existing airbreathing engines have flight speed operation limitations, the combined cycle system is the feasible design that can safely handle the transition of a vehicle between different modes of propulsion. Combined cycle engine system enables efficient operability and performance over a wide range of flight conditions, from takeoff, to subsonic, to supersonic and hypersonic Mach numbers. Figure 1.2 shows an illustration of a combined cycle concept. A turbine-based engine accelerates the vehicle from takeoff (standing start) to supersonic speeds with Mach number around 3. Then the propulsion system switches from the low-speed turbojet to the high-speed ramjet/scramjet engine capable of efficiently operating from Mach 3 and reaching hypersonic Mach numbers of the order of 8-10 (for hydrocarbon fuels).

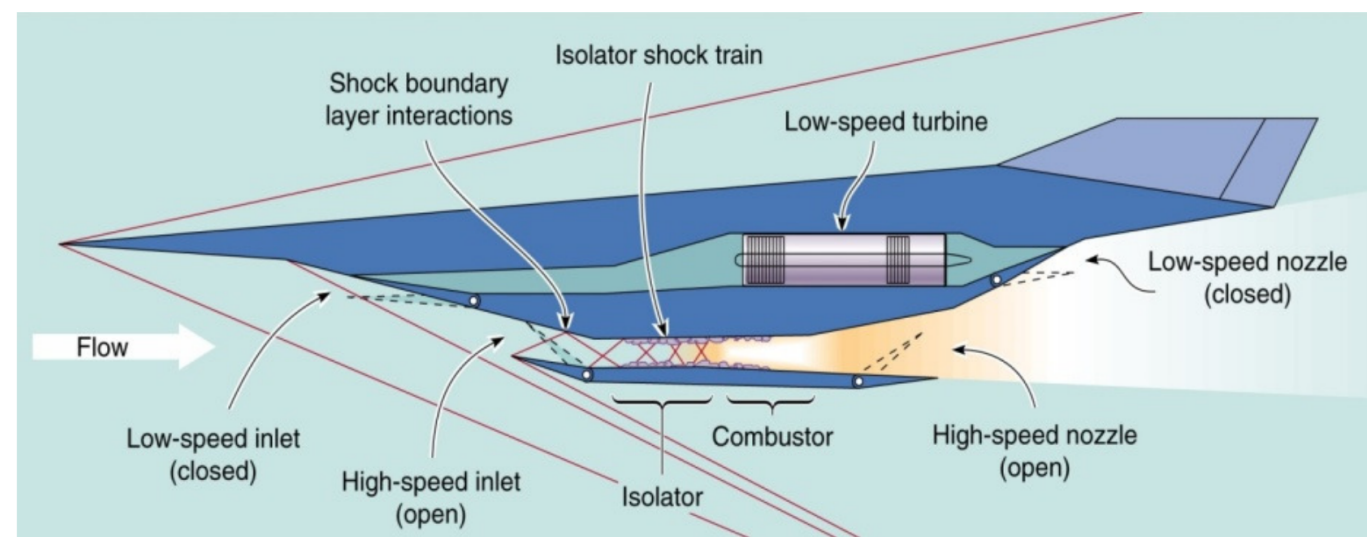

Figure 1.2: Turbine-based combined cycle hypersonic vehicle concept [4].

\subsection{Objectives of this Work}

The design and development of the hypersonic propulsion system consists of number of challenges. The objective of this study is to better understand and to develop suitable models for three outstanding challenges in the hypersonic airbreathing propulsion systems. The three main components of the hypersonic combustor that is investigated in this work are (see Figure 1.3): 
1. Endothermic pyrolysis of hydrocarbon fuels in the cooling channels.

2. The fuel-air mixing/reaction interaction in the cavity flame holding region of the combustor and its effect on the ignition time.

3. Thermal choking and operability limits in the diverging section of the dual-mode supersonic combustor.

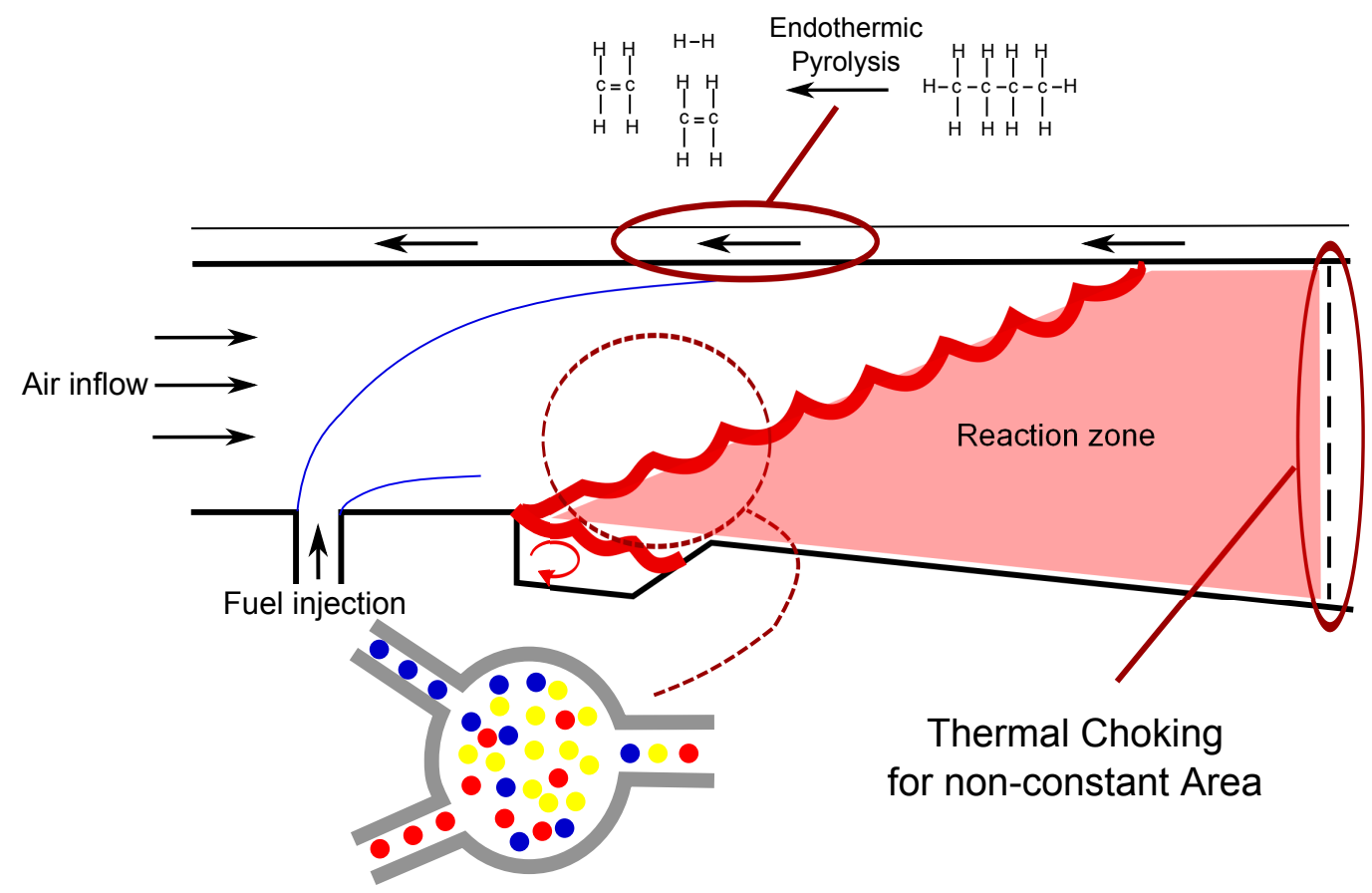

Partially stirred reactor model

Figure 1.3: Schematic of the hypersonic combustor. Three research focuses in this work are: (1) Engine cooling using fuel endothermic pyrolysis, (2) Chemical analysis of the fuel oxidation in the combustor, (3) Thermal choking and operability limits of hypersonic combustor.

Figure 1.3 shows a schematic of a supersonic combustor with the emphasis on the three main topics investigated in this work. The inflow air stream is mixed with the conventional aviation fuel injected upstream of the combustor. The strong turbulent mixing and chemical reaction interaction plays an important role in the combustor. Due to the very short residence time (of the order of milliseconds) of the air-fuel mixture in the engine, a cavity flame holder is present in the combustor to provide an 
increased residence time region for the fuel-air mixture and improve the flame stability in the combustor. The recirculation flow field of the cavity provides a radical pool source into the main flow field and enhances the flame holding and stability for a wider range of flight conditions.

The heat release from the combustion of the fuel-air mixture occurs in a diverging combustion area section. This diverging area helps to delay the thermal choking through heat release from the chemical reaction, therefore improving the operability limits of the engine. Otherwise, the amount of injected fuel is limited and higher fuel air ratios result in early thermal choking downstream of the combustion chamber.

Since this engine is operating at hypersonic flight conditions, both vehicle and the combustor engine components require active cooling. High temperature loads produced by the strong shockwaves and heat addition from the fuel combustion would exceed the structural temperature limits of any material. Therefore, active cooling process is required and the fuel on board is the most appropriate candidate. Using the fuel to cool the high temperature parts of the engine and vehicle, eliminates the need for a separate cooling system such as heat exchangers or any other cooling fluid, which adds to the weight of the vehicle. As shown in Figure 1.3, the fuel is passed through the cooling channels around the engine parts and absorbs the heat. As the temperature of the fuel (in this case hydrocarbon fuel) increases, after some temperature limit, the fuel starts to crack to smaller molecule hydrocarbons and hydrogen species. This chemical process is normally an endothermic process. The endothermic pyrolysis of hydrocarbon fuels absorbs extra heat from the cooling channel surface and increase the cooling capability.

The first objective of this work is on the endothermic pyrolysis of different hydrocarbon fuels in the cooling channel of hypersonic combustors. The detailed analysis of the modeling and comparison with the experimental data is performed. The chemical kinetic model uncertainties are investigated and compared against the experimental 
measurements uncertainty. Also the effect of the simplification of the cooling channel with a one-dimensional model is compared against a two-dimensional reacting flow simulation.

The second objective is on the fuel oxidation in the combustor with the focus on the turbulent mixing interactions with the chemical reaction and the effect on the combustion efficiency. Here the flame holding region is modeled by a simple Partially Stirred Reactor in order to include both the turbulent mixing time and flow convection time relevant to hypersonic flight conditions in the fuel-air combustion modeling.

The final objective is to develop a model to predict the thermal choking characteristics in the diverging area section of the scramjet combustor. The heat addition from the exothermic process of the combustion is calculated with detailed chemistry model and the area change is considered in order to predict the thermal choking at different subsonic to supersonic flight conditions.

\subsection{Overview}

The organization of the thesis is outlined below:

The first three chapters 2,3 , and 4 are on the analysis of endothermic fuel pyrolysis in the cooling channels of a hypersonic combustor. Four different fuels (ethane, n-butane, n-dodecane, and JP-8) are considered for the analysis. In chapter 2, endothermic fuel pyrolysis in cooling channel are investigated based on a tubular microflow reactor developed at UVa Combustion and Energy Lab. The studied tube reactor is a representation of real cooling channels at hypersonic combustors. For each fuel studied, several detailed mechanisms from literature are used with plug flow model and compared with experimental data. Also a simple one-step lumped reaction model for JP-8 pyrolysis is developed.

In chapter 3 , the uncertainty associated with the chemical kinetic model for the 
endothermic fuel pyrolysis is extensively analyzed. Sensitivity analysis and reaction pathway analysis are performed to identify the important reactions contributing to each fuel pyrolysis and governing the major species mole fractions at the corresponding conditions. Based on these findings, a computationally expensive Monte Carlo simulation with a a large sample size is performed in order to quantify the model uncertainty. The results are compared with the experimental data and measurements uncertainty. Furthermore, based on the analysis performed in this chapter, a reduced reaction mechanism is developed for n-dodecane pyrolysis to be used in multi-dimensional reacting flow simulation.

Chapter 4 investigates the two-dimensional effects of the laminar parabolic flow in the tubular reactor. A complete numerical axi-symmetric Navier-Stokes simulation including chemical reactions is performed in order to understand the radial gradients effect. The numerical calculation is based on the boundary conditions from the tube reactor experiment. The results from the two-dimensional numerical simulation are presented and compared with the one-dimensional plug flow simulation results.

Chapter 5 discusses the new approach to model the fuel oxidation in the dual-mode supersonic combustor. The cavity flame holder and the turbulent mixing and reaction interaction in the combustor are simulated by a computationally efficient implementation of partially stirred reactor model in connection with perfectly stirred reactor. The relevant time scales for hypersonic combustors are used for the simulations and the results are presented. A reduced reaction mechanism for ethylene combustion is developed and validated for a range of applicable hypersonic conditions.

In Chapter 6, a numerical model is developed to predict the thermal choking in a variable area (diverging nozzle) in dual-mode supersonic combustor. The model is used in order to find the operability limits of ethylene fuel combustion at the relevant conditions of the UVa supersonic combustion facility.

At the end of this thesis, the two reduced reaction mechanisms for n-dodecane 
pyrolysis and ethylene combustion are listed in the Appendices. 


\section{Chapter 2}

\section{Endothermic Fuel Pyrolysis in Cooling Channels}

\subsection{Background}

The hypersonic vehicles leading edges and engine components experience very high temperatures. Even the best materials could not withstand such large heat loads which requires the integration of an active cooling system. One possible option is to make use of the existing fuel line system and design it to not only transport the fuel but also serve as a heat sink. This would avoid the additional weight penalties associated with carrying a separate cooling fluid and heavy heat exchangers. The heat lost in this design is recovered by combustion of the heated fuel, which increases engine efficiency. Despite the promise of this regenerative cooling system, the balance of the necessary fuel flow rate with the required cooling rate adds additional complexity. For example, if the system requires larger fuel flow rates in order to meet the cooling requirement of the combustor, then the injected fuel may be too rich and decrease the efficiency. This requires a detailed analysis of fuels cooling capacity of the aviation fuel, and if needed, design a fuel bypass system to meet the vehicle cooling requirement for severe 
operating conditions.

Liquid Hydrocarbon fuels have high energy densities and are easier to operate and store compared to hydrogen fuel. This leads to smaller and lighter vehicles when hydrocarbons are used as the main fuel. Furthermore, the heat sink capability of decomposing hydrocarbon fuels are significantly enhanced because they are endothermic $^{1}$. The products of hydrocarbon decomposition are smaller molecules and are fuels with faster ignition times. Therefore, the decomposition not only enhances the fuel-cooling capability beyond the physical (sensible) capacity but also enhances the ignition delay time of the fuel for hypersonic combustion application.

The decomposition of the endothermic hydrocarbon fuels begins at temperatures around $900 \mathrm{~K}$. Above this temperature, the chemical heat sink rapidly increases, becoming comparable with the physical heat sink, essentially doubling the fuel heatsink capability $[5,6]$. Huang et al. [7] reported that the total heat sink improved by $20 \%$ to $40 \%$ for JP-7, JP- $8+100$, JP- 10 , and n-Octane for surface temperatures around $1000 \mathrm{~K}$.

Catalytic decomposition offers the additional ability to select the products of pyrolysis. The catalyst can be added as an additional component to fuel lines with the intention of controlling certain reaction pathways in order to decompose the fuel to the desired products. Lander and Nixon [5] studied the endothermic decomposition through the presence of a catalyst. Edwards [8] showed that product species like olefins (e.g. ethylene) are more desired compared to parafins (e.g. propane) to increase the endothermicity. Furthermore, the other advantage of using a catalyst is that it lowers the temperature at which the system operates by means of greater reaction rates at low temperatures [5].

The cooling capacity (heat sink) is defined as the enthalpy change of the fuel before entering the cooling channel and after exiting it. Figure 2.1 depicts a control

\footnotetext{
${ }^{1}$ Endothermic fuels absorb heat while undergoing a chemical decomposition.
} 
volume with fuel mass flow rate $\dot{m}$ absorbs the heat from channel surface. Using conservation of energy, the heat sink can be calculated by,

$$
Q_{\text {sink }}=\dot{m}\left(h_{\text {out }}-h_{\text {in }}\right)
$$

where $h_{\text {in }}$ and $h_{\text {out }}$ are the fuel enthalpies per unit mass at the inlet and exit, respectively.

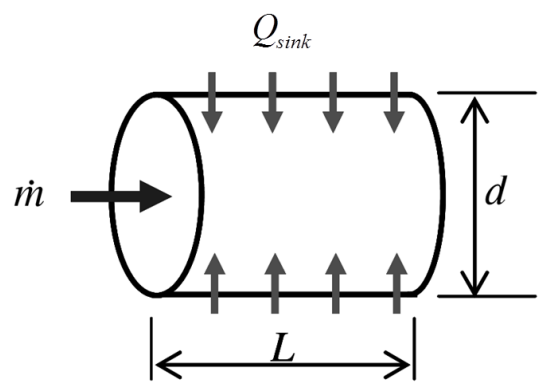

Figure 2.1: Heat transfer in the control volume of the cooling channel.

The total heat sink of fuel includes the sensible heating, $\Delta h_{\text {sens }}$, which is due to the increase in temperature, and endothermic heating, $\Delta h_{\text {endo }}$, which is due to the chemical reaction and composition change. This means that if the composition of the fuel does not change, the total heat sink will be equal to the sensible heat only. However, if the composition changes and pyrolysis occurs, there is an additional cooling due to the endothermic reactions which leads to higher cooling capacity.

To compute the sensible heat sink, the fuel composition remains constant and the enthalpy is only a function of temperature. Thus,

$$
\Delta h_{\text {sens }}=h\left(T_{\text {out }}\right)-h\left(T_{\text {in }}\right)
$$

The endothermic heat sink can be calculated by,

$$
\Delta h_{\text {endo }}=\frac{Q_{\text {sink }}}{\dot{m}}-\Delta h_{\text {sens }}
$$


The heat sink calculation for n-dodecane is shown in figure 2.2. As expected, the sensible heat increases linearly with temperature. However, the endothermic heat increases significantly for temperatures above $900 \mathrm{~K}$ which is the point where the fuel starts to crack to smaller species. At higher temperatures, the ratio of the endothermic heat over sensible heat increases which means enhanced cooling for higher temperatures.

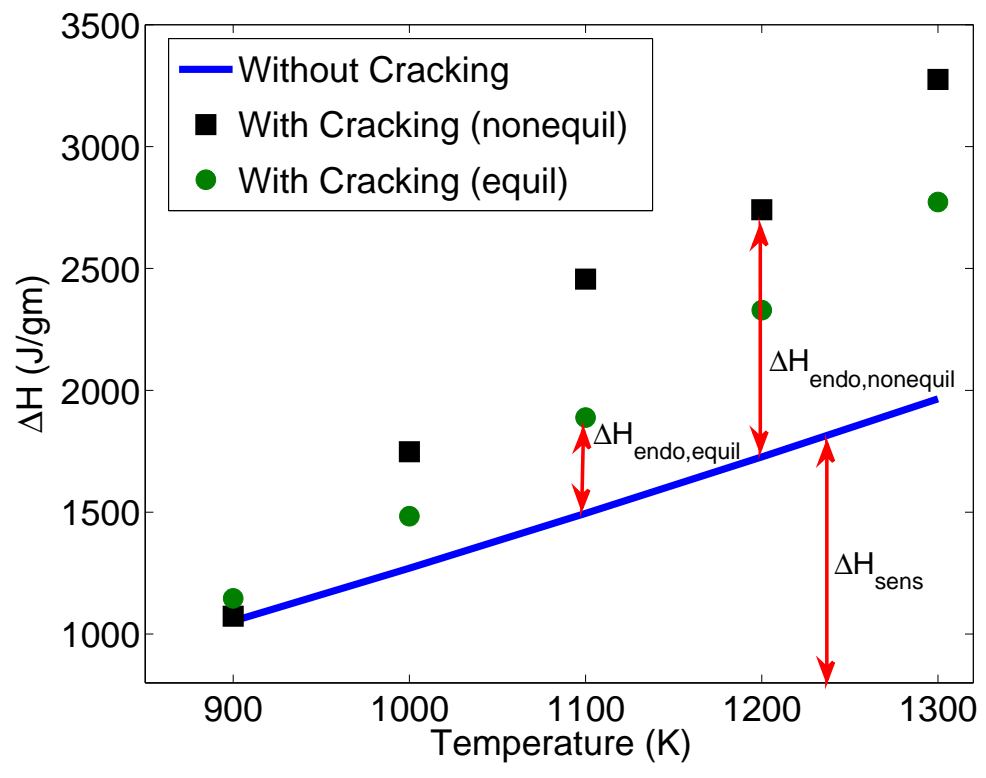

Figure 2.2: Heat sink for n-dodecane pyrolysis.

Also by comparing the endothermic decomposition of n-dodecane at two different states, it can be shown that the endothermicity is much higher for the nonequilibrium state $\left(\Delta \mathrm{H}_{\text {endo,nonequil }}\right)$ with finite residence time $\left(\tau_{\text {res }}=100 \mathrm{~ms}\right)$ versus the equilibrium state $\left(\Delta \mathrm{H}_{\text {endo,equil }}\right.$ at $\left.\tau_{\text {res }} \rightarrow \infty\right)$. The long residence time negative effect on the endothermicity is due to the formation of equilibrium products with lower enthalpy. These preliminary results were used in order to design the experimental setup with the operating conditions relevant to real endothermic pyrolysis in hypersonic combustor.

One negative aspect of using hydrocarbon fuels for cooling is the coke formation in the cooling channels. Coke is the solid deposits that result from the fuel decomposition 
and are undesirable in these systems. The coke deposition on the surface can degrade cooling performance by increasing both thermal resistance and fuel pressure drop. Eventually the coke deposition in the fuel line will lead to system failure by blocking the fuel cooling channels [7]. Therefore, it is important to study the production of soot precursors that lead to the formation of poly aromatic hydrocarbon (PAH) molecules and soot particles. Acetylene and benzene are among the species identified to be important in PAH formation in the literature $[9,10]$.

In this study, fuel decomposition in a tubular reactor is investigated. This tube reactor is designed to represent endothermic fuel pyrolysis in the cooling channel systems on the most fundamental level. It includes thermocouple controlled electrical heaters surrounding a quartz tube to provide an approximately uniform surface temperature, which is representative of the high surface temperature a cooling channel will be exposed to in real hypersonic engine.

Modeling of this tube reactor configuration is challenging depending on the level of accuracy required. The most computationally efficient model for tube reactor analysis is the zero-dimensional homogeneous-mixture model. The computational efficiency of this model enables the use of detailed chemical kinetic mechanisms and the exploration of various conditions experienced in the tube reactor. However, this model lacks the ability to capture any multi-dimensional effects which requires the use of computational fluid dynamics (CFD). In many past studies [11-13], to match the experimental data with the computational result from zero-dimensional model, some arbitrary time shifting has been performed. For example Held and Dryer [11] showed that the zero-dimensional simulation cannot model the nonideality of the mixing region upstream of the reactor. This nonideality only accelerates the reaction for a short time without effecting the concentration profiles. Therefore, shifting the experimental data to longer time provided a better comparison to computational result. In this work, there was no need for any time shifting of the experimental data. 
This is due to fact that the tube reactor used in this study was designed with such specifications that the mixing region is very small compared to the overall reactor residence time. Therefore, time shifting was not required. More details about the reactor are given in the following section.

\subsection{Experimental Methods}

\subsubsection{Introduction to Flow Reactors}

Flow reactors are among the experimental methodologies that are widely used to study the chemical kinetics of steady-state flow reacting systems. These reactors are usually tubular and the flow in the reactors are assumed to be plug flow in the idealized form. As illustrated in Figure 2.3, the idealized plug flow reactor assumes radially uniform profiles for temperature, velocity, and species concentration along the axial direction. This means in the idealized plug flow model, there is perfect mixing in the radial direction and no mixing in the axial direction. Despite axial gradients in species concentration, the plug flow reactor model assumes that the axial diffusion time scales are negligibly small in comparison to the advection and reaction time scales. As a consequence, spatial profiles of temperature and species mass fractions can be converted to temporal profiles under the relation between the axial location

and fluid residence time based on the flow velocity $(\mathrm{t}=\mathrm{x} / \mathrm{U})$. This equivalence of location/time duality of plug flow reactor constructs a more simplified mathematical formulation as an initial value problem similar to Eq. 2.4. 


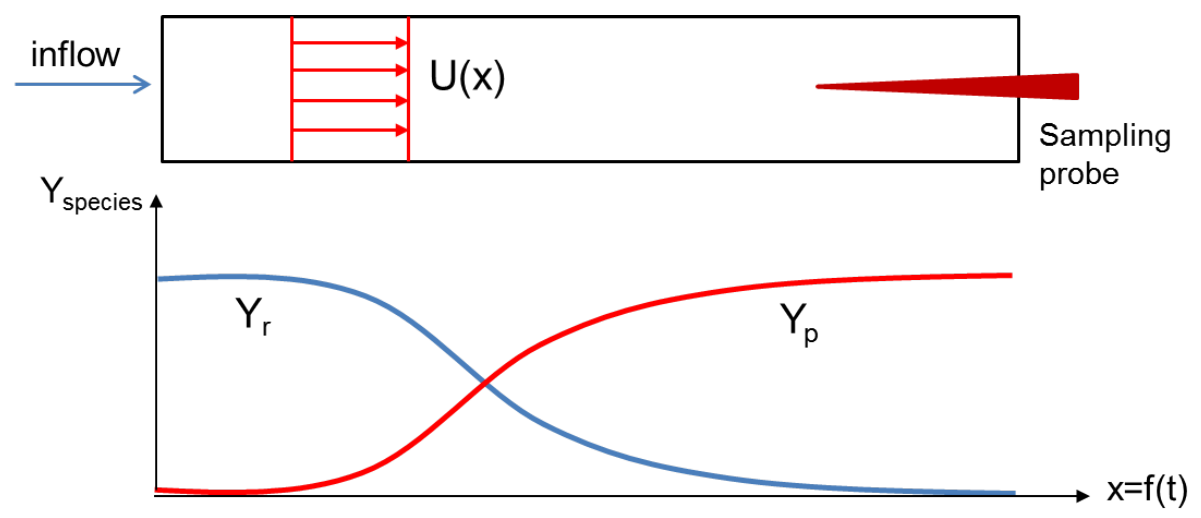

Figure 2.3: Schematic of an idealized plug flow reactor with species profiles as a function of the axial coordinate.

Generally, turbulent flow reactors are modeled by the idealized plug flow reactor assumption, where it has a top-hat velocity profile and the turbulence causes an intense radial mixing creating uniform transverse species profiles. Some examples of these turbulent flow reactors that are used for chemical kinetic studies include:

1) The Princeton Variable Pressure Flow Reactor (VPRF) [11]. In their reactor, nitrogen carrier gas is heated by electric resistance heaters and homogenously mixed with the injected fuel at the entrance to a movable conical shaped mixer/diffuser section that expands from a $2.5 \mathrm{~cm}$ diameter mixing region to a $10.2 \mathrm{~cm}$ internal diameter quartz test section. The reactor wall temperatures are maintained at the desired initial gas temperature by thermostated electrical resistance heaters. For species measurements, the reacting mixture is sampled at some axial position downstream of the mixer/diffuser using a hot-water-cooled, stainless steel sampling probe. They have recently used this reactor to investigate the kinetic behavior of major nalkanes from $\mathrm{C} 7$ to $\mathrm{C} 14$ at temperture ranges of $500 \mathrm{~K}$ to $1000 \mathrm{~K}$ and pressures of 8 atm and 12.5 atm with time histories of up to 3 s. [14, 15].

2) Pressurized Flow Reactor (PFR) facility at Drexel University [16]. The PFR is a turbulent flow reactor that consists of a $2.2 \mathrm{~cm}$ internal diameter, $40 \mathrm{~cm}$ long quartz tube within a pressure vessel. It can safely operate at temperatures up to 850 
$\mathrm{K}$ and withstand pressures up to 20 atm [17]. They have recently used this reactor to investigate the oxidation of $\mathrm{n}$-dodecane at low temperature range of $550 \mathrm{~K}$ to 830 $\mathrm{K}$, high pressure of $8 \mathrm{~atm}$, and lean equivalence ratio of 0.23 [16].

3) Stanford high-pressure flow reactor (HPFR) facility [18]. This reactor uses combustion products of an H2/Air flame to generate a hot vitiated flow in order to bring the initial flow temperature to the desired value. It is a vertically oriented quartz flow reactor that includes a converging-diverging duct attached to a constant $3 \mathrm{~cm}$ diameter, $30 \mathrm{~cm}$ long section. It can operating at pressures up to $25 \mathrm{~atm}$ and

temperatures up to $1300 \mathrm{~K}$. The two issues related to this reactor are the effect of vitiated flow in the chemical kinetic study and also the more complicated procedure in the modeling of the long diffuser mixing region. This reactor is recently used to investigate the pyrolysis and oxidation of n-dodecane at temperature ranges of 1000 $\mathrm{K}$ to $1300 \mathrm{~K}$, atmospheric pressure, and 1 to $40 \mathrm{~ms}$ residence times [19].

In the current study, UVa Micro-Flow Tube Reactor is used to study the pyrolysis of different n-alkane hydrocarbon fuels at temperature ranges of $1000 \mathrm{~K}$ to $1100 \mathrm{~K}$. The details of this reactor are explained in the following section.

\subsubsection{UVa Micro Flow Tube Reactor}

The schematic of the UVa Micro-Flow Tube Reactor (MFTR) is shown in Figure 2.4. A horizontally oriented quartz tube with a constant $4 \mathrm{~mm}$ inner diameter is inside a steel pressure chamber (hydro tested up to $100 \mathrm{~atm}$ ). The quartz tube reactor is heated to the target operating temperature using six independent electrical resistance heaters. The main flow (nitrogen carrier gas) is preheated to the target temperature in a $125 \mathrm{~cm}$ long helical section before mixing with the fuel under investigation introduced via two side tubes of $1 \mathrm{~mm}$ ID. A porous quartz frit placed immediately downstream of the junction between the side tubes and the main flow with a mixing volume of less than $0.2 \mathrm{~cm}^{3}$ facilitates rapid mixing, which has been verified by 
measurement of species mole fraction radially on a plane just downstream of the frit (prior to fusing the hot section). The hot test section (from the frit to the tube exit plane) is $37 \mathrm{~cm}$ long with six PID controllers connected via K-type thermocouples maintain the hot section at the target temperature. In supporting heater calibration experiments, the temperature inside and outside of the tube were measured under typical flow velocities and showed a difference of less than $5 \mathrm{~K}$ with inert gas flow. Endothermic pyrolysis of $2 \%$ ethane in nitrogen showed a small drop in temperature (less than $5 \mathrm{~K}$ ) compared to the inert case, while exothermic oxidation conditions showed a similarly slight increase in temperature. The variation in temperature due to endothermicity/exothermicity can be minimized by further dilution of the fuel. For example, n-dodecane was diluted in $99.75 \%$ nitrogen to minimize temperature departures from target values.

The experimental measurements of the UVa micro-flow tube reactor are conducted at Combustion and Energy Lab by my colleagues Shrestha and Simms. All the details of the experimental methodology and the reported data with uncertainties can be found in our recently submitted paper [20].

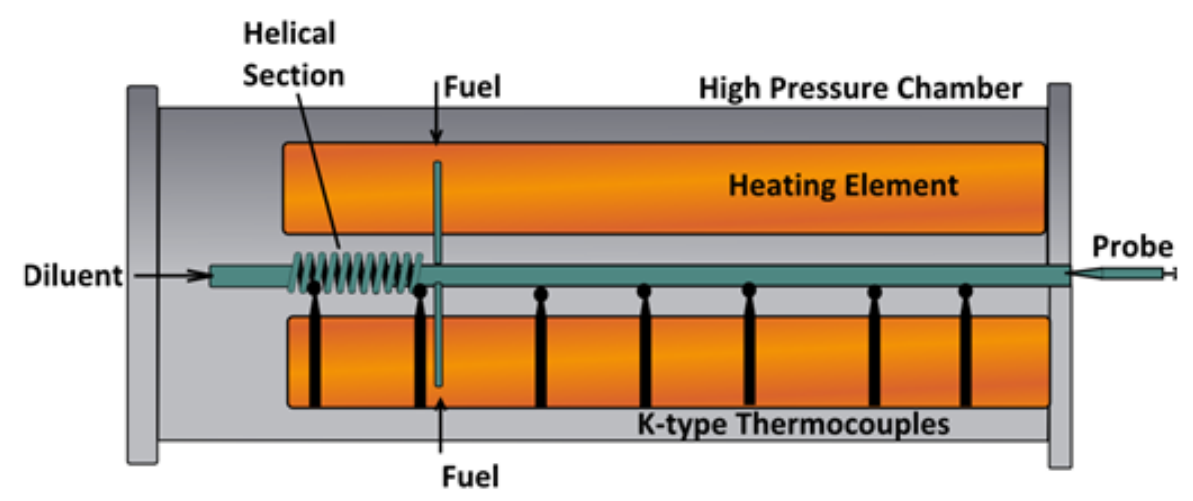

Figure 2.4: Schematic of UVa Micro-Flow Tube Reactor setup. Diluent is preheated in the helical section to the desired temperature. The fuel is added through the side tubes and mixes with the preheated gas and reacts along the tube with a near constant temperature variation. A quartz probe is used to transfer a sample to the gas analysis system. 


\subsection{Governing Equations}

\subsubsection{Zero-Dimensional Model Governing Equations}

The mass conservation equation for individual species in a zero-dimensional closed reactor (no mass crossing the boundary) are given by,

$$
\rho \frac{d Y_{k}}{d t}=\dot{\omega}_{k} W_{k}, \quad k=1, \ldots, K
$$

where $t$ is time, $Y_{k}$ is the mass fraction of species $k$ (there are total of $K$ species), $\dot{\omega}_{k}$ is the molar rate-of-production of the species $k$ by chemical reaction per unit volume, $W_{k}$ is the molecular weight of species $k$, and $\rho$ is the density of the gas mixture.

The corresponding energy conservation equation for the constant pressure reactor is given by,

$$
\rho c_{p} \frac{d T}{d t}+\sum_{k=1}^{K} h_{k} \dot{\omega}_{k} W_{k}=0
$$

where $T$ is temperature, $h_{k}$ is the enthalpy of species $k$, and $c_{p}$ is the mean specific heat of the mixture given by,

$$
c_{p}=\sum_{k=1}^{K} Y_{k} c_{p, k}
$$

The net chemical production rate $\dot{\omega}_{k}$ of each species is determined by all the chemical reactions involving that species. Each reaction proceeds according to the modified Arrhenius equation with temperature dependence, and the rate constant is given by:

$$
k=A T^{\beta} \exp \left(-\frac{E_{a}}{R T}\right)
$$

where $A$ is the pre-exponential factor, $\beta$ is the temperature exponent, $E_{a}$ is the activation energy, and $R$ is the universal gas constant. The first three parameters are 
specified for all the elementary reactions in every chemical kinetic mechanism.

Temporal integration of the above coupled equations are performed using Senkin Code [21] with thermochemical data from Chemkin [22]. Two types of initial and boundary conditions of the reactor were considered. In the first case, governing equations are integrated under the assumption of adiabatic boundary conditions, i.e. constant enthalpy such that under endothermic pyrolysis, the system temperature will decrease depending on the level of dilution and the endothermicity of the fuel. In the second case, constant temperature assumption is imposed with heat losses or gain through the reactor boundary. Clearly, at highly diluted conditions, both solutions approach each other.

\subsubsection{One-Dimensional Model Governing Equations}

To investigate the effects of axial transport in the presence of axial gradients of species and temperature, the following governing equations for a steady, isobaric, one-dimensional reacting flow are considered. The mass and energy equations are for the gas mixture, and the species equation is the mass transport for each of the individual species.

Mass continuity:

$$
\dot{M}=\rho u A
$$

where $\dot{M}$ denotes the mass flow rate (which is independent of $\mathrm{x}$ ), $u$ the velocity of the fluid mixture, $\rho$ the mass density of the mixture, and $A$ the cross-sectional area of the tube.

Species conservation:

$$
\dot{M} \frac{d Y_{k}}{d x}+\frac{d}{d x}\left(\rho A Y_{k} V_{k}\right)=A \dot{\omega}_{k} W_{k}, \quad k=1, \ldots, K
$$

where $x$ denotes the position, $Y_{k}$ the mass fraction of the $k^{t h}$ species, $W_{k}$ the molecular 
weight of the $k^{\text {th }}$ species, $\dot{\omega}_{k}$ the molar rate of production by chemical reaction of the $k^{\text {th }}$ species per unit volume, and $V_{k}$ the diffusion velocity of the $k^{\text {th }}$ species.

Energy conservation:

$$
\dot{M} \frac{d T}{d x}-\frac{1}{c_{p}} \frac{d}{d x}\left(\lambda A \frac{d T}{d x}\right)+\frac{A}{c_{p}} \sum_{k=1}^{K} \rho Y_{k} V_{k} c_{p, k} \frac{d T}{d x}+\frac{A}{c_{p}} \sum_{k=1}^{K} h_{k} \dot{\omega}_{k} W_{k}=0
$$

where $T$ denotes the temperature, $\lambda$ the thermal conductivity of the mixture, $c_{p}$ the constant-pressure heat capacity of the mixture, $c_{p, k}$ the constant pressure heat capacity of the $k^{t h}$ species, and $h_{k}$ the specific enthalpy of the $k^{t h}$ species.

The density of the mixture is given by the ideal gas law:

$$
\rho=\frac{p \bar{W}}{R T}
$$

where $R$ is the universal gas constant, $p$ is the pressure, and $\bar{W}$ is the mean molecular weight of the mixture.

The above equations are solved using Premix Code [23] by imposing the inflow boundary conditions and zero outflow gradients, with either constant temperature or constant enthalpy reactor conditions.

\subsection{Taylor Aris Dispersion}

Axial dispersion characterizes the spreading of the species in the axial direction due to both molecular diffusion and the radial velocity gradient. Taylor [24] and Aris [25] derived an equation for dispersion coefficient, $D_{\text {disp }}$, for a parabolic velocity profile in a laminar flow regime:

$$
D_{\text {disp }}=D_{i j}+\frac{V^{2} R^{2}}{48 D_{i j}}=D_{i j}\left(1+\frac{P e^{2}}{48}\right)
$$


where Peclet number is defined as,

$$
P e=\frac{V R}{D_{i j}}
$$

This coefficient is also called the effective diffusion coefficient. Molecular diffusion, $D_{i j}$, promotes the axial dispersion for low velocity, while it has an opposite effect at high velocity. There is an optimum relation between the molecular diffusion $D_{i j}$, velocity $V$, and tube radius $\mathrm{R}$ which makes the axial dispersion to be minimal. This optimum happens at $P e=\sqrt{48}$ where the ratio $D_{\text {disp }} / V R$ reaches its minimal value of 0.29 (see Figure 2.5).

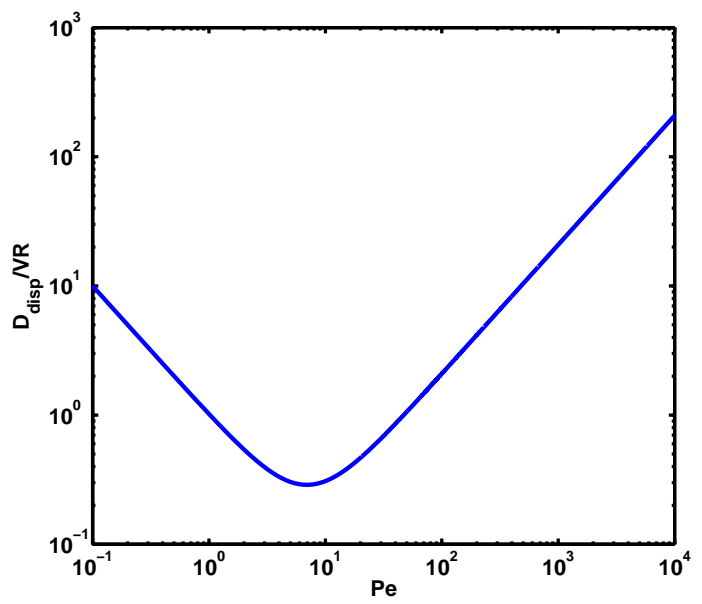

Figure 2.5: Nondimensionalized effective dispersion coefficient as a function of Peclet number (the minimum occurs at $P e=6.9$ )

A vessel dispersion number is defined by Levenspiel [26], that can be used to find quantitative deviation of laminar flow from plug flow. It is the dimensionless ratio of $D_{\text {disp }} / V L$ where $L$ is the reactor length. He defines a critical limit of 0.01 for this ratio which assures the deviation to be less than $5 \%$. This means that if a reactor is designed in such a way that the vessel dispersion number is less than 0.01, it can be modeled by plug flow simulation, otherwise the effect of axial and radial diffusion cannot be neglected. 
In order to analyze the effective dispersion coefficient on the fuel pyrolysis in UVa tube reactor, the molecular diffusion coefficients in 1D governing equations were modified to include the Taylor-Aris dispersion (from Eq. 2.12). Figure 2.6 compares the results from the ideal plug flow model with the results from the modified model including effective dispersion. There predictions show no significant difference and can be neglected. Therefore, the effect of this parameter is not studied further in this work.

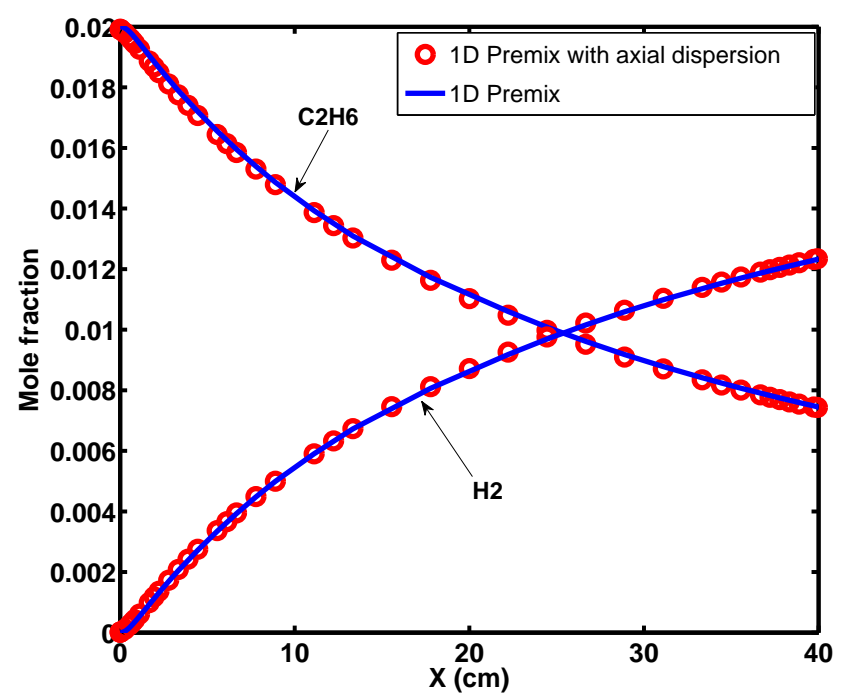

Figure 2.6: Ethane pyrolysis in 1D flow reactor simulation with and without TaylorAris dispersion coefficient.

\subsection{Detailed Chemical Kinetic Models}

Detailed kinetic mechanisms try to include all the relevant elementary reaction pathways usually tailored for a particular fuel. The hydrogen oxidation mechanism is the basis of any hydrocarbon reaction mechanism [27]. This importance of hydrogen reaction to a variety of combustion application, is the main reason why historically there has been a large number of literature, studying and describing hydrogen combustion mechanism [12,28-39]. 
A lot of combustion research groups have been working to extend the detailed mechanisms to hydrocarbon fuel oxidation and pyrolysis regimes. A number of chemical models have been published for different hydrocarbons, such as methane [40-42], methanol [43,44], ethane [45,46], ethylene [47], ethanol [48], propene [49], propane [50], and heavier alkane, alkene, and aromatics containing more than four carbon

atoms $[9,51,52]$. The mechanisms for heavy fuel compounds consist of hundreds of intermediate species and thousands of elementary reactions to describe the reaction pathways and predict the relevant combustion chemistry properties.

These generated mechanisms are validated against a wide range of experimental data for varied operating conditions, including shock-tube ignition delay times [53-57], shock tube speciation profiles [58,59], laminar flame speeds [60-63], laminar flow reactors [64], turbulent plug flow reactors [12,48], perfectly-stirred reactors [65-67], and burner stabilized flames [68].

\subsubsection{Detailed Mechanisms used for Model Prediction}

In the present work, three to four detailed mechanisms were selected for the prediction of each fuel pyrolysis in the UVa tube reactor experiments.

\section{Ethane}

For ethane pyrolysis they are: 1) Wang et al. (JetSurF 2.0) model [69]. This model consists of 348 species and 2163 reactions. It includes the high-temperature chemistry of all n-alkanes up to n-dodecane and related cycloalkanes and aromatics. This model is an extension to the base model USC-Mech II [70], which has 111 species and 784 reactions, that describes the oxidation of $\mathrm{H} 2$ and $\mathrm{CO}$ and the high-temperature chemistry of C1-C4 hydrocarbons. 2) Naik and Dean model [46] which consists of 181 species and 2066 reactions. This model is developed specifically for ethane pyrolysis and oxidation and is validated against shock tube ethane pyrolysis and oxidation ex- 
periments by Tranter et al. [71,72]. 3) AramcoMech2.0 model [73] which consists of 493 species and 2716 reactions. This model is developed by the Combustion Chemistry Centre at NUI Galway. The model is developed in a hierarchical way to describe the kinetics and thermochemical properties of hydrogen oxidation and C1-C4 hydrocarbons. It is developed and validated based on wide range of experiments including recent studies by Burke et al. [57,67].

\section{n-Butane}

For n-buane pyrolysis the three models selected are: 1) Wang et al. model [69]. 2) AramcoMech2.0 model [73]. 3) Pyun et al. model [58] which is a modified version of JetSurF 2.0 model tuned for n-butane pyrolysis. In their study, methane and ethylene concentration were measurements for n-butane pyrolysis behind reflected shock waves at temperatures from 1254 to $1565 \mathrm{~K}$. By comparing their measured data with predictions from mechanism by Wang et al. [69], they suggested a few modifications to this mechanism to improve agreement between the model predictions and measured data for $\mathrm{CH}_{4}$ and $\mathrm{C}_{2} \mathrm{H}_{4}$ concentrations. Specifically, the two important initiation reactions in n-butane decomposition that were determined by the reaction pathway analysis at initial time steps, i.e. $\mathrm{C}_{4} \mathrm{H}_{10}(+\mathrm{M}) \rightarrow 2 \mathrm{C}_{2} \mathrm{H}_{5}(+\mathrm{M})$ and $\mathrm{C}_{4} \mathrm{H}_{10}$ $(+\mathrm{M}) \rightarrow \mathrm{n}-\mathrm{C}_{3} \mathrm{H}_{7}+\mathrm{CH}_{3}(+\mathrm{M})$, were modified to the reaction rate values measured by Oehlschlaeger et al. [74]. Pyun et al. [58] also suggested to increase the reaction rate of $\mathrm{C}_{4} \mathrm{H}_{10}+\mathrm{CH}_{3} \rightarrow \mathrm{sC}_{4} \mathrm{H}_{9}+\mathrm{CH}_{4}$ by factor of 2 and decrease the reaction rate of $\mathrm{C}_{4} \mathrm{H}_{10}+\mathrm{H} \rightarrow \mathrm{sC}_{4} \mathrm{H}_{9}+\mathrm{H}_{2}$ by a factor of 2 to improve the prediction of $\mathrm{CH}_{4}$ and $\mathrm{C}_{2} \mathrm{H}_{4}$ concentrations.

\section{n-Dodecane}

For n-dodecane pyrolysis four models are selected, which are: 1) Wang et al. model [69]. 2) Banerjee et al. model [19] which consist of 196 species and 1478 reac- 
tions. This is an optimized model based on Wang et al. model. The flow reactor data for n-dodecane pyrolysis and oxidation (reported in [19]) were used to perform uncertainty minimization and improve model predictions. The result is shown there that the predictions of optimized model are improved significantly for n-dodecane combustion data. 3) Ranzi et al. model [75] of the CRECK group at Milano, Italy. This model consists of 451 species and 17848 reactions. This detailed model is developed using a lumping procedure to reduce the number of species and reactions present in the automatically generated mechanism while keeping the accuracy of the prediction properties. For example, global reaction NC12H26 $\rightarrow$ $0.5 \mathrm{C} 2 \mathrm{H} 5+0.5 \mathrm{NC} 3 \mathrm{H} 7+0.5 \mathrm{NC} 7 \mathrm{H} 15+0.5 \mathrm{NC} 12 \mathrm{H} 25$, is one of the n-dodecane pyrolysis reactions used in this model with non-integer stoichiometric coefficients. In the kinetic mechanisms with elementary reactions, fraction of molecules cannot participate as a reactant or product, so they can only take integer values for stoichiometric coefficients. Buy employing the lumping procedure with global reactions, the model is reduced without sacrificing the predictive capabilities. This mechanism can be downloaded from CRECK website at http://creckmodeling.chem.polimi.it. 4) Mze-Ahmed et al. model [76] developed by CNRS group led by Philippe Dagaut at Orleans, France. It consists of 1377 species and 6014 reactions (5864 reversible reactions). This detailed chemical kinetic model is developed based on a recent ndodecane and n-undecane oxidation kinetics studies in CNRS jet-stirred reactor. The JSR experiments were at high pressure $(\mathrm{P}=10 \mathrm{bar})$, temperatures ranging from 550 to $1150 \mathrm{~K}$, constant residence time of $1 \mathrm{~s}$, and for three equivalence ratios $(0.5,1.0$, and 2.0) [76]. 


\subsection{Model Results Comparison with Experiment}

\subsubsection{Ethane}

Figures 2.7-2.14 show the comparison of three chemical kinetic models with the experimental data from the UVa tube reactor for ethane pyrolysis. These figures show the major species mole fraction profiles as a function of reactor temperature for residence times from $10 \mathrm{~ms}$ to $90 \mathrm{~ms}$. There are two data points for the experimental result, $1050 \mathrm{~K}$ and $1100 \mathrm{~K}$. The error bar indicates the uncertainty associated with the data. The main sources of experimental measurements uncertainty are data repeatability, flow controllers, and GC calibration uncertainties. The model predictions are based on the 0D constant temperature calculation. The predictions from AramcoMech2.0 model are in better agreement with the experimental data for most of the major species compared to the Wang et al. model and Naik and Dean Model. The details of the results are recently submitted for publication in [20]. 

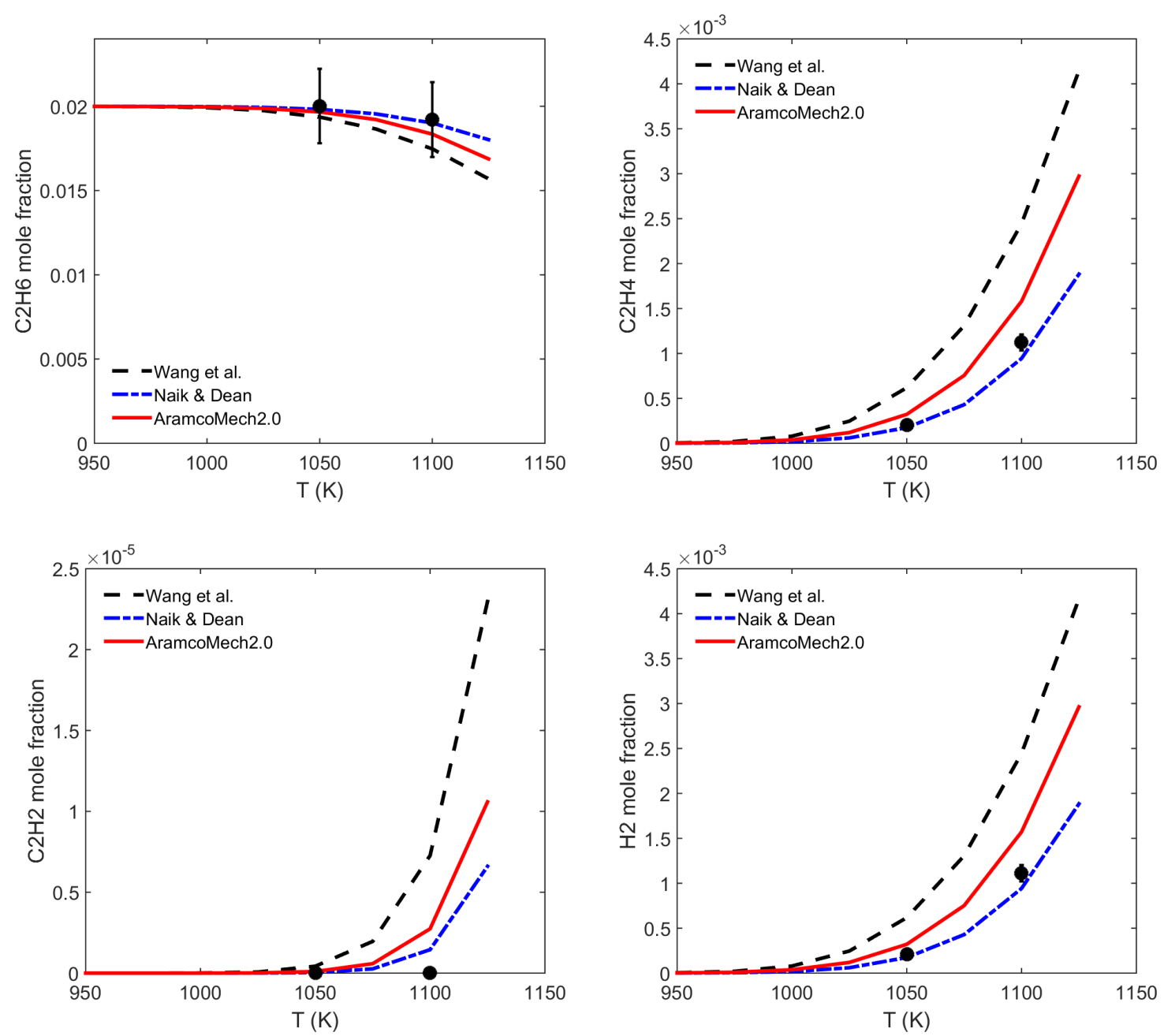

Figure 2.7: Species mole fraction as a function of temperature for $2 \%$ ethane pyrolysis in the atmospheric pressure tube reactor at $10 \mathrm{~ms}$ residence time. Symbols are the experimental data with error bar indicating the uncertainty of the data measurements. Lines are the model prediction using the three different chemistry mechanisms. 

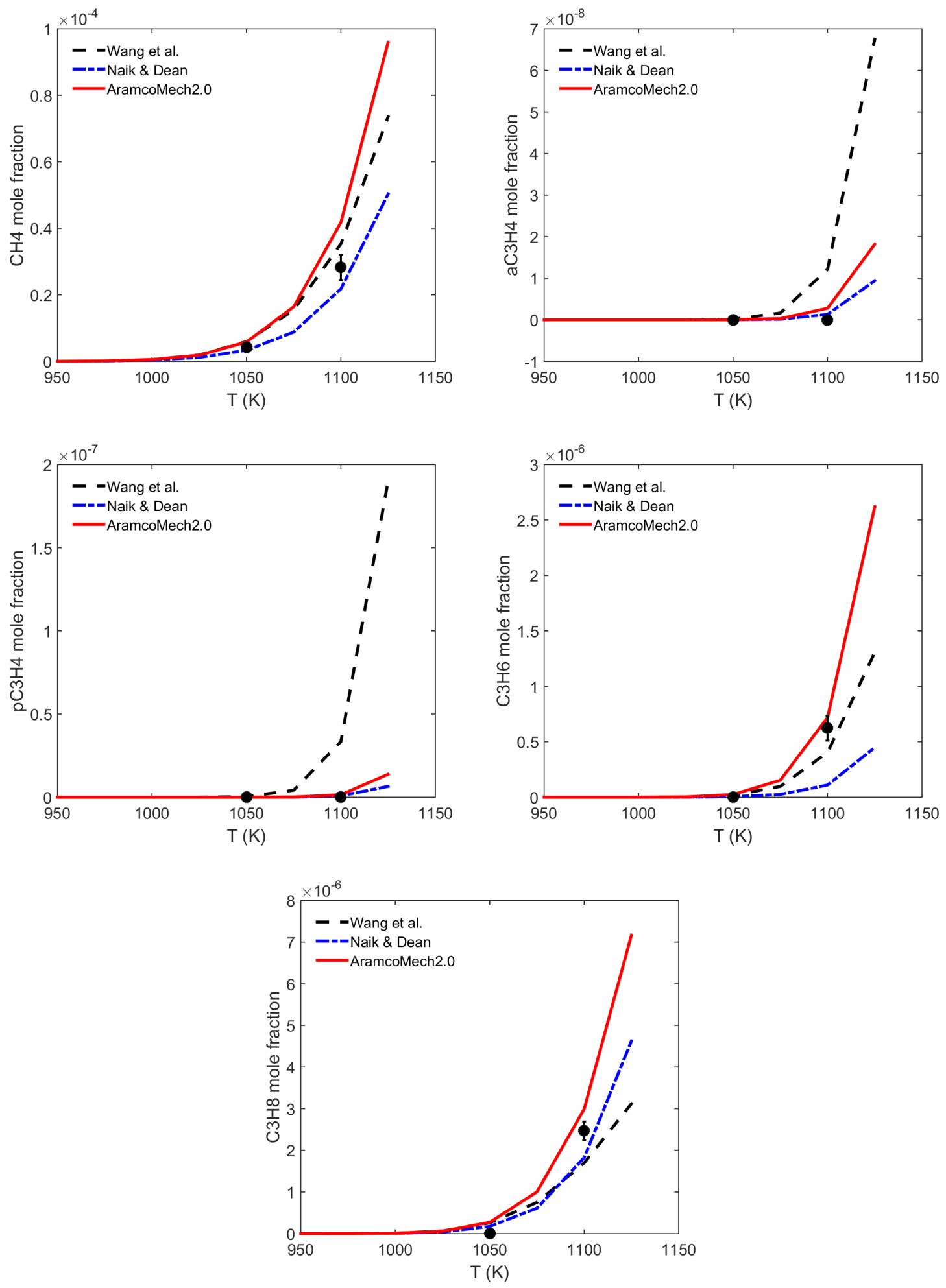

Figure 2.8: Species mole fraction as a function of temperature for $2 \%$ ethane pyrolysis in the atmospheric pressure tube reactor at $10 \mathrm{~ms}$ residence time. Symbols are the experimental data with error bar indicating the uncertainty of the data measurements. Lines are the model prediction using the three different chemistry mechanisms. 

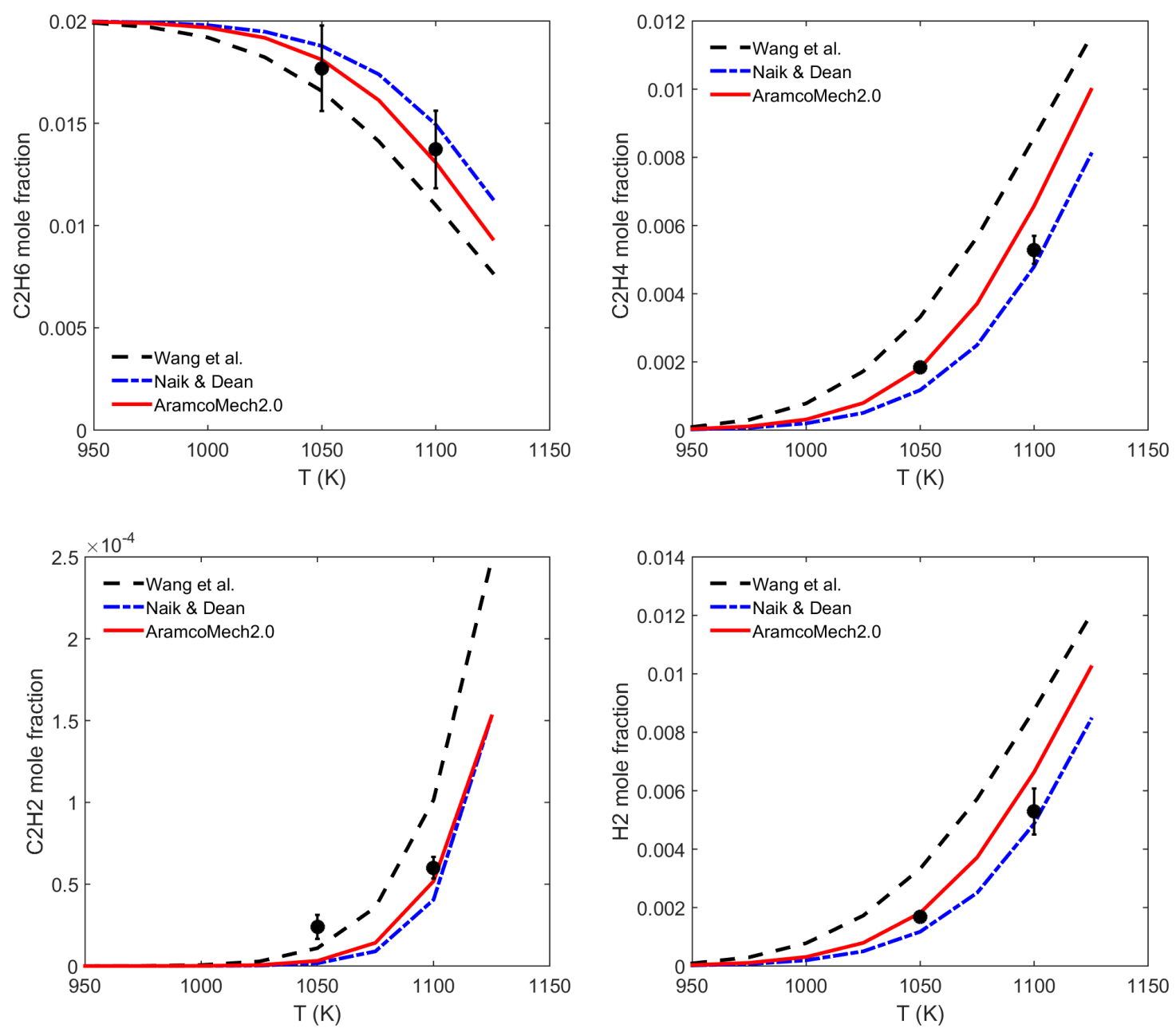

Figure 2.9: Species mole fraction as a function of temperature for $2 \%$ ethane pyrolysis in the atmospheric pressure tube reactor at $50 \mathrm{~ms}$ residence time. Symbols are the experimental data with error bar indicating the uncertainty of the data measurements. Lines are the model prediction using the three different chemistry mechanisms. 

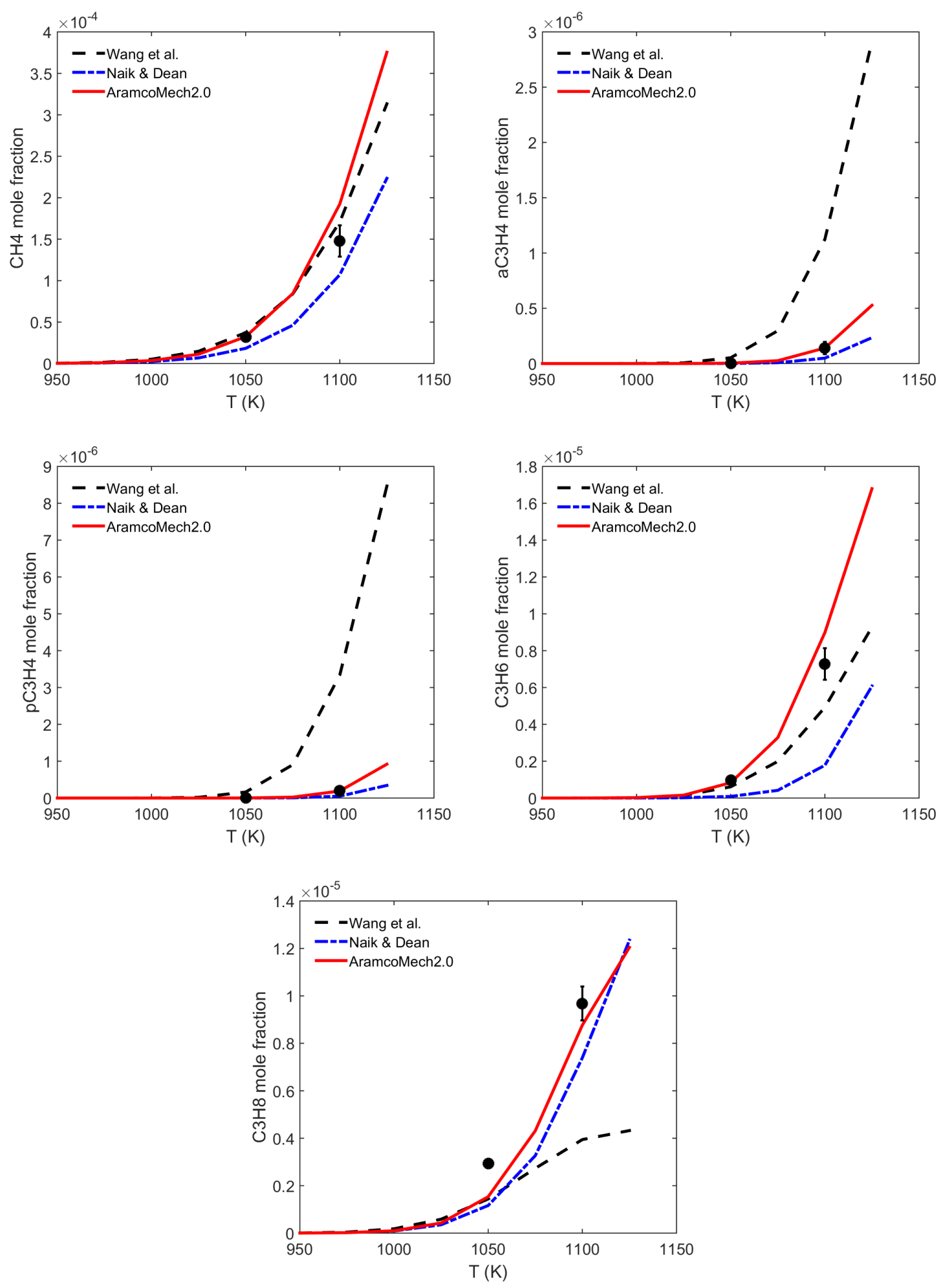

Figure 2.10: Species mole fraction as a function of temperature for $2 \%$ ethane pyrolysis in the atmospheric pressure tube reactor at $50 \mathrm{~ms}$ residence time. Symbols are the experimental data with error bar indicating the uncertainty of the data measurements. Lines are the model prediction using the three different chemistry mechanisms. 

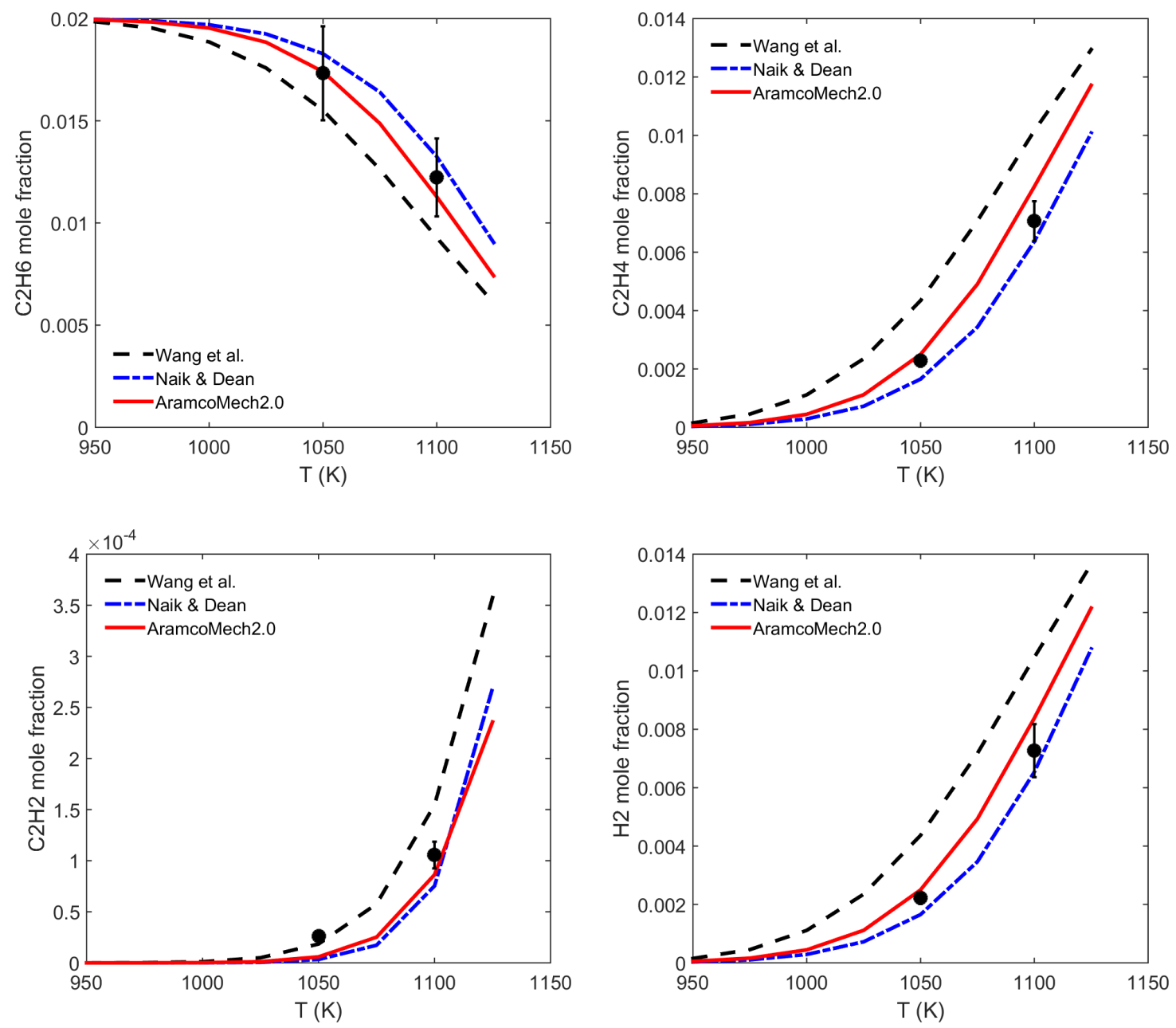

Figure 2.11: Species mole fraction as a function of temperature for $2 \%$ ethane pyrolysis in the atmospheric pressure tube reactor at $70 \mathrm{~ms}$ residence time. Symbols are the experimental data with error bar indicating the uncertainty of the data measurements. Lines are the model prediction using the three different chemistry mechanisms. 

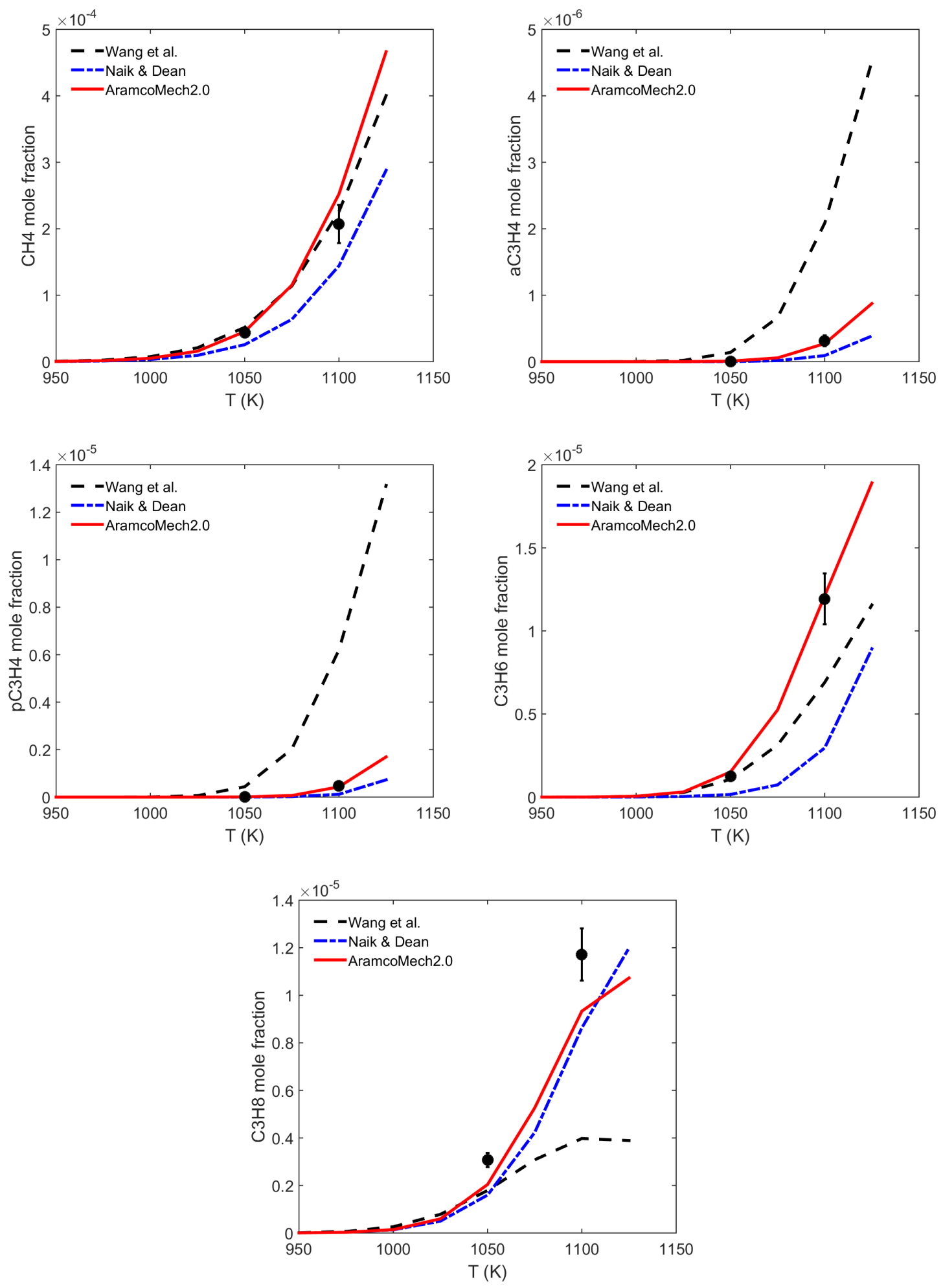

Figure 2.12: Species mole fraction as a function of temperature for $2 \%$ ethane pyrolysis in the atmospheric pressure tube reactor at $70 \mathrm{~ms}$ residence time. Symbols are the experimental data with error bar indicating the uncertainty of the data measurements. Lines are the model prediction using the three different chemistry mechanisms. 

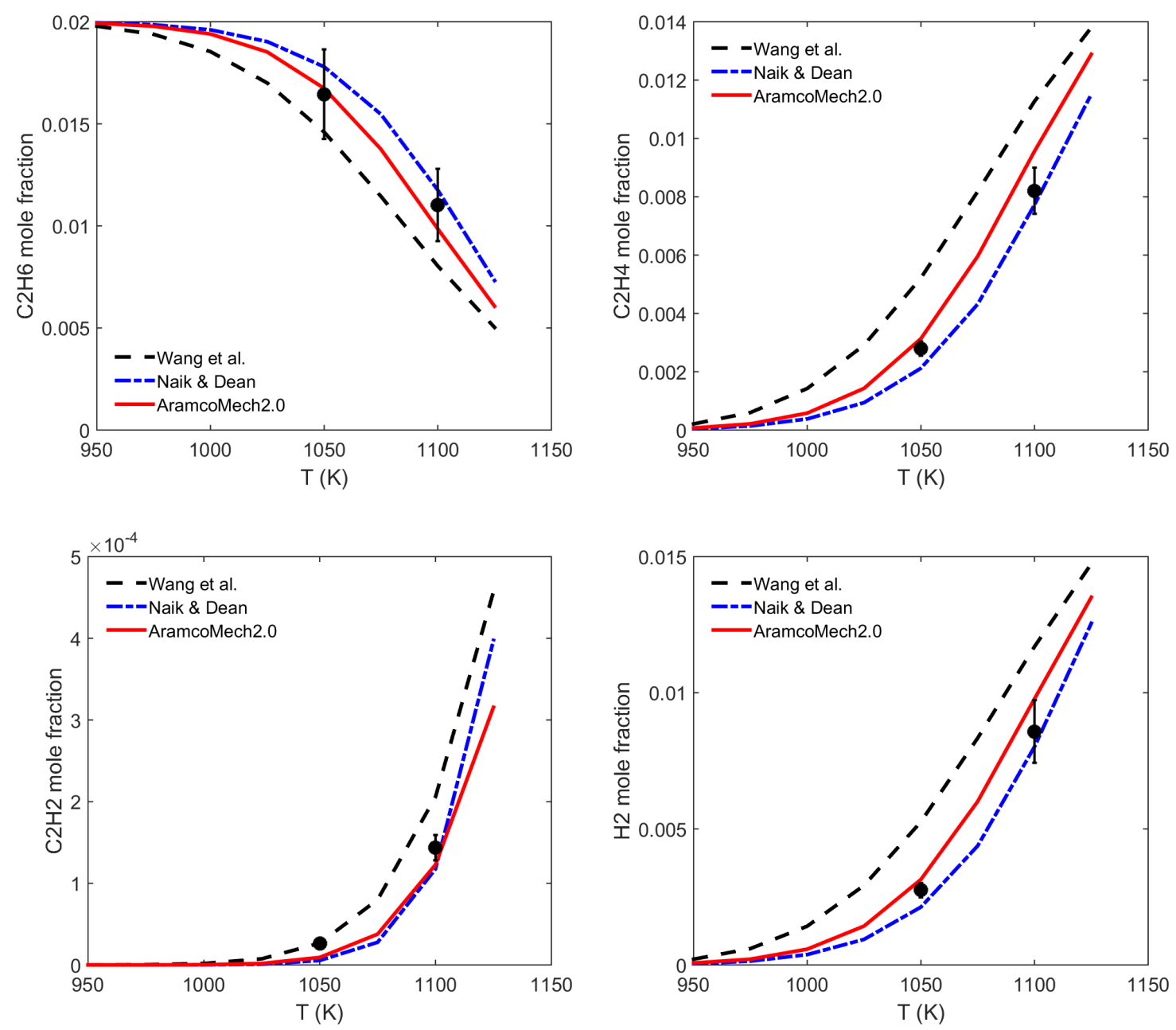

Figure 2.13: Species mole fraction as a function of temperature for $2 \%$ ethane pyrolysis in the atmospheric pressure tube reactor at $90 \mathrm{~ms}$ residence time. Symbols are the experimental data with error bar indicating the uncertainty of the data measurements. Lines are the model prediction using the three different chemistry mechanisms. 

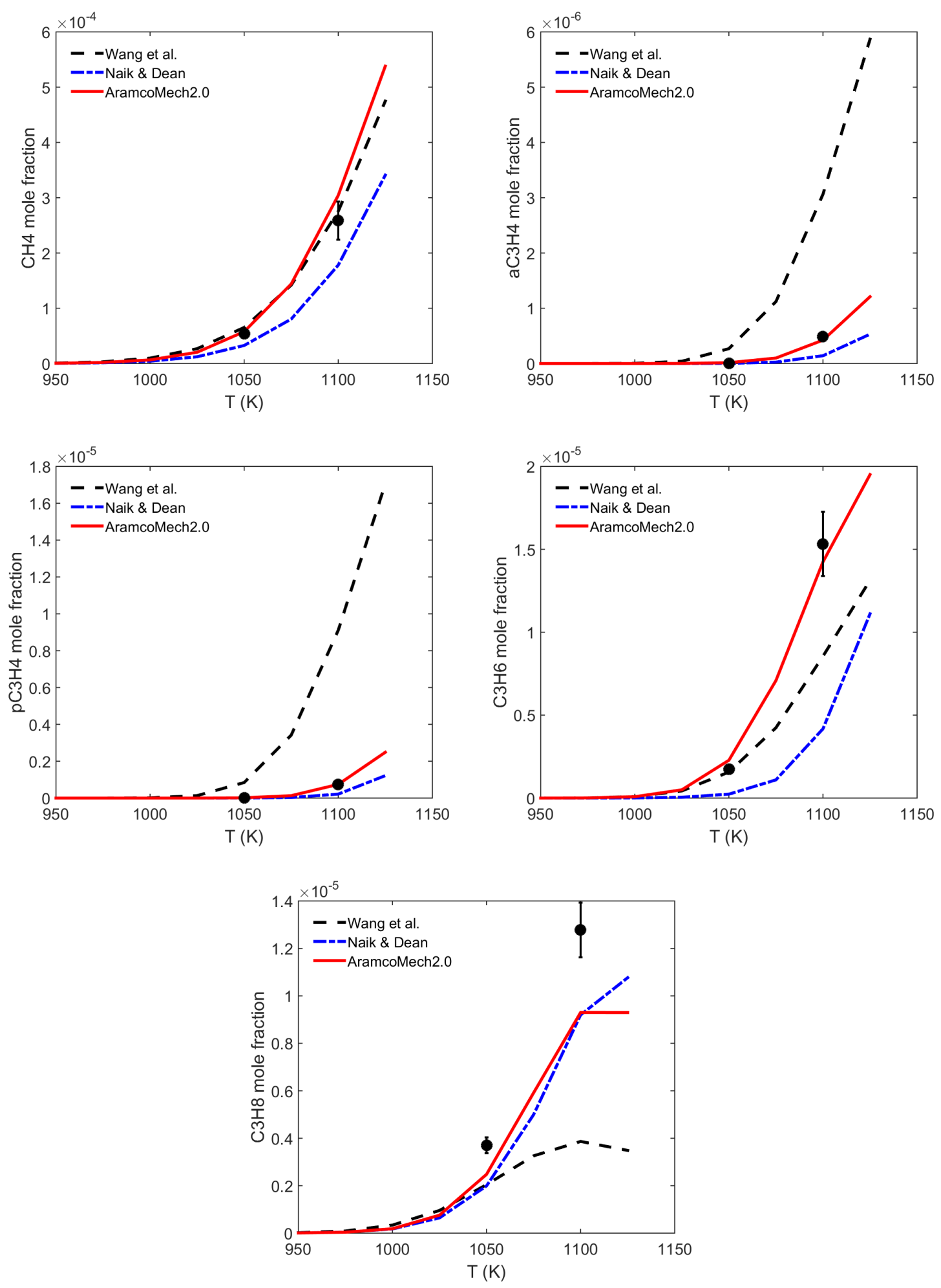

Figure 2.14: Species mole fraction as a function of temperature for $2 \%$ ethane pyrolysis in the atmospheric pressure tube reactor at $90 \mathrm{~ms}$ residence time. Symbols are the experimental data with error bar indicating the uncertainty of the data measurements. Lines are the model prediction using the three different chemistry mechanisms. 


\subsection{2 n-Butane}

Figures 2.15-2.22 show the comparison of three chemical kinetic models with the experimental data from the UVa tube reactor for n-butane pyrolysis. These figures show the major species mole fraction profiles as a function of reactor temperature for residence times from $30 \mathrm{~ms}$ to $90 \mathrm{~ms}$. There are two data points for the experimental result, $1050 \mathrm{~K}$ and $1100 \mathrm{~K}$. The error bar indicates the uncertainty associated with the data. The model predictions are based on the 0D constant temperature calculation. Although the Pyun et al. model has optimized Wang et al. model for n-butane chemistry by modifying only 4 reactions, these figures show a large difference between the prediction result from Pyun and Wang model. It can be noticed that the results from all the models vary on how they predict the experimental data. AramcoMech2.0 model predicts the ethylene and hydrogen and n-butane mole fraction better than the other two models. Wang et al. model predicts the acetylene $(\mathrm{C} 2 \mathrm{H} 2)$, allene/propadiene (aC3H4), and propyne ( $\mathrm{pC} 3 \mathrm{H} 4)$ mole fraction better compared to the other models. Pyun et al. model predicts methane (CH4) mole fraction, and at lower temperature propene $(\mathrm{C} 3 \mathrm{H} 6)$ mole fraction better than the other two models. These results and the detail discussions are recently submitted for publication in [20]. 

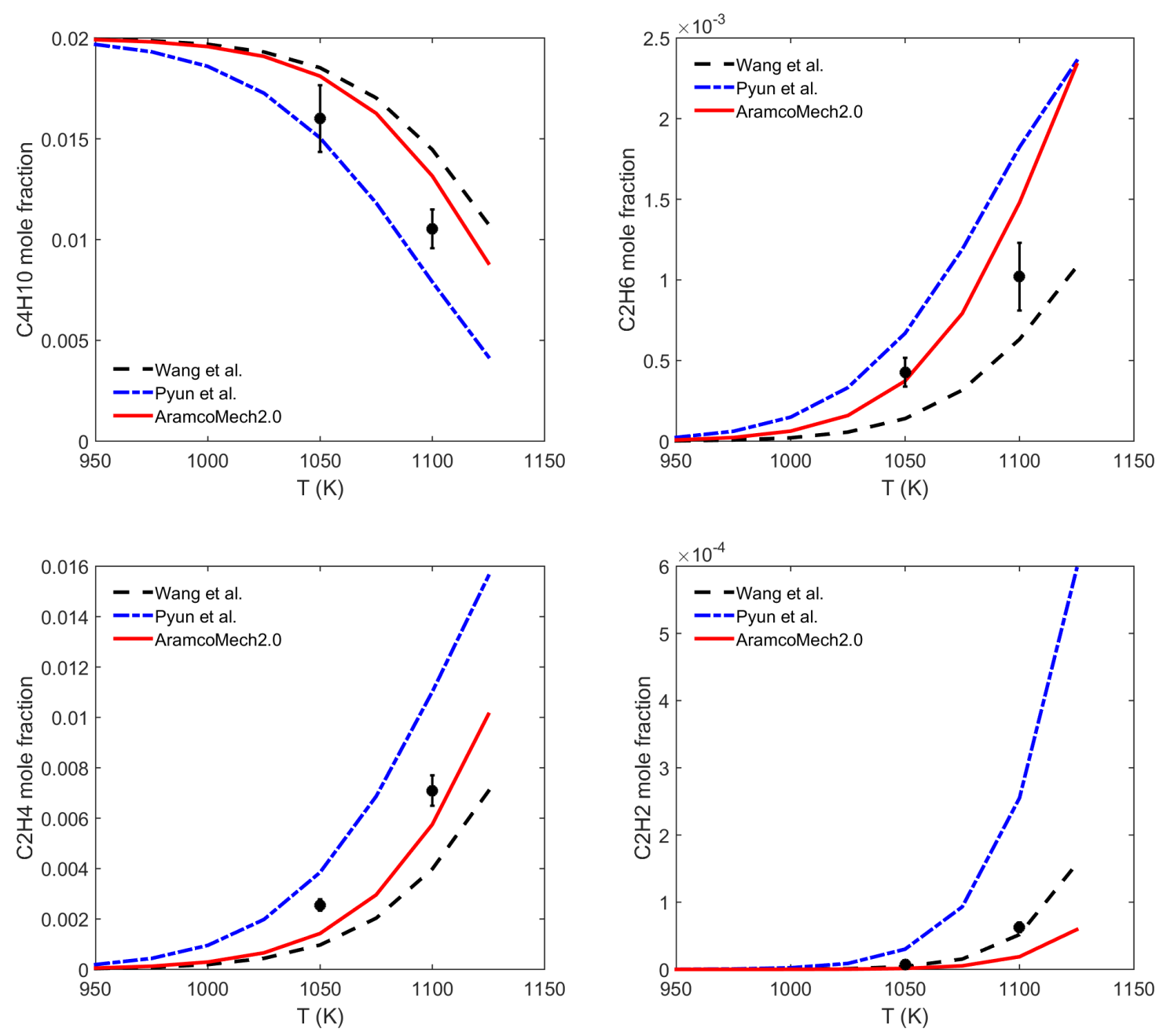

Figure 2.15: Species mole fraction as a function of temperature for $2 \%$ n-butane pyrolysis in the atmospheric pressure tube reactor at $30 \mathrm{~ms}$ residence time. Symbols are the experimental data with error bar indicating the uncertainty of the data measurements. Lines are the model prediction using the three different chemistry mechanisms. 

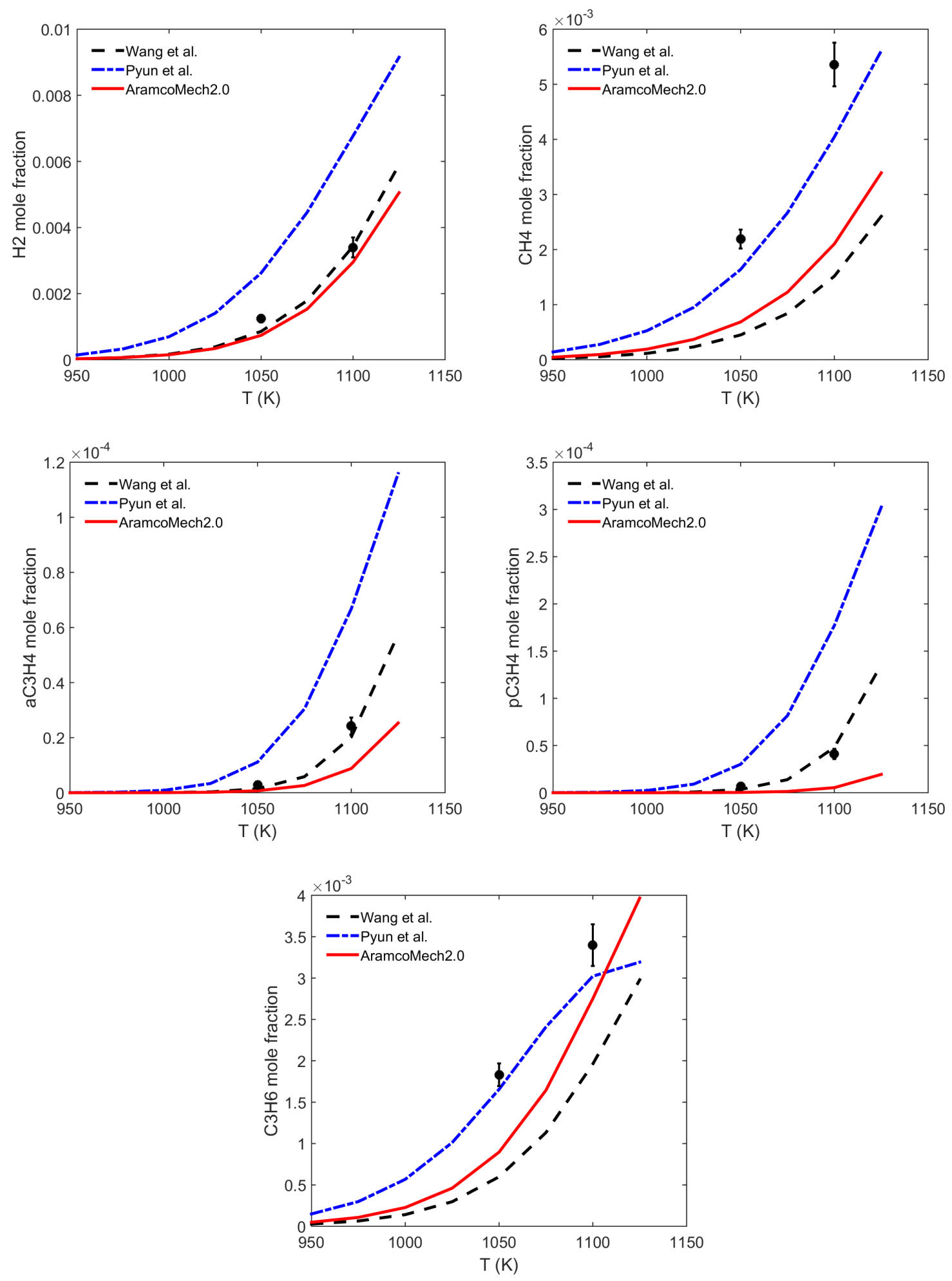

Figure 2.16: Species mole fraction as a function of temperature for $2 \%$ n-butane pyrolysis in the atmospheric pressure tube reactor at $30 \mathrm{~ms}$ residence time. Symbols are the experimental data with error bar indicating the uncertainty of the data measurements. Lines are the model prediction using the three different chemistry mechanisms. 

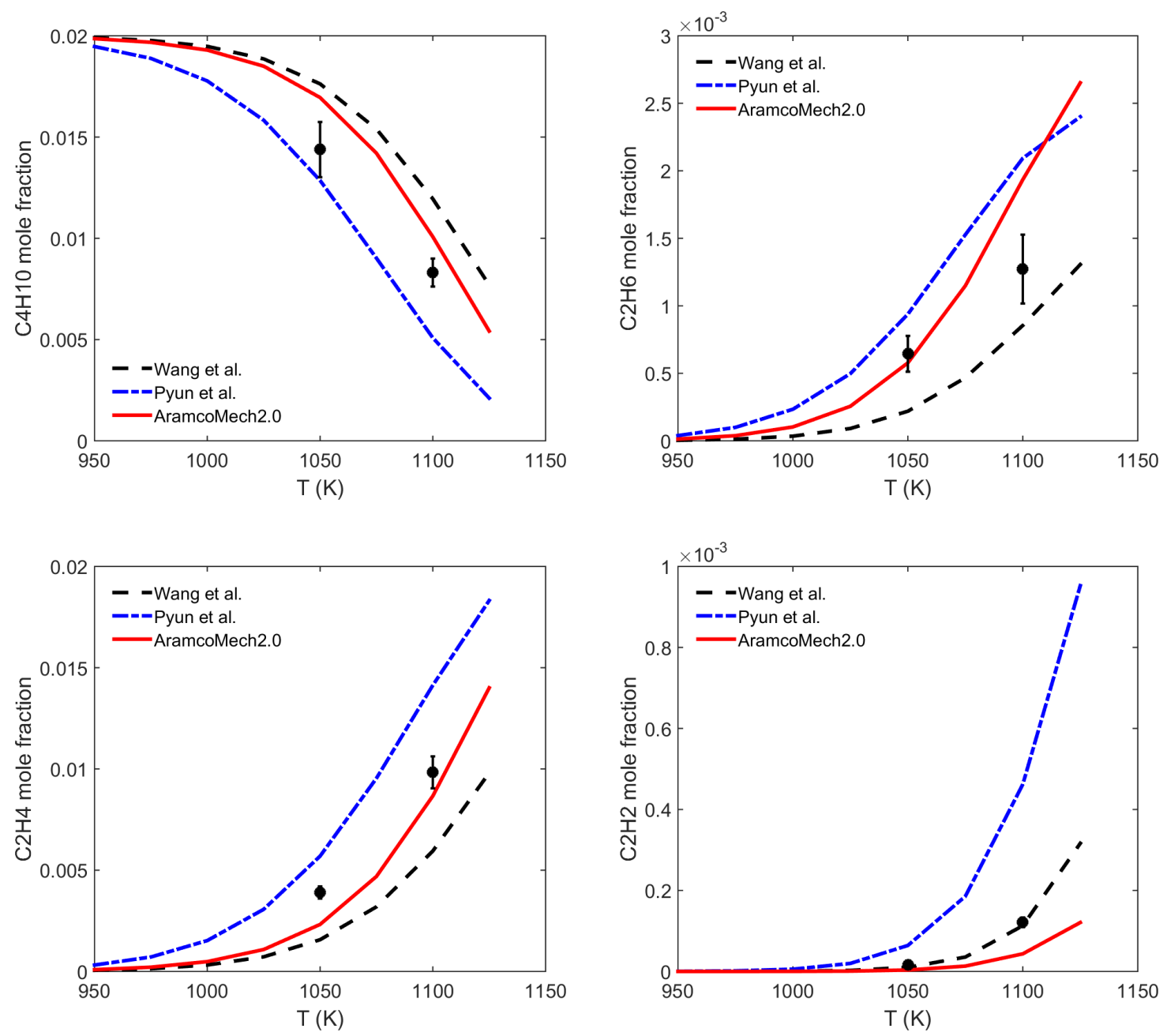

Figure 2.17: Species mole fraction as a function of temperature for $2 \%$ n-butane pyrolysis in the atmospheric pressure tube reactor at $50 \mathrm{~ms}$ residence time. Symbols are the experimental data with error bar indicating the uncertainty of the data measurements. Lines are the model prediction using the three different chemistry mechanisms. 

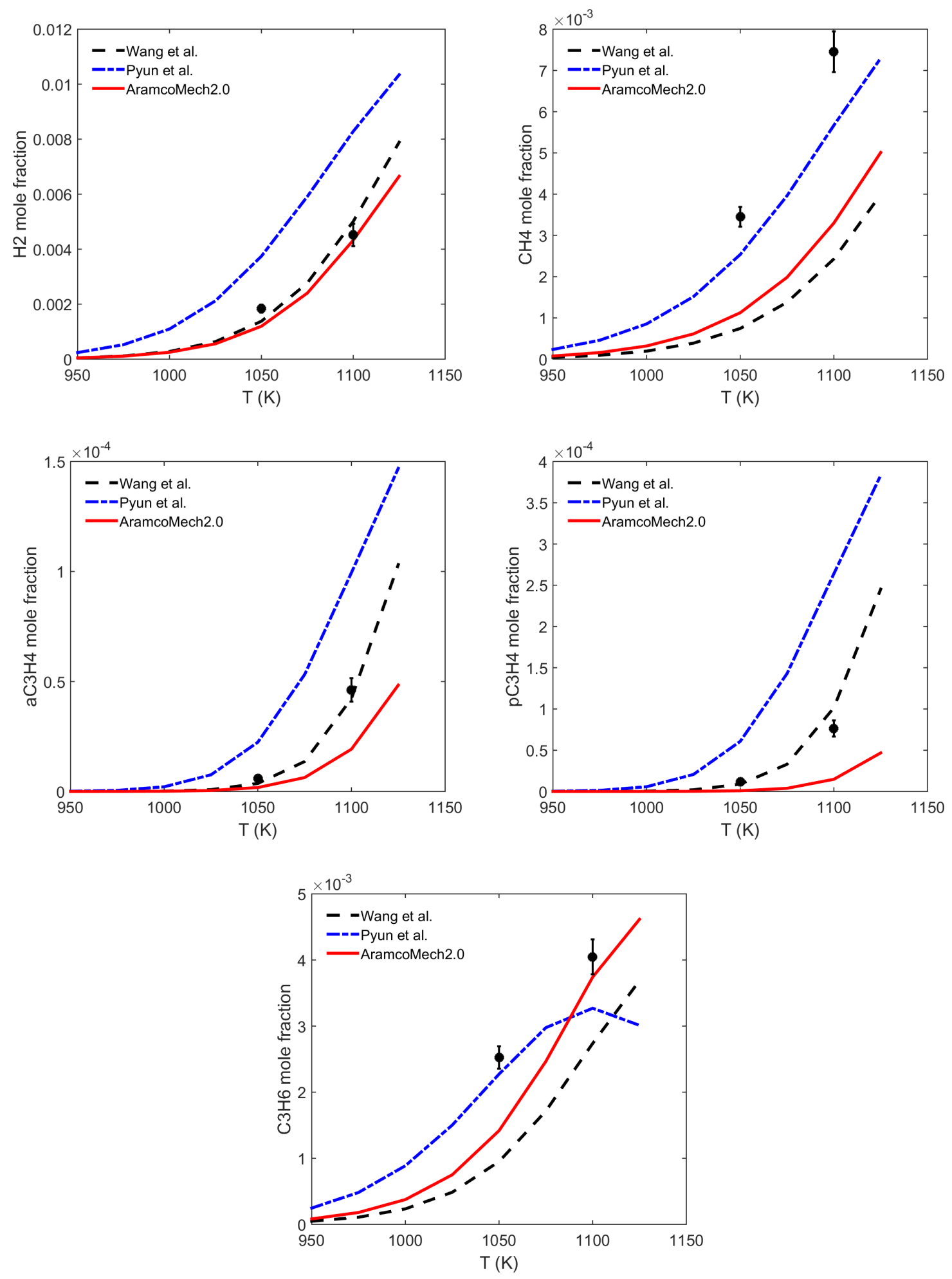

Figure 2.18: Species mole fraction as a function of temperature for $2 \%$ n-butane pyrolysis in the atmospheric pressure tube reactor at $50 \mathrm{~ms}$ residence time. Symbols are the experimental data with error bar indicating the uncertainty of the data measurements. Lines are the model prediction using the three different chemistry mechanisms. 

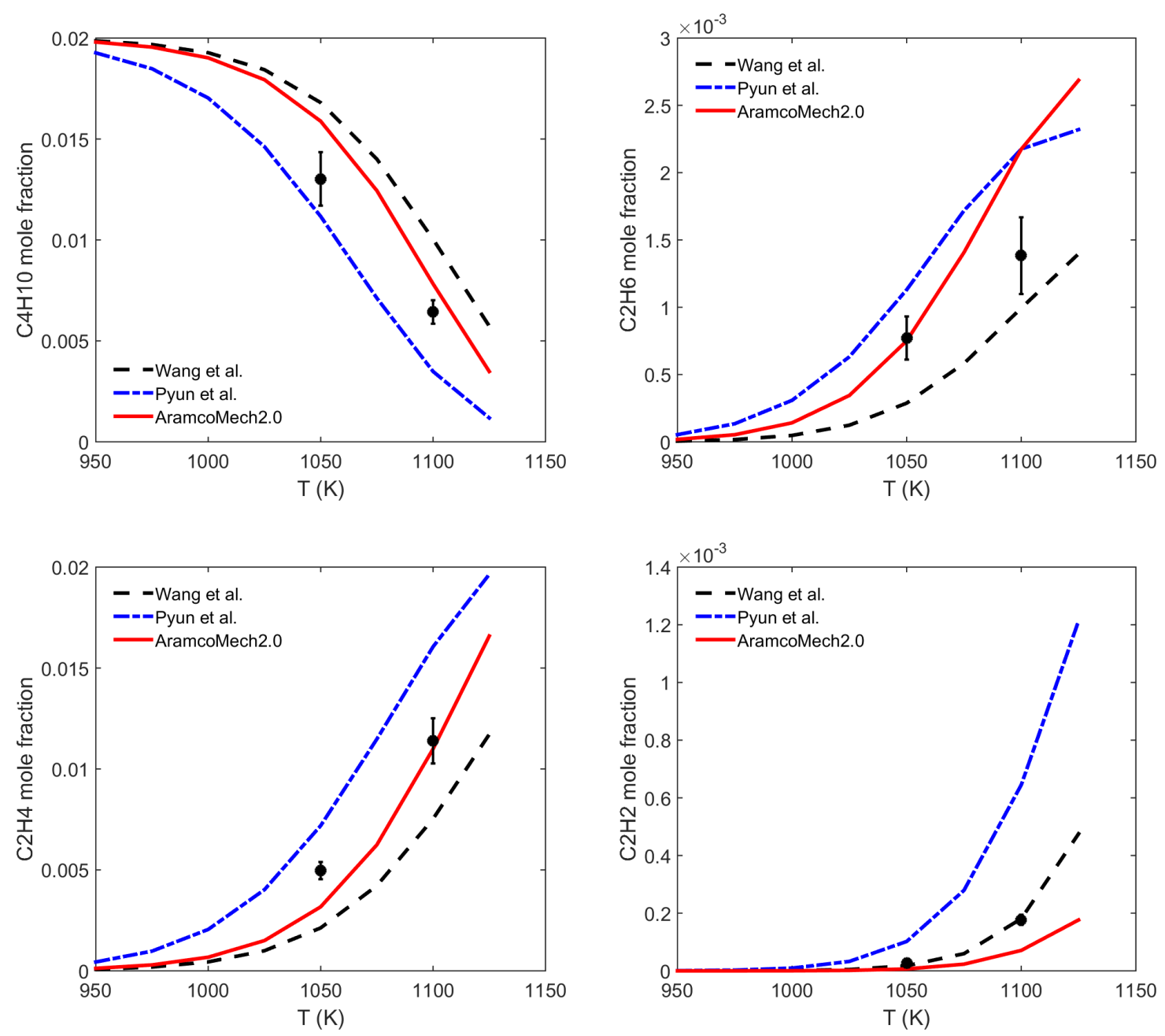

Figure 2.19: Species mole fraction as a function of temperature for $2 \%$ n-butane pyrolysis in the atmospheric pressure tube reactor at $70 \mathrm{~ms}$ residence time. Symbols are the experimental data with error bar indicating the uncertainty of the data measurements. Lines are the model prediction using the three different chemistry mechanisms. 

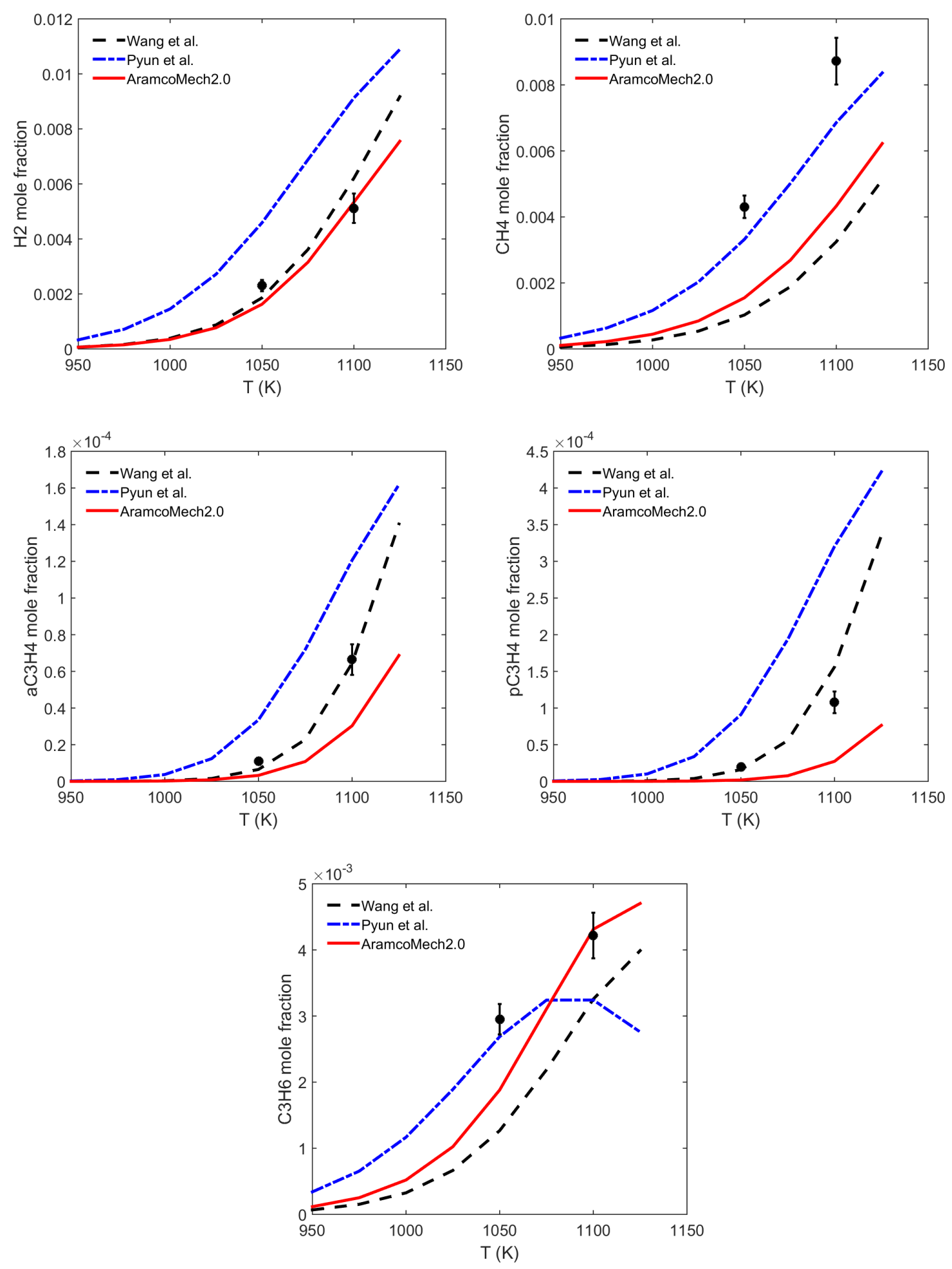

Figure 2.20: Species mole fraction as a function of temperature for $2 \%$ n-butane pyrolysis in the atmospheric pressure tube reactor at $70 \mathrm{~ms}$ residence time. Symbols are the experimental data with error bar indicating the uncertainty of the data measurements. Lines are the model prediction using the three different chemistry mechanisms. 

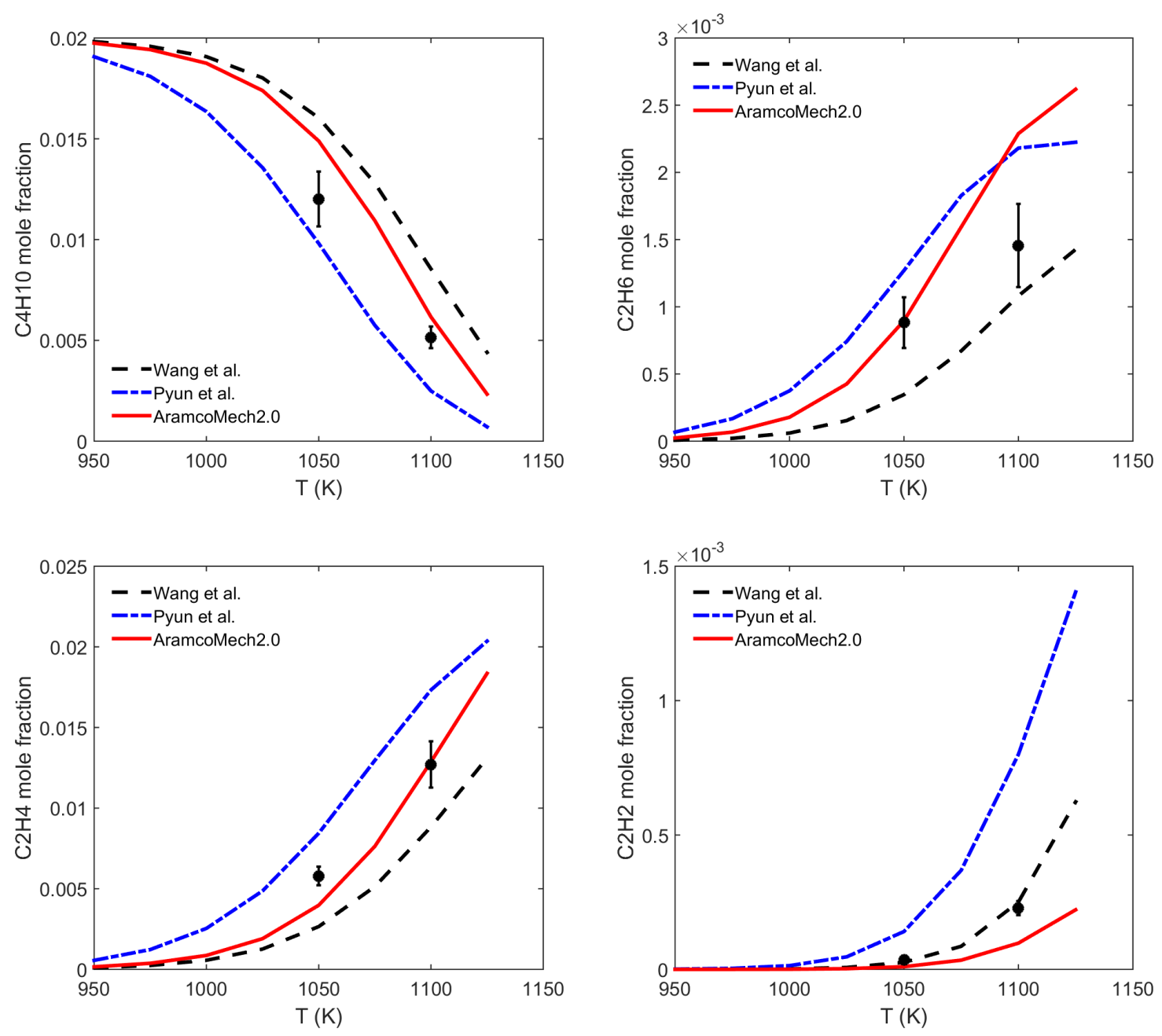

Figure 2.21: Species mole fraction as a function of temperature for $2 \%$ n-butane pyrolysis in the atmospheric pressure tube reactor at $90 \mathrm{~ms}$ residence time. Symbols are the experimental data with error bar indicating the uncertainty of the data measurements. Lines are the model prediction using the three different chemistry mechanisms. 

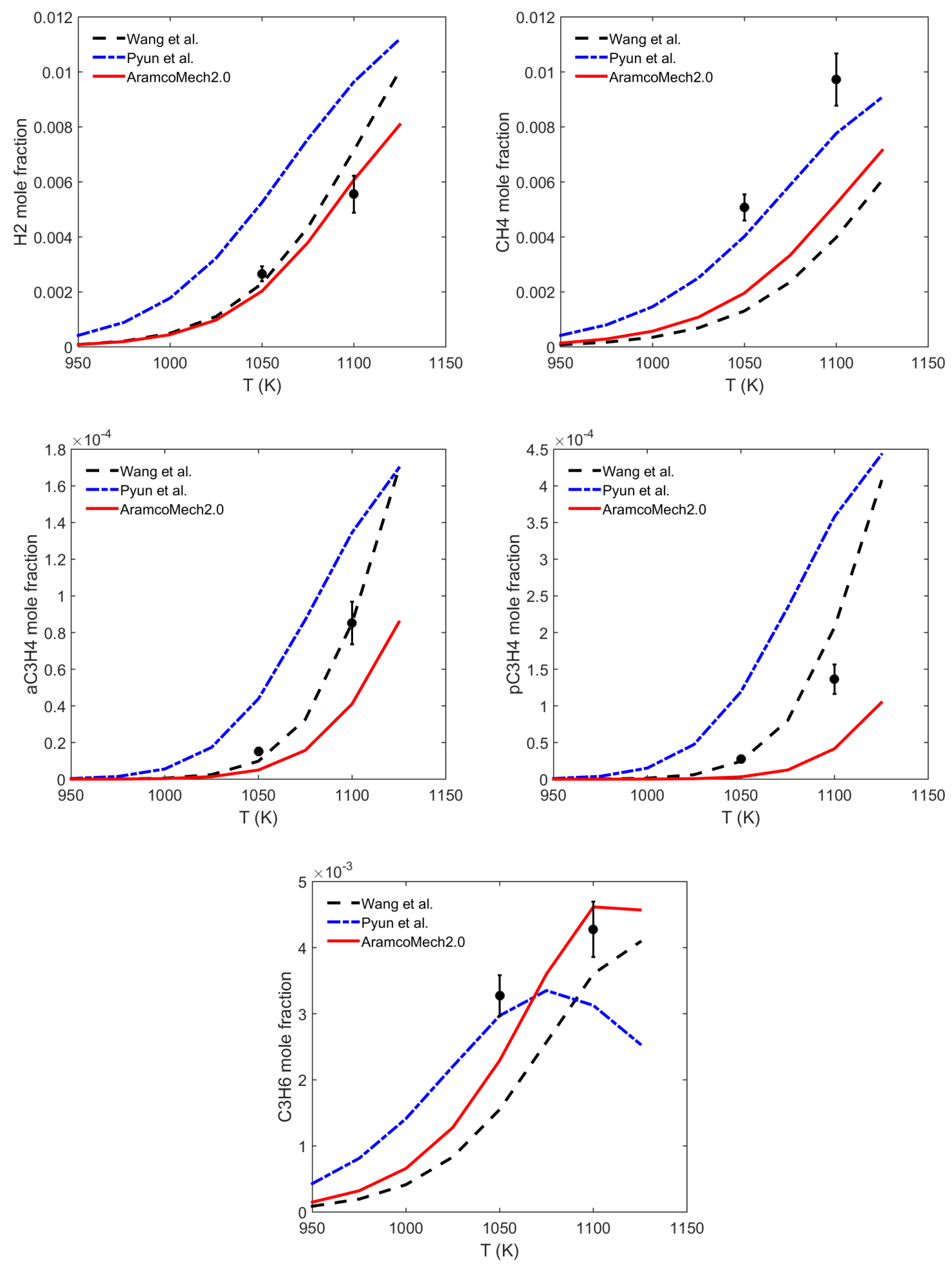

Figure 2.22: Species mole fraction as a function of temperature for $2 \%$ n-butane pyrolysis in the atmospheric pressure tube reactor at $90 \mathrm{~ms}$ residence time. Symbols are the experimental data with error bar indicating the uncertainty of the data measurements. Lines are the model prediction using the three different chemistry mechanisms. 


\subsection{3 n-Dodecane}

For n-dodecane pyrolysis, the product species from the reactor are not just small hydrocarbons anymore. There are larger hydrocarbons with C5 and higher, produced in the fuel cracking process. These larger species are not measured in the GC/GCMS system used for our experiments. Our measurement devices can only measure species smaller than n-butane (H2, O2, CO, CO2, and C1-C4 hydrocarbons). There is definitely an uncertainty associated with the data measurements when the larger species (C5-C12) available in the sampled mixture are not measured in the GC system.

To quantify the effect of this uncertainty and find the correct mole fraction of the measured species, the mole fraction for the unmeasured larger species are calculated and taken into account to find the correct mole fraction for $\mathrm{C} 1-\mathrm{C} 4$ species. The calculations shows that the change due to the error of not measuring large hydrocarbon concentrations is found to be less than $0.15 \%$ for short residence time $(10 \mathrm{~ms})$ and $0.02 \%$ for long residence time $(60 \mathrm{~ms})$. This calculation proved that the reported mole fraction from the experiments are accurate and the carbon balance issue does not effect the result for the current operating conditions. The main reason is that the mixture is highly diluted (99.75\% volume nitrogen). Even high conversions of ndodecane will not change the mole fraction of nitrogen more than $2 \%$. As a result, the mole fraction of the measured species are not effected by the small amount (volume fraction) of unmeasured species.

Figures 2.23-2.34 show the comparison of four chemical kinetic models with the experimental data from the UVa tube reactor for n-dodecane pyrolysis. These figures show the major species mole fraction profiles as a function of reactor temperature for residence times from $10 \mathrm{~ms}$ to $60 \mathrm{~ms}$. The $10 \mathrm{~ms}$ case has data for three temperatures, $1000 \mathrm{~K}, 1050 \mathrm{~K}$, and $1100 \mathrm{~K}$. The other cases with longer residence times have data points for two temperatures, $1000 \mathrm{~K}$ and $1100 \mathrm{~K}$. The error bar indicates the uncertainty associated with the data. The model predictions are based on the 0D 
constant temperature calculation.

All the four model are predicting the ethylene data with good agreement. However, Mze-Ahmed et al. model is showing the closest match with the data for most of the major species, such as $\mathrm{C} 2 \mathrm{H} 2, \mathrm{H} 2, \mathrm{CH} 4, \mathrm{aC} 3 \mathrm{H} 4$, pC3H4, C4H6 compared to the other three models. Ranzi et al. model performs better in predicting ethane mole fraction. These results with more details are recently submitted for publication in [20].
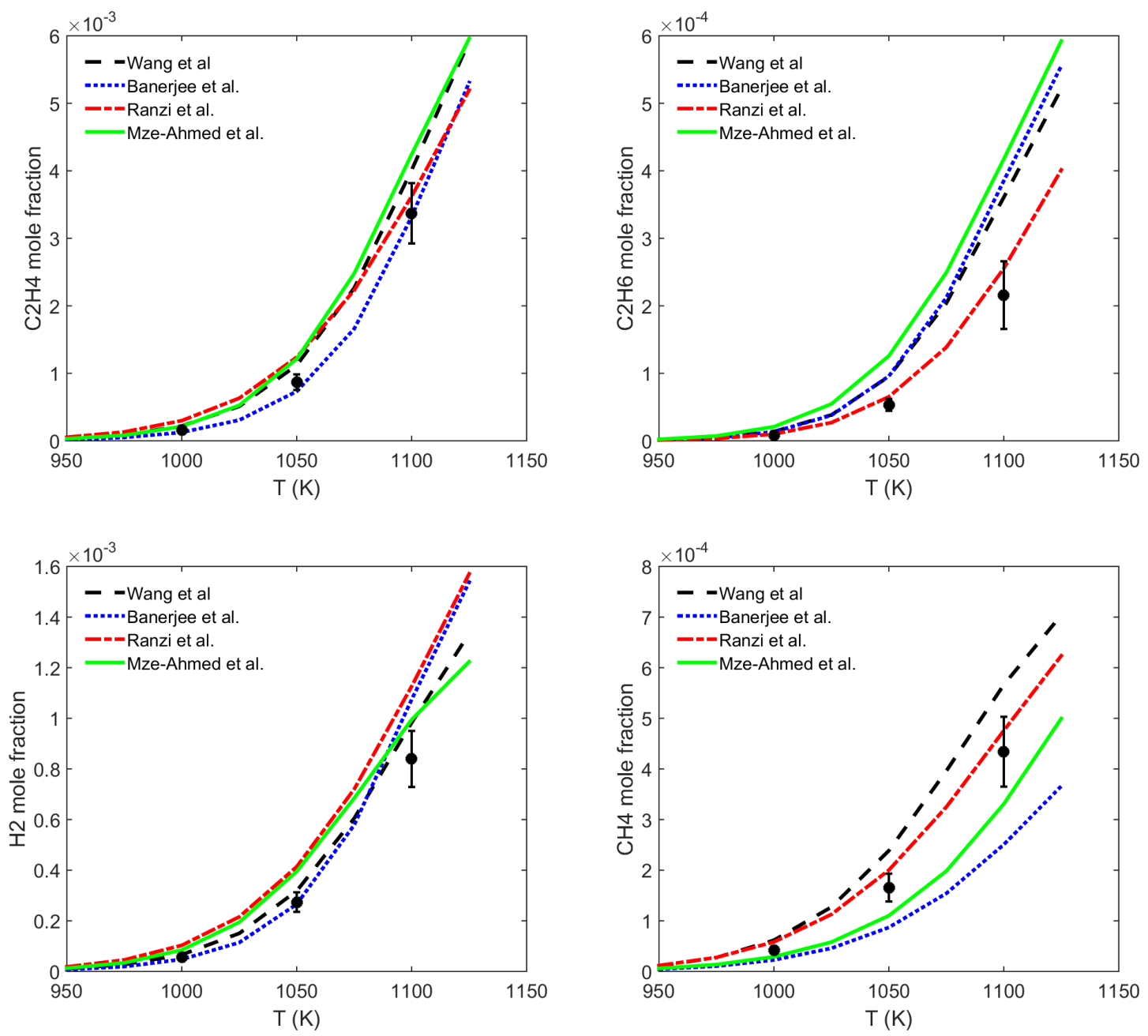

Figure 2.23: Species mole fraction as a function of temperature for $0.25 \%$ n-Dodecane pyrolysis in the atmospheric pressure tube reactor at $10 \mathrm{~ms}$ residence time. Symbols are the experimental data with error bar indicating the uncertainty of the data. Lines are the model prediction using four different chemistry mechanisms. 

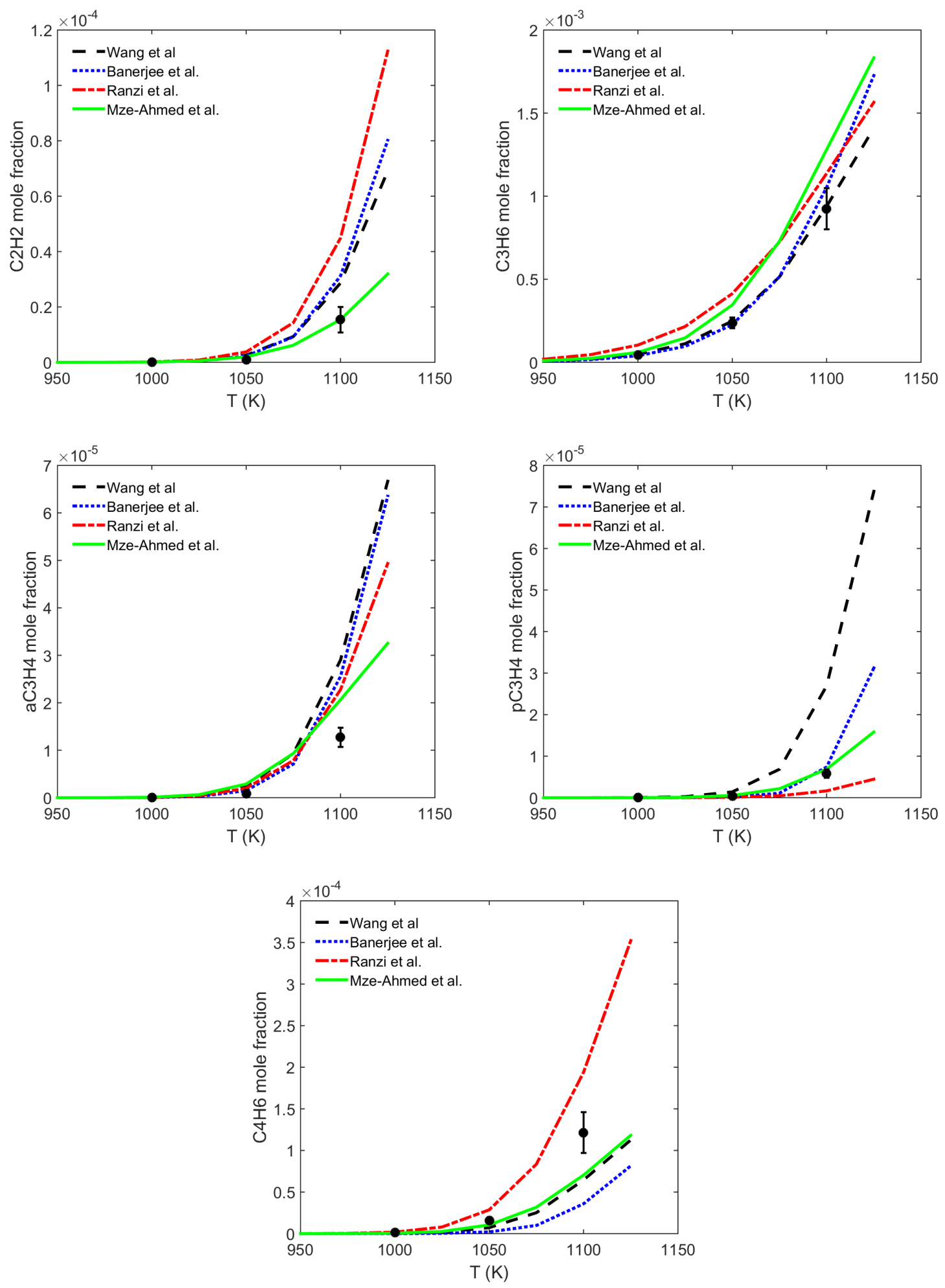

Figure 2.24: Species mole fraction as a function of temperature for $0.25 \%$ n-dodecane pyrolysis in the atmospheric pressure tube reactor at $10 \mathrm{~ms}$ residence time. Symbols are the experimental data with error bar indicating the uncertainty of the data. Lines are the model prediction using four different chemistry mechanisms. 

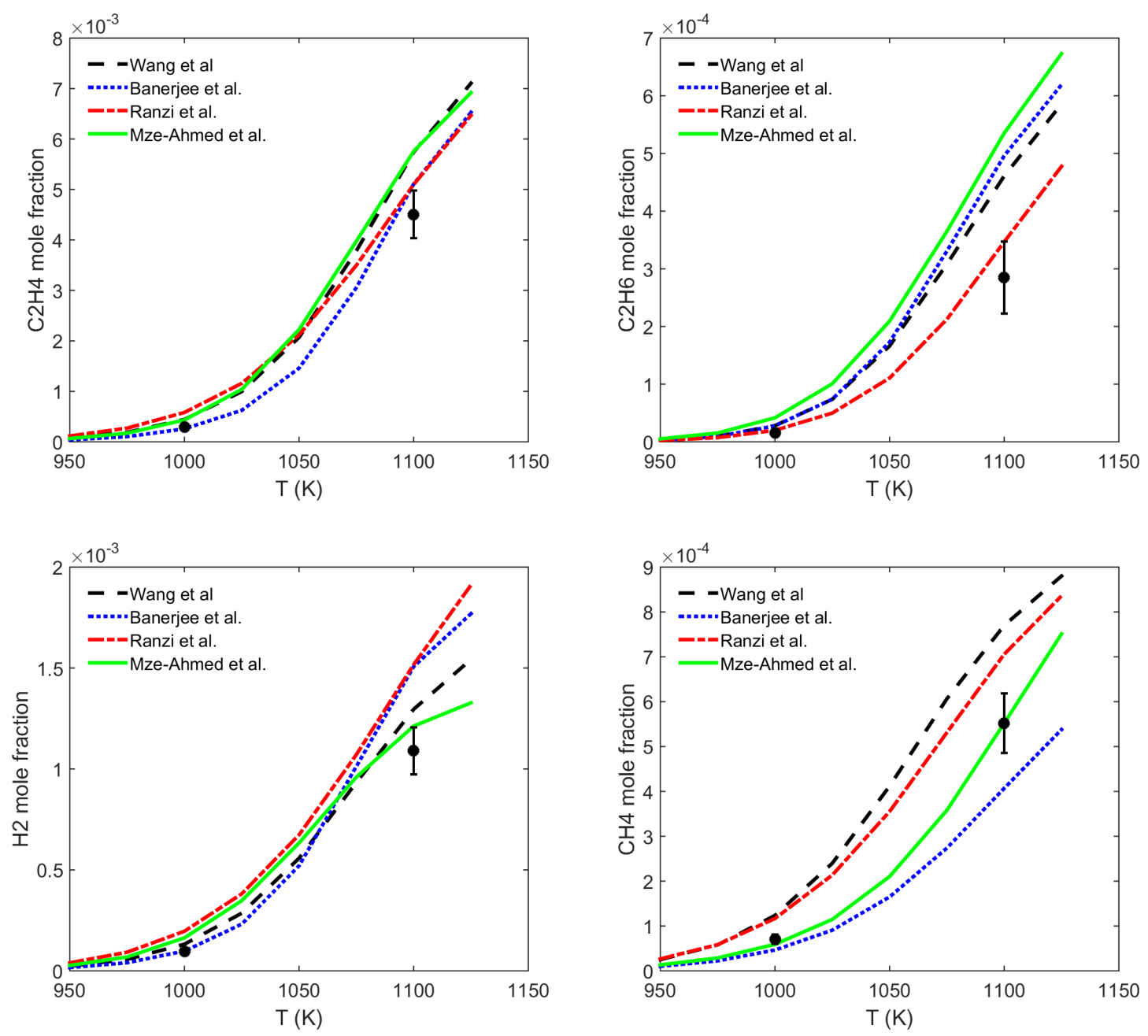

Figure 2.25: Species mole fraction as a function of temperature for $0.25 \%$ n-Dodecane pyrolysis in the atmospheric pressure tube reactor at $20 \mathrm{~ms}$ residence time. Symbols are the experimental data with error bar indicating the uncertainty of the data. Lines are the model prediction using four different chemistry mechanisms. 

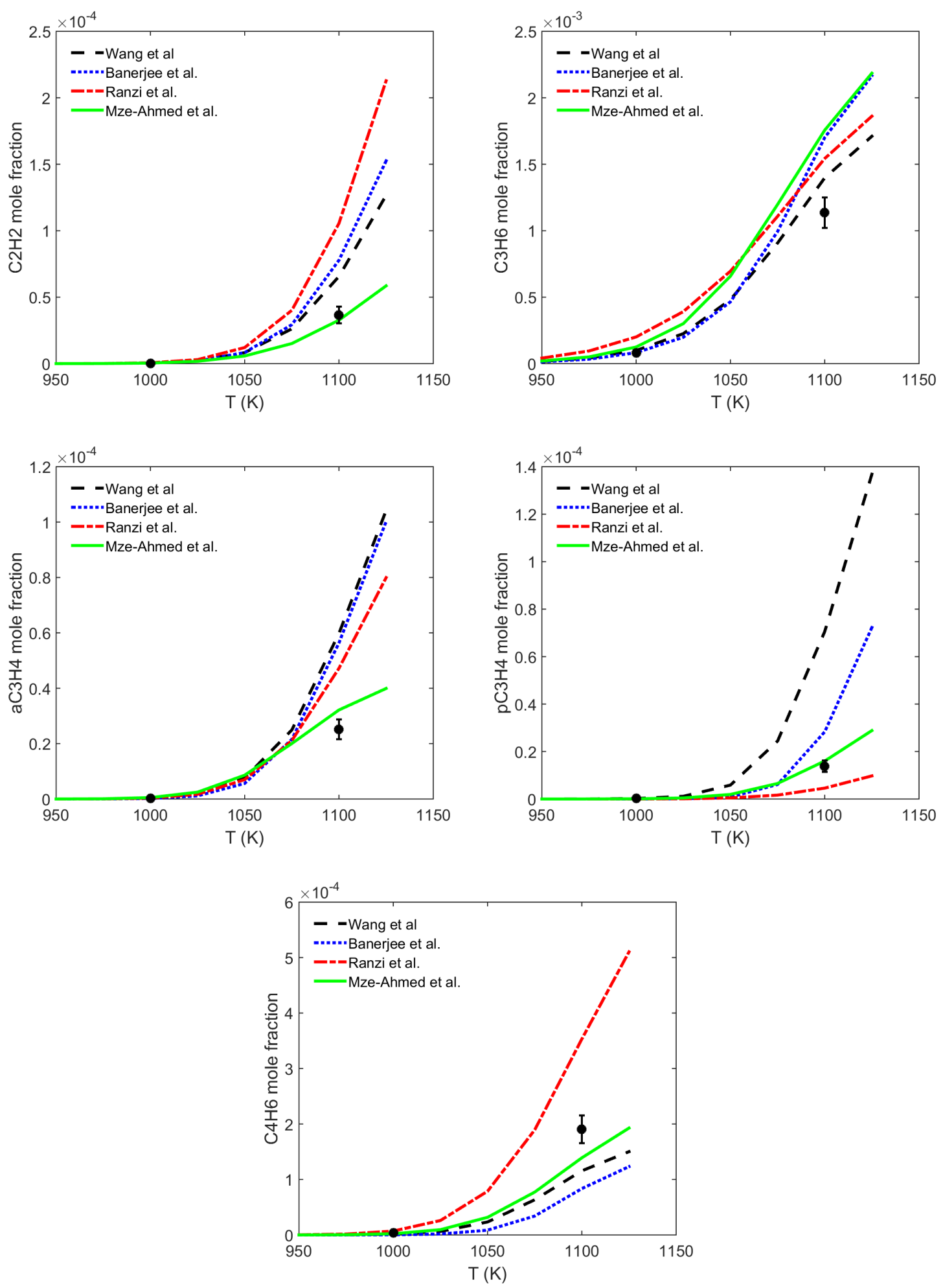

Figure 2.26: Species mole fraction as a function of temperature for $0.25 \%$ n-dodecane pyrolysis in the atmospheric pressure tube reactor at $20 \mathrm{~ms}$ residence time. Symbols are the experimental data with error bar indicating the uncertainty of the data. Lines are the model prediction using four different chemistry mechanisms. 

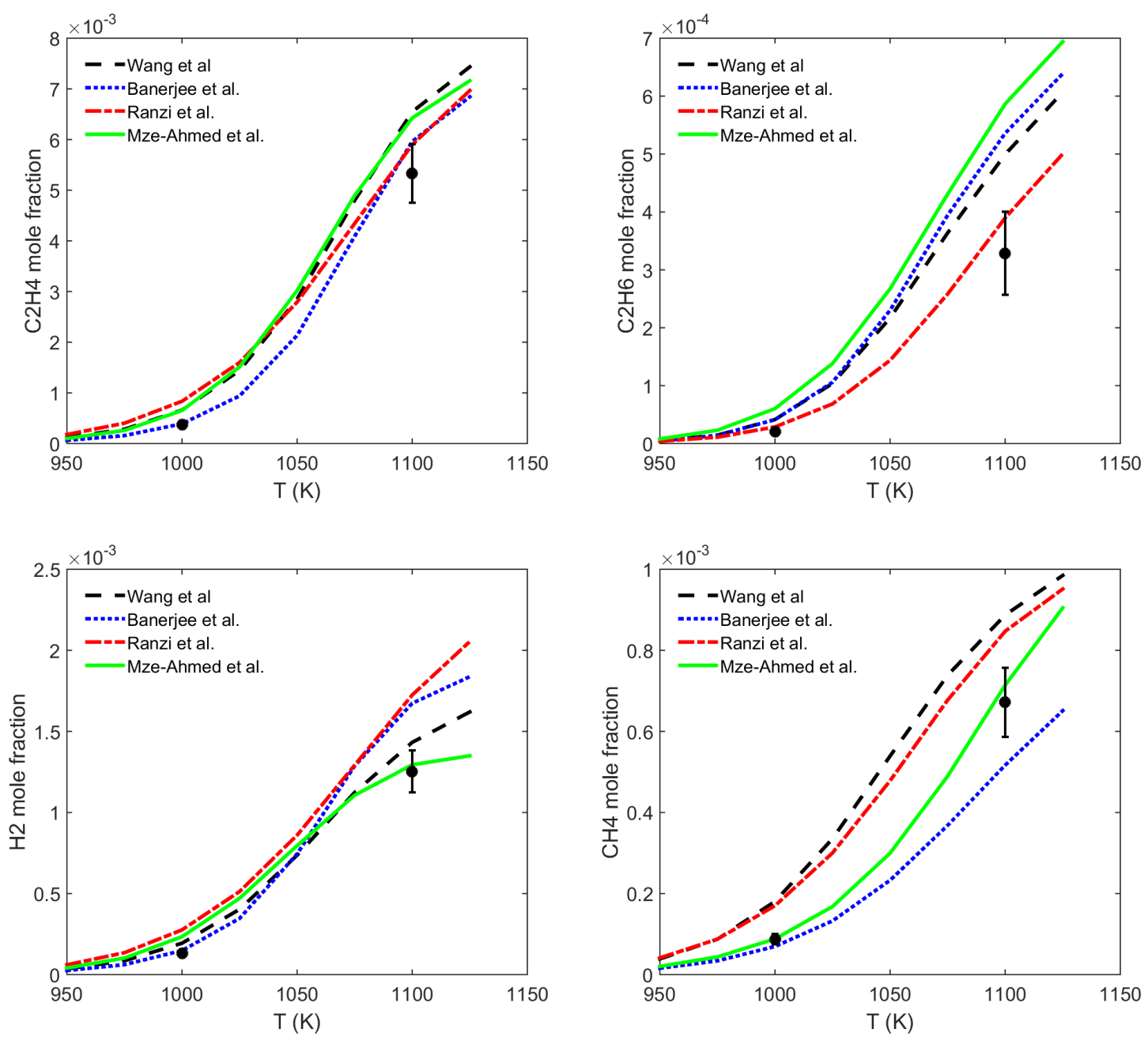

Figure 2.27: Species mole fraction as a function of temperature for $0.25 \%$ n-Dodecane pyrolysis in the atmospheric pressure tube reactor at $30 \mathrm{~ms}$ residence time. Symbols are the experimental data with error bar indicating the uncertainty of the data. Lines are the model prediction using four different chemistry mechanisms. 

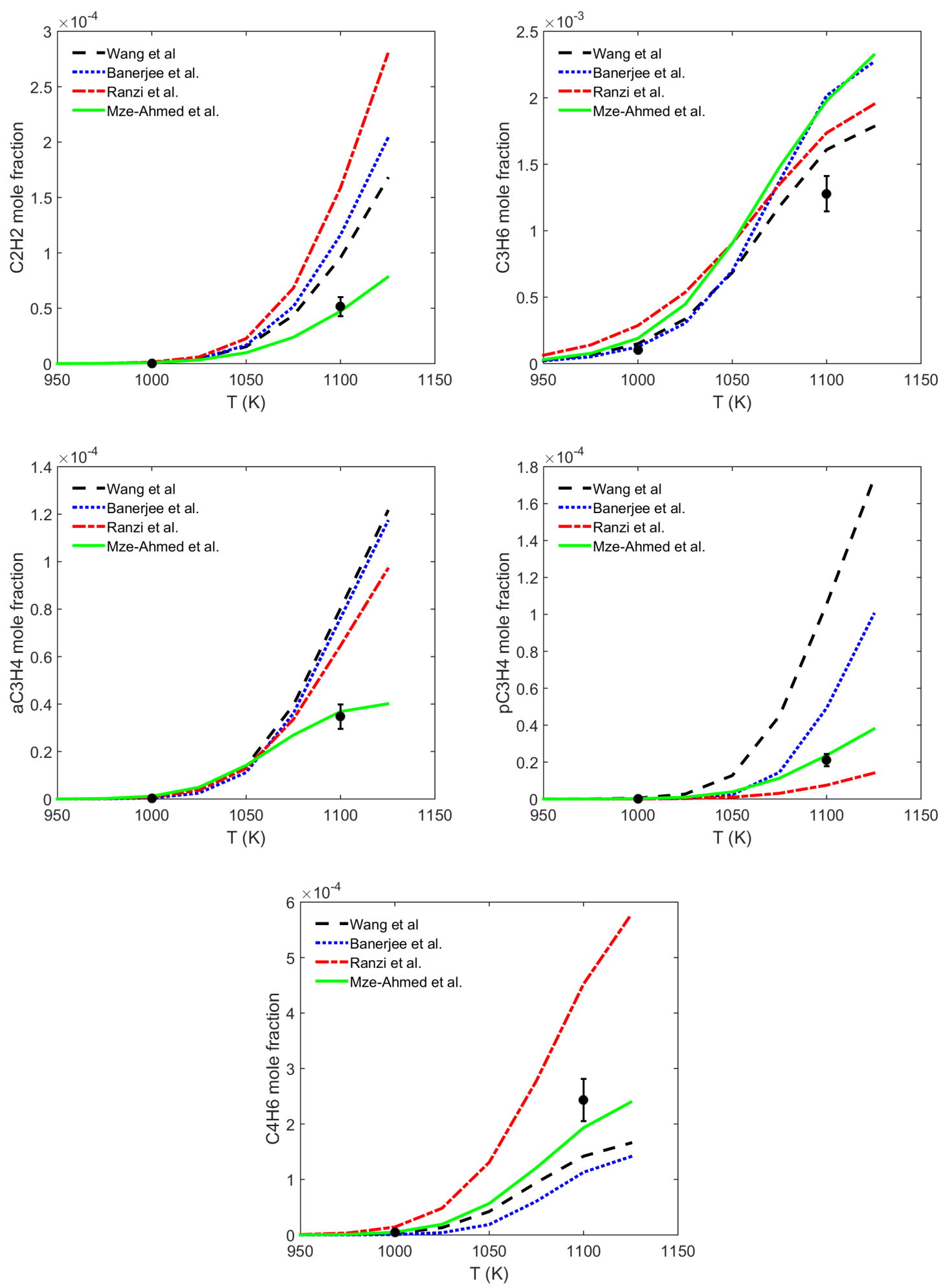

Figure 2.28: Species mole fraction as a function of temperature for $0.25 \%$ n-dodecane pyrolysis in the atmospheric pressure tube reactor at $30 \mathrm{~ms}$ residence time. Symbols are the experimental data with error bar indicating the uncertainty of the data. Lines are the model prediction using four different chemistry mechanisms. 

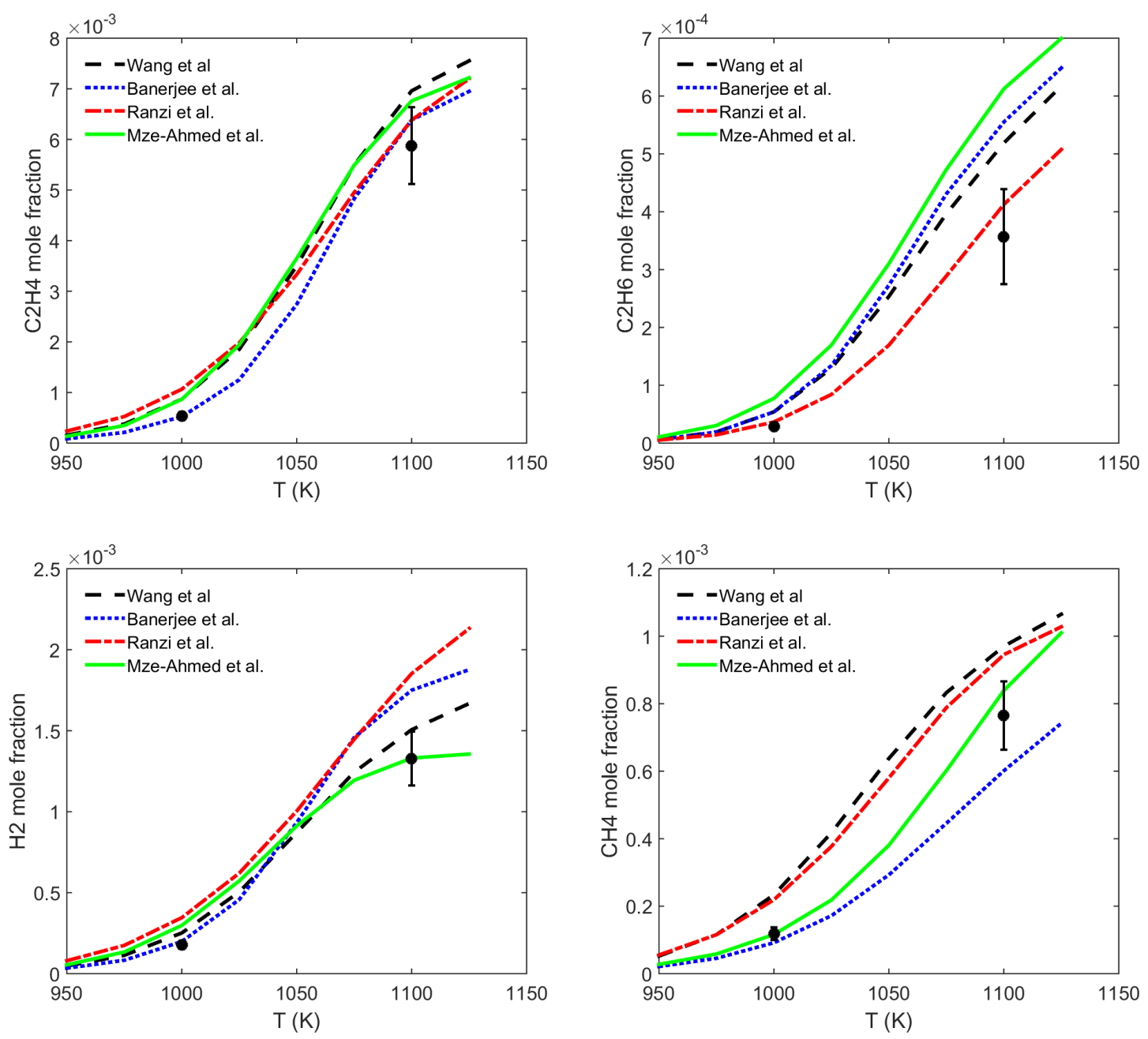

Figure 2.29: Species mole fraction as a function of temperature for $0.25 \%$ n-Dodecane pyrolysis in the atmospheric pressure tube reactor at $40 \mathrm{~ms}$ residence time. Symbols are the experimental data with error bar indicating the uncertainty of the data. Lines are the model prediction using four different chemistry mechanisms. 

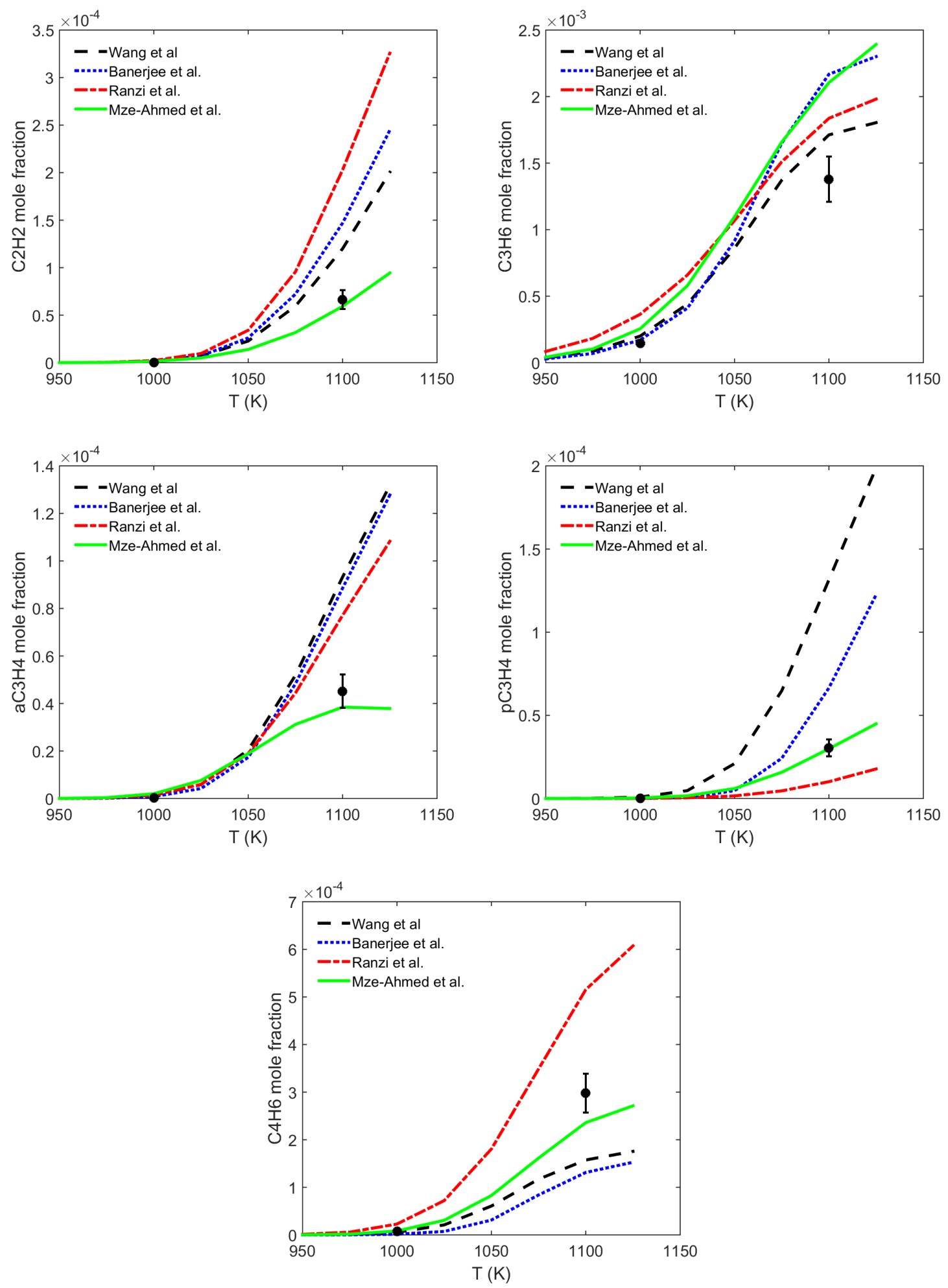

Figure 2.30: Species mole fraction as a function of temperature for $0.25 \%$ n-dodecane pyrolysis in the atmospheric pressure tube reactor at $40 \mathrm{~ms}$ residence time. Symbols are the experimental data with error bar indicating the uncertainty of the data. Lines are the model prediction using four different chemistry mechanisms. 

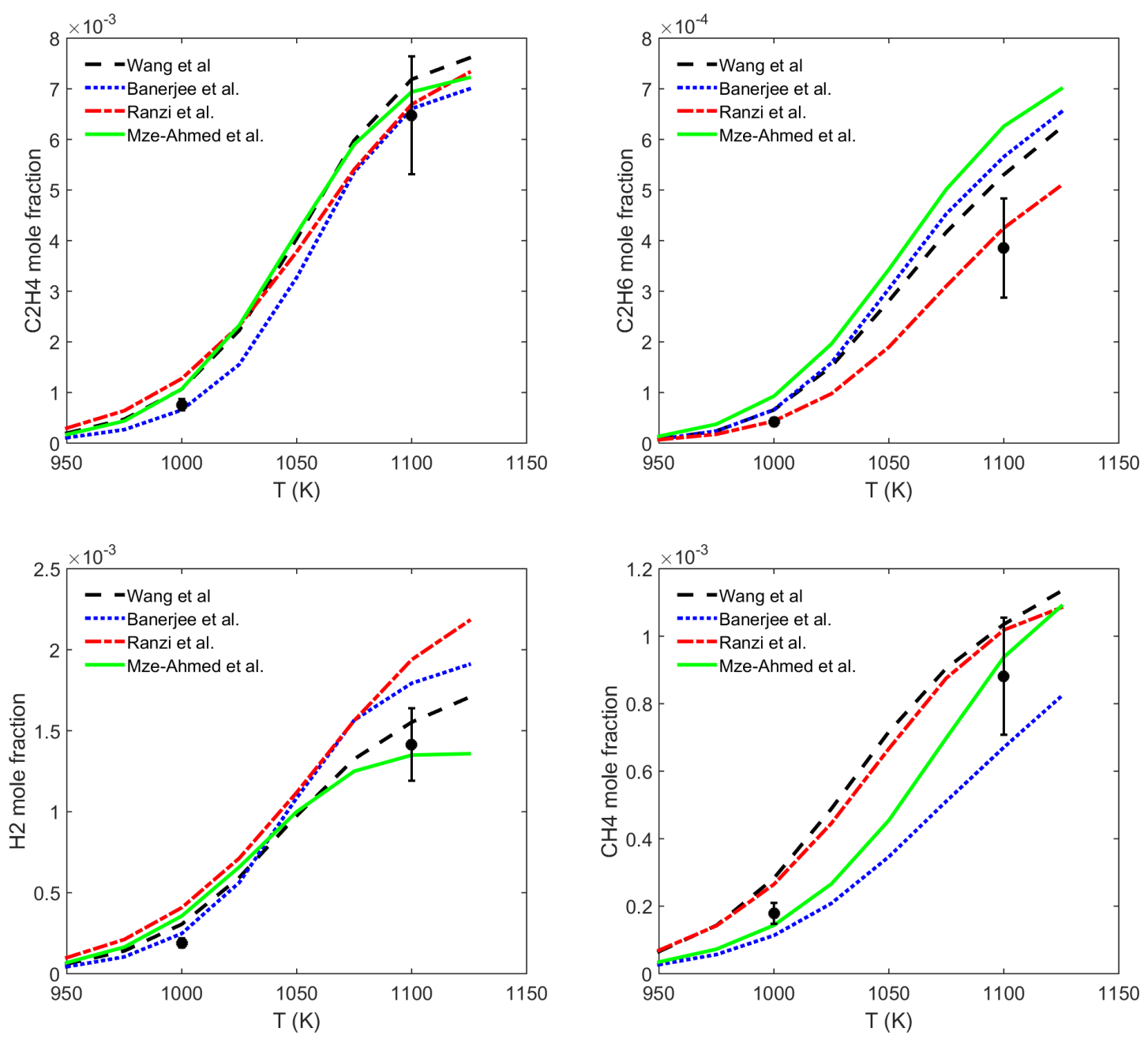

Figure 2.31: Species mole fraction as a function of temperature for $0.25 \%$ n-Dodecane pyrolysis in the atmospheric pressure tube reactor at $50 \mathrm{~ms}$ residence time. Symbols are the experimental data with error bar indicating the uncertainty of the data. Lines are the model prediction using four different chemistry mechanisms. 

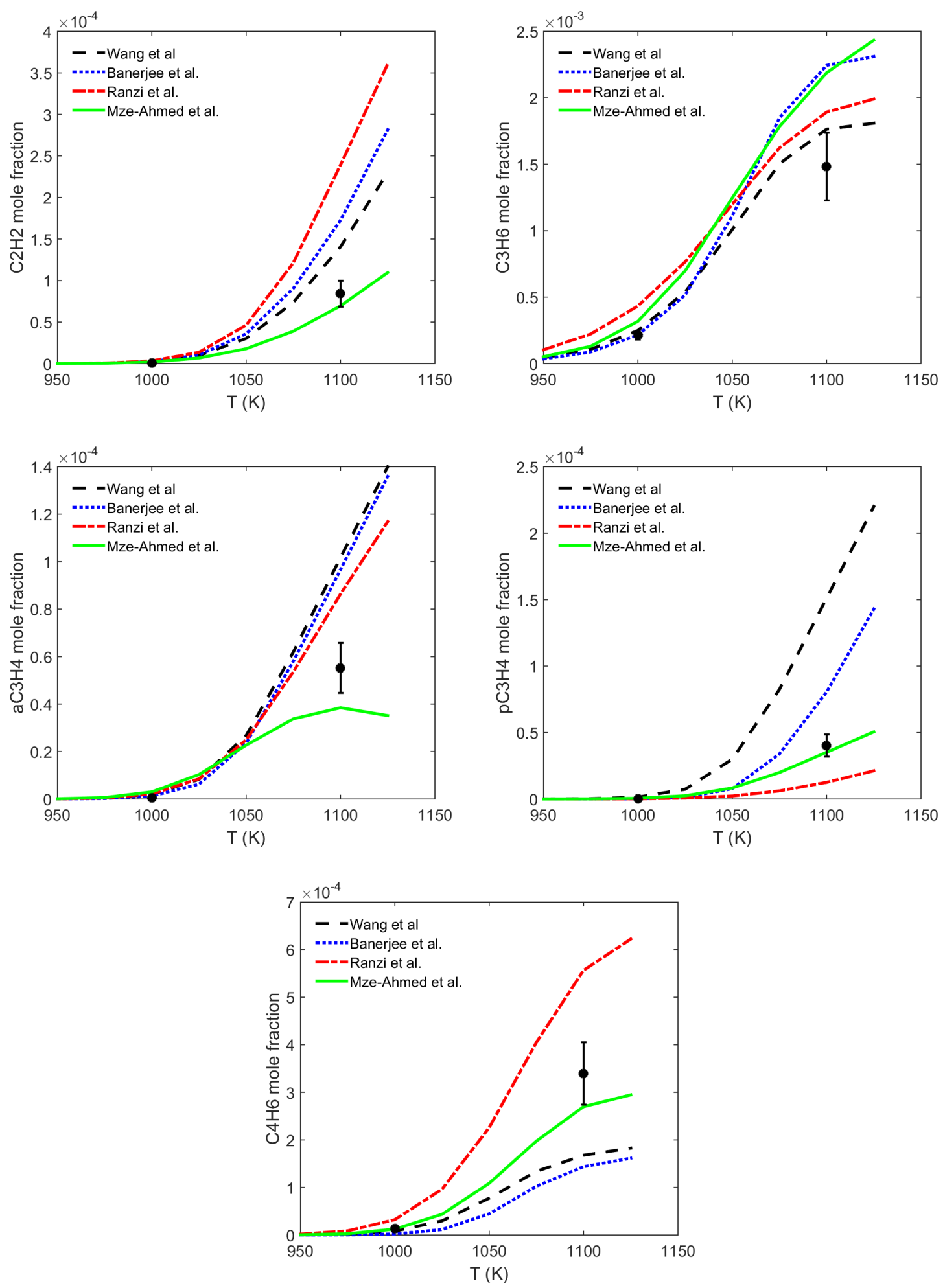

Figure 2.32: Species mole fraction as a function of temperature for $0.25 \%$ n-dodecane pyrolysis in the atmospheric pressure tube reactor at $50 \mathrm{~ms}$ residence time. Symbols are the experimental data with error bar indicating the uncertainty of the data. Lines are the model prediction using four different chemistry mechanisms. 

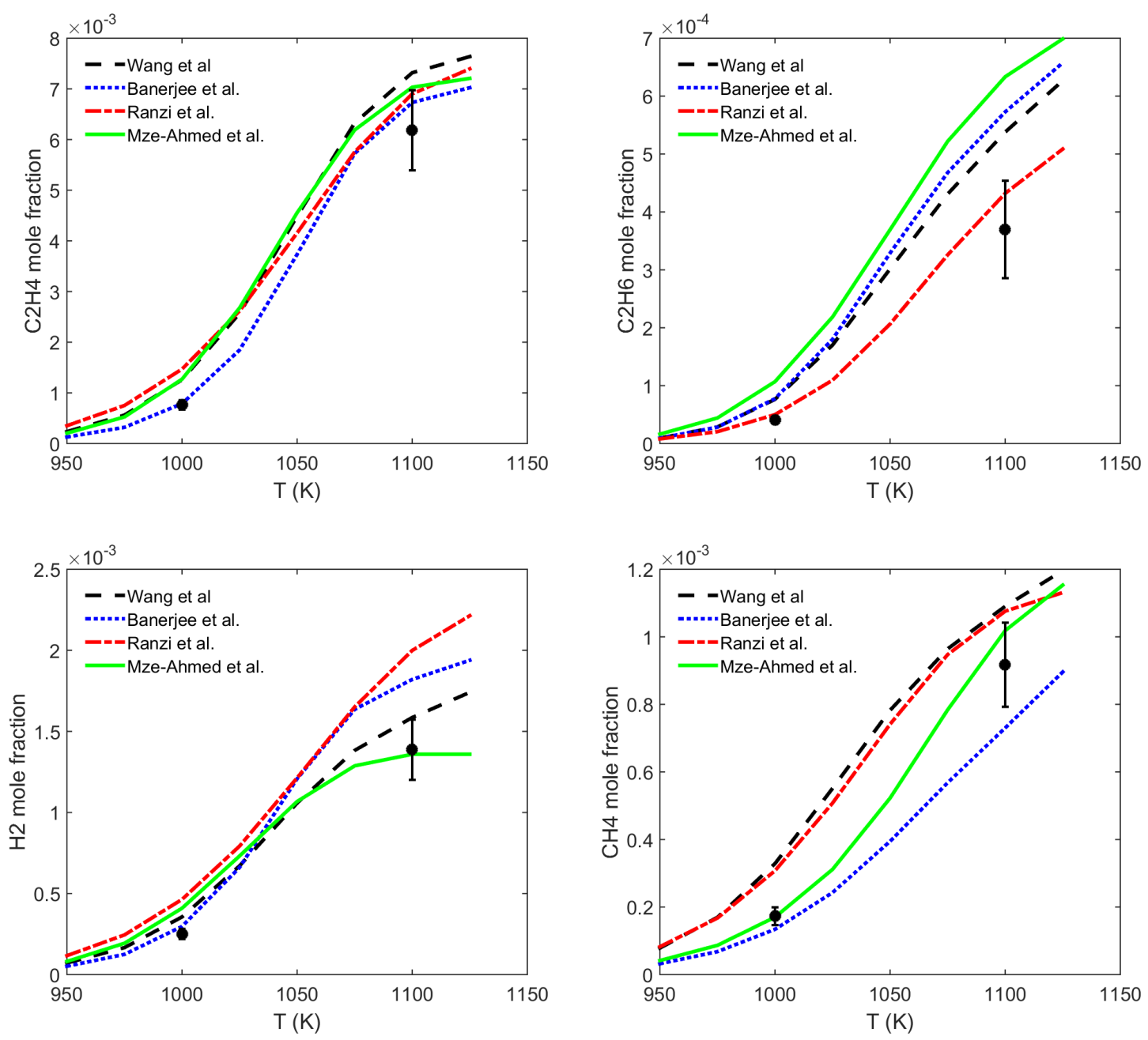

Figure 2.33: Species mole fraction as a function of temperature for $0.25 \%$ n-Dodecane pyrolysis in the atmospheric pressure tube reactor at $60 \mathrm{~ms}$ residence time. Symbols are the experimental data with error bar indicating the uncertainty of the data. Lines are the model prediction using four different chemistry mechanisms. 

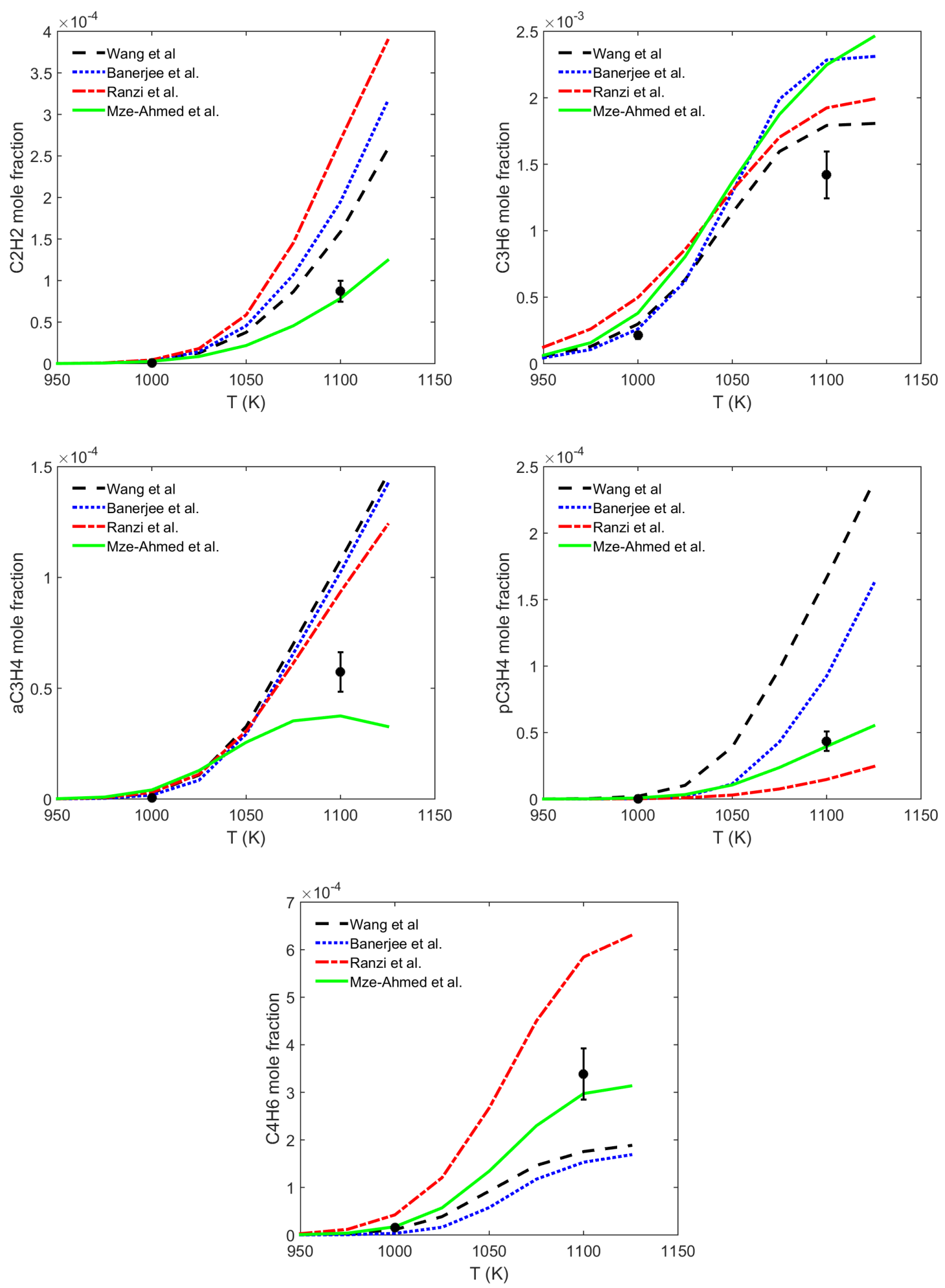

Figure 2.34: Species mole fraction as a function of temperature for $0.25 \%$ n-dodecane pyrolysis in the atmospheric pressure tube reactor at $60 \mathrm{~ms}$ residence time. Symbols are the experimental data with error bar indicating the uncertainty of the data. Lines are the model prediction using four different chemistry mechanisms. 


\subsection{JP-8 Pyrolysis Lumped Model}

Real aviation fuels such as JP-8 (POSF 10264) are a blend of different hydrocarbon components. Chemical kinetic models for these real jet fuel in order to accurately describe the pyrolysis and oxidation chemistry are developed by using surrogate fuel mixtures with large detailed reaction mechanisms in the combustion community $[77,78]$. Implementing these large detailed mechanisms in real reacting flow computations are not practical, not even with high-performance computational resources. For example, the recent surrogate reaction mechanism by Malewicki et al. [78] has 2080 species and 8310 reactions. These large number of parameters in the model does not necessarily mean the model predictions are accurate and robust. There are very limited experimental data available for the kinetic rate parameters of these elementary reactions, resulting in very poor estimation of most of the kinetic rates and reaction pathways.

In this study a very simple lumped chemical kinetic model is developed for the pyrolysis chemistry representation of JP-8. As demonstrated in recent studies, jet fuel mixtures quickly convert to a limited number of smaller hydrocarbons during high-temperature pyrolysis, which are mostly ethylene, methane, hydrogen, ethane, propene, isobutene, and benzene [77]. Therefore, the combustion process of these fuel can be represented by two stages. First the jet fuel endothermic cracking produces smaller hydrocarbons. Then, the exothermic oxidation of these smaller species occur in the second stage. This representation can be achieved by combining a simple lumped pyrolysis model for the jet fuel to describe the decomposition mechanism with a detailed $\mathrm{C} 0-\mathrm{C} 4$ sub-mechanism for the oxidation.

The experimental work and measurements of the endothermic pyrolysis of JP-8 were conducted by colleagues Shrestha and Simms. Based on the measured species a one-step lumped reaction model is suggested as 


$$
\begin{aligned}
\text { POSF } 10264 \rightarrow a C 2 H 4+b C 3 H 6 & +c H 2+d C H 4+e C 2 H 6 \\
& +f C 4 H 6+g i C 4 H 8+h C 2 H 2+i C 6 H 6
\end{aligned}
$$

In order to identify the stoichiometric coefficients in this reaction, the $\mathrm{C}$ and $\mathrm{H}$ atom conservation relations need to be satisfied. This requires knowing the exact molecular formula of JP-8 (POSF10264). The nominal molecular formula of C11H22 is assigned to the JP-8 fuel as reported in literature [79]. There are a total of 9 unknown parameters. Considering the two atom conservation relations $(\mathrm{H}$ and $\mathrm{C})$, there are 7 other relations required. The selectivity ratios of seven species with respect to ethylene are obtained from experiments. These ratios are calculated by the ratio of the mole fraction of selected species divided by ethylene mole fraction. By solving the 9 equations, all the 9 stoichiometric coefficients are calculated as

$$
\begin{gathered}
a=2.0780 \quad b=0.8312 \quad c=0.5922 \quad d=0.3408 \quad e=0.1621 \\
f=0.1662 \quad g=0.2909 \quad h=0.0208 \quad i=0.3025
\end{gathered}
$$

Since this lumped reaction is a simple one-step reaction with overall reaction order of unity, the reaction rate constant can be computed based on the concentration change as a function of time. The experimental data of the tube reactor at two temperatures of $1000 \mathrm{~K}$ and $1100 \mathrm{~K}$, provided the information to determine the reaction 
rate in a simple Arrhenius format of

$$
k=\operatorname{Aexp}\left(-\frac{E}{R T}\right)
$$

where $A$ is the pre-exponential factor , $E$ is the activation energy, $T$ is temperature, and $R$ is the universal gas constant.

Figure 2.35 shows the experimental data points and the fit line corresponding to the one-step JP-8 pyrolysis. The Arrhenius parameters are calculated by finding a linear fit line of log scale $k$ as a function of inverse temperature $1 / T$.

$$
\begin{gathered}
A=1.334 \times 10^{16} \\
E=71651 \quad \frac{\text { Cal }}{\text { mole }}
\end{gathered}
$$

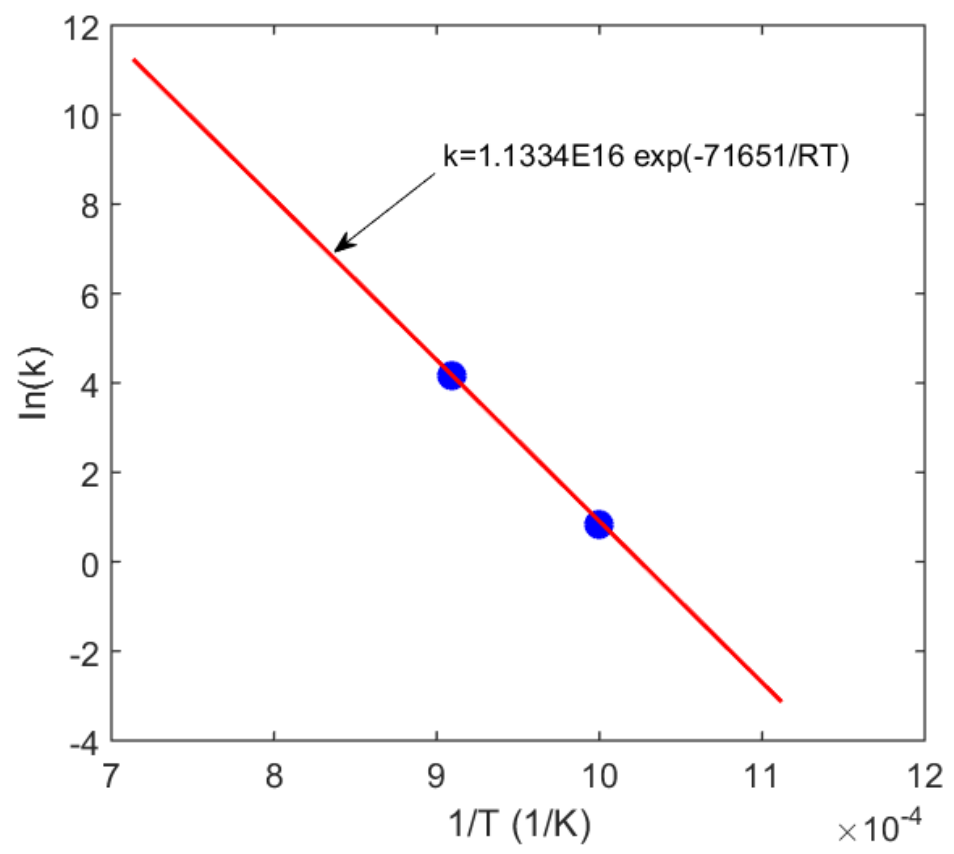

Figure 2.35: Reaction rate constant fit for the first order lumped reaction for JP-8 pyrolysis. Symbols show the experimental data from the UVa tube reactor. 
Figures 2.36 and 2.37 show the comparison of model prediction of species mole fraction profiles against the experimental data from the UVa tube reactor, for 1000 $\mathrm{K}$ and $1100 \mathrm{~K}$ operating temperature conditions, respectively. Symbols indicate the experimental data at two temperatures and $20 \mathrm{~ms}$ to $60 \mathrm{~ms}$ residence time variation. The blue solid line indicates the one-step lumped model developed in this study. The red dashed line indicates the prediction from a surrogate model by Dagaut and Cathonnet [80] which consists of 203 species and 1673 reactions. The green dash-dot line is a recent semi-global fast thermal pyrolysis model by Wang [81]. It includes six semi-global reactions describing the JP-8 pyrolysis along with a detailed C0-C4 species sub-mechanism [70].

The results comparison show that the one-step reaction model developed in this work has a better performance in predicting most of the important species compared to the other two models. However, The Dagaut and Cathonnet model performs better for predicting acetylene. 

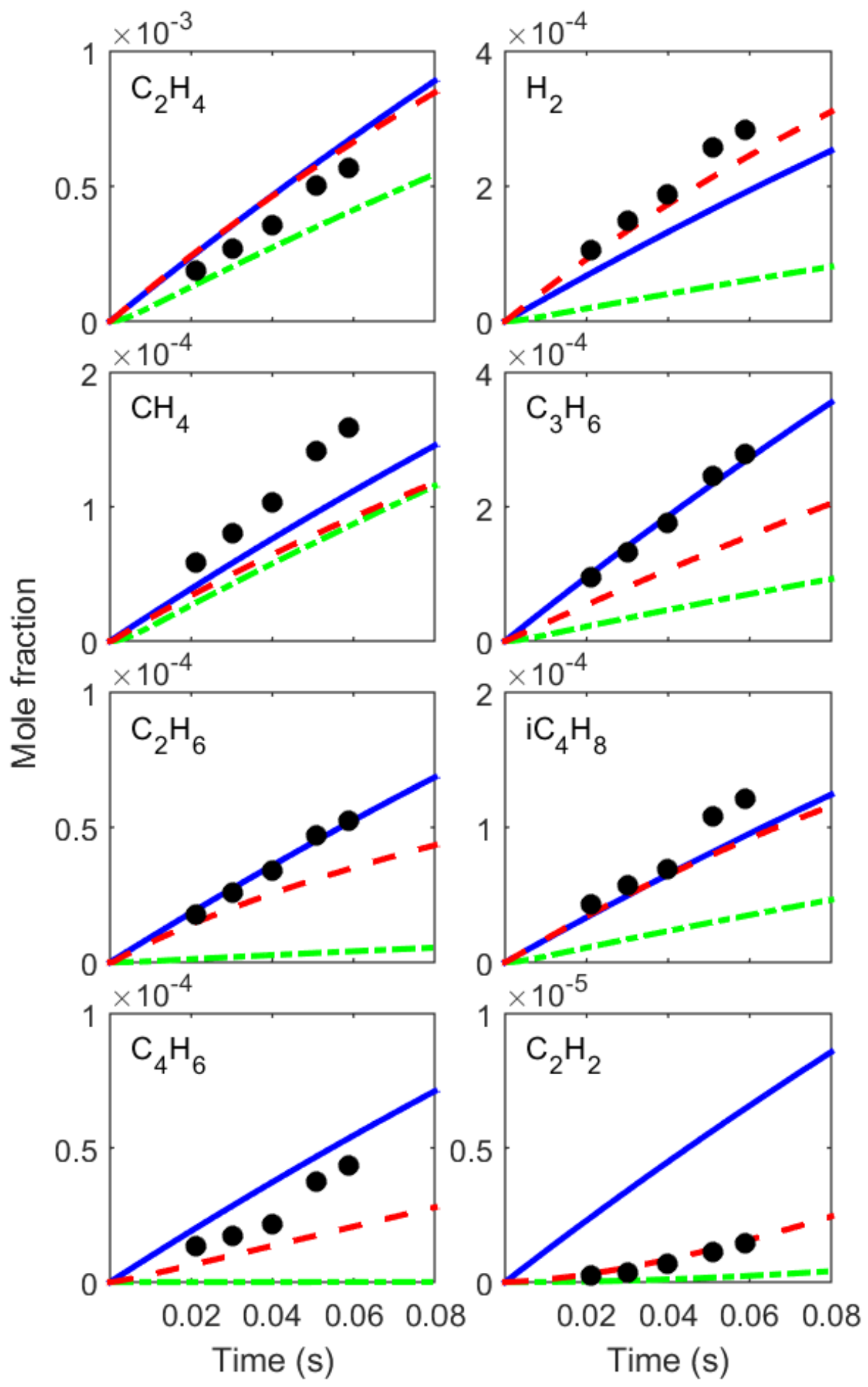

Figure 2.36: Comparison of model predictions of product species mole fraction in JP-8 pyrolysis data. Symbols are UVa tube reactor experiments, Solid blue line is the developed one-step model, dashed red line is Dagaut and Cathonnet model [80], dash-dot green line is Wang [81]. Tube reactor operating condition: $0.25 \%$ JP-8 fuel diluted in nitrogen, $\mathrm{p}=1 \mathrm{~atm}, \mathrm{~T}=1000 \mathrm{~K}$. 


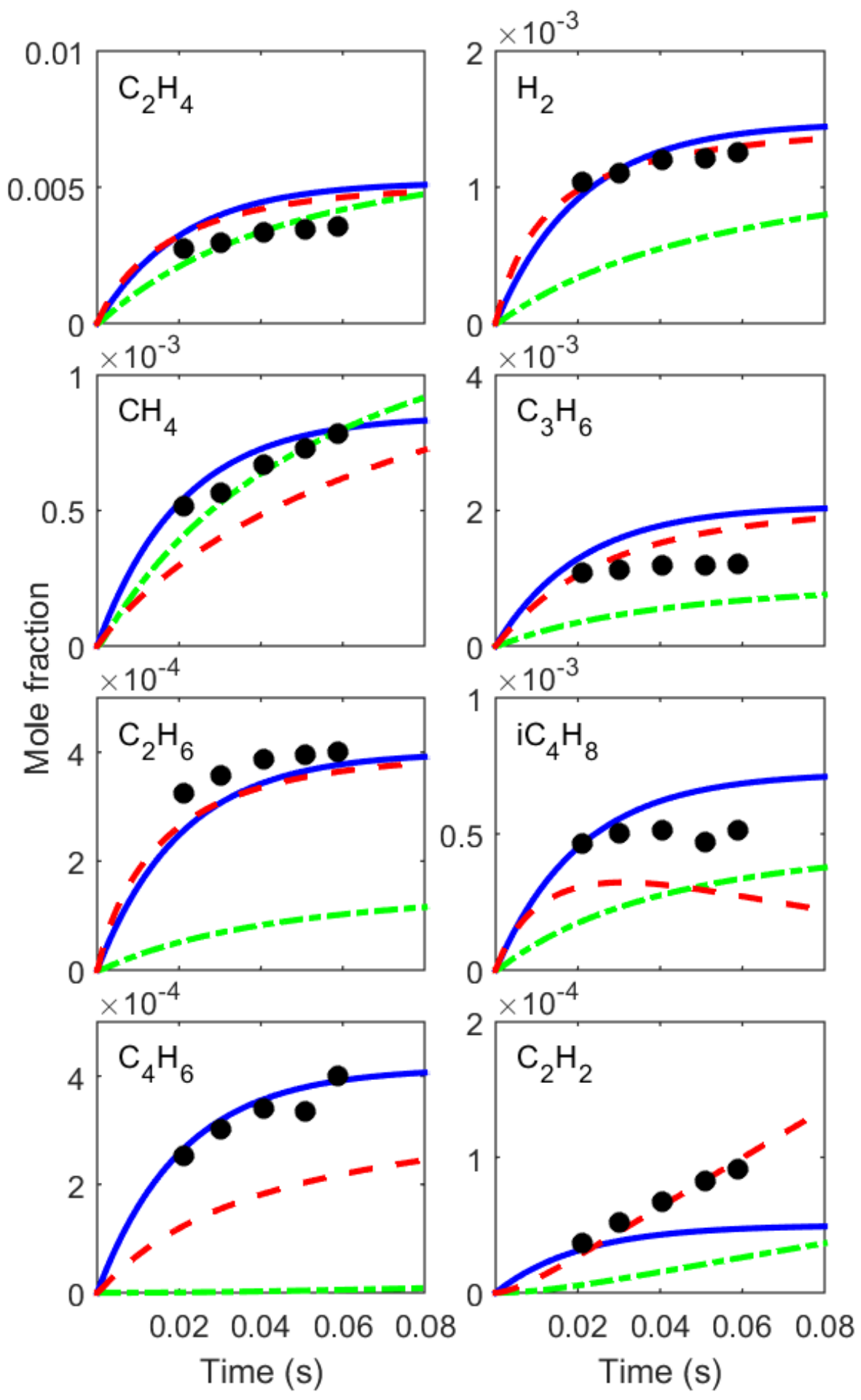

Figure 2.37: Comparison of model predictions of product species mole fraction in JP-8 pyrolysis data. Symbols are UVa tube reactor experiments, Solid blue line is the developed one-step model, dashed red line is Dagaut and Cathonnet model [80], dash-dot green line is Wang [81]. Tube reactor operating condition: $0.25 \%$ JP-8 fuel diluted in nitrogen, $\mathrm{p}=1 \mathrm{~atm}, \mathrm{~T}=1100 \mathrm{~K}$. 


\subsection{Summary}

Endothermic fuel pyrolysis in cooling channel was investigated based on a tubular microflow reactor developed at UVa Combustion and Energy Lab. The studied tube reactor is a representation of real cooling channels at hypersonic combustors.

First, ethane pyrolysis was considered as a test case to evaluate the flow reactor. In the analysis of ethane pyrolysis, three detailed chemical kinetic mechanisms were compared against the experimental data. The results for the major species were not very different between all the models. However, it is observed that the minor species mole fraction profiles are very different between the models. AramcoMech2.0 model showed better agreement with the experimental data for most of the measured species compared to other two models.

Before generalization to larger hydrocarbon molecules, n-butane was considered as another test fuel. In the analysis of n-butane pyrolysis, the predictions from three detailed chemical kinetic mechanisms were compared against the experimental data. All the three chemical kinetic models predicted most of the species profiles with large discrepancies. None of the models showed good agreement with the data for all of the measured species. This led to further investigations using sensitivity and uncertainty analysis in order to identify the target parameters that are most important to study more for improvement and optimization. The details of this analysis were presented in chapter 3.

Finally, as a large surrogate hydrocarbon molecule, the pyrolysis of n-dodecane was investigated in the flow reactor. For n-dodecane pyrolysis the predictions from four chemical kinetic models were compared with the experimental data. Mze-Ahmed et al. model predicted most of the major species with good agreement.

Also a simplified lumped one-step reaction model was developed for JP-8 pyrolysis. The results from this model and two other available detailed kinetic models were compared against the experimental data. The predictions from this developed simple 
model matched well with the experimental data for most of the major species. 


\section{Chapter 3}

\section{Chemical Kinetic Uncertainty Analysis of Endothermic Fuel Pyrolysis}

Chemical kinetic models are constantly being improved to better understand and predict the combustion processes, not only to describe accurately experimental data with simplified conditions but also be able to predict real life advanced reacting flow cases where experiments are difficult and expensive. However, models to represent such complex combustion processes are large detailed mechanism usually comprise of hundreds of species and thousands of reactions [82]. The thermo-kinetic parameters for these elementary reactions are derived from a wide variety of sources including direct measurements, global combustion experiments, and theoretical calculations.

Due to the large input parameters in detailed combustion models, the thermokinetic parameters have to be estimated in many cases rather than determined from direct kinetic experiments or theory calculations. Even there are significant uncertainties in the data used to parametrise combustion models. For example, hydrogen mechanisms that have been published in the recent years [35-39] which only have 
a small number of species and reactions and is considered one of the simplest wellknown chemistry models, there is still substantial disagreement between the chemical reaction rates. As a result, these input parameter uncertainties propagate through the combustion model leading to prediction uncertainties. A complete review of methods of sensitivity and uncertainty analyses in combustion chemistry modeling may be found in $[83,84]$. In order to improve the accuracy of the model prediction and its analysis capabilities, the input parameter uncertainties need to be reduced to the lowest possible level. However, due to the large number of parameters in the models, we need to prioritize the important effective parameters.

In order to identify the most important parameters to focus on improvement, there are methods which are used in this study: Sensitivity Analysis and Reaction Path Flux Analysis. These methods can help to determine the parameters that have the largest influence on combustion processes predictions and focus the improvement and optimization efforts on only those parameters.

\subsection{Sensitivity Analysis}

One of the important methods to understand how the solution of a chemical kinetic model is dependent on the model parameters (e.g. the reaction rate coefficients), is sensitivity analysis. To calculate the first-order local sensitivity, a brute-force approach is used here by computing the derivative of the model response (e.g. important species concentration, reactor temperature, or ignition delay time) with respect to the

model parameters. The brute-force normalized sensitivity coefficient $S_{k, i}$ of the model prediction solution $X_{k}$ with respect to the $i^{\text {th }}$ reaction rate parameter $A_{i}$ is calculated by

$$
S_{k, i}=\frac{A_{i}}{X_{k}} \frac{\partial X_{k}}{\partial A_{i}}
$$


where $X_{k}$ denotes the mole fraction of the $k^{t h}$ species whose sensitivity is being evaluated and $A_{i}$ denotes the pre-exponential factor of the $i^{t h}$ reaction rate.

For the studied case of n-dodecane pyrolysis at $1100 \mathrm{~K}$ and 1 atm condition, the sensitivity analysis was done at the residence time of $20 \mathrm{~ms}$ which corresponds to about half fuel consumption. The most sensitive reactions with respect to n-dodecane concentration as the target are shown in Figure 3.1 with their sensitivity coefficients for the JetSurF mechanism. Reactions with positive sensitivity coefficients in this figure, retard the rate of fuel decomposition while reactions with negative sensitivity coefficients accelerate the fuel decomposition. Here all types of reactions

$$
\mathrm{NC} 12 \mathrm{H} 26+\mathrm{H} \rightarrow \mathrm{C} 12 \mathrm{H} 25+\mathrm{H} 2
$$

with all various dodecyl isomers are among the most sensitive reactions and enhance the decomposition of $\mathrm{n}$-dodecane, while reaction

$$
\mathrm{CH} 3+\mathrm{CH} 3 \rightarrow \mathrm{C} 2 \mathrm{H} 6
$$

delays the decomposition process through the depletion of the methyl radical pool (chain terminating reaction). 


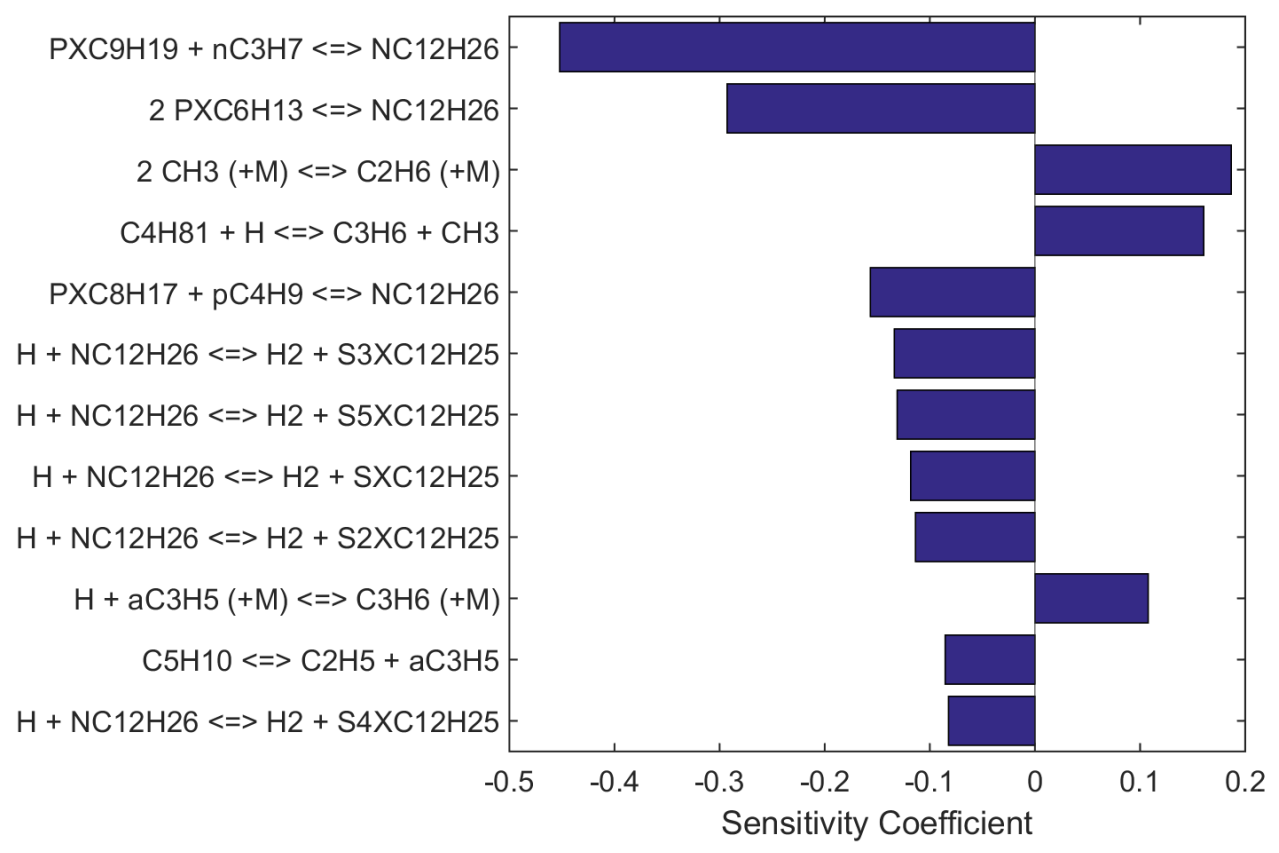

Figure 3.1: Largest sensitivities of n-dodecane decomposition to reaction rates for ndodecane pyrolysis in a flow reactor with atmospheric pressure, temperature of 1100 $\mathrm{K}$, and residence time of $20 \mathrm{~ms}$ using JetSurF model.

Sensitivity coefficients of the most important reactions with respect to ethylene concentration as the target are shown in Figure 3.2 for the JetSurF mechanism. Unlike the sensitivity of n-dodecane, reactions with positive sensitivity coefficients in this figure enhance the reactivity while reactions with negative sensitivity coefficients decrease the reactivity. 


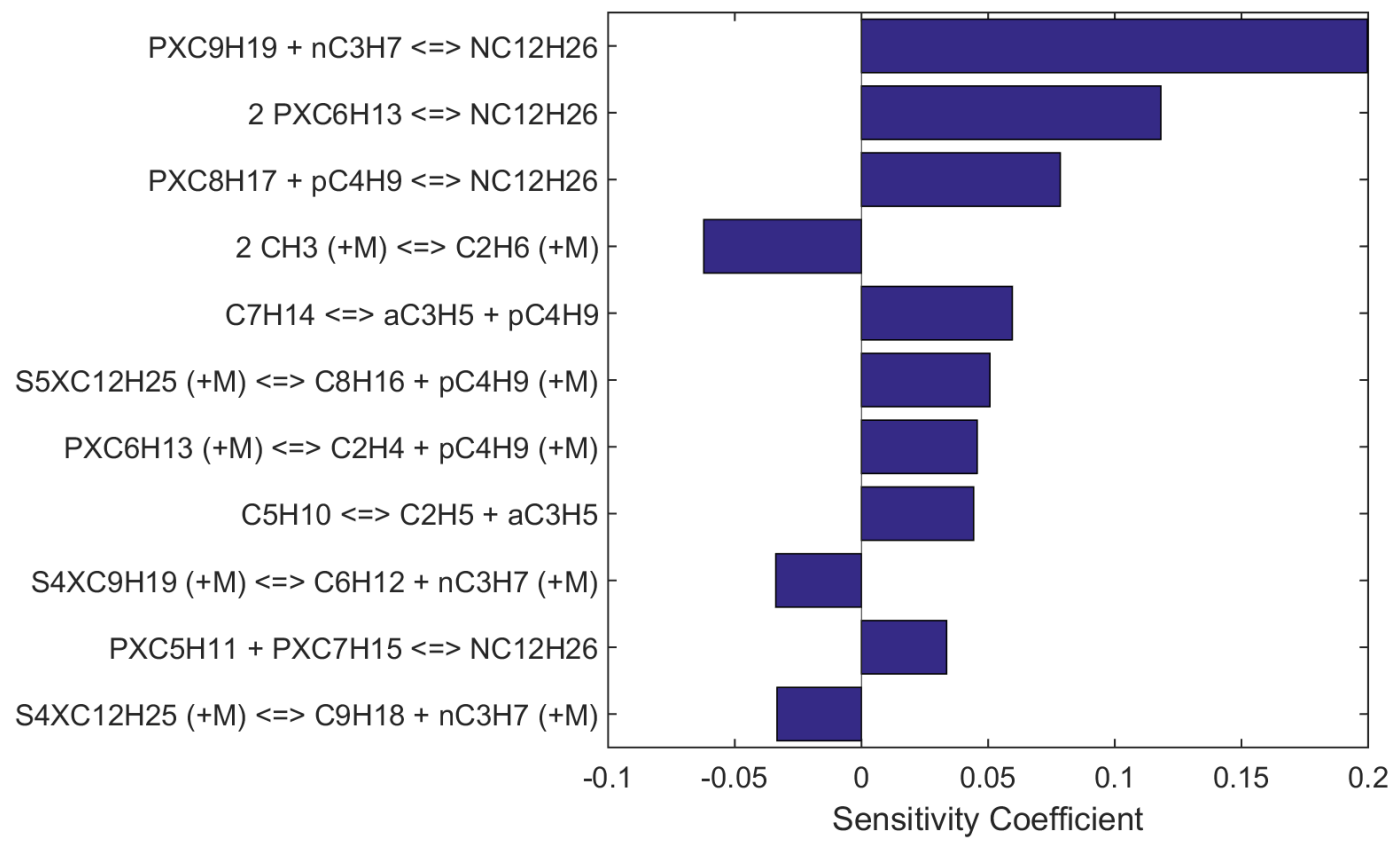

Figure 3.2: Normalized local sensitivity coefficients of ethylene production for the most important reactions in n-dodecane pyrolysis in a flow reactor with atmospheric pressure, temperature of $1100 \mathrm{~K}$, and residence time of $20 \mathrm{~ms}$ using JetSurF model.

\subsection{Reaction Pathway Analysis}

One of the ways to identify the important reactions and intermediate species associated with a chemical process and kinetic mechanism is to use the reaction pathway analysis. It is a useful way to visualize all the parameters and important reactions with their fluxes that affect the model solution. In this analysis, the rate of production flux of each species from all the reactions are calculated and stored at each time step. Based on this information the reaction path flux can be constructed with finding the largest fluxes and their ratios from one species to another.

Figures 3.3-3.6 are the path flux diagrams for n-dodecane pyrolysis at various residence times and $1 \mathrm{~atm}$ and $1100 \mathrm{~K}$ reacting condition. The thickness of the lines in these figures indicate the relative importance of the net flux from one species to another. At the very beginning of the fuel decomposition (residence time of about 4 
nanosecond), the two reactions

$$
N C 12 H 26 \rightarrow P X C 9 H 19+n C 3 H 7
$$

$$
N C 12 H 26 \rightarrow P X C 6 H 13
$$

are the dominant initiating reactions with $45 \%$ and $32 \%$ contribution, respectively (see Figure 3.3). The first reaction is the most important source of propyl radical formation which eventually is one of the main pathways to create ethylene. About $20 \%$ of the fuel decomposition flux is to create other alkyl fragments such as ethyl, butyl, heptyl, octyl, and decyl radicals.

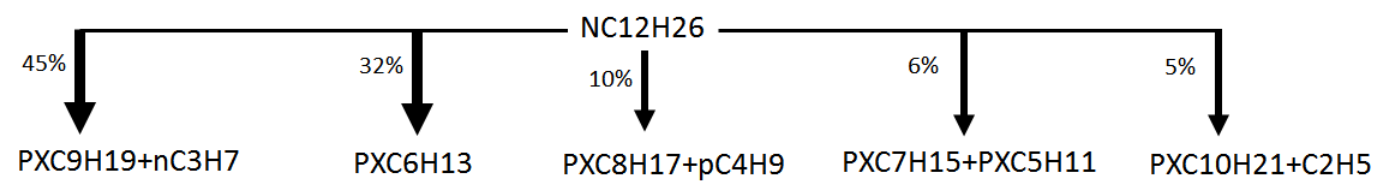

Figure 3.3: Reaction path flux analysis for $n$-dodecane pyrolysis at $\mathrm{p}=1$ atm, $\mathrm{T}=1100$ $\mathrm{K}$ and $4 \mathrm{~ns}$.

After some time where free radical pool has increased, the dominant decomposition path is changed from the $\mathrm{C}-\mathrm{C}$ bond fission to the hydrogen abstraction reactions forming dodecyl radical isomers. Figure 3.4 shows the path flux at $0.1 \mathrm{~ms}$ residence time. About $55 \%$ of the fuel decomposition flux is through the n-dodecane and hydrogen radical reactions to form dodecyl radical isomers and hydrogen molecule. Now only $26 \%$ of the flux is from to the two initiation reactions. Also less than $10 \%$ of the fuel decomposition flux is through the n-dodecane and methyl radical reactions (not shown in the figure) to form dodecyl radical isomers and methane molecule

$$
\text { NC12H26+CH3 } \rightarrow \text { C12H25+CH4. }
$$


In the next step, the different dodecyl radical isomers decay through multiple CC bond scission pathways to generate a range of alkyl radicals (mostly large C5-C9 species) and corresponding alkene species.

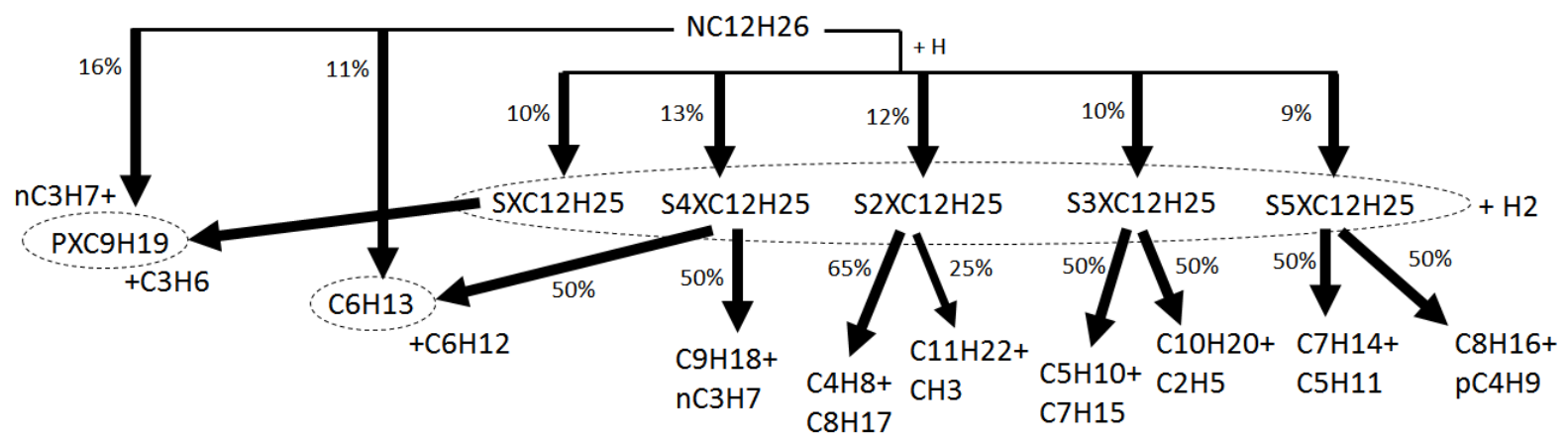

Figure 3.4: Reaction path flux analysis for n-dodecane pyrolysis at $\mathrm{p}=1 \mathrm{~atm}, \mathrm{~T}=1100$ $\mathrm{K}$ and $0.1 \mathrm{~ms}$.

At residence times long enough, the alkyl radicals produced from dodecyl and nonyl undergo more $\beta$ scission to create smaller alkenes and alkyl radicals. Figure 3.5 shows the path flux at $1 \mathrm{~ms}$ residence time. The decomposition fluxes for the relatively stable species (e.g. alkanes and alkenes) are omitted. The arrow thickness and the percentage assigned to each arrow, indicates qualitatively how the decomposition flux of each species is distributed. As can be seen, most of the larger alkyl radicals crack by $\beta$ scission to form ethyl, propyl, and butyl radicals which eventually are the primary source of ethylene formation. 


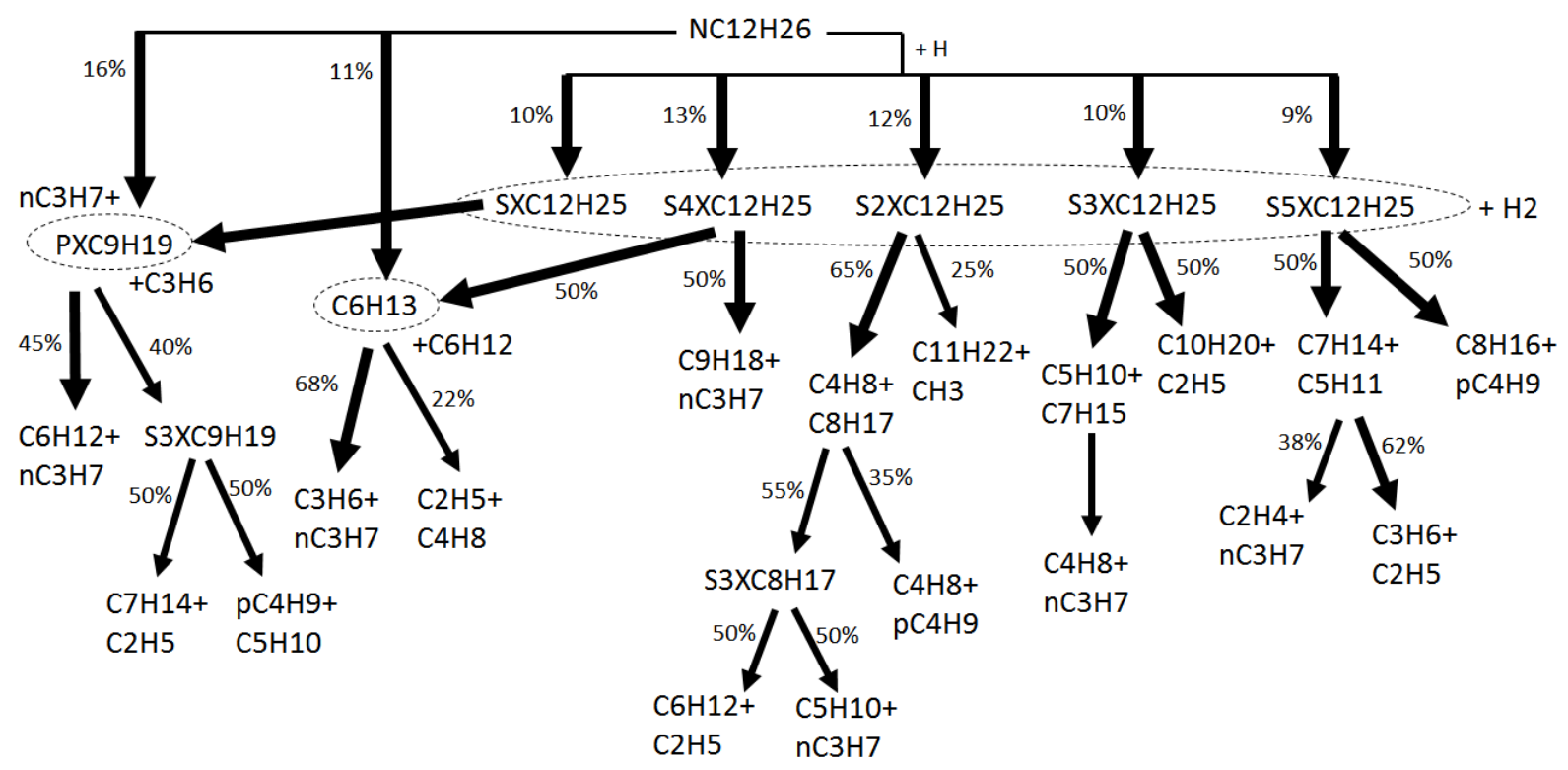

Figure 3.5: Reaction path flux analysis for n-dodecane pyrolysis at $\mathrm{p}=1 \mathrm{~atm}, \mathrm{~T}=1100$ $\mathrm{K}$ and $1 \mathrm{~ms}$.

Figure 3.6 shows the more downstream of the path flux analysis where the major stable species are generated. The three reactions

$$
\begin{gathered}
\mathrm{C} 2 \mathrm{H} 5 \rightarrow \mathrm{C} 2 \mathrm{H} 4+\mathrm{H} \\
n \mathrm{C} 3 \mathrm{H} 7 \rightarrow \mathrm{C} 2 \mathrm{H} 4+\mathrm{CH} 3 \\
p C 4 H 9 \rightarrow \mathrm{C} 2 \mathrm{H} 4+\mathrm{C} 2 \mathrm{H} 5
\end{gathered}
$$

contribute to ethylene formation by $37 \%, 34 \%$, and $15 \%$ at $10 \mathrm{~ms}$ residence time, respectively. It is worth mentioning that these three reactions do not appear in the most important reactions for ethylene sensitivity analysis (see Figure 3.2), which explains the importance of using reaction path flux analysis besides sensitivity analysis to determine the important chemical kinetic parameters. The methyl radical recom- 
bination reaction

$$
\mathrm{CH} 3+\mathrm{CH} 3 \rightarrow \mathrm{C} 2 \mathrm{H} 6
$$

is the primary source of ethane formation. 1-Butene is another major products of n-dodecane pyrolysis and the methyl and allyl reaction via

$$
\mathrm{CH} 3+a \mathrm{C} 3 \mathrm{H} 5 \rightarrow \mathrm{C} 4 \mathrm{H} 8
$$

is the major path flux for 1-butene formation. As seen in Figure 3.5, the primary path flux of hydrogen molecule is the hydrogen abstraction reactions from n-dodecane. Also methane major production flux is by methyl radical reactions. The three reaction involved to create methane are

$$
\mathrm{C} 2 \mathrm{H} 4+\mathrm{CH} 3 \rightarrow \mathrm{C} 2 \mathrm{H} 3+\mathrm{CH} 4
$$

$$
\mathrm{CH} 3+\mathrm{H} 2 \rightarrow \mathrm{CH} 4+\mathrm{H}
$$

$$
\mathrm{NC} 12 \mathrm{H} 26+\mathrm{CH} 3 \rightarrow \mathrm{C} 12 \mathrm{H} 25+\mathrm{CH} 4
$$

The last reaction includes five different dodecyl isomers in the model. 


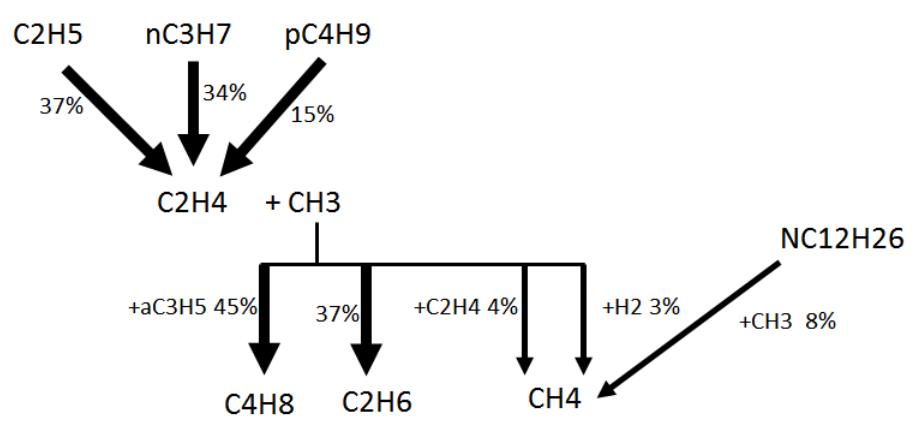

Figure 3.6: Reaction path flux analysis for $n$-dodecane pyrolysis at $\mathrm{p}=1 \mathrm{~atm}, \mathrm{~T}=1100$ $\mathrm{K}$ and $10 \mathrm{~ms}$.

\subsection{Model Uncertainty Analysis}

As stated in the beginning of the chapter, in order to improve the accuracy of the model predictions for combustion properties, it is necessary to determine the uncertainties associated with the model. The main goal of a kinetic model is to make predictions in cases where no experimental data exists. This prediction is only possible with confidence if uncertainties in the model are considered. For detailed reaction mechanisms with a large number of parameters, the input parameter space can be coupled and have high-dimensional correlation, making random sampling based methods more accurate effective to capture the global uncertainty properties. However, sampling based methods require a large number of samples for the convergence of the mean and variance of the result. Due to the limited computational resources, a sampling strategy must be selected to have the convergence with the minimum possible sample size.

There are different sampling methods available for uncertainty analysis. Monte Carlo analysis is one of the most common methods that generates a large number of independent random parameter sets to cover the uniform distribution of the input parameter space. Various sample generation approaches can be used for Monte Carlo 
simulation. Random sampling is the classical approach with the disadvantage of clustering of the samples in some regions of the input space and gaps in other regions, resulting in a poor convergence performance. A better approach is to use structured sampling with low discrepancy sequences which generate more uniformly distributed sample. An example of the low discrepancy sequences is the Sobol' quasi-random sequence [85]. Figure 3.7 shows a comparison of the two sampling methods for a two input parameter space where the sample size $N=1024$. The low discrepancy sequences provide a more uniform coverage compared to the random samples which lead to local clusters and gaps in some parts of the parameter space.

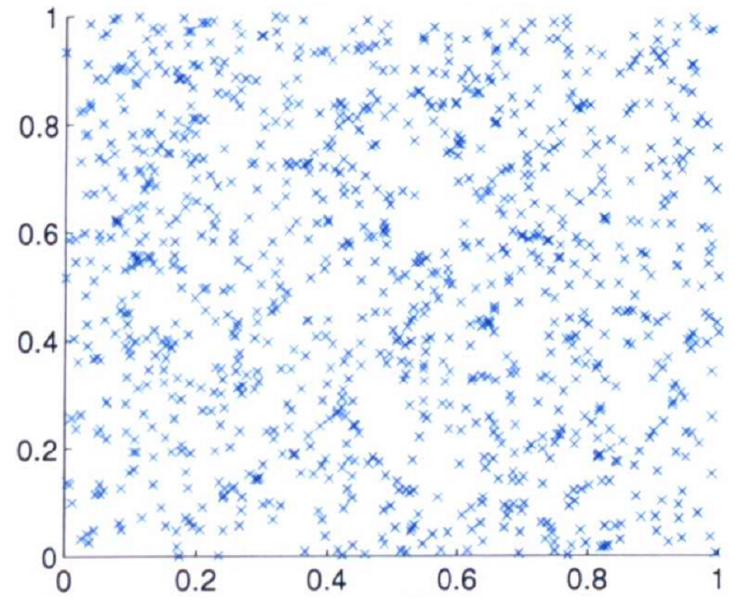

(a) Random sampling

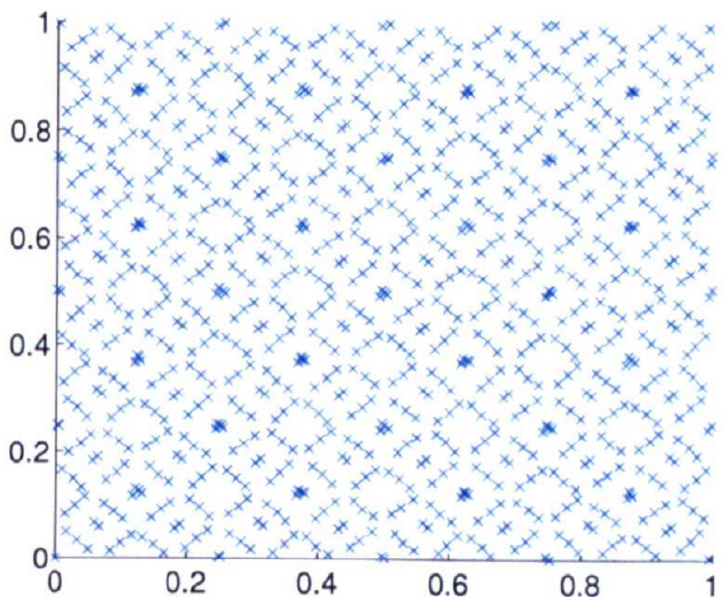

(b) Sobol' sequence

Figure 3.7: A comparison of sample distributions for two sampling methods in a twodimension parameter space with $\mathrm{N}=1024$ number of samples from [86]. (a) Random sampling and (b) quasi-random Sobol' sequence sample.

Various tests related to chemical kinetic model problems has been performed by Ziehn [86] and found that the convergence performance of the Sobol' quasi-random sequence to be much better than other sampling methods for the convergence properties. As a result, the Sobol' quasi-random sequence has been adapted for the Monte Carlo sampling method for this study.

In order to quantify the global uncertainty of the model, the input parameter uncertainty is needed. Here, the input parameter is the Arrhenius rate coefficient used 
for each elementary reaction. Reaction rate coefficients are reported by their most probable value as a function of temperature. The uncertainty of this rate coefficient is typically in the form of the uncertainty factor $f$ which is defined as:

$$
f=\frac{k^{\max }}{k^{0}}=\frac{k^{0}}{k^{\min }}
$$

where $k^{0}$ is the nominal value (most recommended and probable value) of the rate coefficient based on an assessment of available experimental and theoretical studies, and $k^{\min }$ and $k^{\max }$ are the extreme lower and upper bounds, respectively. The probability distribution of the uncertainty is assumed to be a uniform rectangular distribution. On a logarithmic scale the extreme values are positioned symmetrically around the nominal value. Baulch et al. $[87,88]$ has reported many uncertainty factor recommendations for a large number of reactions. As an example for uncertainty factor evaluation in literature, Figure 3.8 shows the Arrhenius plot for the rate coefficient of reaction $\mathrm{H}+\mathrm{CH} 4 \rightarrow \mathrm{CH} 3+\mathrm{H} 2$, evaluated by Baulch et al. [87], for which a relatively large number of experimental data are available. It is seen that the suggested $\mathrm{f}=2$ uncertainty bands cover most of the available experimental data. 


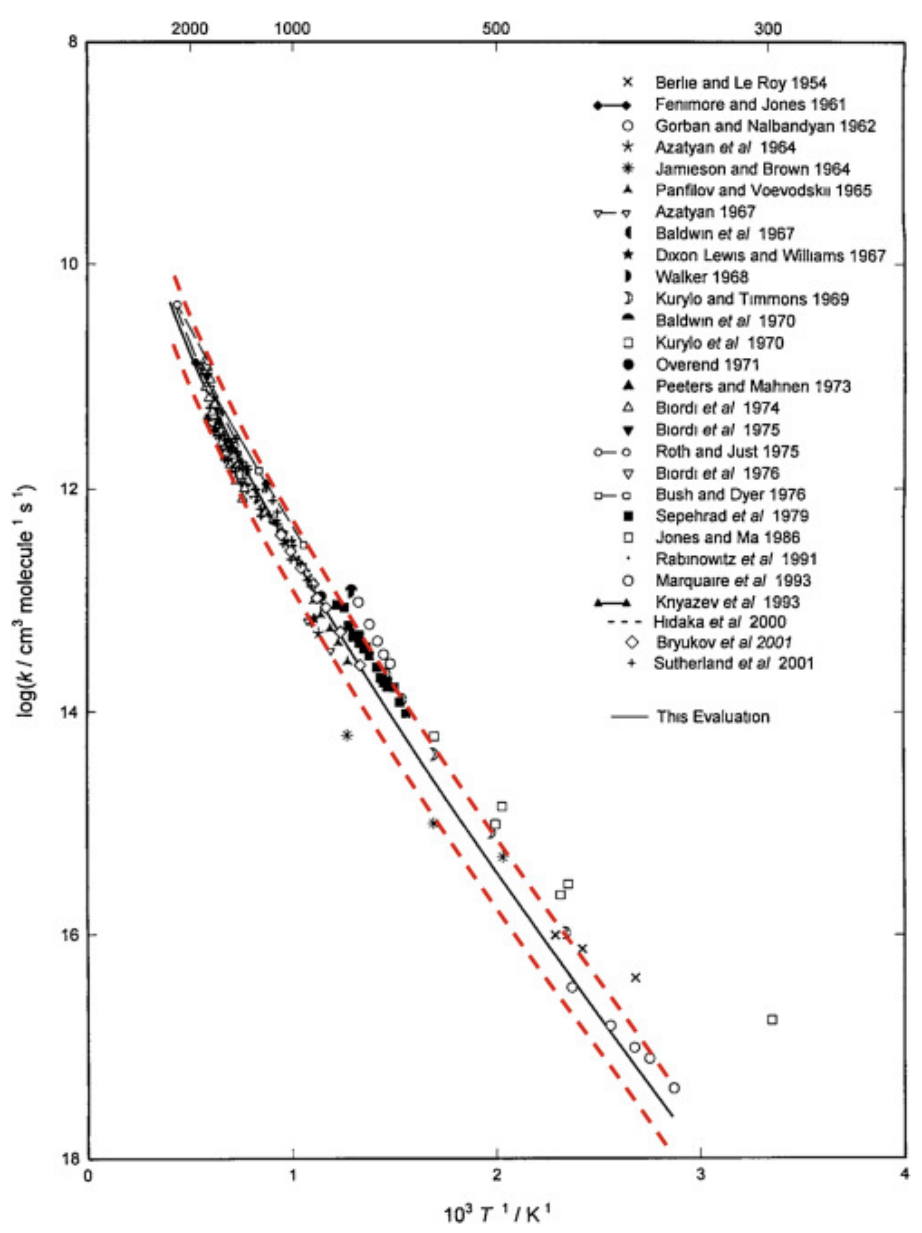

Figure 3.8: The reaction rate coefficient of $\mathrm{CH} 4+\mathrm{H} \rightarrow \mathrm{CH} 3+\mathrm{H} 2$ taken from literature (Baulch et al. [87]) . The solid line is the recommended reaction rate expression and the dashed lines indicate the upper and lower bounds according to the suggested uncertainty factor of 2 .

As mentioned previously, the sample size must be limited for avoid expensive computation. Therefore, only the most influential reaction rates within the model need to be used as the input parameter space. These important reactions are identified by using several criteria such as the sensitivity analysis of the important species (e.g. n-dodecane, ethylene, hydrogen, methane) and the path flux analysis. The selected reactions for the Monte Carlo simulation of n-dodecane pyrolysis (with flow reactor conditions of atmospheric pressure and temperature of $1100 \mathrm{~K}$ ) are listed in table 3.1 with their uncertainty factors. The uncertainty factor of the reactions are based on literature values reported in [87-89]. 
Table 3.1: Reactions selected for uncertainty propagation investigation for n-dodecane pyrolysis.

\begin{tabular}{|c|c|}
\hline Reaction & Uncertainty Factor \\
\hline PXC9H19+nC3H7=NC12H26 & 3 \\
\hline $\mathrm{PXC} 6 \mathrm{H} 13+\mathrm{PXC} 6 \mathrm{H} 13=\mathrm{NC} 12 \mathrm{H} 26$ & 3 \\
\hline $\mathrm{CH} 3+\mathrm{CH} 3(+\mathrm{M})=\mathrm{C} 2 \mathrm{H} 6(+\mathrm{M})$ & 2 \\
\hline $\mathrm{PXC} 8 \mathrm{H} 17+\mathrm{pC} 4 \mathrm{H} 9=\mathrm{NC} 12 \mathrm{H} 26$ & 3 \\
\hline $\mathrm{NC} 12 \mathrm{H} 26+\mathrm{H}=\mathrm{S} 3 \mathrm{XC} 12 \mathrm{H} 25+\mathrm{H} 2$ & 3 \\
\hline $\mathrm{NC} 12 \mathrm{H} 26+\mathrm{CH} 3=\mathrm{S} 3 \mathrm{XC} 12 \mathrm{H} 25+\mathrm{CH} 4$ & 3 \\
\hline $\mathrm{NC} 12 \mathrm{H} 26+\mathrm{CH} 3=\mathrm{S} 5 \mathrm{XC} 12 \mathrm{H} 25+\mathrm{CH} 4$ & 3 \\
\hline $\mathrm{C} 4 \mathrm{H} 81+\mathrm{H}=\mathrm{C} 3 \mathrm{H} 6+\mathrm{CH} 3$ & 5 \\
\hline $\mathrm{NC} 12 \mathrm{H} 26+\mathrm{H}=\mathrm{S} 5 \mathrm{XC} 12 \mathrm{H} 25+\mathrm{H} 2$ & 3 \\
\hline $\mathrm{NC} 12 \mathrm{H} 26+\mathrm{CH} 3=\mathrm{SXC} 12 \mathrm{H} 25+\mathrm{CH} 4$ & 3 \\
\hline $\mathrm{NC} 12 \mathrm{H} 26+\mathrm{CH} 3=\mathrm{S} 2 \mathrm{XC} 12 \mathrm{H} 25+\mathrm{CH} 4$ & 3 \\
\hline $\mathrm{NC} 12 \mathrm{H} 26+\mathrm{H}=\mathrm{SXC} 12 \mathrm{H} 25+\mathrm{H} 2$ & 3 \\
\hline $\mathrm{aC} 3 \mathrm{H} 5+\mathrm{H}(+\mathrm{M})=\mathrm{C} 3 \mathrm{H} 6(+\mathrm{M})$ & 3 \\
\hline $\mathrm{C} 3 \mathrm{H} 6+\mathrm{H}=\mathrm{aC} 3 \mathrm{H} 5+\mathrm{H} 2$ & 2 \\
\hline $\mathrm{C} 5 \mathrm{H} 10=\mathrm{C} 2 \mathrm{H} 5+\mathrm{aC} 3 \mathrm{H} 5$ & 3 \\
\hline $\mathrm{C} 2 \mathrm{H} 4+\mathrm{CH} 3=\mathrm{C} 2 \mathrm{H} 3+\mathrm{CH} 4$ & 5 \\
\hline $\mathrm{C} 2 \mathrm{H} 3(+\mathrm{M})=\mathrm{C} 2 \mathrm{H} 2+\mathrm{H}(+\mathrm{M})$ & 1.5 \\
\hline $\mathrm{PXC} 6 \mathrm{H} 13(+\mathrm{M})=\mathrm{C} 2 \mathrm{H} 4+\mathrm{pC} 4 \mathrm{H} 9(+\mathrm{M})$ & 3 \\
\hline $\mathrm{C} 7 \mathrm{H} 14=\mathrm{pC} 4 \mathrm{H} 9+\mathrm{aC} 3 \mathrm{H} 5$ & 3 \\
\hline $\mathrm{C} 4 \mathrm{H} 81+\mathrm{H}=\mathrm{C} 2 \mathrm{H} 4+\mathrm{C} 2 \mathrm{H} 5$ & 3 \\
\hline
\end{tabular}

To perform the Monte Carlo simulation, the rate coefficients of all the reactions from the Table 3.1 were perturbed by the values generated from the Sobol' sequence and normalized by the uncertainty factor. The simulation needed to run for a each case from the total number of samples. The convergence of the Monte Carlo simulation was achieved in this study by 3000 number of samples. The UVA high performance computing cluster was used in order to run the Monte Carlo simulations with total run time exceeding 50000 CPU-hours.

Figures 3.9-3.13 show the species mole fraction profile as a function of time for the explored tube reactor experiment of n-dodecane pyrolysis in at $1100 \mathrm{~K}$ and atmospheric pressure. Symbols indicate the UVA reactor experimental measurement with the error bars indicate the uncertainty of the data. Lines are the four model (Wang et al. [69], banerjee et al. [19], Ranzi et al. [75], Mze-Ahmed et al. [76]) predictions 
with their nominal reaction rate values. The shaded area indicates the $2 \sigma$ uncertainty of the Wang et al. model prediction. 

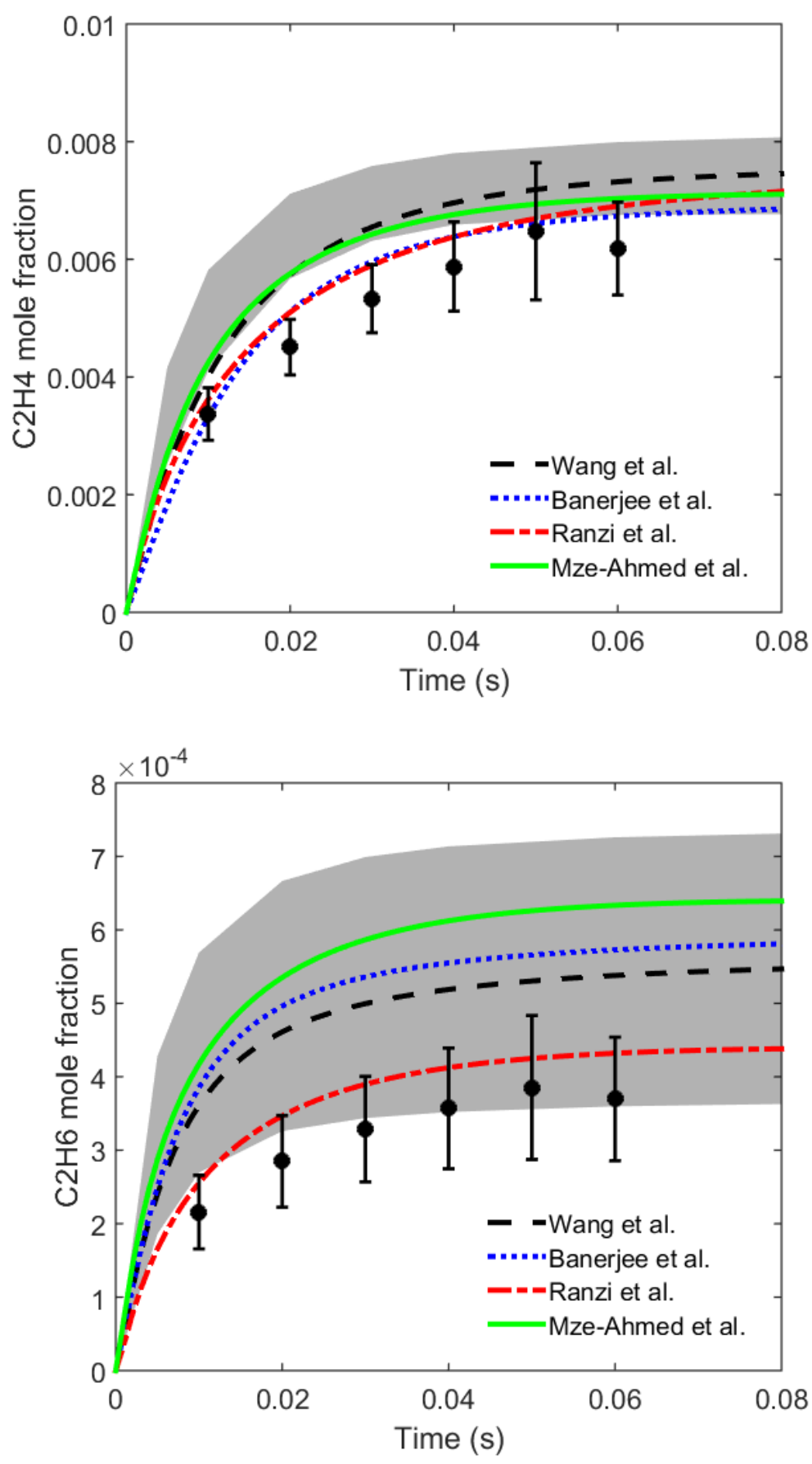

Figure 3.9: Species mole fraction profile as a function of time for n-dodecane pyrolysis in a $1100 \mathrm{~K}$ constant temperature and atmospheric pressure tube reactor. Symbols are UVa reactor experimental data with the error bars indicate uncertainty of the data and lines are model predictions with nominal rates. The shaded area indicates the $2 \sigma$ model prediction uncertainty. 

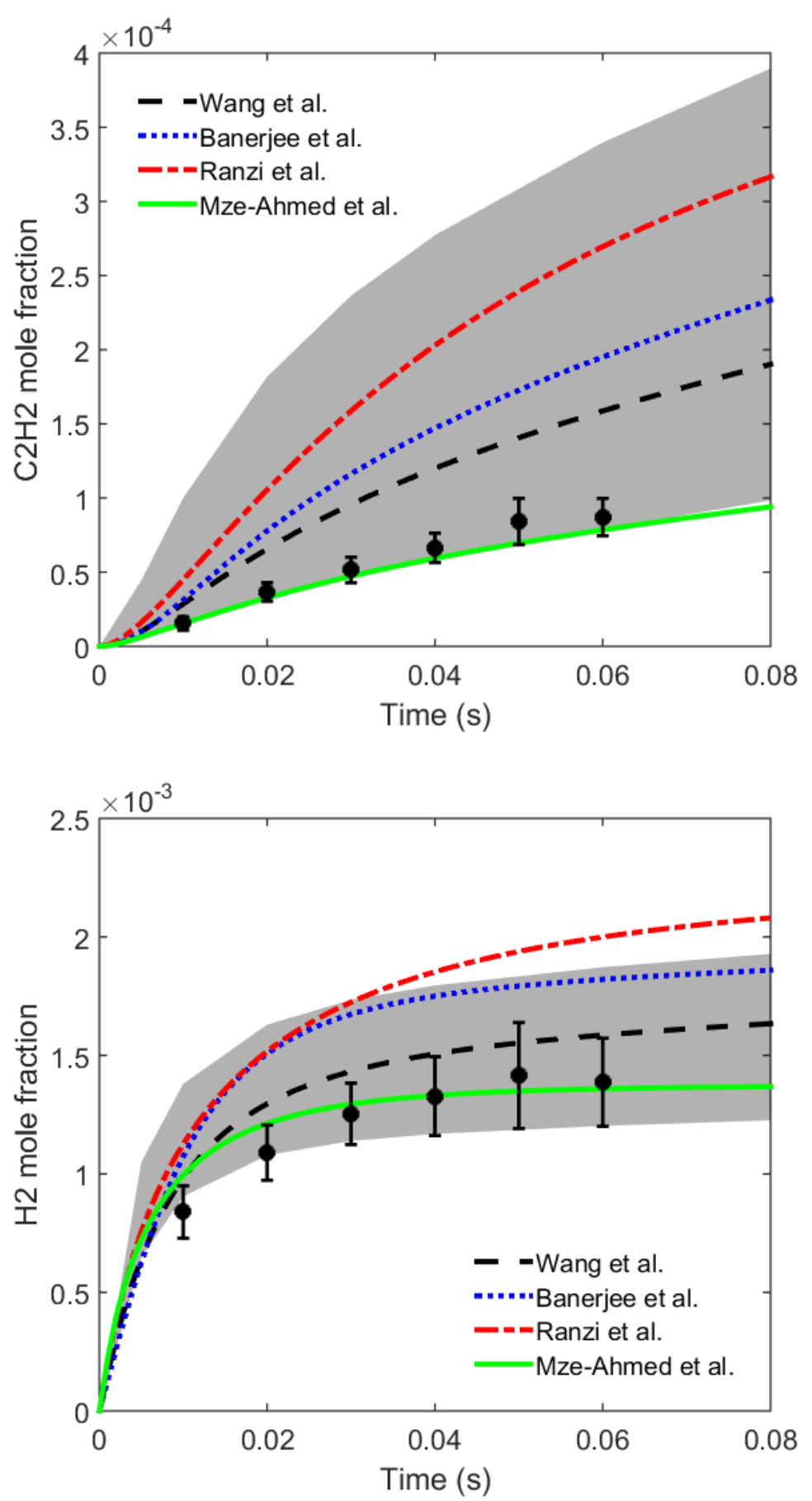

Figure 3.10: Species mole fraction profile as a function of time for n-dodecane pyrolysis in a $1100 \mathrm{~K}$ constant temperature and atmospheric pressure tube reactor. Symbols are UVa reactor experimental data with the error bars indicate uncertainty of the data and lines are model predictions with nominal rates. The shaded area indicates the $2 \sigma$ model prediction uncertainty. 

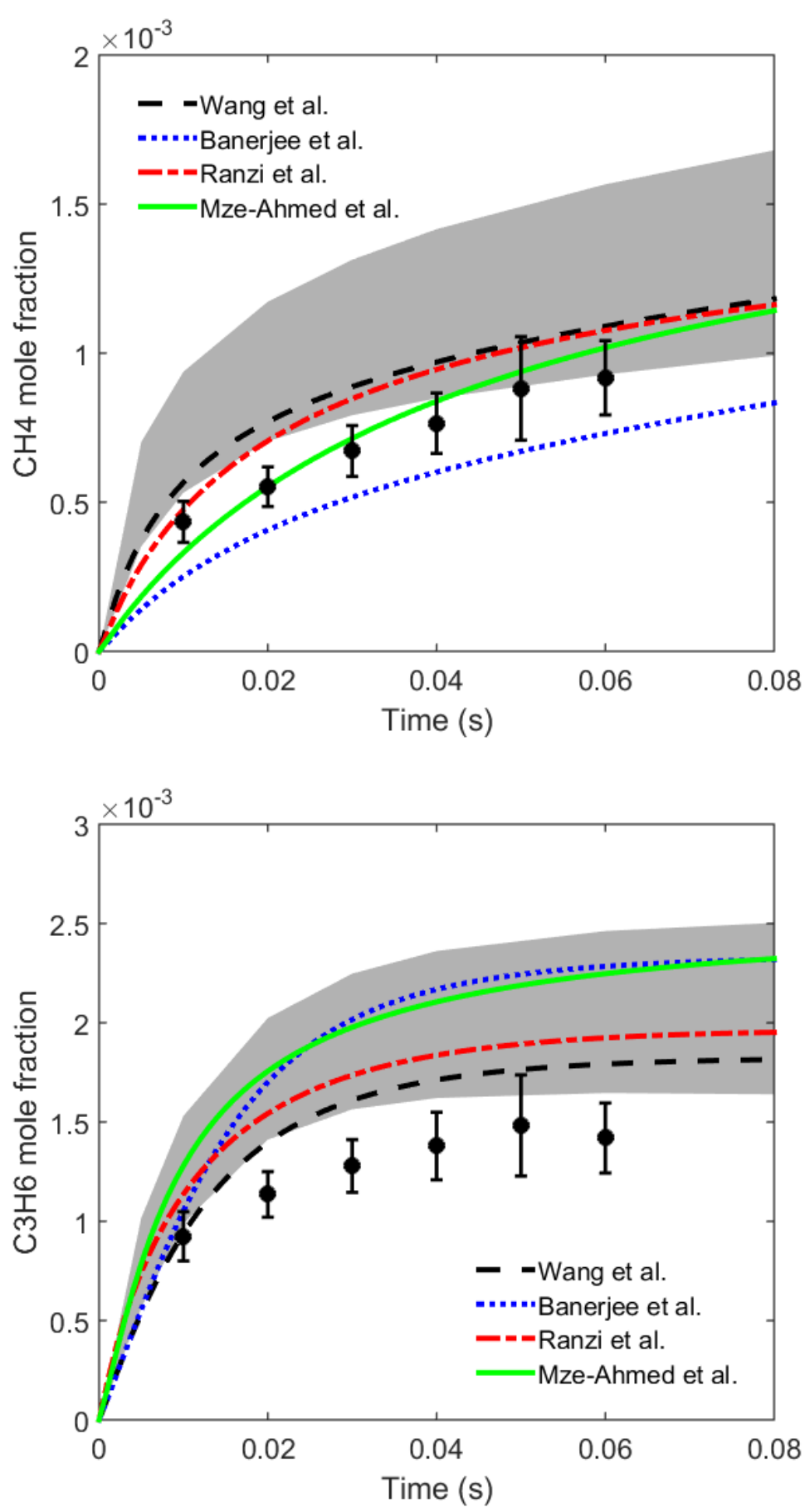

Figure 3.11: Species mole fraction profile as a function of time for n-dodecane pyrolysis in a $1100 \mathrm{~K}$ constant temperature and atmospheric pressure tube reactor. Symbols are UVa reactor experimental data with the error bars indicate uncertainty of the data and lines are model predictions with nominal rates. The shaded area indicates the $2 \sigma$ model prediction uncertainty. 

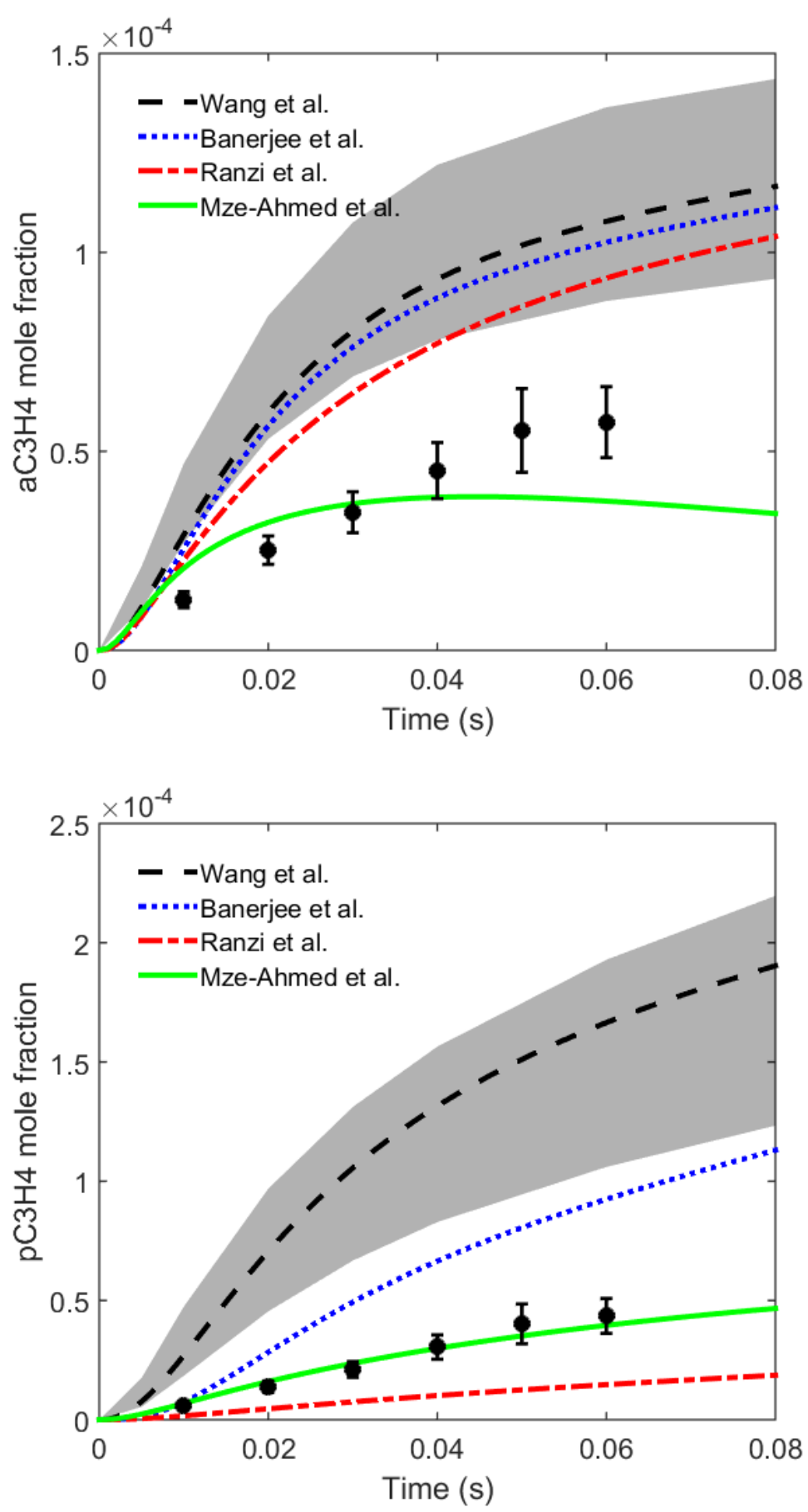

Figure 3.12: Species mole fraction profile as a function of time for n-dodecane pyrolysis in a $1100 \mathrm{~K}$ constant temperature and atmospheric pressure tube reactor. Symbols are UVa reactor experimental data with the error bars indicate uncertainty of the data and lines are model predictions with nominal rates. The shaded area indicates the $2 \sigma$ model prediction uncertainty. 

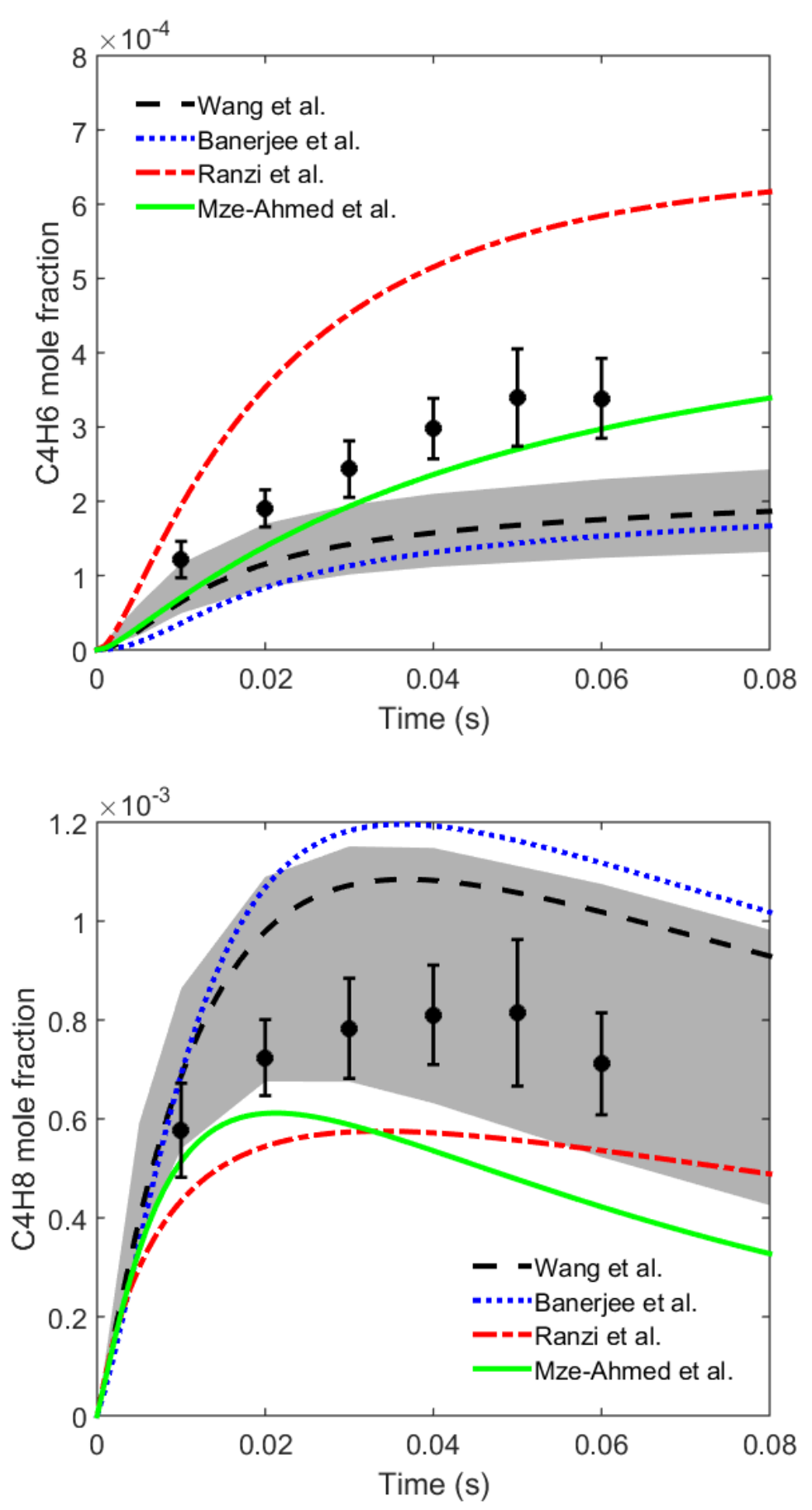

Figure 3.13: Species mole fraction profile as a function of time for n-dodecane pyrolysis in a $1100 \mathrm{~K}$ constant temperature and atmospheric pressure tube reactor. Symbols are UVa reactor experimental data with the error bars indicate uncertainty of the data and lines are model predictions with nominal rates. The shaded area indicates the $2 \sigma$ model prediction uncertainty.

As seen in these figures, the uncertainty of the model prediction is larger than 
the experimental uncertainty in most cases. Also the reaction model developed by Mze-Ahmed et al. [76] has the most agreement between the model and the data.

A significant difference in predicting acetylene mole fraction is observed between the four kinetic models. Acetylene is an important species in chemistry modeling because of being one of the most important precursor molecules for soot formation. To explore the reason of the large difference between the four models, a sensitivity analysis of acetylene formation is performed for all the four considered models. Figures 3.14-3.17 show the sensitivity coefficients of the most important reactions of acetylene concentration among all the investigated models.

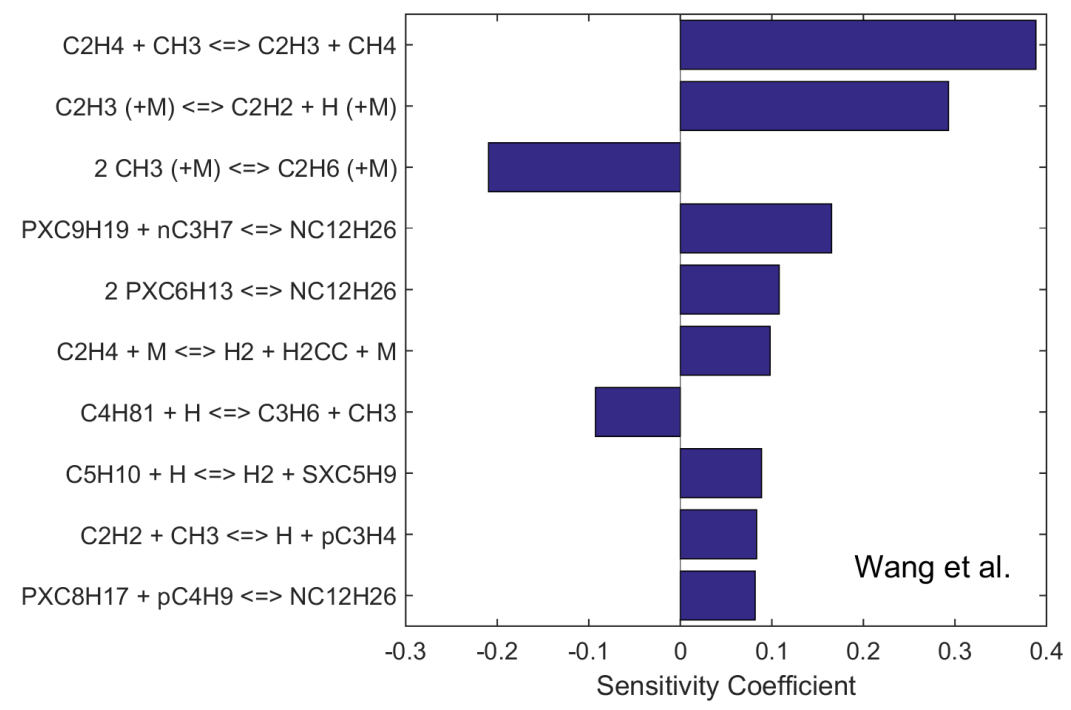

Figure 3.14: Sensitivity of acetylene formation to reaction rates for n-dodecane pyrolysis at $1 \mathrm{~atm}, 1100 \mathrm{~K}$, and $20 \mathrm{~ms}$ using Wang et al. model. The brute-force sensitivity coefficient is calculated by $\left\{d\left[C_{2} H_{2}\right] / d k_{i}\right\} \times\left\{k_{i} /\left[C_{2} H_{2}\right]\right\}$, where $k_{i}$ is the $i$ th reaction rate constant. 


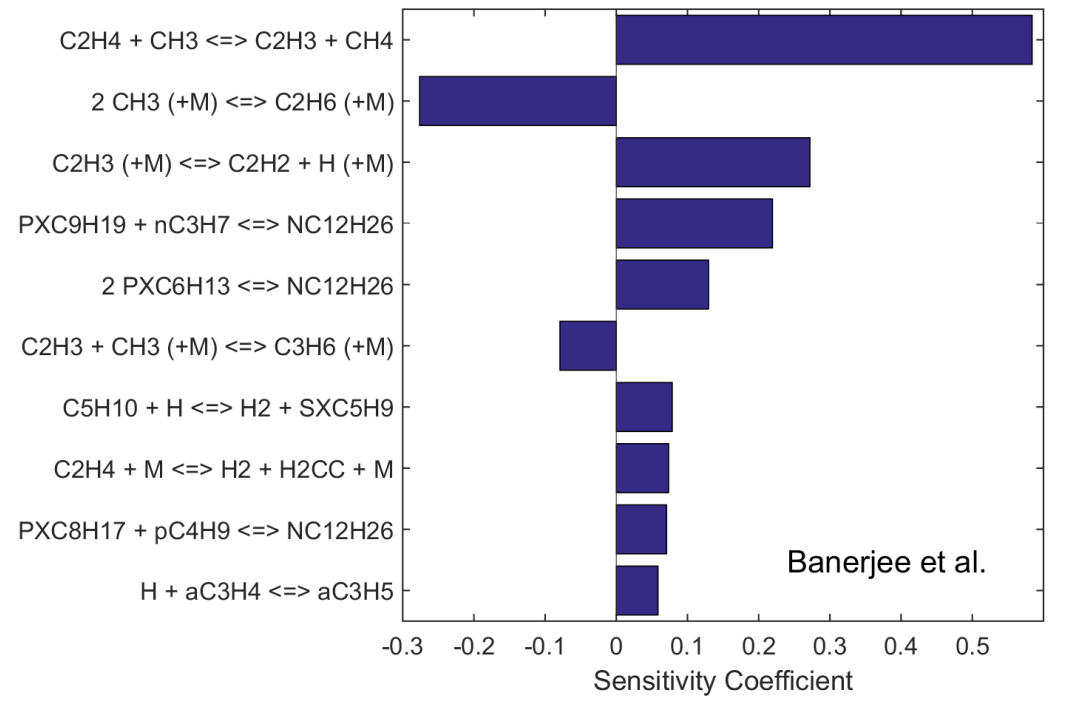

Figure 3.15: Sensitivity of acetylene formation to reaction rates for n-dodecane pyrolysis at $1 \mathrm{~atm}, 1100 \mathrm{~K}$, and $20 \mathrm{~ms}$ using Banerjee et al. model. The brute-force sensitivity coefficient is calculated by $\left\{d\left[C_{2} H_{2}\right] / d k_{i}\right\} \times\left\{k_{i} /\left[C_{2} H_{2}\right]\right\}$, where $k_{i}$ is the $i$ th reaction rate constant.

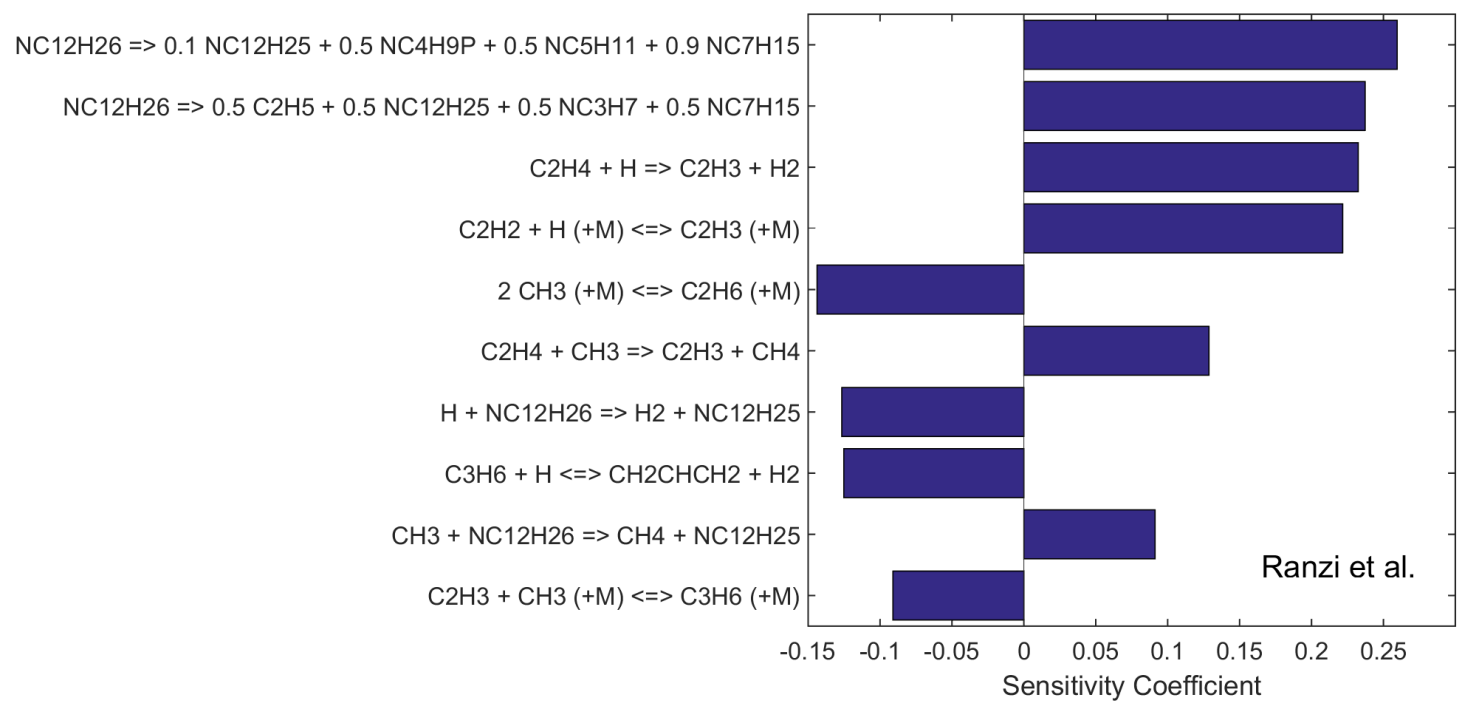

Figure 3.16: Sensitivity of acetylene formation to reaction rates for n-dodecane pyrolysis at $1 \mathrm{~atm}, 1100 \mathrm{~K}$, and $20 \mathrm{~ms}$ using Ranzi et al. model. The brute-force sensitivity coefficient is calculated by $\left\{d\left[C_{2} H_{2}\right] / d k_{i}\right\} \times\left\{k_{i} /\left[C_{2} H_{2}\right]\right\}$, where $k_{i}$ is the $i$ th reaction rate constant. 


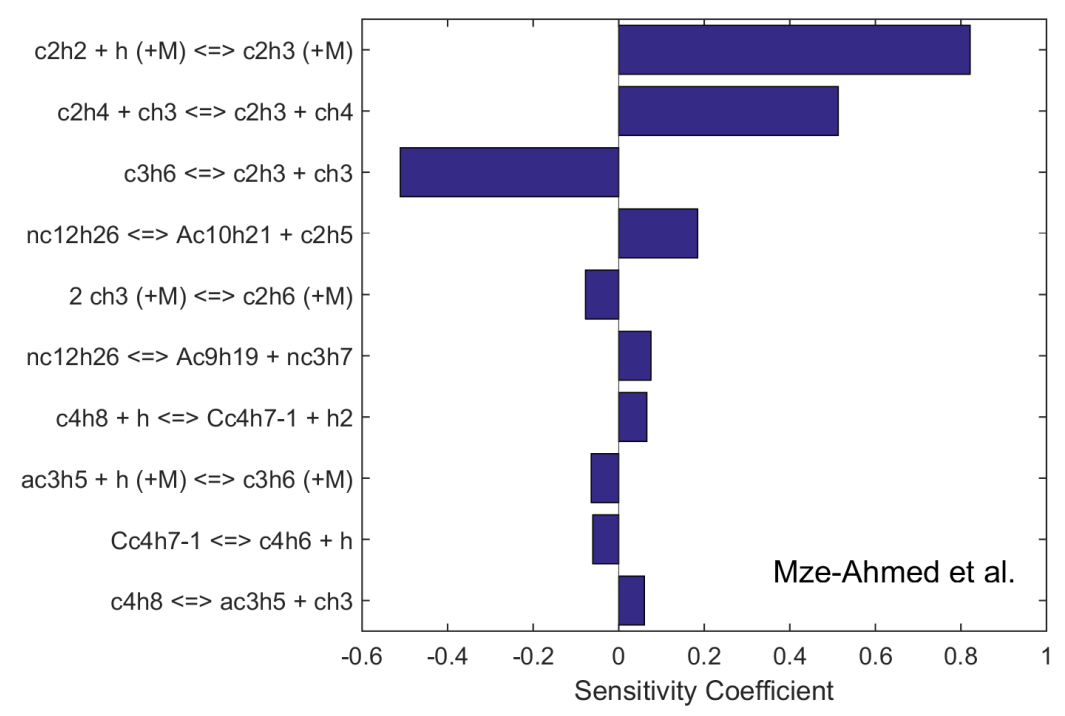

Figure 3.17: Sensitivity of acetylene formation to reaction rates for n-dodecane pyrolysis at $1 \mathrm{~atm}, 1100 \mathrm{~K}$, and $20 \mathrm{~ms}$ using Mze-Ahmed et al. model. The brute-force sensitivity coefficient is calculated by $\left\{d\left[\mathrm{C}_{2} \mathrm{H}_{2}\right] / d k_{i}\right\} \times\left\{k_{i} /\left[\mathrm{C}_{2} \mathrm{H}_{2}\right]\right\}$, where $k_{i}$ is the $i$ th reaction rate constant.

Based on the sensitivity analysis, the reaction

$$
\mathrm{C} 2 \mathrm{H} 4+\mathrm{CH} 3 \leftrightarrow \mathrm{C} 2 \mathrm{H} 3+\mathrm{CH} 4
$$

is one the most important reactions in all the models for acetylene formation at the considered reacting condition. To explore more and find out the reason of this difference between the model predictions, the comparison of the Arrhenius rate of the reaction 3.16 as a functions of temperature in the range $500-2000 \mathrm{~K}$ is calculated and the profile is illustrated in Figure 3.18 for all the four kinetic models. Note that the maximum difference between the rate coefficients are as large as an order of magnitude. This large variation results in almost completely different reaction prediction. Particularly at $1100 \mathrm{~K}$ temperature, the reaction rates from Mze-Ahmed et al., Banerjee et al., Wang et al., and Ranzi et al. are a factor of 2 larger than the other, respectively. The analysis provided here will bring insight to the chemical kinetic model community to focus the model optimization efforts on the most 
influential parameters and targets.

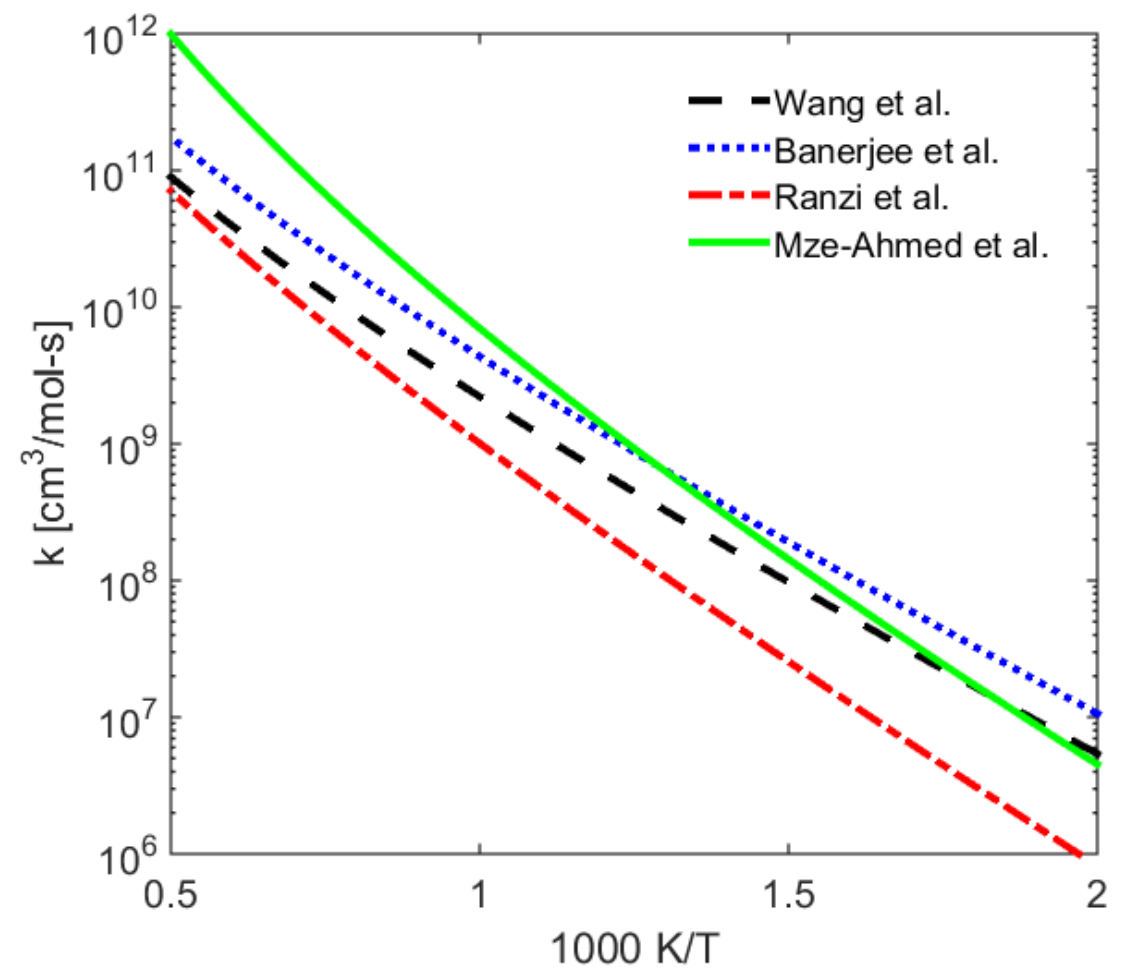

Figure 3.18: Comparison of the recommended reaction rate coefficient of $\mathrm{C} 2 \mathrm{H} 4+\mathrm{CH} 3$ $\rightarrow \mathrm{C} 2 \mathrm{H} 3+\mathrm{CH} 4$ between four kinetic models.

\subsection{Mechanism Reduction for n-Dodecane Pyrol- ysis}

As noted in the previous chapter, the detailed chemical kinetic models of hydrocarbons can have over hundred species and thousands of reactions. Implementing these large models into multi-dimensional reacting flow simulations with complex geometries are computationally prohibitive, even with the current immense computational resources. In a three-dimensional reacting flow simulation, there are five conservation equations for the mass, momentum, and energy. For each species included in the kinetic model, there will be an additional species conservation equation ,similar to 
Eq. 4.3, that needs to be solved. This system of coupled partial differential equations will keep growing as the detailed models are growing. Figure 3.19 which is from Lu and Law [90], shows the size of various detailed mechanisms reported in the literature. The clear observation is that the most recent developed mechanism for real fuels are growing with time. While such large models intend to provide more information about the reaction path and include all the possible elementary reactions, they are computationally impossible to be applied to 3-D reacting flow simulations.

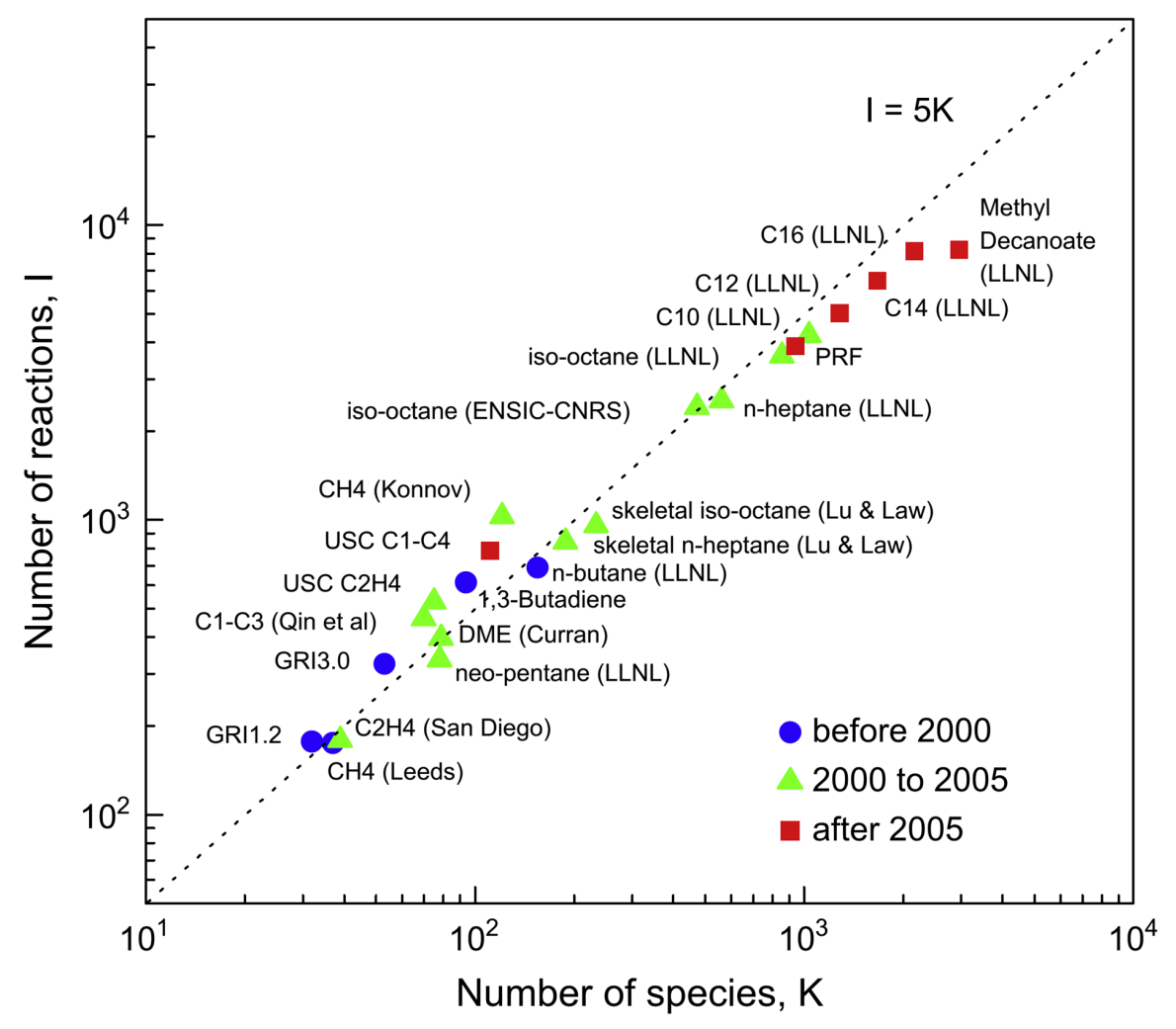

Figure 3.19: Size of different mechanisms for hydrocarbon fuels, with the number of species and reactions included in the mechanisms and the approximate year when the mechanisms were developed (figure from [90]).

Significant reduction of the size of the mechanisms in order to be able to perform multi-dimensional reacting flow simulations are inevitable. The two common methods to reduce mechanisms are principal component analysis (PCA) [91] and directed relation graph (DRG) [92],in which most of the unimportant reactions of the detailed 
model are removed, and a shorter skeletal model is extracted.

In this study, the important reactions are identified using sensitivity analysis and reaction flux analysis, which were described in the prior sections. Skeletal reduced reaction models for n-dodecane pyrolysis are developed based on Principal Component Analysis with Sensitivity (PCAS) [93].

The detailed reaction model Banerjee et al. [19] is used as the starting mechanism. This mechanism is an optimized model for n-dodecane chemical kinetic predictions and consist of 196 species and 1478 reactions. The skeletal model is used for the twodimensional reacting flow simulation of n-dodecane pyrolysis in laminar tube reactor. Therefore, major species from n-dodecane pyrolysis are selected as the target functions in the analysis. The initial selected targets were n-dodecane, ethylene, hydrogen, methane, and ethane mole fractions yield. However, after the reduction process, high errors were observed even with large number of species. With more careful analysis a number of intermediate species were added to the target functions in the analysis. The species considered as the important intermediate species were: C8H17, C10H20, C2H2, C7H15, C8H16, aC3H4. By including these species sensitivities in the analysis, skeletal models with highest accuracy of prediction of species concentration were extracted for n-dodecane pyrolysis.

Figure 3.20 shows the $L^{2}$-norm error of model predictions for skeletal models with included number of species ranging from 35 to 65 . The $L^{2}$-norm error is defined as

$$
\|\epsilon\|_{2}=\sqrt{\sum_{i=1}^{n}\left(\frac{f_{\text {skeletal }}-f_{\text {detail }}}{f_{\text {detail }}}\right)^{2}}
$$

The error indicates the prediction difference between the detailed model and the adopted skeletal model with selected number of species. The error is calculated based on the concentration of five major species of n-dodecane, hydrogen, ethylene, ethane, methane. The results from the developed models suggested less than $2 \%$ error for 
reduced models with more than 50 species included. The error is less than $5 \%$ for skeletal models with more than 42 species included. After this point, any more reduction increases the error to higher thresholds and needs careful attention when implementing.

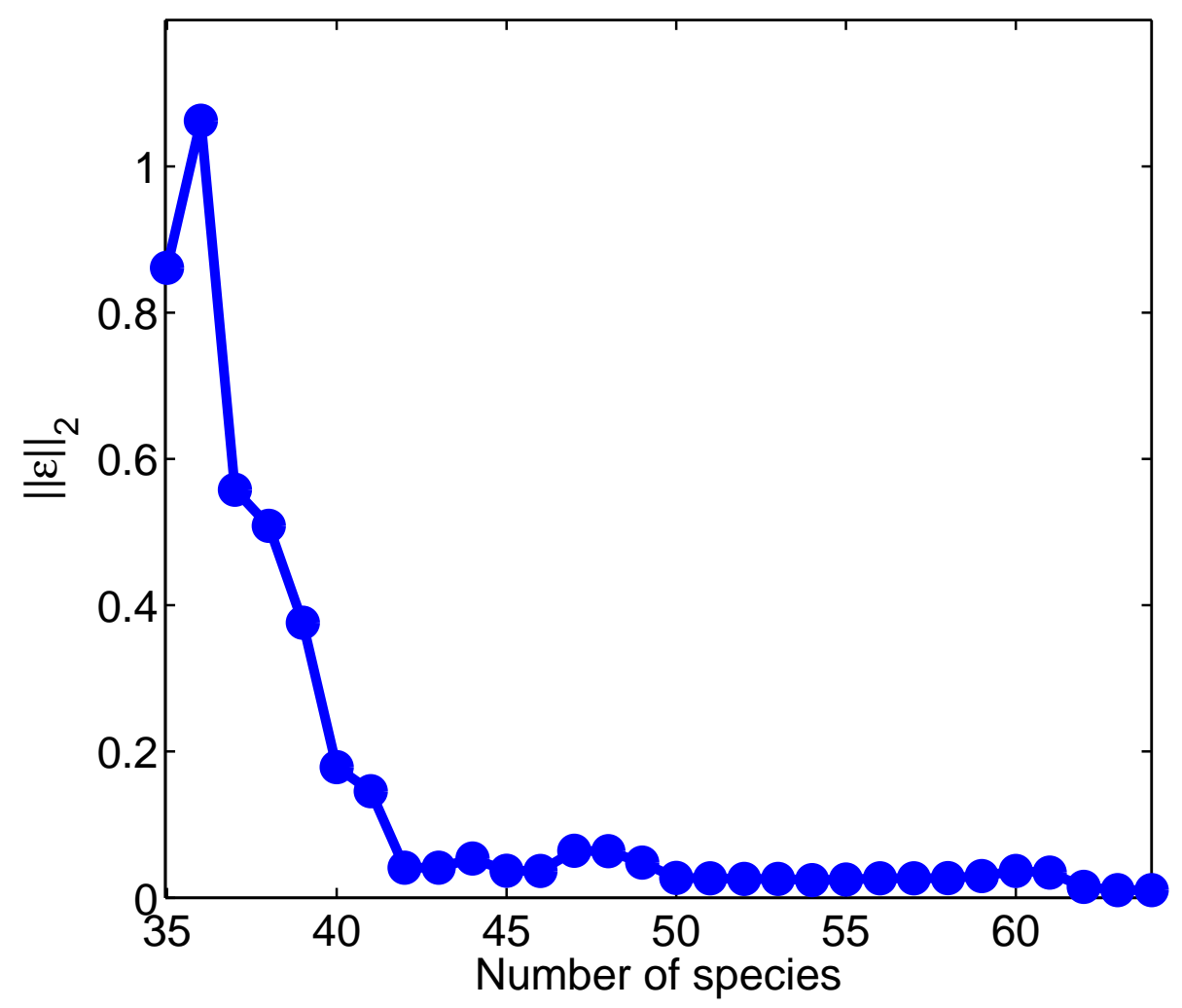

Figure 3.20: The $L^{2}$-norm error for the prediction of n-dodecane pyrolysis vs. the number of species in the reduced order skeletal model.

To illustrate the performance of the developed skeletal models, the predictions of 4 models are shown in Figures 3.21 and 3.22 for n-dodecane pyrolysis at the UVa tube reactor experiment operating condition. The detail model is compared against three skeletal models with number of species 50, 40, and 36, respectively. It is clear that the skeletal model with 50 species is in complete agreement with the detailed model for the prediction of all the major species. Also the 40 species skeletal model has an acceptable prediction for most of the targets except hydrogen. However, the 32 species skeletal model prediction shows a significant departure from the detailed 
model result for all the major species. This departure was quantified by the error calculation for a range of species in Figure 3.20.
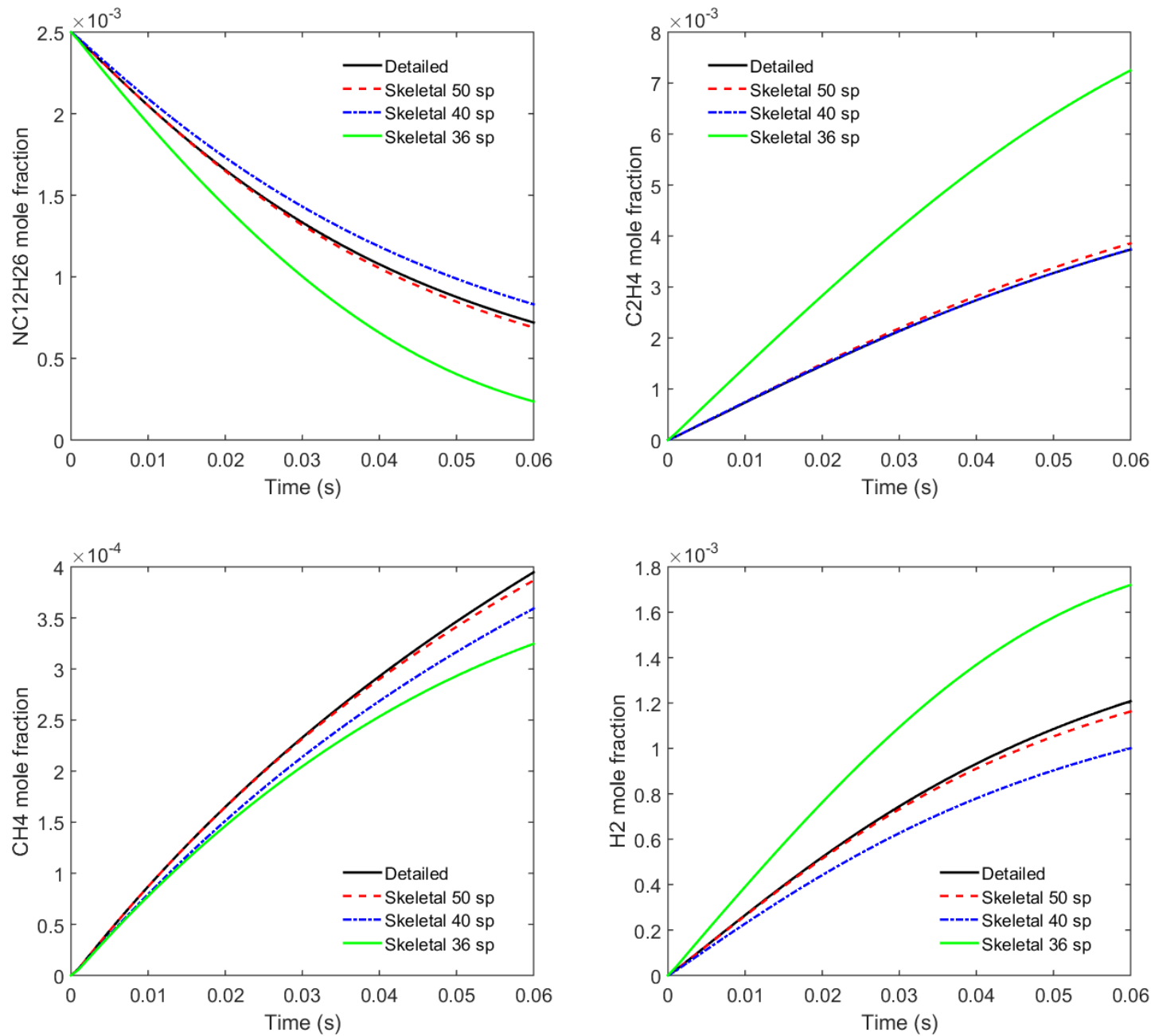

Figure 3.21: Comparison of the predicted species mole fraction profiles between the detailed model and skeletal models with 50, 40, and 36 species. The conditions are for $0.25 \% \mathrm{n}$-dodecane pyrolysis in the atmospheric pressure tube reactor at $1050 \mathrm{~K}$. 

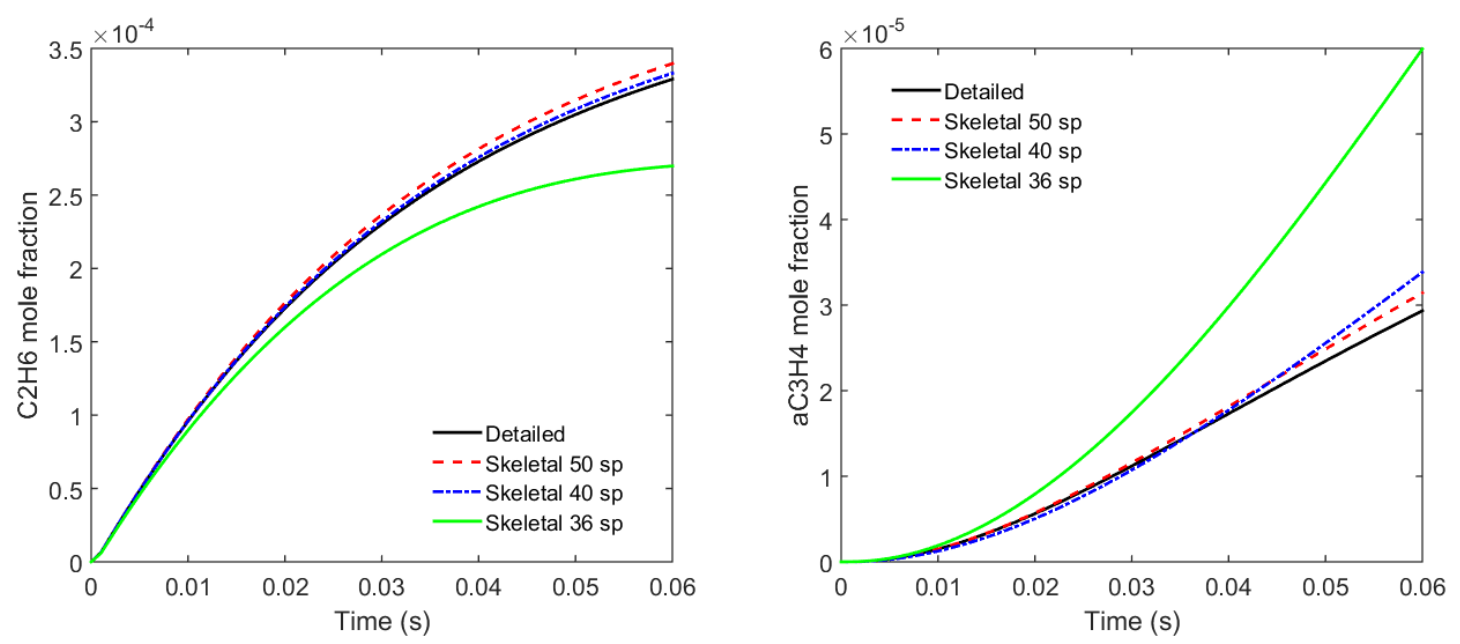

Figure 3.22: Comparison of the predicted species mole fraction profiles between the detailed model and skeletal models with 50, 40, and 36 species. The conditions are for $0.25 \% \mathrm{n}$-dodecane pyrolysis in the atmospheric pressure tube reactor at $1050 \mathrm{~K}$.

Based on this analysis, the optimum reduced model with 50 species was selected to be implemented in the two-dimensional tube reactor flow simulation. The details of this model are given in Appendix B. 


\subsection{Ethane Pyrolysis}

For relatively well known ethane pyrolysis kinetic models [69], brute force sensitivity analysis has been performed at atmospheric pressure and $1100 \mathrm{~K}$. For a case where the reactor flow velocity corresponded to an overall residence time of $100 \mathrm{~ms}$, the most sensitive reactions and their sensitivity coefficients are shown in Figure 3.23 at a residence time of $50 \mathrm{~ms}$. The target function to calculate the sensitivity coefficients is the ethane mole fraction.

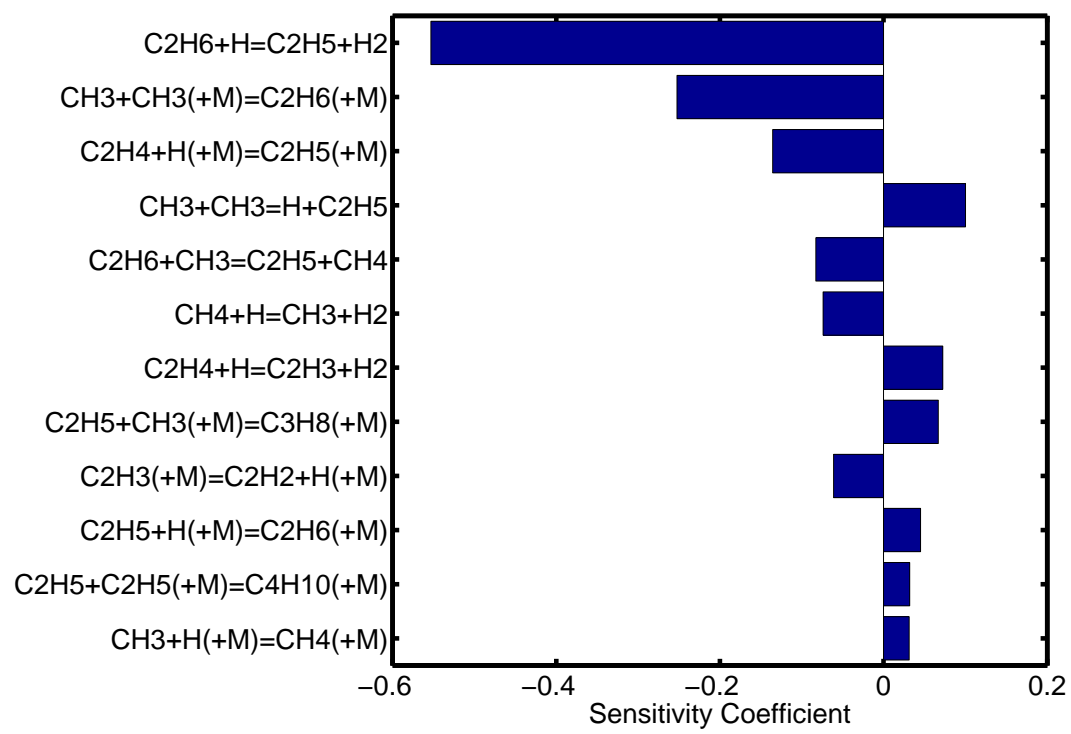

Figure 3.23: Normalized local sensitivity coefficients of $\mathrm{C}_{2} \mathrm{H}_{6}$ pyrolysis at $\mathrm{p}=1$ atm and $\mathrm{T}=1100 \mathrm{~K}$ evaluated at $50 \mathrm{~ms}$. The sensitivity is defined as $\left\{d\left[C_{2} H_{6}\right] / d k_{i}\right\} \times$ $\left\{k_{i} /\left[C_{2} H_{6}\right]\right\}$, where $k_{i}$ is the $i$ th reaction rate constant.

Besides the local sensitivities, reaction path flux analysis was performed to understand fuel pyrolysis in the mixing region and along the tube reactor. Fig. 3.24 shows the initiation reaction for ethane pyrolysis at $0.2 \mathrm{~ns}$. In the case of ethane pyrolysis, at $1100 \mathrm{~K}$ and at small time scales of the order of $0.1 \mathrm{~ms}$, Fig. 3.25 shows that the hydrogen abstraction from ethane is primarily controlled by reactions with $\mathrm{H}$-atoms (74\%) and $\mathrm{CH}_{3}(8 \%)$. The dominant initiation reaction (at ns time scales), $\mathrm{C}_{2} \mathrm{H}_{6}(+\mathrm{M}) \rightarrow 2 \mathrm{CH}_{3}(+\mathrm{M})$, contributes only $18 \%$ of the overall ethane decomposition 
at $0.1 \mathrm{~ms}$. Out of the $82 \%$ of ethane converted to ethyl radical, only $8 \%$ lead to formation of methane, while $100 \%$ of ethyl radical leads to formation of ethylene. While the overall ethane consumption is several orders of magnitude smaller than the peak rate that occur at about $50 \mathrm{~ms}$, the trend of high $\mathrm{C}_{2} \mathrm{H}_{4}$ to $\mathrm{CH}_{4}$ formation at early times is consistent with shock tube data by Tranter et al. [71].

\section{$\mathrm{C} 2 \mathrm{H} 6 \stackrel{+\mathrm{M} 100 \%}{\longrightarrow} \mathrm{CH} 3$}

Figure 3.24: Reaction path flux analysis for $\mathrm{C}_{2} \mathrm{H}_{6}$ pyrolysis at $\mathrm{p}=1 \mathrm{~atm}, \mathrm{~T}=1100 \mathrm{~K}$ and $0.2 \mathrm{~ns}$.

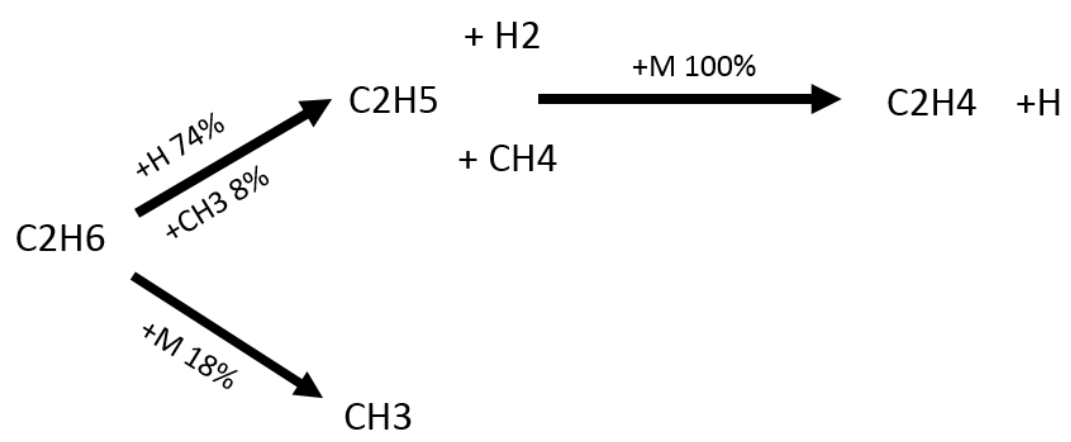

Figure 3.25: Reaction path flux analysis for $\mathrm{C}_{2} \mathrm{H}_{6}$ pyrolysis at $\mathrm{p}=1 \mathrm{~atm}, \mathrm{~T}=1100 \mathrm{~K}$ and $0.1 \mathrm{~ms}$.

At longer residence times, i.e. of the order $50 \mathrm{~ms}$, ethane pyrolysis peaks with overall rate of about $10^{-6} \mathrm{mols} / \mathrm{cm}^{3} / \mathrm{s}$ (note: in reaction flux plots, the thickness of the arrows correspond to molar production rates), the main consumption is through ethyl formation with $\mathrm{H}$-atoms, followed by ethylene formation. A small flux of ethylene is converted to vinyl radical by hydrogen abstraction. Once vinyl radicals are formed, it can react with ethylene leading to the formation of 1,3-butadiene (30\%) and with hydrogen abstraction to form acetylene (70\%). The local sensitivity results shown in Fig. 3.23 at $50 \mathrm{~ms}$ has identified these key reactions with most sensitive reaction being $\mathrm{C}_{2} \mathrm{H}_{6}+\mathrm{H} \rightarrow \mathrm{C}_{2} \mathrm{H}_{5}+\mathrm{H}_{2}$. This particular reaction is known to have an uncertainty 
factor of 2, hence any accurate experimental data may offer to reduce the uncertainty of this particular reaction further.

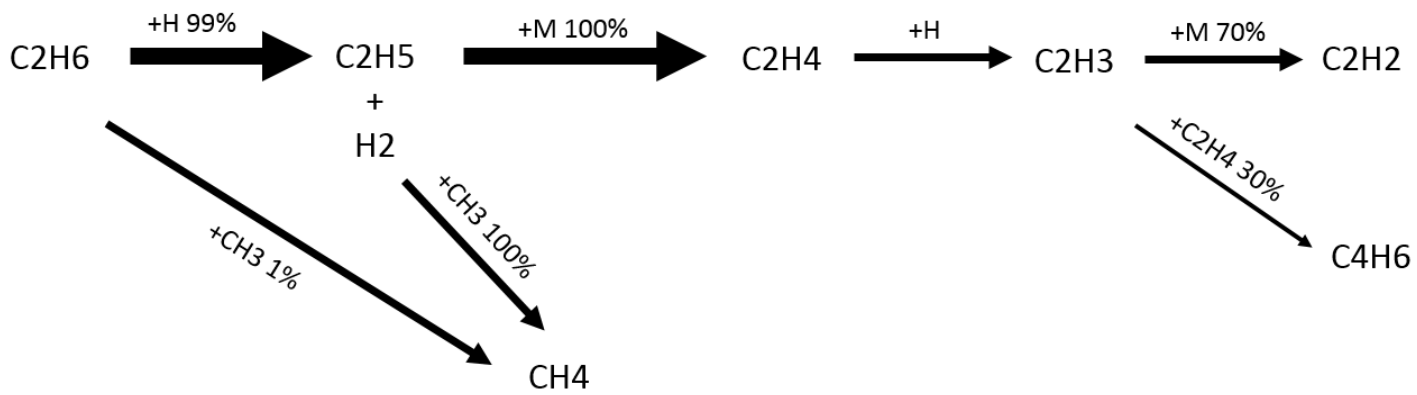

Figure 3.26: Reaction path flux analysis for $\mathrm{C}_{2} \mathrm{H}_{6}$ pyrolysis at $\mathrm{p}=1$ atm, $\mathrm{T}=1100 \mathrm{~K}$ and $50 \mathrm{~ms}$.

At longest residence times explored in MFTR, namely at $100 \mathrm{~ms}$, ethane pyrolysis has already peaked as well as the ethylene formation, as shown by the line thickness in Fig. 3.27. On the other hand methane formation continues to progress at a similar rate, which leads to a lower $\mathrm{C}_{2} \mathrm{H}_{4} / \mathrm{CH}_{4}$. Furthermore, 1,3-butadiene formed leads to formation of allene and propyne, which were also observed in the MFTR at longer residence times. For higher temperatures of the order of $1200 \mathrm{~K}$ and residence times of the order of $500 \mathrm{~ms}$, experiments suggest formation of soot or coke. Theoretical investigations of reaction pathway analysis and aerosol particle dynamics are beyond the scope of this work.

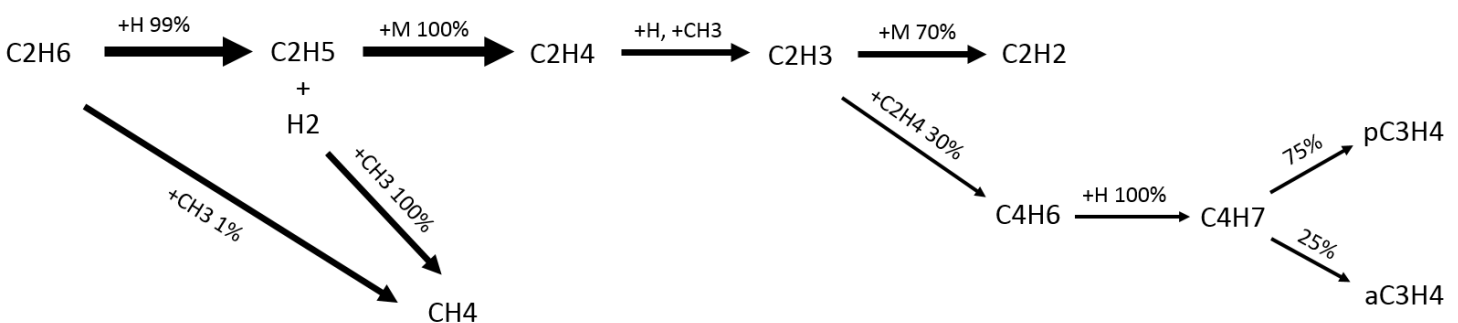

Figure 3.27: Reaction path flux analysis for $\mathrm{C}_{2} \mathrm{H}_{6}$ pyrolysis at $\mathrm{p}=1$ atm, $\mathrm{T}=1100 \mathrm{~K}$ and $100 \mathrm{~ms}$.

Figures 3.28-3.36 show the Monte Carlo simulation of the ethane pyrolysis at 
atmospheric pressure and $1100 \mathrm{~K}$ condition.

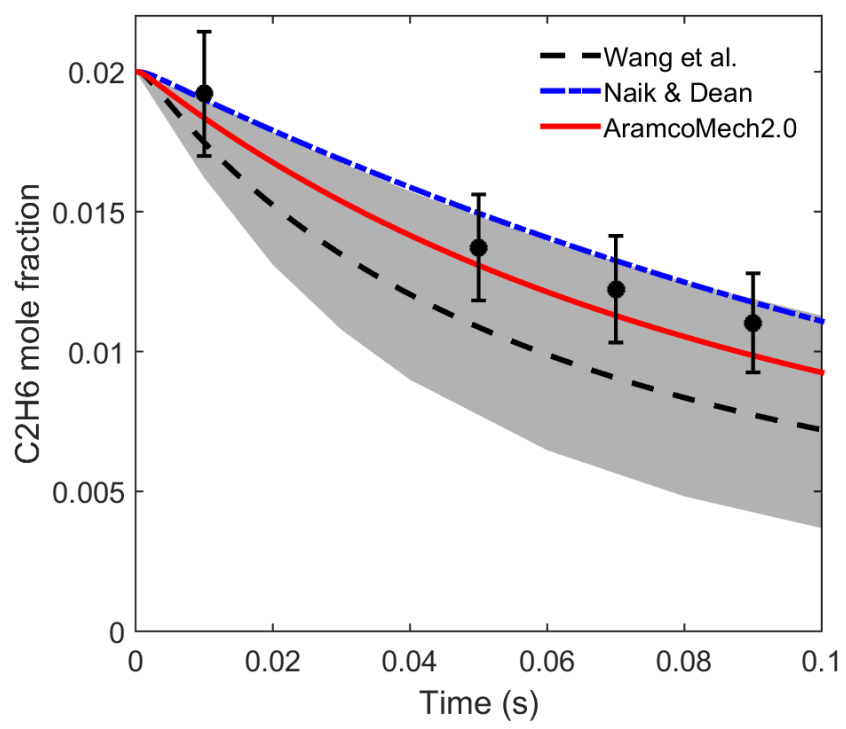

Figure 3.28: Mole fraction as a function of temperature

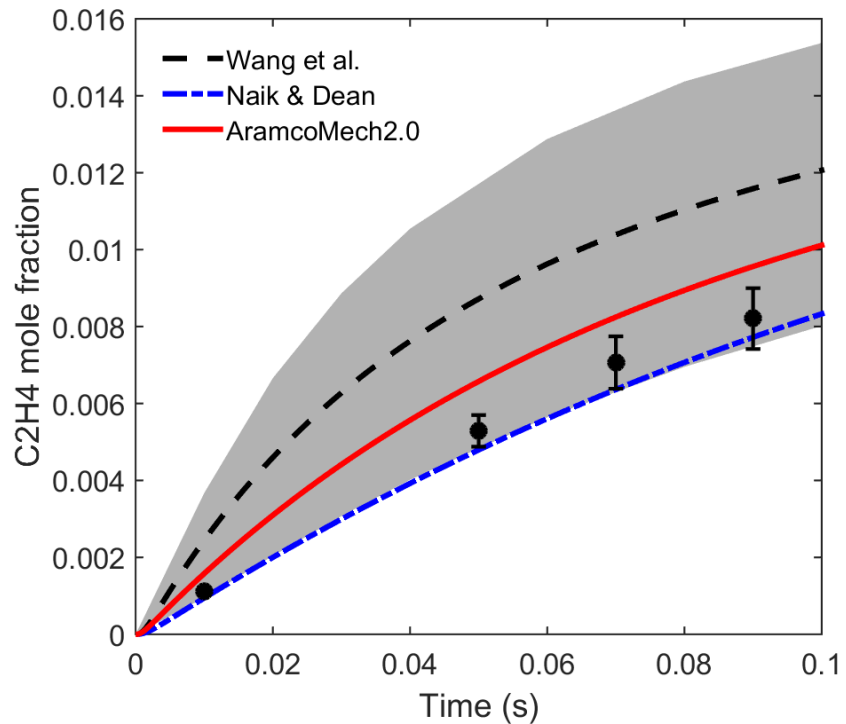

Figure 3.29: Mole fraction as a function of temperature 


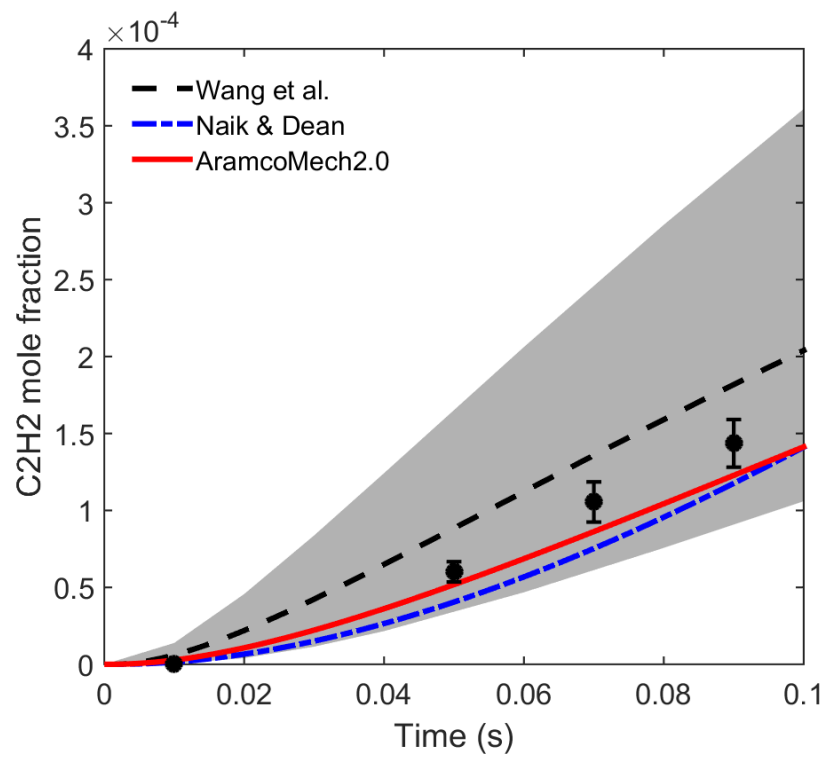

Figure 3.30: Mole fraction as a function of temperature

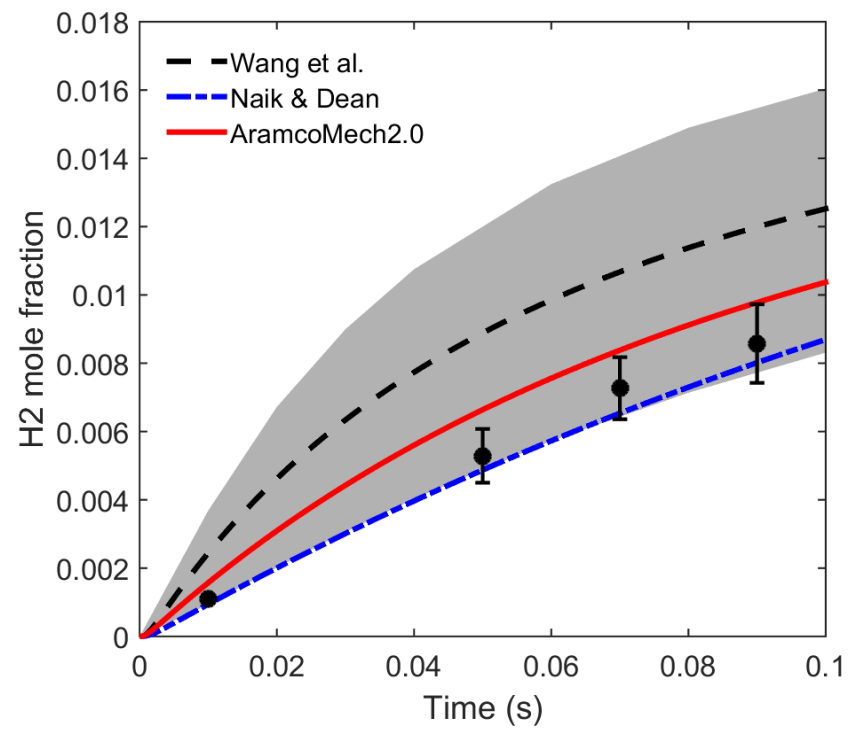

Figure 3.31: Mole fraction as a function of temperature 


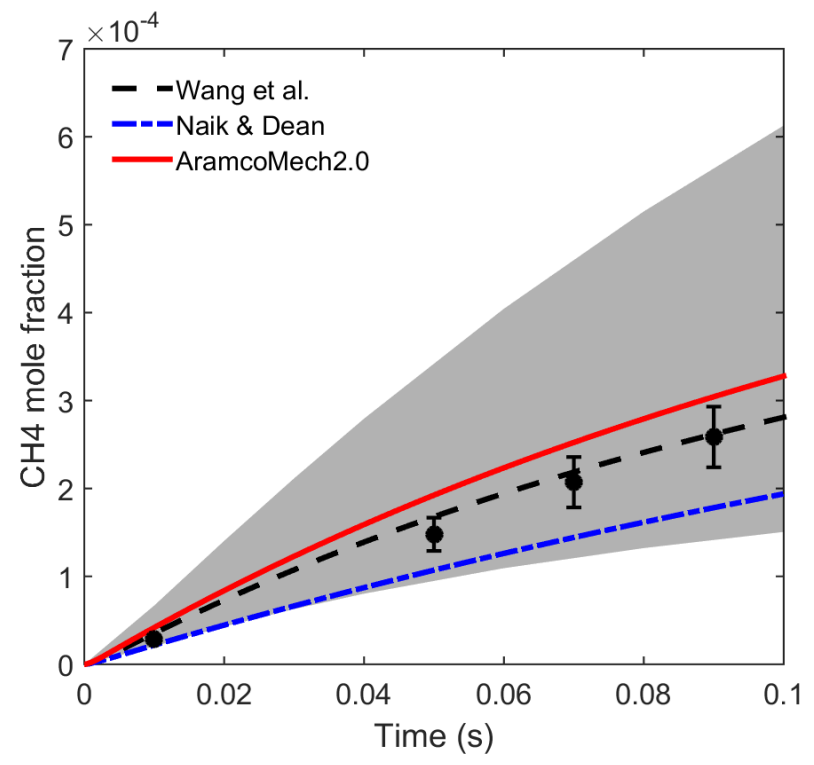

Figure 3.32: Mole fraction as a function of temperature

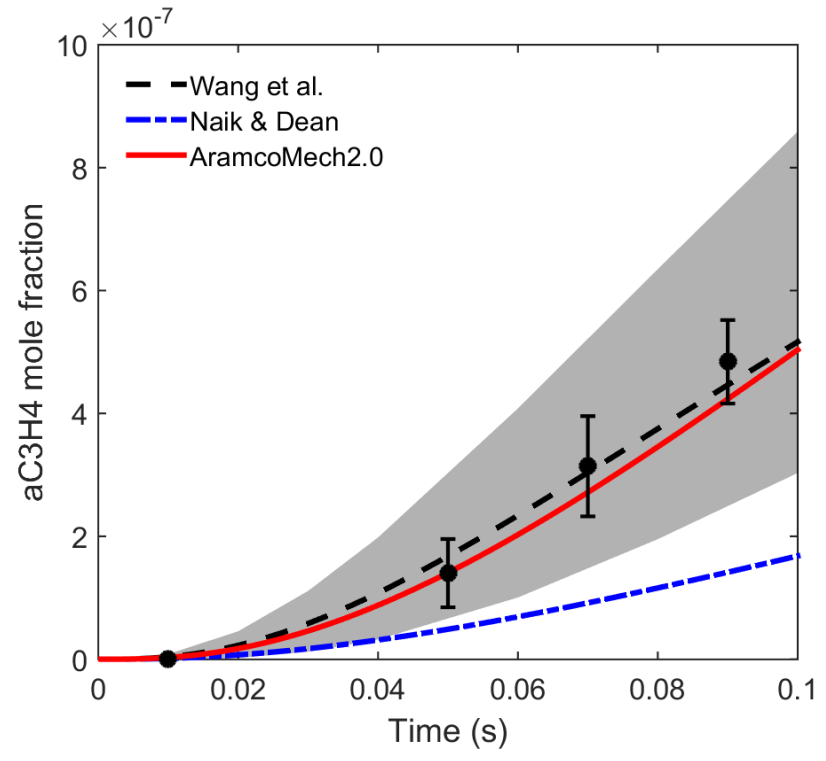

Figure 3.33: Mole fraction as a function of temperature 


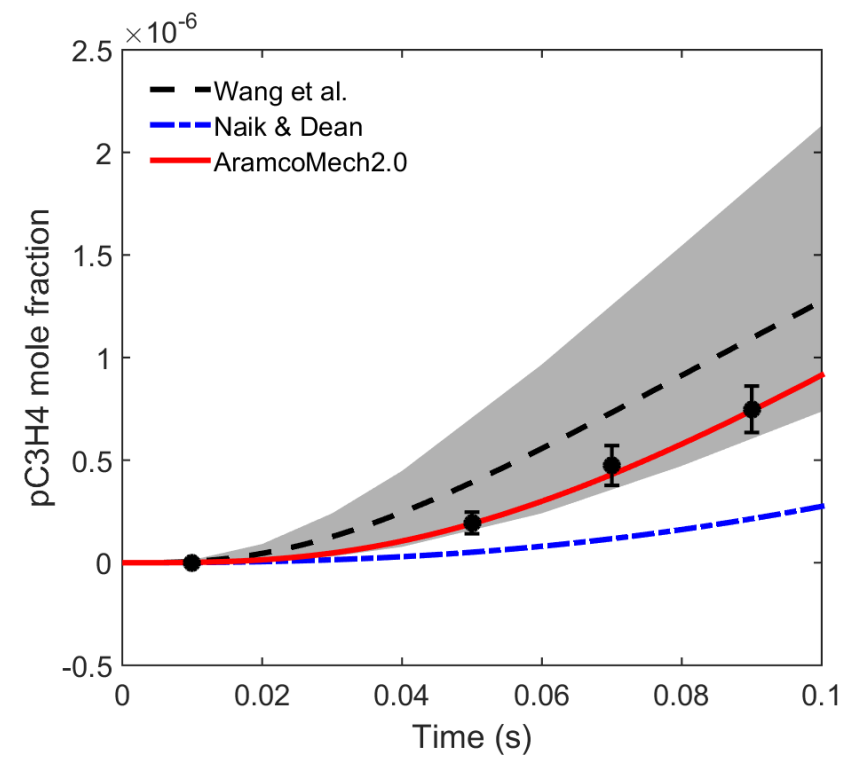

Figure 3.34: Mole fraction as a function of temperature

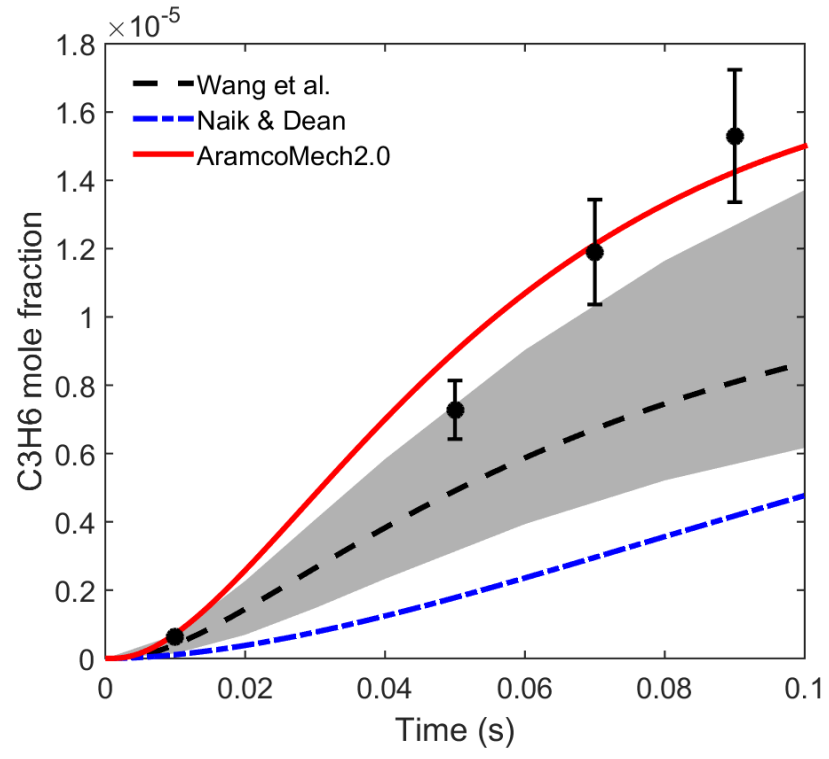

Figure 3.35: Mole fraction as a function of temperature 


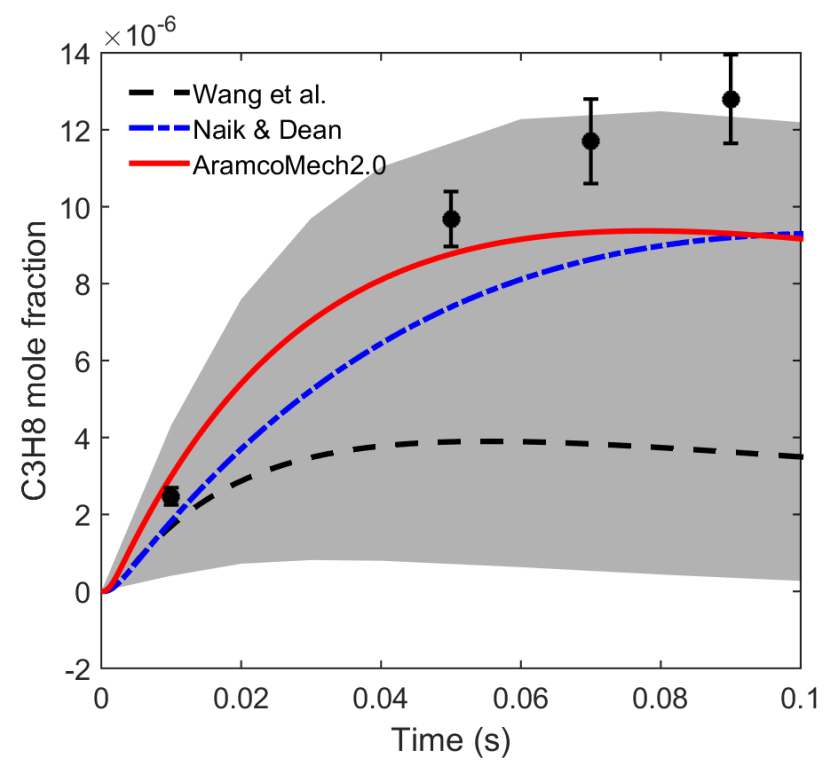

Figure 3.36: Mole fraction as a function of temperature

The Monte Carlo simulation to quantify the uncertainty of the chemical kinetic parameters are performed by selecting the most sensitive reactions in the mechanism to be perturbed. Tables 3.2 and 3.3 list these selected sensitive reactions with their uncertainty factors for ethane and n-butane pyrolysis, respectively.

Table 3.2: Reactions selected for uncertainty propagation investigation for ethane pyrolysis.

\begin{tabular}{lc}
\hline Reaction & Uncertainty Factor \\
\hline $2 \mathrm{CH} 3(+\mathrm{M})=\mathrm{C} 2 \mathrm{H} 6(+\mathrm{M})$ & 2 \\
$\mathrm{C} 2 \mathrm{H} 4+\mathrm{H}(+\mathrm{M})=\mathrm{C} 2 \mathrm{H} 5(+\mathrm{M})$ & 3 \\
$\mathrm{C} 2 \mathrm{H} 6+\mathrm{H}=\mathrm{C} 2 \mathrm{H} 5+\mathrm{H} 2$ & 1.5 \\
$\mathrm{C} 2 \mathrm{H} 6+\mathrm{CH} 3=\mathrm{C} 2 \mathrm{H} 5+\mathrm{CH} 4$ & 5 \\
$2 \mathrm{CH} 3=\mathrm{H}+\mathrm{C} 2 \mathrm{H} 5$ & 2 \\
$2 \mathrm{C} 2 \mathrm{H} 5(+\mathrm{M})=\mathrm{C} 4 \mathrm{H} 10(+\mathrm{M})$ & 3 \\
$\mathrm{C} 2 \mathrm{H} 5+\mathrm{CH} 3(+\mathrm{M})=\mathrm{C} 3 \mathrm{H} 8(+\mathrm{M})$ & 2 \\
$\mathrm{C} 2 \mathrm{H} 5+\mathrm{H}(+\mathrm{M})=\mathrm{C} 2 \mathrm{H} 6(+\mathrm{M})$ & 2 \\
$\mathrm{CH} 3+\mathrm{H}(+\mathrm{M})=\mathrm{CH} 4(+\mathrm{M})$ & 3 \\
$\mathrm{C} 2 \mathrm{H} 5+\mathrm{H}=\mathrm{C} 2 \mathrm{H} 4+\mathrm{H} 2$ & 2 \\
$\mathrm{CH} 4+\mathrm{H}=\mathrm{CH} 3+\mathrm{H} 2$ & 2 \\
$\mathrm{C} 2 \mathrm{H} 4+\mathrm{H}=\mathrm{C} 2 \mathrm{H} 3+\mathrm{H} 2$ & 1.5 \\
$\mathrm{C} 2 \mathrm{H} 3(+\mathrm{M})=\mathrm{C} 2 \mathrm{H} 2+\mathrm{H}(+\mathrm{M})$ & 5 \\
$\mathrm{C} 2 \mathrm{H} 4+\mathrm{CH} 3=\mathrm{C} 2 \mathrm{H} 3+\mathrm{CH} 4$ & 3 \\
$\mathrm{C} 2 \mathrm{H} 3+\mathrm{H}=\mathrm{C} 2 \mathrm{H} 2+\mathrm{H} 2$ & \\
\hline
\end{tabular}


Table 3.3: Reactions selected for uncertainty propagation investigation for n-butane pyrolysis.

\begin{tabular}{lc}
\hline Reaction & Uncertainty Factor \\
\hline 2C2H5 $(+\mathrm{M})=\mathrm{C} 4 \mathrm{H} 10(+\mathrm{M})$ & 2 \\
$\mathrm{C} 4 \mathrm{H} 10+\mathrm{CH} 3=\mathrm{sC} 4 \mathrm{H} 9+\mathrm{CH} 4$ & 3 \\
$\mathrm{C} 4 \mathrm{H} 10+\mathrm{CH} 3=\mathrm{pC} 4 \mathrm{H} 9+\mathrm{CH} 4$ & 3 \\
$\mathrm{C} 3 \mathrm{H} 6+\mathrm{CH} 3(+\mathrm{M})=\mathrm{sC} 4 \mathrm{H} 9(+\mathrm{M})$ & 2 \\
$\mathrm{C} 4 \mathrm{H} 10+\mathrm{H}=\mathrm{pC} 4 \mathrm{H} 9+\mathrm{H} 2$ & 3 \\
$\mathrm{nC} 3 \mathrm{H} 7+\mathrm{CH} 3(+\mathrm{M})=\mathrm{C} 4 \mathrm{H} 10(+\mathrm{M})$ & 2 \\
2CH3 $(+\mathrm{M})=\mathrm{C} 2 \mathrm{H} 6(+\mathrm{M})$ & 2 \\
$\mathrm{C} 4 \mathrm{H} 10+\mathrm{H}=\mathrm{sC} 4 \mathrm{H} 9+\mathrm{H} 2$ & 3 \\
$\mathrm{C} 4 \mathrm{H} 82+\mathrm{H}(+\mathrm{M})=\mathrm{sC} 4 \mathrm{H} 9(+\mathrm{M})$ & 3 \\
$\mathrm{C} 4 \mathrm{H} 81+\mathrm{H}(+\mathrm{M})=\mathrm{sC} 4 \mathrm{H} 9(+\mathrm{M})$ & 3 \\
$\mathrm{CH} 4+\mathrm{H}=\mathrm{CH} 3+\mathrm{H} 2$ & 2 \\
$\mathrm{C} 3 \mathrm{H} 6+\mathrm{H}=\mathrm{aC} 3 \mathrm{H} 5+\mathrm{H} 2$ & 2 \\
$\mathrm{C} 2 \mathrm{H} 5+\mathrm{CH} 3(+\mathrm{M})=\mathrm{C} 3 \mathrm{H} 8(+\mathrm{M})$ & 3 \\
$\mathrm{C} 4 \mathrm{H} 81+\mathrm{H}=\mathrm{C} 3 \mathrm{H} 6+\mathrm{CH} 3$ & 5 \\
$\mathrm{C} 3 \mathrm{H} 6+\mathrm{H}=\mathrm{C} 2 \mathrm{H} 4+\mathrm{CH} 3$ & 2 \\
\hline
\end{tabular}

\section{6 n-Butane Pyrolysis}

Brute force sensitivity analysis has been performed for n-butane pyrolysis at atmospheric pressure and $1100 \mathrm{~K}$ using JetSurf2.0 [69] kinetic model. For a case where the reactor flow velocity corresponded to an overall residence time of $100 \mathrm{~ms}$, the most sensitive reactions and their sensitivity coefficients are shown in Figure 3.37 at a residence time of $50 \mathrm{~ms}$. The target function to calculate the sensitivity coefficients is the n-butane mole fraction. 


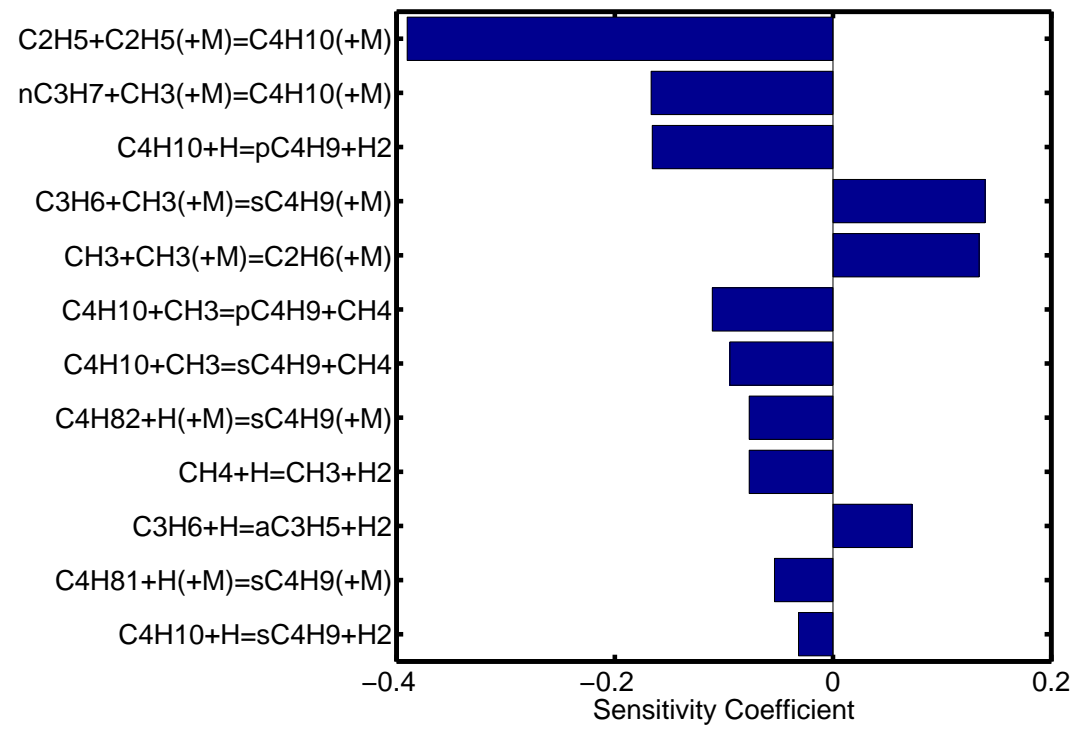

Figure 3.37: Normalized local sensitivity coefficients of $\mathrm{C}_{4} \mathrm{H}_{10}$ pyrolysis at $\mathrm{p}=1 \mathrm{~atm}$ and $\mathrm{T}=1100 \mathrm{~K}$ evaluated at $50 \mathrm{~ms}$. The sensitivity is defined as $\left\{d\left[C_{4} H_{10}\right] / d k_{i}\right\} \times$ $\left\{k_{i} /\left[C_{4} H_{10}\right]\right\}$, where $k_{i}$ is the $i$ th reaction rate constant.

Figures 3.38-3.40 show the reaction pathway analysis for n-butane pyrolysis at $1100 \mathrm{~K}$ for three different residence times. Unlike ethane, at ns time scales the two dominant initiation reactions are $\mathrm{C}_{4} \mathrm{H}_{10}(+\mathrm{M}) \rightarrow 2 \mathrm{C}_{2} \mathrm{H}_{5}(+\mathrm{M})(57 \%)$ and $\mathrm{C}_{4} \mathrm{H}_{10}$ $(+\mathrm{M}) \rightarrow \mathrm{n}^{-} \mathrm{C}_{3} \mathrm{H}_{7}+\mathrm{CH}_{3}(+\mathrm{M})(43 \%)$. At time $0.1 \mathrm{~ms}$ (see Fig. 3.39 ), $57 \%$ of the butane consumption results in the formation of s- $\mathrm{C}_{4} \mathrm{H}_{9}$ through $\mathrm{C}_{4} \mathrm{H}_{10}+\mathrm{H} \rightarrow$ $\mathrm{sC}_{4} \mathrm{H}_{9}+\mathrm{H}_{2}$ and $\mathrm{C}_{4} \mathrm{H}_{10}+\mathrm{CH}_{3} \rightarrow \mathrm{sC}_{4} \mathrm{H}_{9}+\mathrm{CH}_{4}$. About $20 \%$ forms $\mathrm{p}-\mathrm{C}_{4} \mathrm{H}_{9}$ through $\mathrm{C}_{4} \mathrm{H}_{10}+\mathrm{H} \rightarrow \mathrm{pC}_{4} \mathrm{H}_{9}+\mathrm{H}_{2}$ and $\mathrm{C}_{4} \mathrm{H}_{10}+\mathrm{CH}_{3} \rightarrow \mathrm{pC}_{4} \mathrm{H}_{9}+\mathrm{CH}_{4}$. The other $22 \%$ is through the same butane decomposition initiation reactions. The main consumption pathways for $\mathrm{s}^{-} \mathrm{C}_{4} \mathrm{H}_{9}$ include decomposition to propene and methyl radical (63\%), and hydrogen abstraction to form 1-butene and 2-butene (about 20\% each). All the other intermediate species $\left(\mathrm{p}-\mathrm{C}_{4} \mathrm{H}_{9}, \mathrm{C}_{2} \mathrm{H}_{5}, \mathrm{n}-\mathrm{C}_{3} \mathrm{H}_{7}\right)$ contribute in the formation of ethylene. At later times, for example at $10 \mathrm{~ms}$, small amount of ethylene reacts with methyl radical resulting in the formation of vinyl, and over $70 \%$ of vinyl is converted to acetylene. 2-Butene reacts with hydrogen and methyl radical to from $\mathrm{C}_{4} \mathrm{H}_{7}$, and 
$80 \%$ of it is consumed to produce 1,3-butadiene. Again, the stable species have been quantified in the MFTR experiments and is consistent with shock tube data reported in Pyun et al. [58]

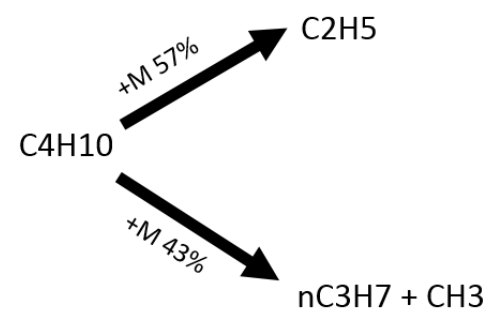

Figure 3.38: Reaction path flux analysis for $\mathrm{C}_{4} \mathrm{H}_{10}$ pyrolysis at $\mathrm{p}=1 \mathrm{~atm}, \mathrm{~T}=1100 \mathrm{~K}$ and 4 ns.

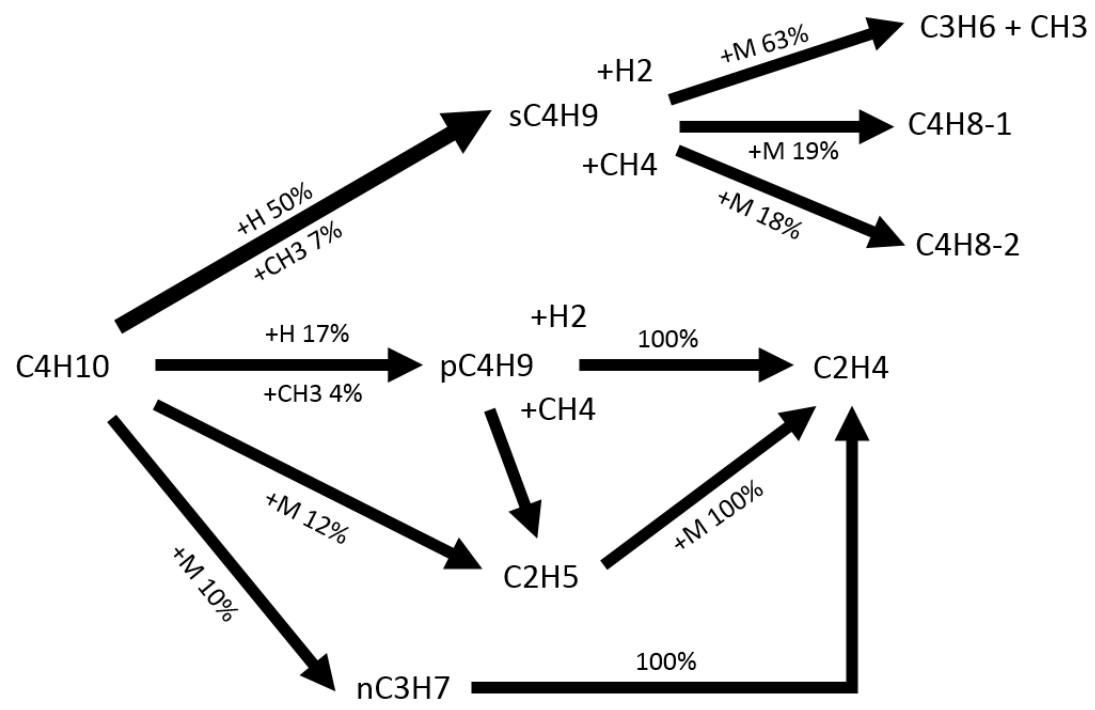

Figure 3.39: Reaction path flux analysis for $\mathrm{C}_{4} \mathrm{H}_{10}$ pyrolysis at $\mathrm{p}=1 \mathrm{~atm}, \mathrm{~T}=1100 \mathrm{~K}$ and $0.1 \mathrm{~ms}$.

Interestingly, the sensitivity analysis of n-butane pyrolysis shown in Fig. 3.37 indicates that at $50 \mathrm{~ms}$ the two initiation reactions are the top two sensitive reactions, followed by the hydrogen abstraction reaction by H-atoms. The uncertainty factor of these reactions vary between 2 and 3, implying that the most sensitive reactions and the important reaction pathways in the modeling of n-butane pyrolysis have large 


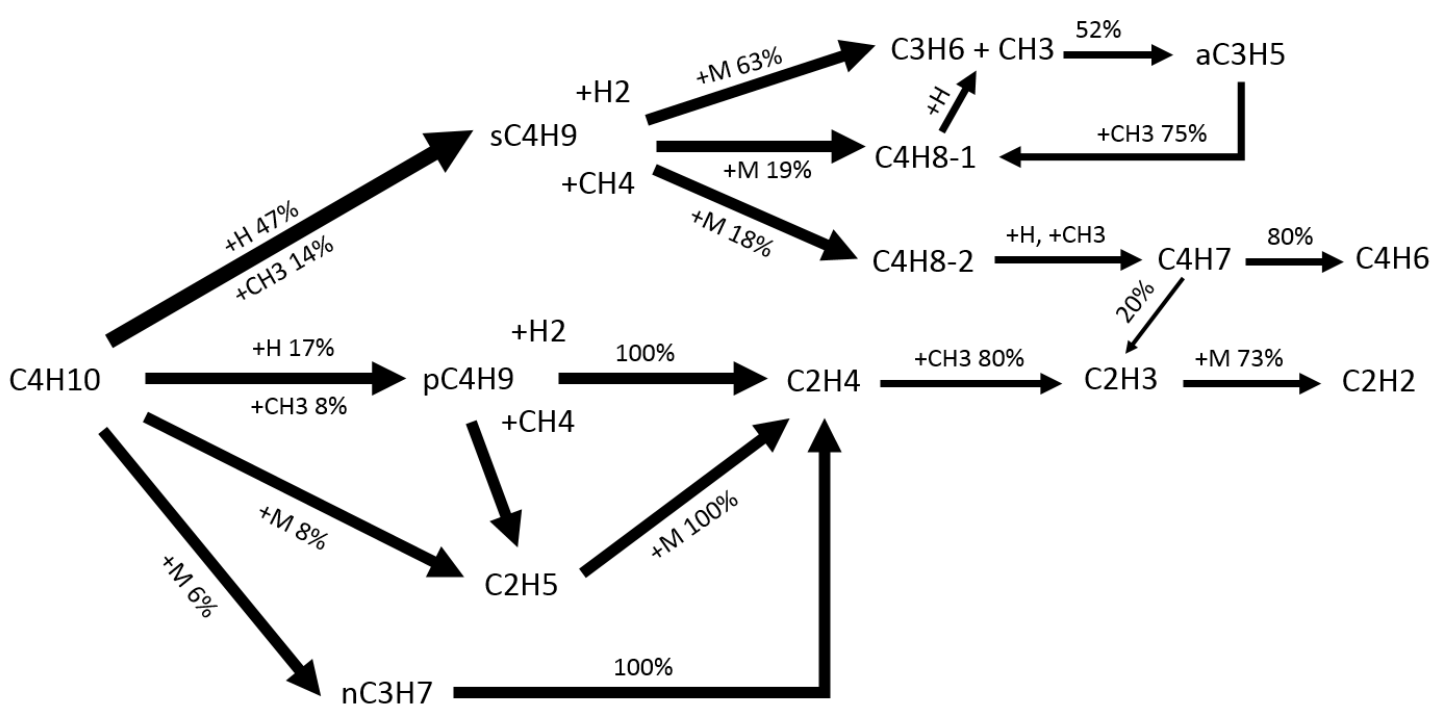

Figure 3.40: Reaction path flux analysis for $\mathrm{C}_{4} \mathrm{H}_{10}$ pyrolysis at $\mathrm{p}=1$ atm, $\mathrm{T}=1100 \mathrm{~K}$ and $10 \mathrm{~ms}$.

uncertainties. In the following section, the effect of chemical kinetic parameter uncertainties on species model prediction are compared with the experimental measurement uncertainties.

Figures 3.41-3.49 show the Monte Carlo simulation of the n-butane pyrolysis at atmospheric pressure and $1100 \mathrm{~K}$ condition.

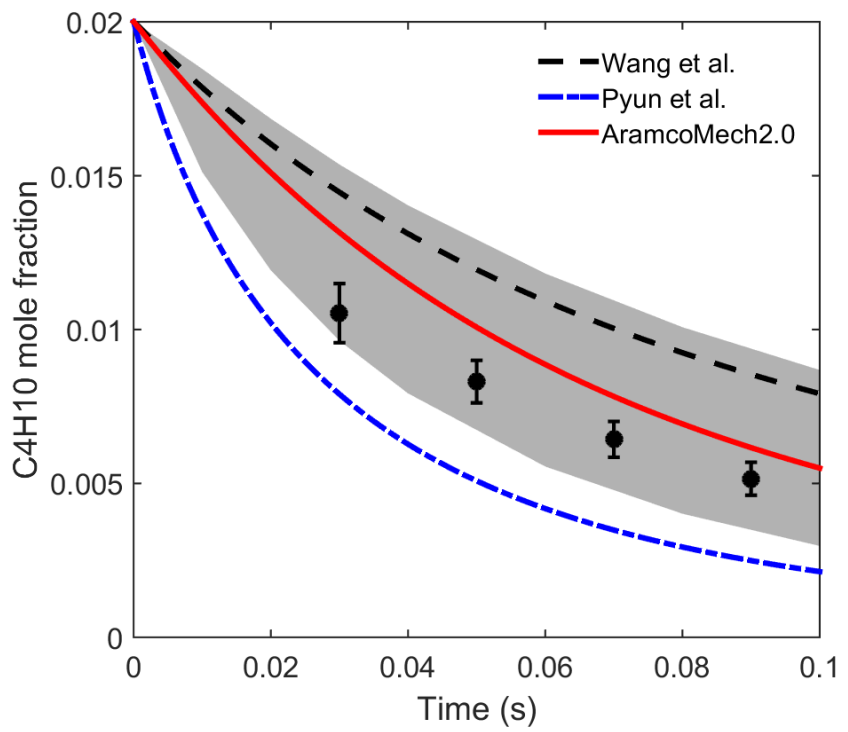

Figure 3.41: Mole fraction as a function of temperature 


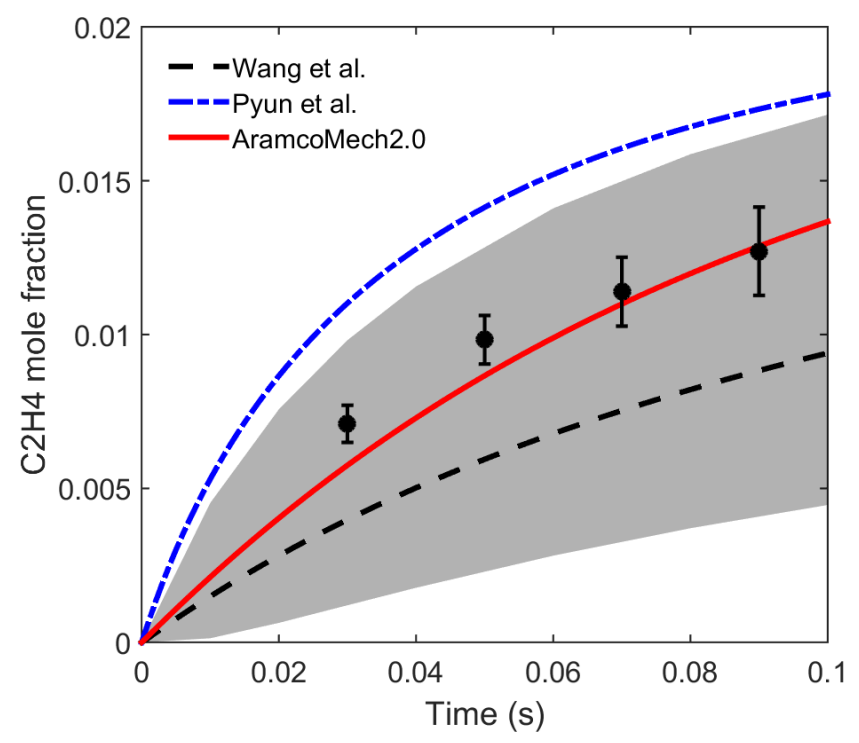

Figure 3.42: Mole fraction as a function of temperature

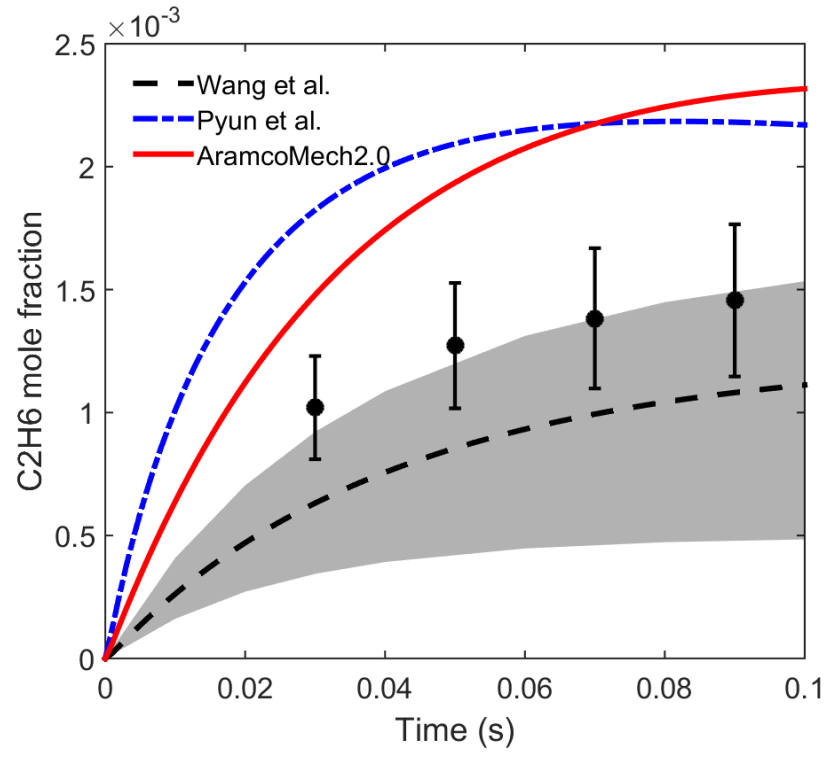

Figure 3.43: Mole fraction as a function of temperature 


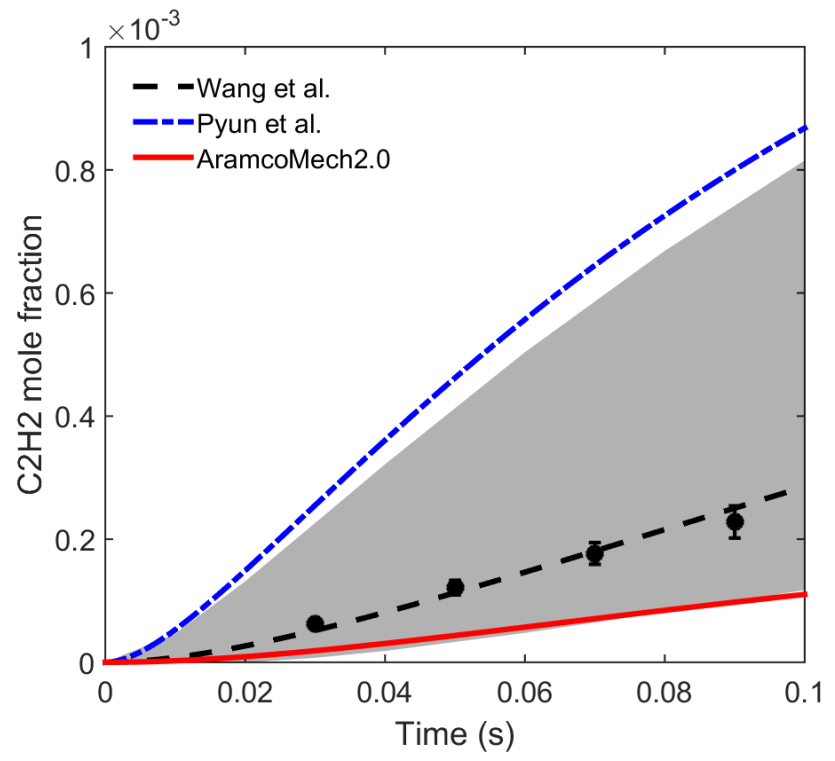

Figure 3.44: Mole fraction as a function of temperature

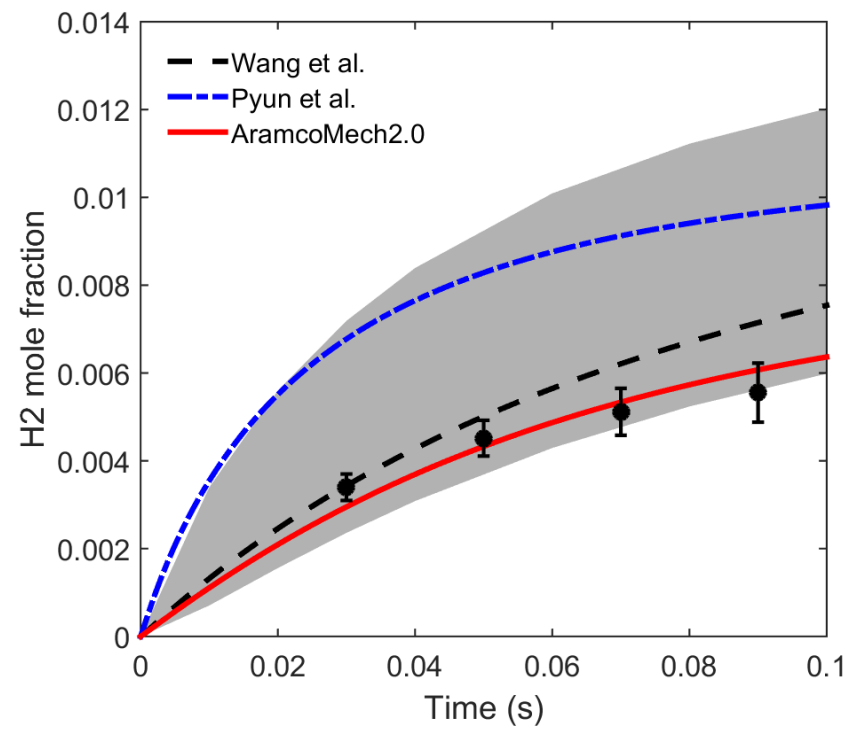

Figure 3.45: Mole fraction as a function of temperature 


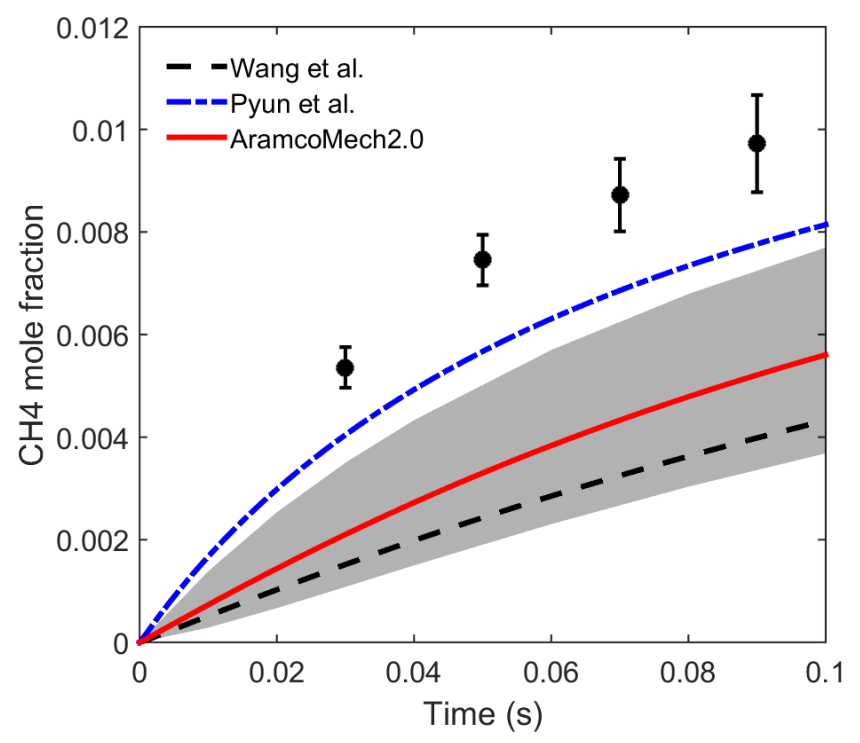

Figure 3.46: Mole fraction as a function of temperature

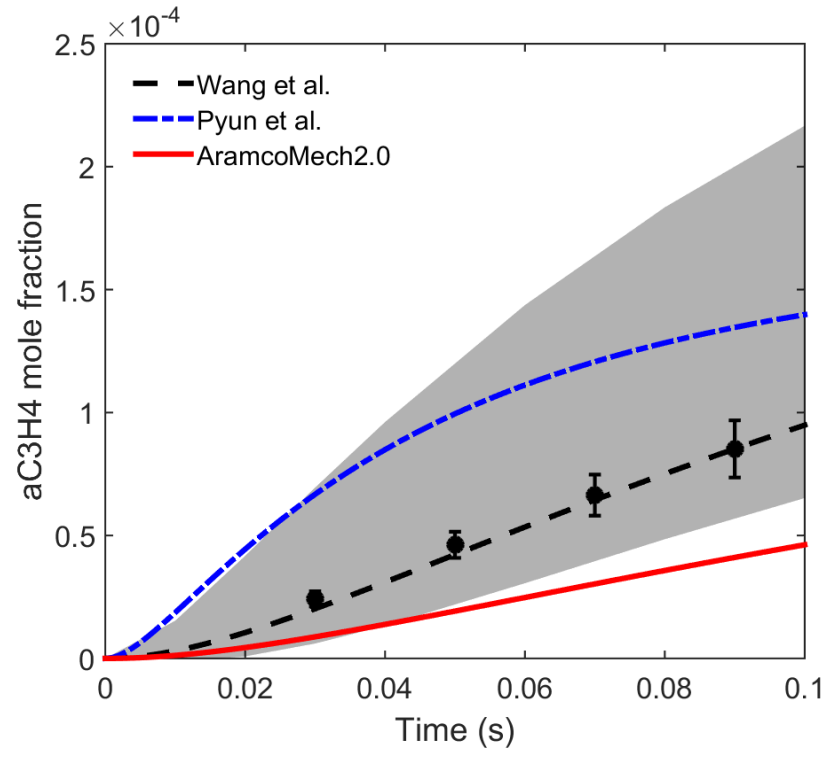

Figure 3.47: Mole fraction as a function of temperature 


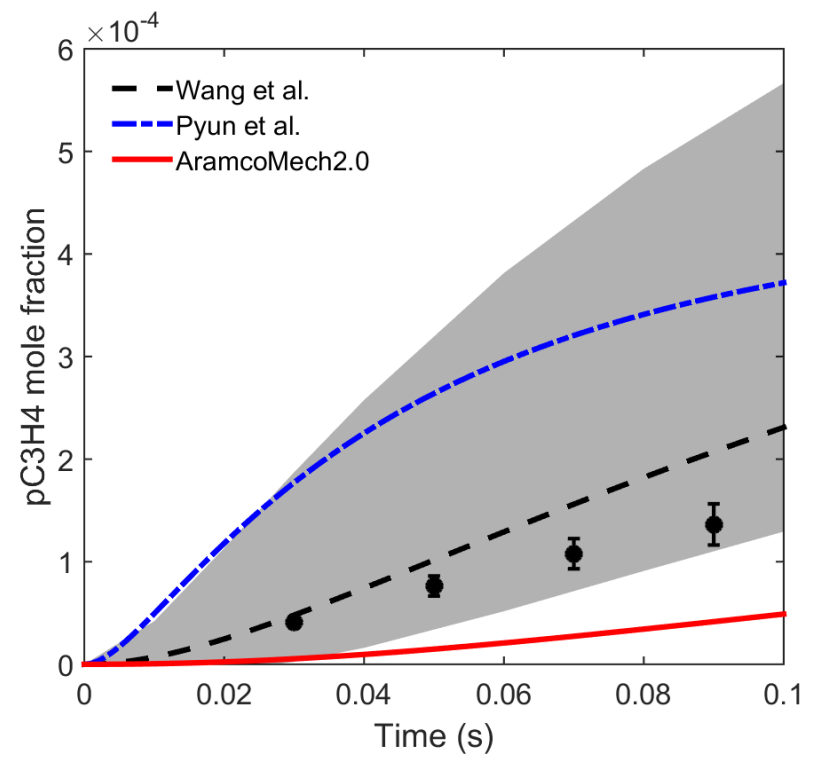

Figure 3.48: Mole fraction as a function of temperature

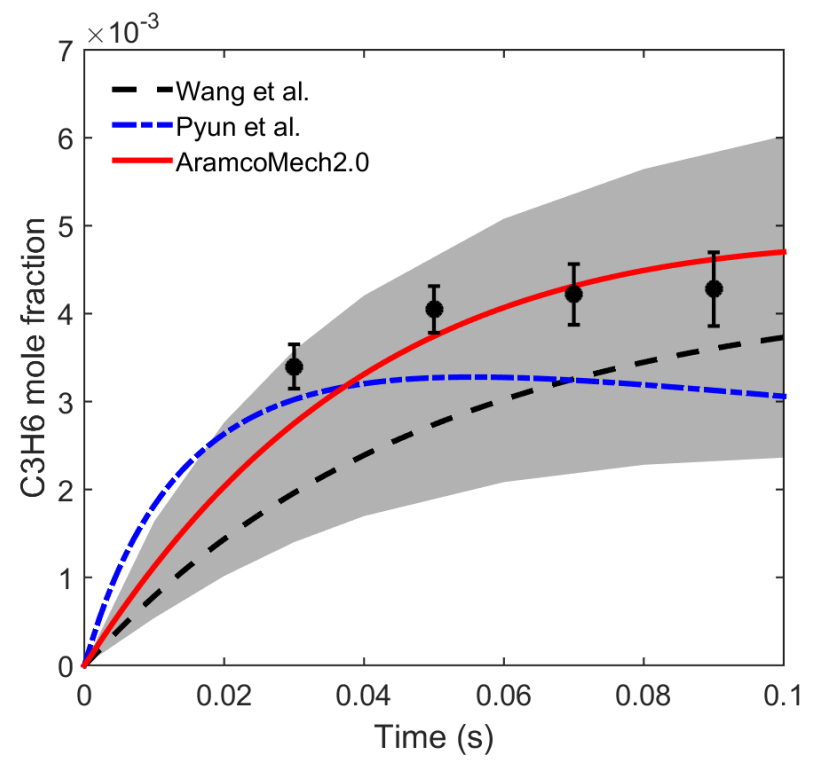

Figure 3.49: Mole fraction as a function of temperature

\subsection{Summary}

A complete sensitivity analysis and uncertainty quantification was performed for ndodecane, ethane, and n-butane pyrolysis. Sensitivity analysis and reaction pathway analysis were performed to identify the important reactions contributing to each fuel 
pyrolysis pathways and governing the major species mole fractions at the corresponding conditions. Based on these findings, a computationally expensive Monte Carlo simulation with a a large sample size was performed in order to quantify the model uncertainty. The results were compared with the experimental data and measurements uncertainty. Results showed that the uncertainties from the chemical models are much higher than the experimental uncertainties. Thus, chemical kinetic model optimization are needed in order to reduce the uncertainties associated with the existing models. For example, acetylene formation predictions from n-dodenace pyrolysis were studied between the four models and the reason of the significant discrepancy was explored and identified for future model optimization purposes.

Furthermore, based on the analysis performed in this chapter, a reduced reaction mechanism was developed for n-dodecane pyrolysis to be used in multi-dimensional reacting flow simulation. The predictions from the reduced model were compared with the detailed model and showed less than $2 \%$ error for models with 50 species or more. 


\section{Chapter 4}

\section{Two-Dimensional Axisymmetric Simulation of a Tubular Cooling}

\section{Channel}

As previously mentioned, tubular flow reactors are simulated with simple ideal plug flow model in the combustion community. This ideal assumption is not true in real experimental condition. The main parameters that can effect this assumption and introduce departure from idealized plug flow assumption, are the initial and boundary conditions.

For laminar flows in tube reactor (Reynolds number is less than 1000 for the experimental conditions explored in this study), the velocity profile is parabolic. This is the main deference with the ideal plug flow with flat velocity profile. This radial gradient in the flow velocity causes residence time stratification in different layers of the tube reactor. As a result the ideal assumption of radially uniform species mole fraction profile is no longer valid. Also the realistic transport properties of the species are far different than what is assumed in plug flow assumption.

The second non-ideality of a real laminar tube reactor is the temperature profile 
inside the flow. The temperature of the tube reactor is controlled by thermocouples on the tube wall. The PID controlled heaters keep the tube wall temperature at a constant set value. This does not necessarily mean the temperature profile of the flow inside the reactor is constant.

To investigate all these non-ideal effects in a laminar tube reactor, a two-dimensional simulation is performed including chemical kinetics and realistic transport properties.

Figure 4.1 shows an illustration of an axi-symmetric parabolic velocity profile in a tube reactor. The velocity gradient in the radial direction causes a radially non-uniform species profile. The fluid element closer to the tube wall has a slower velocity hence a longer residence time compared to the fluid element in the center line. Therefore, the reaction progress is faster at the boundary than at the center-line axis of the tube.

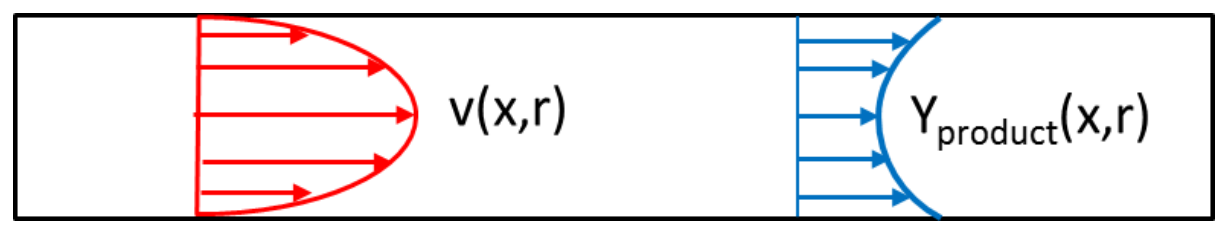

Figure 4.1: Schematic of a parabolic flow reactor with species profiles as a function of the axial and radial coordinate.

\subsection{Two-dimensional Model Governing Equations}

An open-source computational fluid dynamics package, OpenFOAM (Open Field Operation and Manipulation), is used in this work to perform the multi-dimensional reacting flow simulation of the tube reactor $[94,95]$.

The governing equations for the conservation of mass, momentum, species, and energy are [96]: 


$$
\begin{aligned}
\frac{\partial \rho}{\partial t}+\nabla \cdot(\rho \vec{v}) & =0 \\
\frac{\partial \rho \vec{v}}{\partial t}+\nabla \cdot(\rho \vec{v} \vec{v}) & =-\nabla p+\nabla \cdot \mathbf{T}+\rho \vec{g} \\
\frac{\partial \rho Y_{k}}{\partial t}+\nabla \cdot \rho \vec{v} Y_{k} & =-\nabla \cdot\left(\rho Y_{k} \vec{V}_{k}\right)+\dot{\omega}_{k} W_{k}, \quad k=1, \ldots, K \\
\frac{\partial \rho h}{\partial t}+\nabla \cdot \rho \vec{v} h & =\nabla \cdot(\lambda \nabla T)-\sum_{k=1}^{K} \rho Y_{k} \vec{V}_{k} c_{p, k} \nabla T-\sum_{k=1}^{K} h_{k} \dot{\omega}_{k} W_{k}
\end{aligned}
$$

where $t$ is the time, $\rho$ is the mixture density, $p$ is the pressure, $\vec{v}$ is the mixture velocity vector, $\mathbf{T}$ is the deviatoric stress tensor, $\vec{g}$ is the gravity vector, $Y_{k}$ is the mass fraction of species $k, \vec{V}_{k}$ is the diffusion velocity of species $k, \dot{\omega}_{k}$ is the molar production rate of species $k$ per unit volume by chemical reaction, $T$ is the temperature, $c_{p}$ and $c_{p, k}$ are the specific heat at constant pressure of the mixture and of species k, respectively, $\lambda$ the thermal conductivity of the mixture; $h$ and $h_{k}$ are the mixture enthalpy and the individual-species enthalpy, respectively.

The diffusion velocities are calculated by Fick's law of diffusion:

$$
\overrightarrow{V_{k}}=-\frac{D_{k}}{X_{k}} \nabla X_{k}
$$

where $D_{k}$ is the individual-species mixture-averaged diffusion coefficient which is given in terms of binary diffusion coefficients $D_{k j}$ by

$$
D_{k}=\frac{1-Y_{k}}{\sum_{j \neq k}^{N} \frac{X_{j}}{D_{k j}}}
$$

The conservation equations are solved in a segregated manner using second-order accurate total variation diminishing (TVD) Van-Leer schemes. The finite volume equations are integrated in time using the first-order implicit, Eulerian method. Each equation is solved by iterating until the L2-norm of the residual to all equations fell 
below $10^{-7}$. Simulations are run for a specific number of time integrations until steady state is reached and confirmed by monitoring solution residuals.

\subsection{Geometry and Grid Resolution}

As OpenFOAM solver operates in a three dimensional Cartesian coordinate system, all geometries are required in three dimensions. In order to solve cases with less than three dimensions, special boundary condition must be used on the planes normal to the direction of interest, i.e. 'empty' for a two-dimensional case and 'wedge' for an axisymmetric problem. Figure 4.2 illustrates the geometry used for the two-dimensional axi-symmetric problem. The wedge angle must be less than $5^{\circ}$ and the top of the wedge is considered the tube wall.

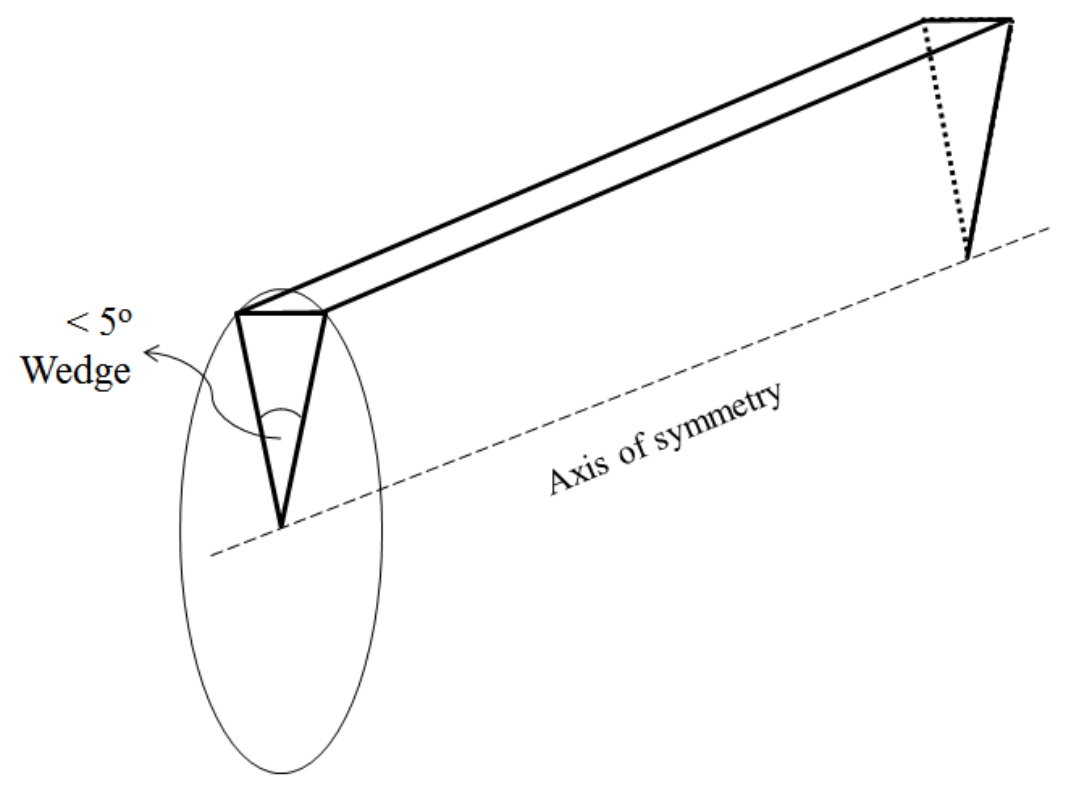

Figure 4.2: Wedge geometry used for axi-symmetric modeling with OpenFOAM solver.

The diameter of the tube is $4 \mathrm{~mm}$ and the length of the tube $40 \mathrm{~cm}$. The grid resolution used is 400 by 100 grid points in axial and radial direction, respectively. Grid convergence has been confirmed by performing two additional cases with coarser 
mesh.

\subsection{Boundary and Initial Conditions}

The governing equations require boundary conditions to be specified for pressure, temperature, velocity, and species mass fraction. The pressure value is fixed at $1 \mathrm{~atm}$ at the outlet boundaries and a zero-gradient condition is imposed at the inlets and at the walls. The temperature is fixed at the inlet and at the wall to the experimental temperature condition and zero-gradient for the outlet. For this simulation the fixed wall temperature is $1100 \mathrm{~K}$. For the velocity field, no-slip conditions are assumed at the walls. At the inlet of the reactor, a parabolic velocity profile is modeled in a way that the corresponding flow rate is equivalent to plug flow velocity of $4 \mathrm{~m} / \mathrm{s}$. A simulation with plug flow velocity profile at the inlet showed negligible effect, due to the fact that the transitions region for fully developed flow is very short. Zerogradient velocity conditions are imposed at the outlet. Boundary conditions for the gas-phase species mass fractions are fixed to the specified experimental inflow value for the inlet and zero-gradient at the outlet and wall. The composition used in this simulation is $0.25 \%$ n-dodecane diluted in $99.75 \%$ N2.

\subsection{Two-dimensional Axi-symmetric Simulation Re- sult}

\subsubsection{Contour Plots}

Figure 4.3 shows the contour plot of the temperature in the two-dimensional axisymmetric simulation of $\mathrm{n}$-dodecane pyrolysis. The aspect ratio of the axial and radial coordinate is not actual. The radial direction coordinate scale is magnified to improve the visibility of the contour result, since the radius is only $2 \mathrm{~mm}$. The 
temperature of the flow drops only $6 \mathrm{~K}$ to $1094 \mathrm{~K}$ at the beginning of the tube reactor where endothermic chemical reactions are happening at the fastest rate. But due to the constant wall temperature condition and fast thermal transport in the flow, the temperature is recovered and reaches to about $1100 \mathrm{~K}$ in most of the flow field.

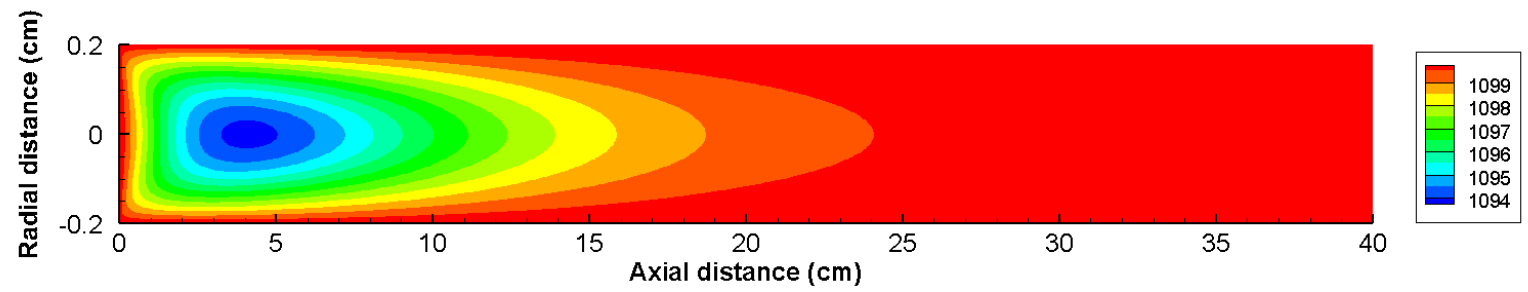

Figure 4.3: The two-dimensional axisymmetric solution contour for temperature $(\mathrm{K})$.

Figures 4.4-4.12 show the contour plot of the major species mole fractions in the two-dimensional axi-symmetric simulation of n-dodecane pyrolysis. The mole fraction of the light weight species (e.g. $\mathrm{H} 2, \mathrm{CH} 4, \mathrm{C} 2 \mathrm{H} 4$ ) have less radial variation compared to the heavier species. The larger molecule species (e.g. n-C12H26, C7H14, C8H16, $\mathrm{C} 10 \mathrm{H} 20, \mathrm{C} 11 \mathrm{H} 22)$ show a large radial variation in the contour plots. The main reason is due to the fact that the diffusion coefficients of these species are substantially larger which makes the diffusion time scale slower compared to the convective time scale. Hence these species produce radially stratified layers along the tube reactor. 


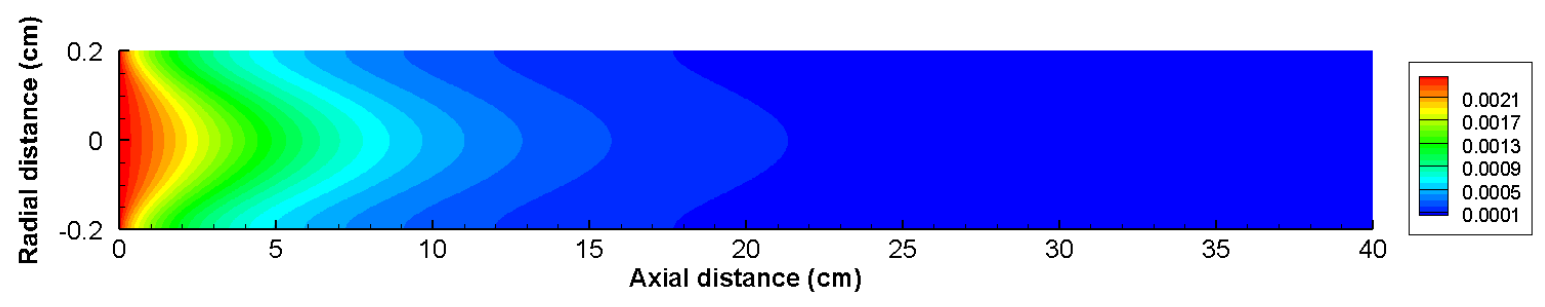

Figure 4.4: The two-dimensional axisymmetric solution contour for n-C12H26 mole fraction.

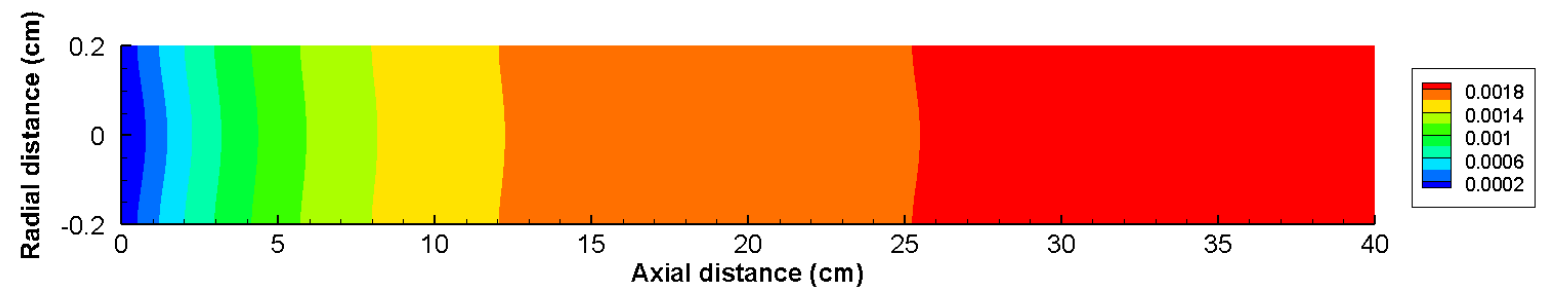

Figure 4.5: The two-dimensional axisymmetric solution contour for $\mathrm{H} 2$ mole fraction.

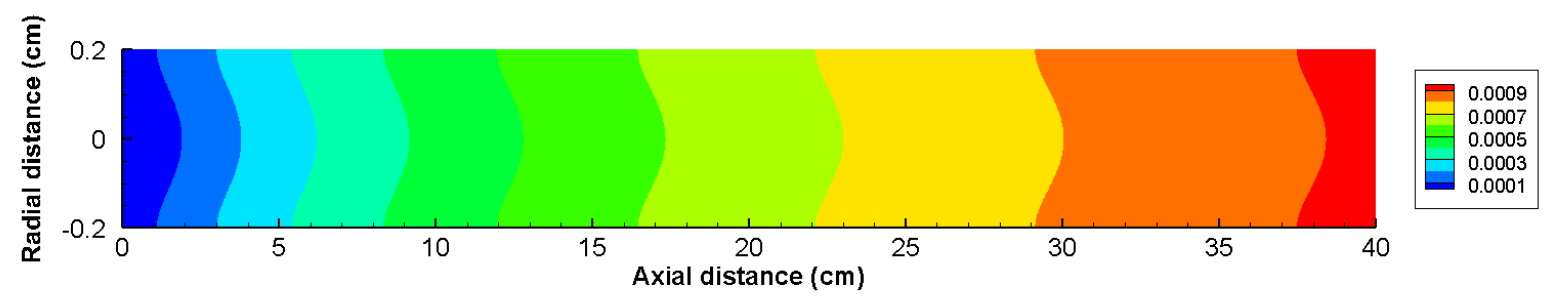

Figure 4.6: The two-dimensional axisymmetric solution contour for $\mathrm{CH} 4$ mole fraction. 


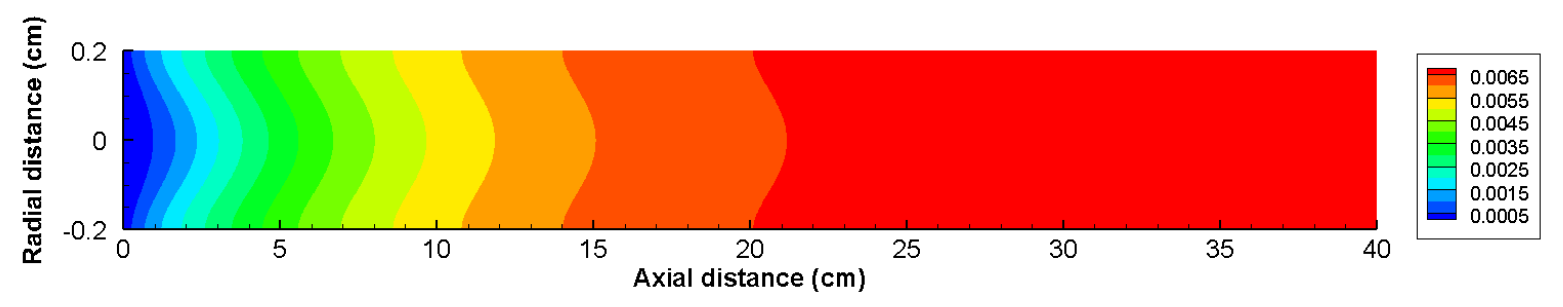

Figure 4.7: The two-dimensional axisymmetric solution contour for $\mathrm{C} 2 \mathrm{H} 4$ mole fraction.

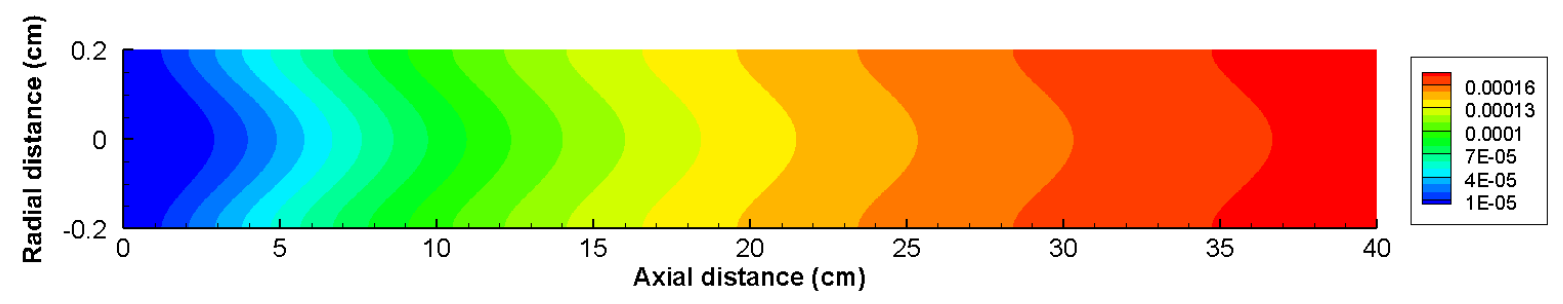

Figure 4.8: The two-dimensional axisymmetric solution contour for $\mathrm{C} 4 \mathrm{H} 6$ mole fraction.

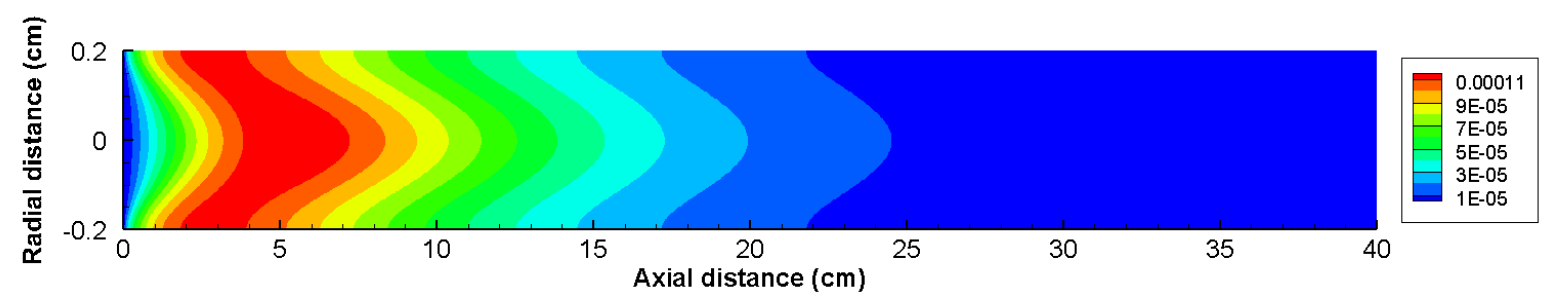

Figure 4.9: The two-dimensional axisymmetric solution contour for C7H14 mole fraction. 


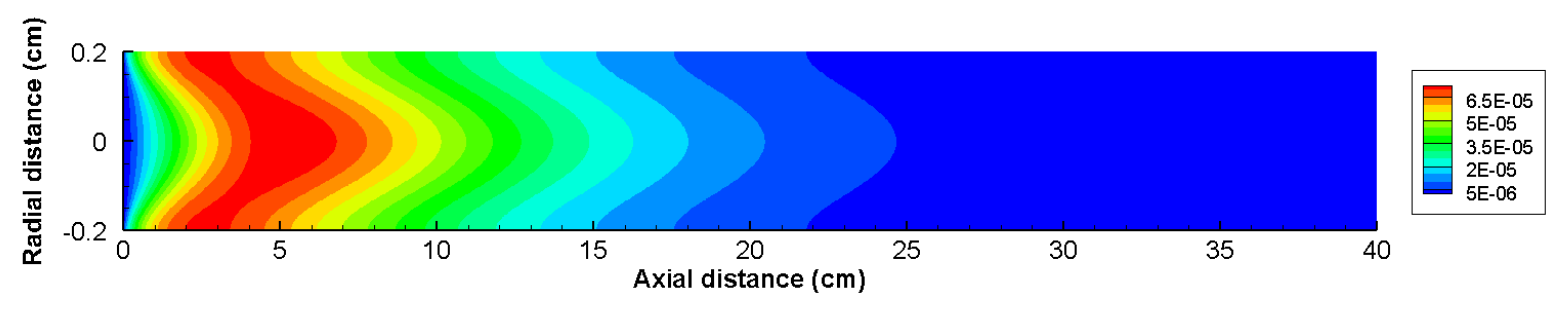

Figure 4.10: The two-dimensional axisymmetric solution contour for C8H16 mole fraction.

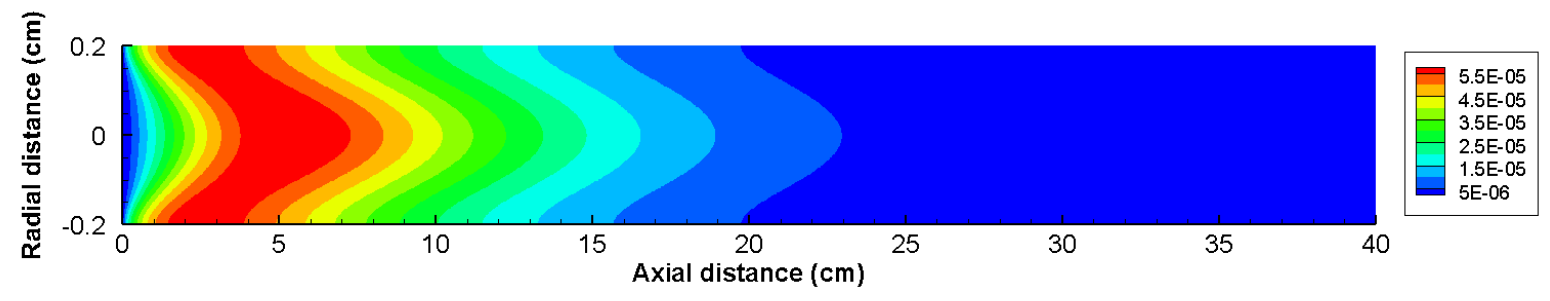

Figure 4.11: The two-dimensional axisymmetric solution contour for C10H20 mole fraction.

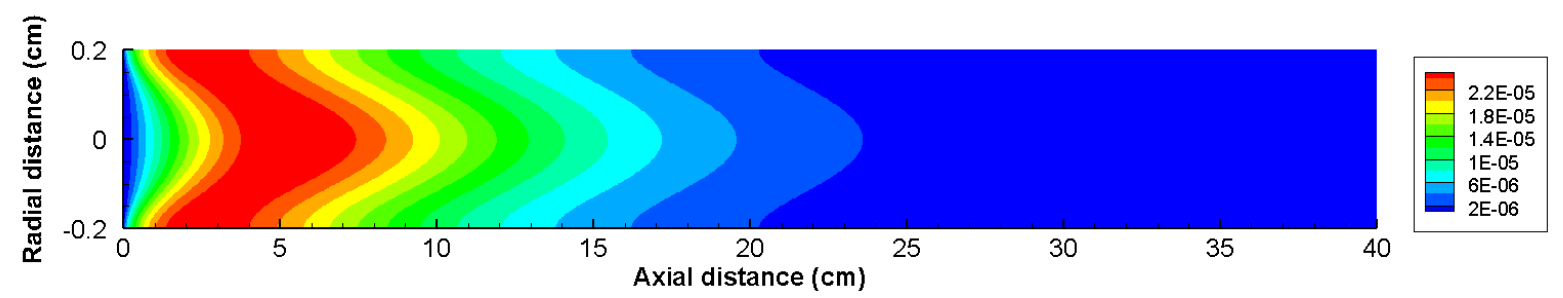

Figure 4.12: The two-dimensional axisymmetric solution contour for $\mathrm{C} 11 \mathrm{H} 22$ mole fraction. 


\subsubsection{Radial Variation Plots}

In order to provide a better analysis of the $2 \mathrm{D}$ axi-symmetric simulation of the $\mathrm{n}$ dodecane pyrolysis, the species mole fractions are plotted along the radius of the tube reactor. Figure 4.13-4.17 compare the species mole fractions across the tube radius at five different axial locations. The figures are based on the axial distances of 1,5 , 8,15 , and $40 \mathrm{~cm}$ along the tube reactor. At each location the mole fraction of all the species are extracted along the radius (the line connecting the center line to the wall of the tube reactor). Then for illustration purposes, the mole fraction of each species is normalized by the maximum amount so the plots are bound to a maximum of 1 for all the species shown in the figures. Also the maximum mole fraction for each species is included in the label of the figures.

At axial location of $1 \mathrm{~cm}$, the radial profile of all the species mole fractions show a significant variation. The fuel conversion is very low at this location (residence time is about $2.5 \mathrm{~ms}$ ). The mole fraction of $\mathrm{n}$-dodecane is maximum at the center line of the tube and about $30 \%$ less at the tube wall. The mole fraction of all the pyrolysis product species are maximum at the wall and minimum at the center line of the tube reactor. All these product species have about $50 \%$ to $90 \%$ mole fraction variation in the radial direction except hydrogen which is about $20 \%$. 

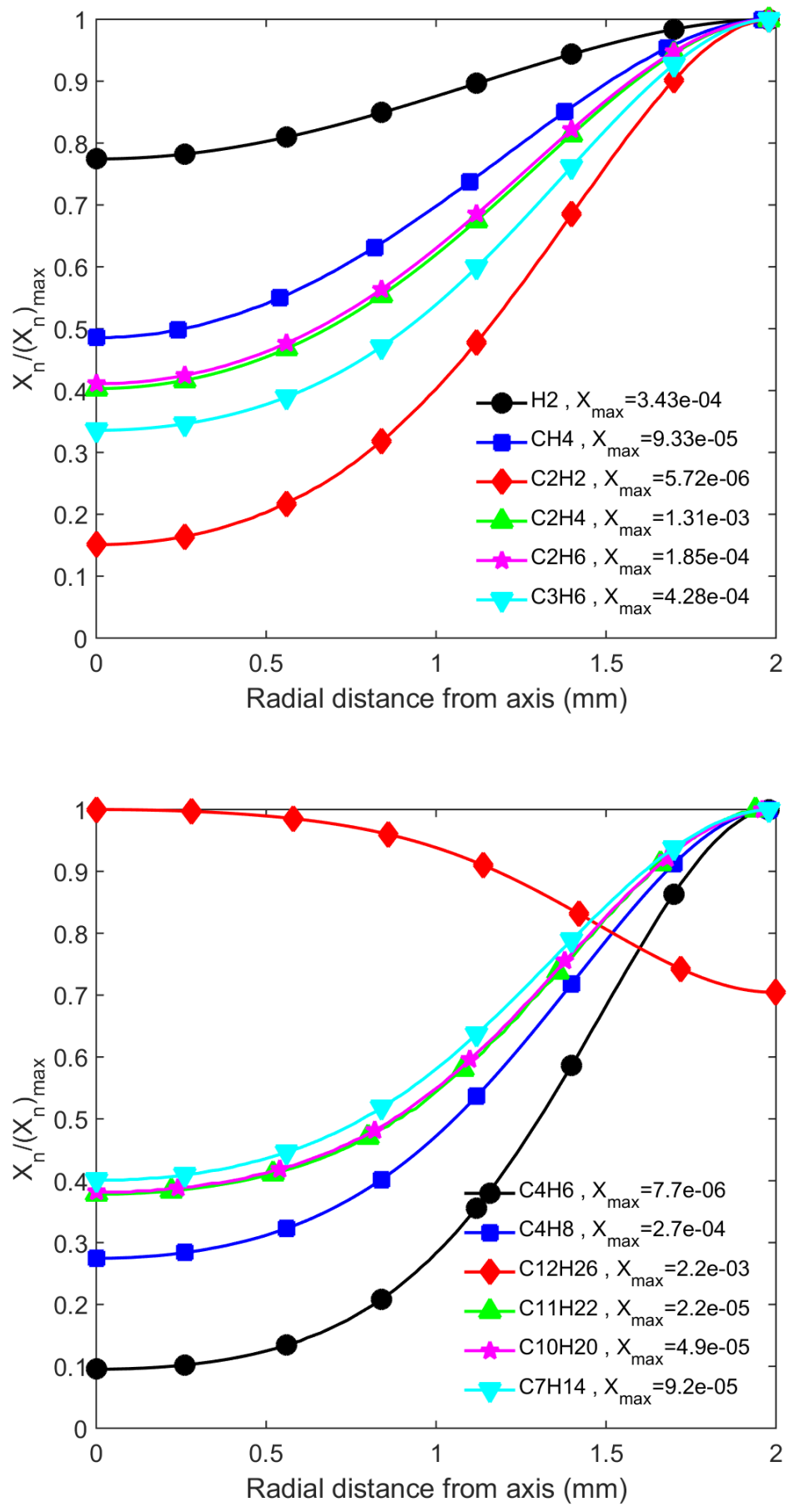

Figure 4.13: Species normalized mole fraction profile across the radial coordinate at $\mathrm{x}=1 \mathrm{~cm}$ from the reactor inflow.

As the axial distance changes towards more downstream of the flow in the tube reactor, the radial variation of the species mole fraction profile decreases. At $5 \mathrm{~cm}$ 
axial distance of the tube, the radial variation of the lighter species (i.e. H2, C1-C3 species) are less than 30\%. Also the heavier species (C4-C12) show a maximum mole fraction change of $50 \%$ in the radial direction. From the inlet point up to about $8 \mathrm{~cm}$ the $\mathrm{C} 7-\mathrm{C} 11$ species absolute mole fraction are increasing. But after this point these large species mole fraction start to decrease.

Figures 4.16 and 4.17 show the radial profiles at even more downstream with $15 \mathrm{~cm}$ and at the end of the tube at $40 \mathrm{~cm}$. The radial variation of the smaller species mole fraction decrease to less than $10 \%$ at $15 \mathrm{~cm}$ and eventually become radially uniform at the end of the tube (less than 2\% change). However, the large species (C7-C12) keep the $30 \%$ radial variation along the tube all the way until the tube end point (see Figures 4.16 and 4.17). As stated before, the slow diffusion time scale of these species compared to the convective residence time is the main reason of this radial variation. This radial stratification of species profile could result in the experimental measurement error depending on the radial location of the sampling probe. 

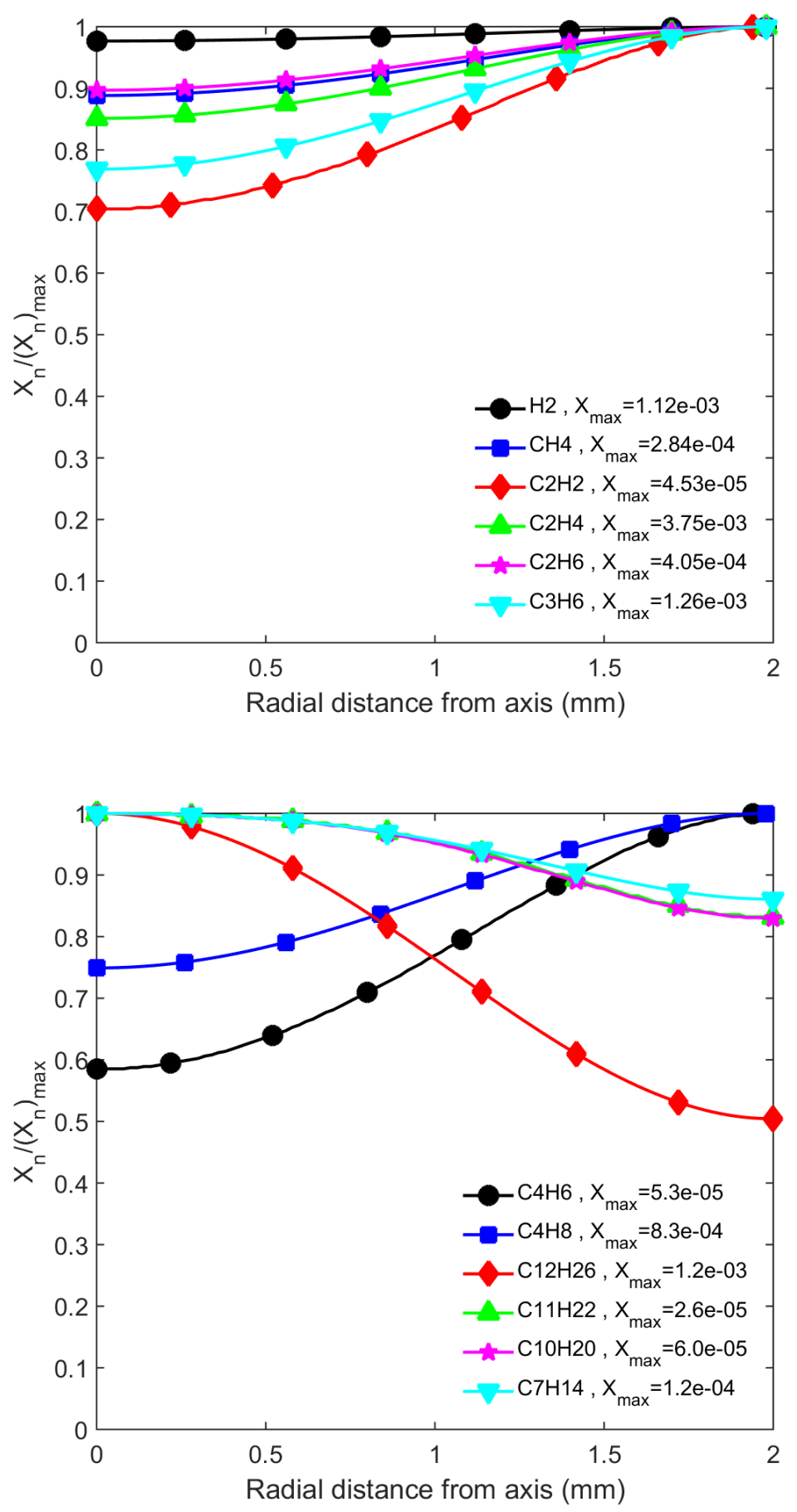

Figure 4.14: Species normalized mole fraction profile across the radial coordinate at $\mathrm{x}=5 \mathrm{~cm}$ from the reactor inflow. 

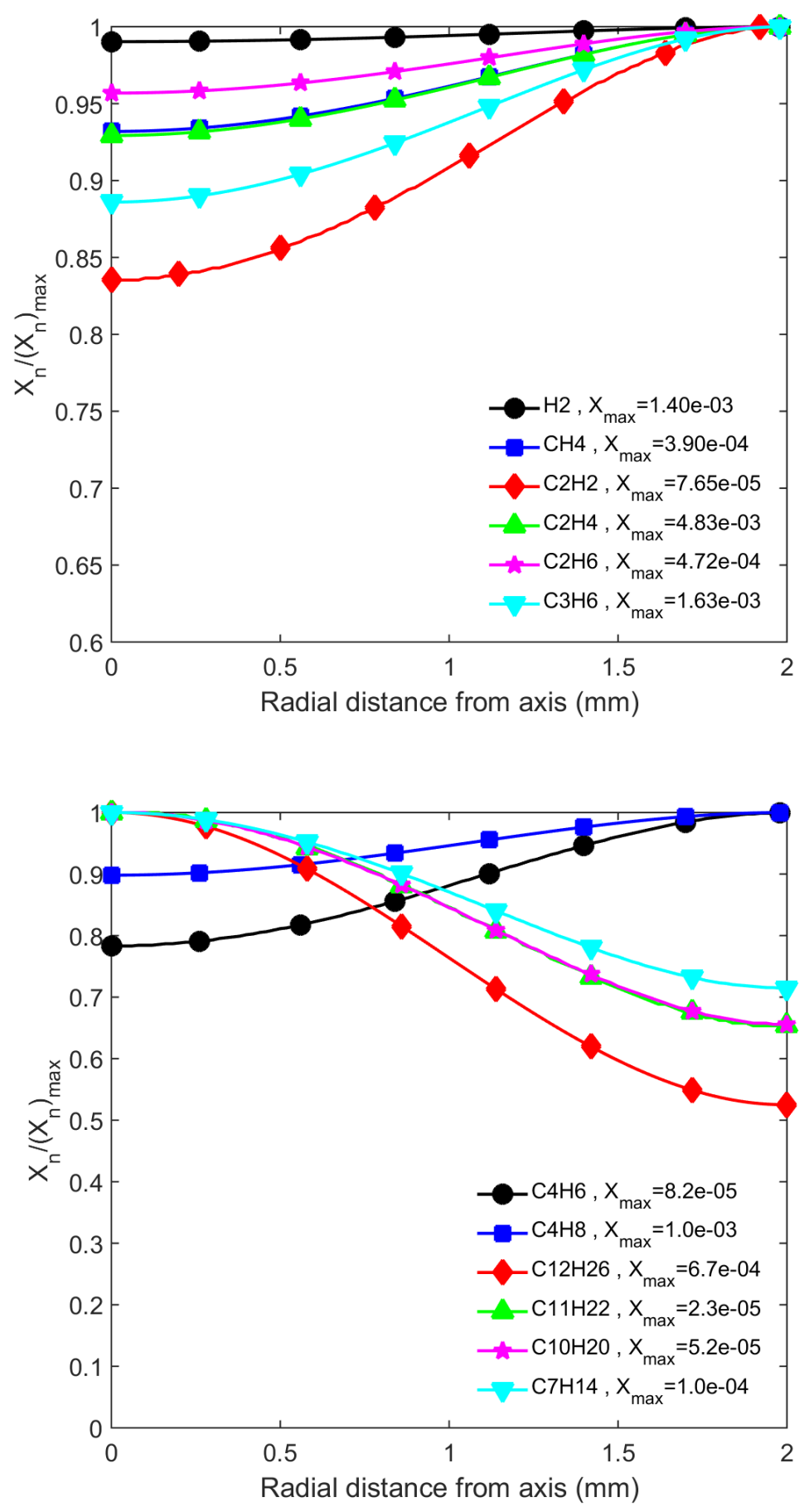

Figure 4.15: Species normalized mole fraction profile across the radial coordinate at $\mathrm{x}=8 \mathrm{~cm}$ from the reactor inflow. 

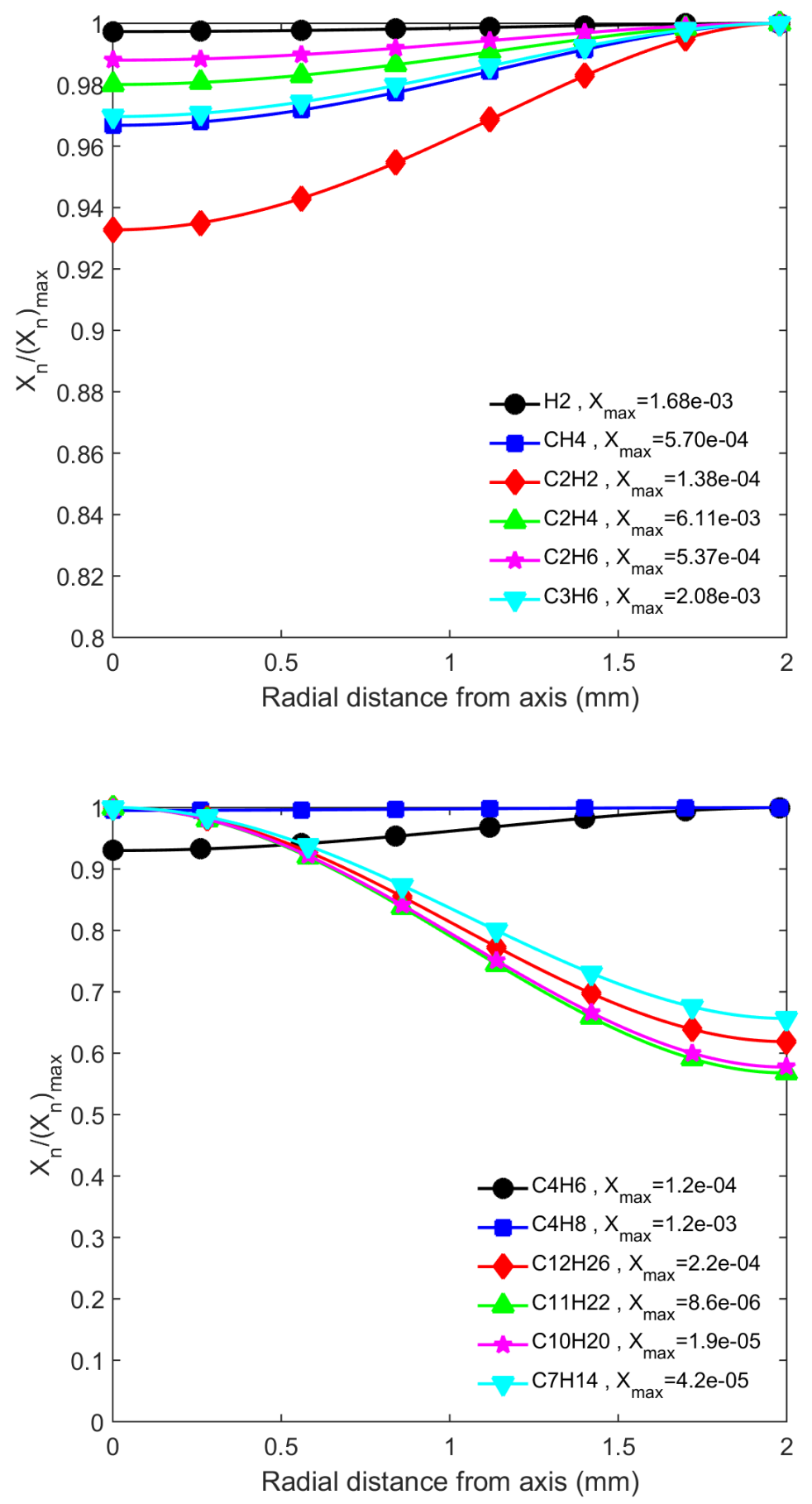

Figure 4.16: Species normalized mole fraction profile across the radial coordinate at $\mathrm{x}=15 \mathrm{~cm}$ from the reactor inflow. 

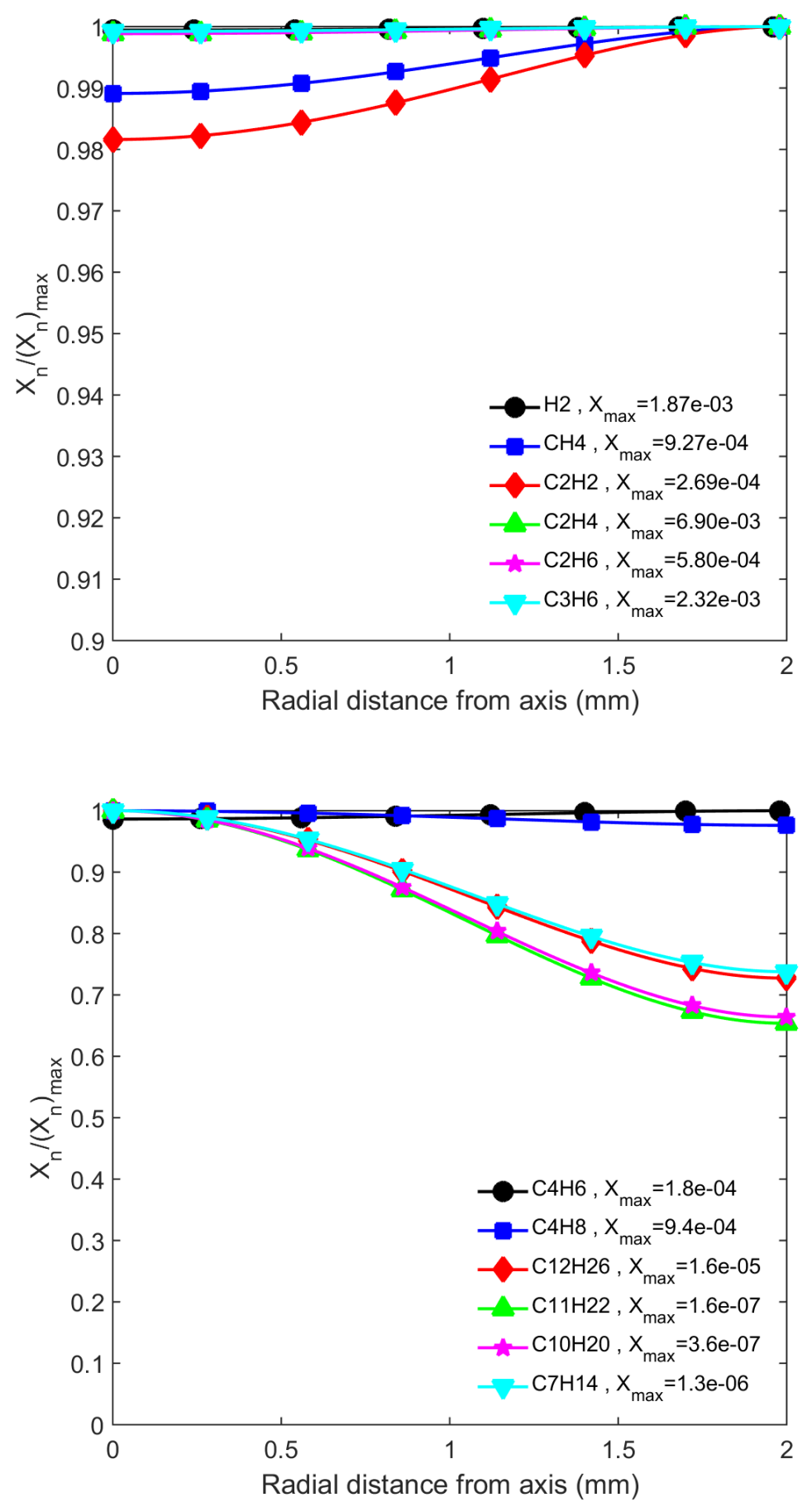

Figure 4.17: Species normalized mole fraction profile across the radial coordinate at $\mathrm{x}=40 \mathrm{~cm}$ from the reactor inflow. 


\subsubsection{Axial Variation Plots}

Figures 4.18 compares the temperature profile along the tube reactor axial direction at two radial locations, tube center-line and near tube wall. Since the wall temperature is kept constant as a boundary condition, the temperature does not change along the tube. However the temperature at the center line drops about $6 \mathrm{~K}$ from the wall temperature at axial location of about $5 \mathrm{~cm}$ where the endothermic effect is maximum. This temperature change is not considered a significant drop since the experimental measurement uncertainty of temperature inside the reactor is about 5 K.

Figures 4.19-4.21 compare different species mole fraction profiles along the tube reactor axial direction between the tube center line and near the tube wall. In the first half of the tube $(0-20 \mathrm{~cm})$ the mole fraction profiles are different between the center line and the wall specially for n-dodecane. After $20 \mathrm{~cm}$ the two profiles merge and the difference becomes negligible. For high diffusive species such as hydrogen the profiles are completely matched.

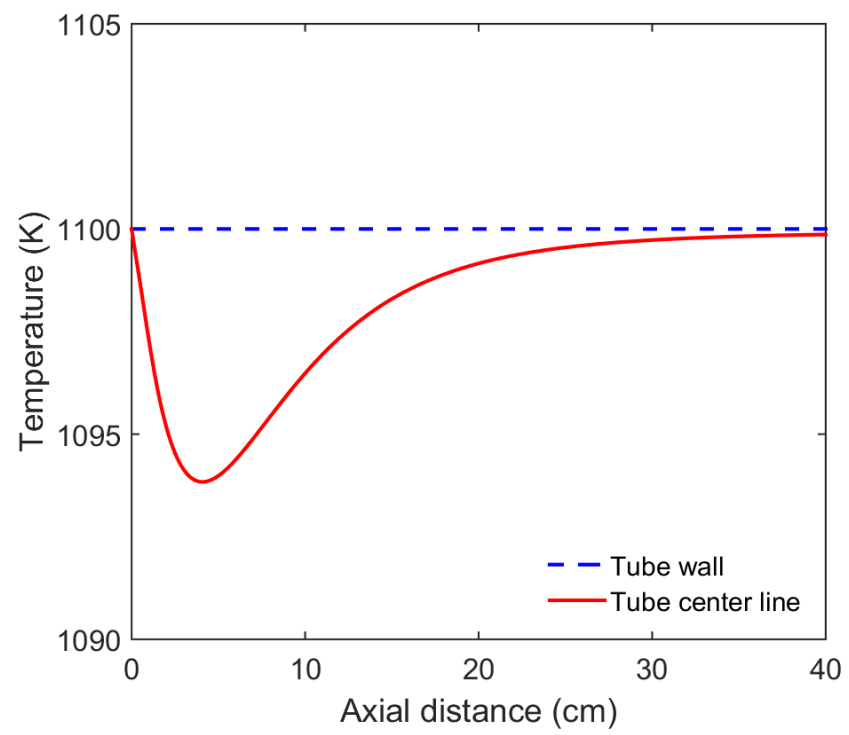

Figure 4.18: Comparison of temperature profiles for the two-dimensional axisymmetric $\mathrm{n}$-dodecane pyrolysis. Solid line is the solution along the center-line axis of the tube and dashed line is the solution at the wall of the reactor. 


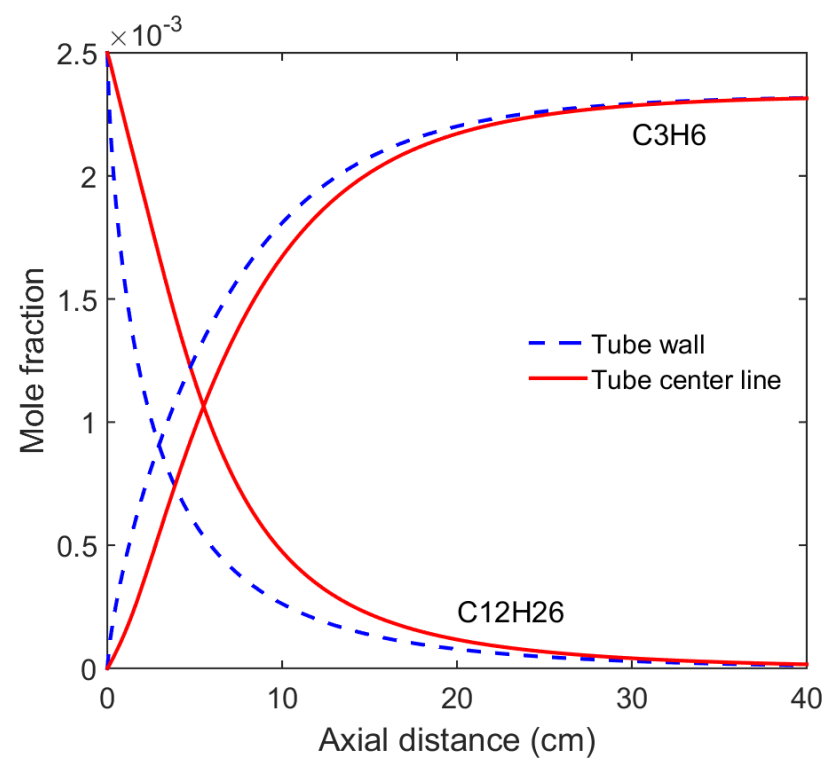

Figure 4.19: Comparison of species mole fraction profiles for the two-dimensional axisymmetric n-dodecane pyrolysis. Solid line is the solution along the center-line axis of the tube and dashed line is the solution at the wall of the reactor.

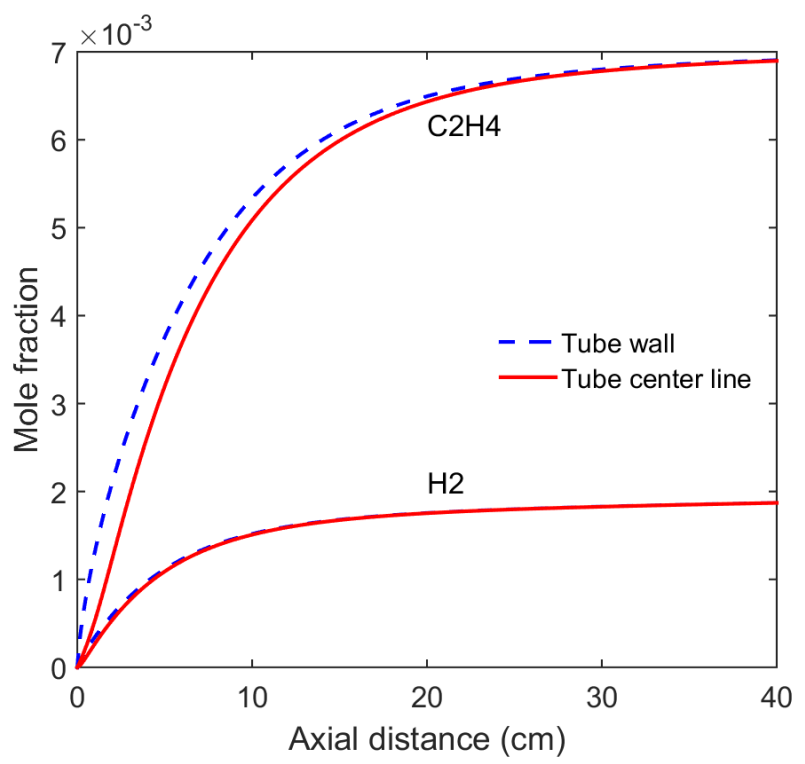

Figure 4.20: Comparison of species mole fraction profiles for the two-dimensional axisymmetric n-dodecane pyrolysis. Solid line is the solution along the center-line axis of the tube and dashed line is the solution at the wall of the reactor. 


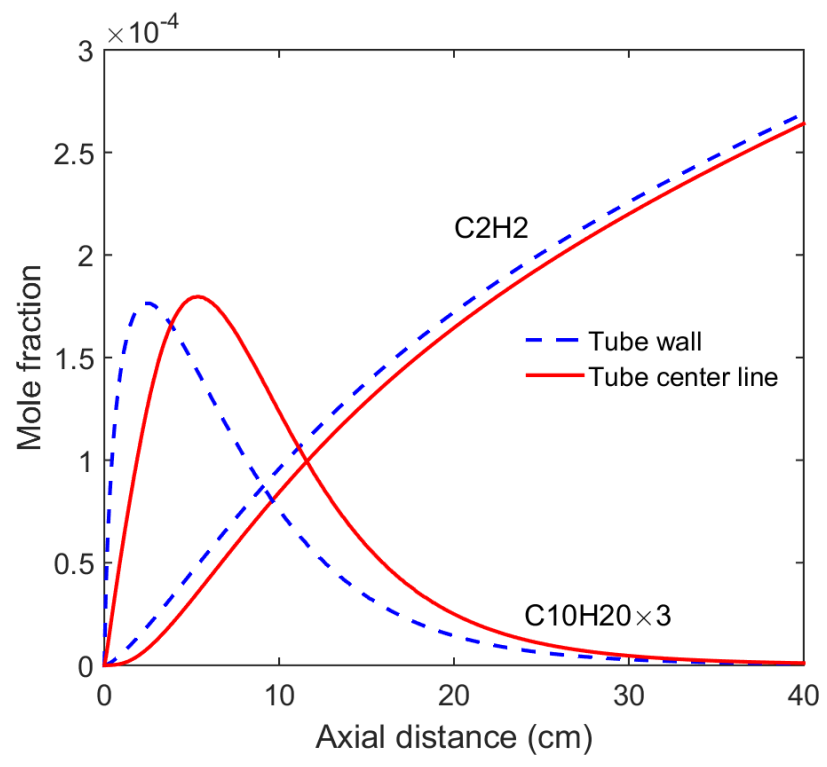

Figure 4.21: Comparison of species mole fraction profiles for the two-dimensional axisymmetric n-dodecane pyrolysis. Solid line is the solution along the center-line axis of the tube and dashed line is the solution at the wall of the reactor.

\subsubsection{Comparison between 2D and 1D}

Here three simulation are performed to model the tube reactor and compared in this section. The zero-dimensional closed adiabatic reactor, the one-dimensional constant temperature reactor, and the two-dimensional axi-symmetric simulation with parabolic velocity profile. The species mole fraction for the $2 \mathrm{D}$ axisymmetric solution are calculated by the cross-sectional averaged values.

Figure 4.22 shows the comparison of the Temperature profiles for n-dodecane pyrolysis in the atmospheric pressure tube reactor for three simulation cases. The zero-dimensional adiabatic solution has a significant temperature drop (30 K) due to the fuel pyrolysis endothermic properties. The one-dimensional case has an imposed constant temperature along the tube reactor. The two-dimensional axi-symmetric simulation with constant wall temperature shows a small temperature drop $(5 \mathrm{~K})$ because of the same endothermic reason. This temperature drop is closer towards the beginning of the tube reactor where the reactions are taking place at the highest 
speed and gradients.

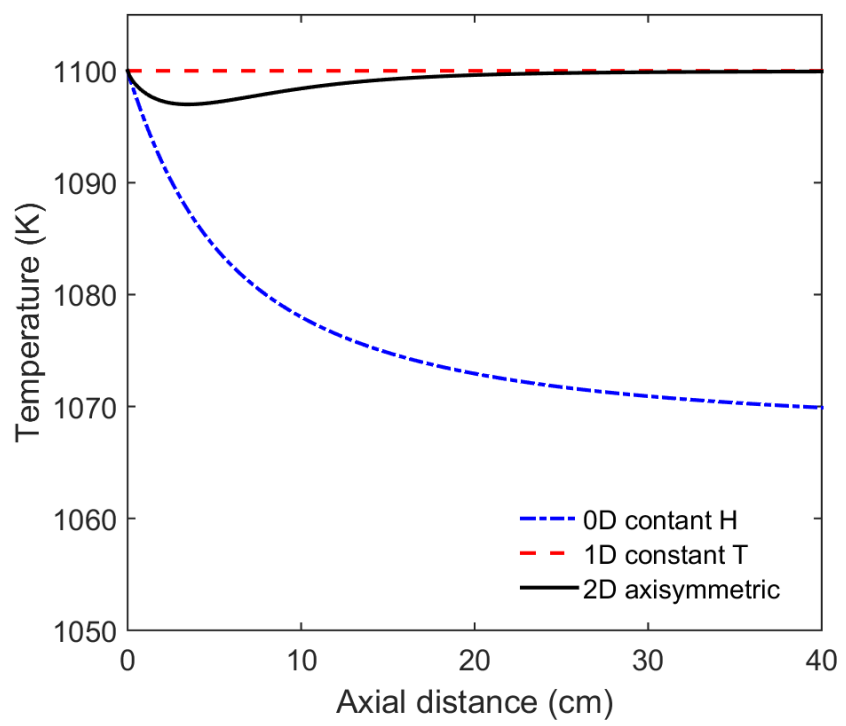

Figure 4.22: Temperature profiles along the tube reactor for n-dodecane pyrolysis. Solid line: 2D axisymmetric solution averaged over the cross-section of the tube reactor. Dashed line: 1D constant temperature solution. Dashed dotted line: 0D constant enthalpy (adiabatic) solution.

Figure 4.23 shows the comparison of the mole fraction profiles for n-dodecane pyrolysis in the atmospheric pressure tube reactor for the three simulation cases. The zero-dimensional adiabatic solution shows less fuel conversion, mainly due to the lower reaction operating temperature. 

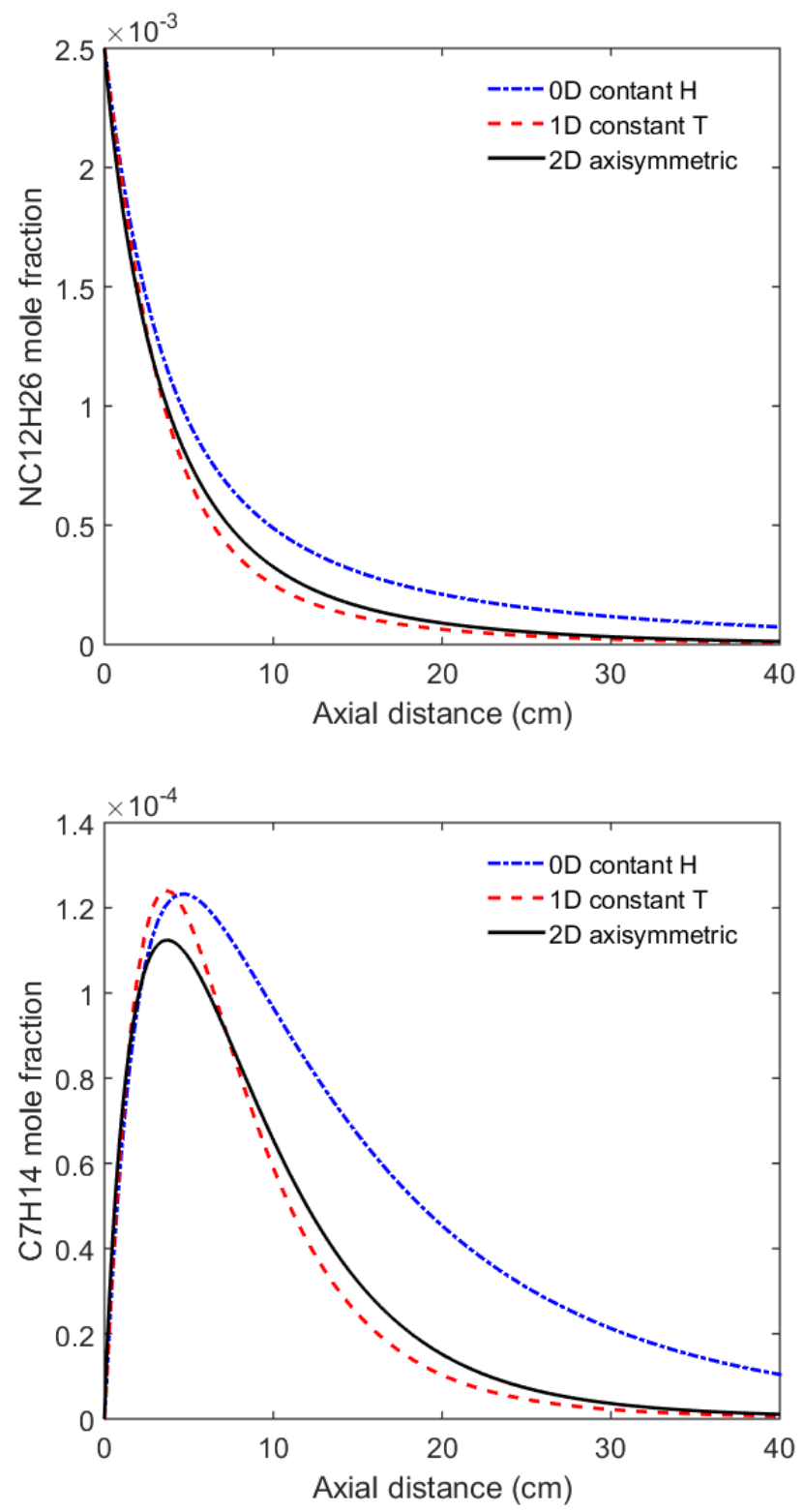

Figure 4.23: Species mole fraction profiles along the tube reactor for $\mathrm{n}$-dodecane pyrolysis. Solid line: 2D axisymmetric solution averaged over the cross-section of the tube reactor. Dashed line: 1D constant temperature solution. Dashed dotted line: 0D constant enthalpy (adiabatic) solution.

The adiabatic solution has shown a large temperature drop compared to the constant temperature case. However, in the experimental reactor the temperature along the tube reactor is controlled by PID controllers and held constant within less than $5 \mathrm{~K}$ from the target temperature. Therefore, the constant temperature condition is closer to the real experimental condition making the adiabatic case unrealistic. From 
this point, the $0 \mathrm{D}$ adiabatic solution is removed from the comparison figure and the analysis focus is on the differences between the $1 \mathrm{D}$ constant temperature solution and 2D axi-symmetric solution with constant wall temperature. Comparing the species mole fraction profiles in Figures 4.24-4.26, the 2D cross-sectional average result is not significantly different than the $1 \mathrm{D}$ constant temperature model result. This is even true for the large species with high radial gradients. As a result, it can be concluded that if the experimental measurement probes a sample from the cross-sectional average of the flow the 1D model could be good approach for even a laminar tube reactor. 

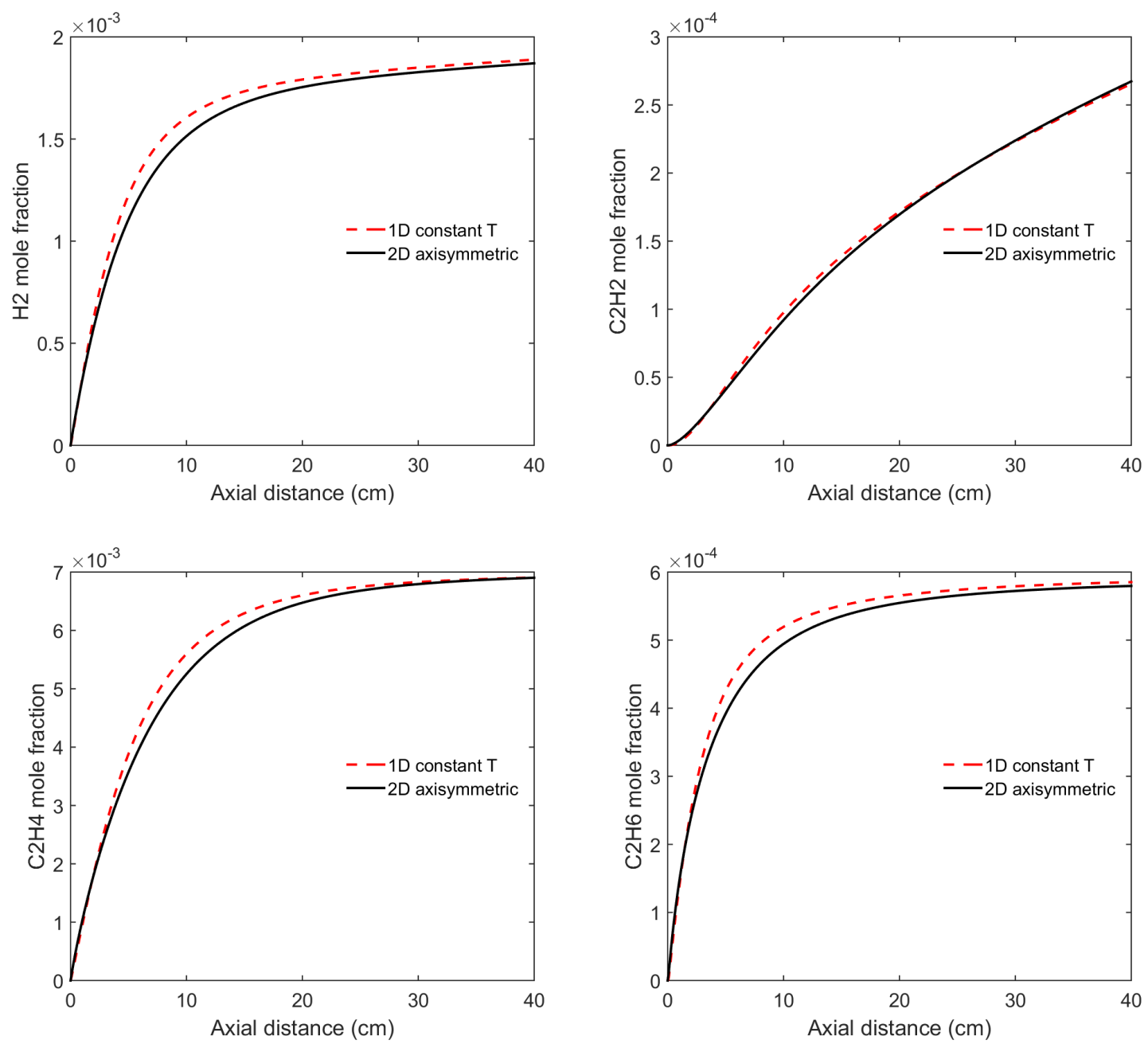

Figure 4.24: Species mole fraction profiles along the tube reactor for n-dodecane pyrolysis. Solid line: 2D axisymmetric solution averaged over the cross-section of the tube reactor. Dashed line: 1D constant temperature solution. 

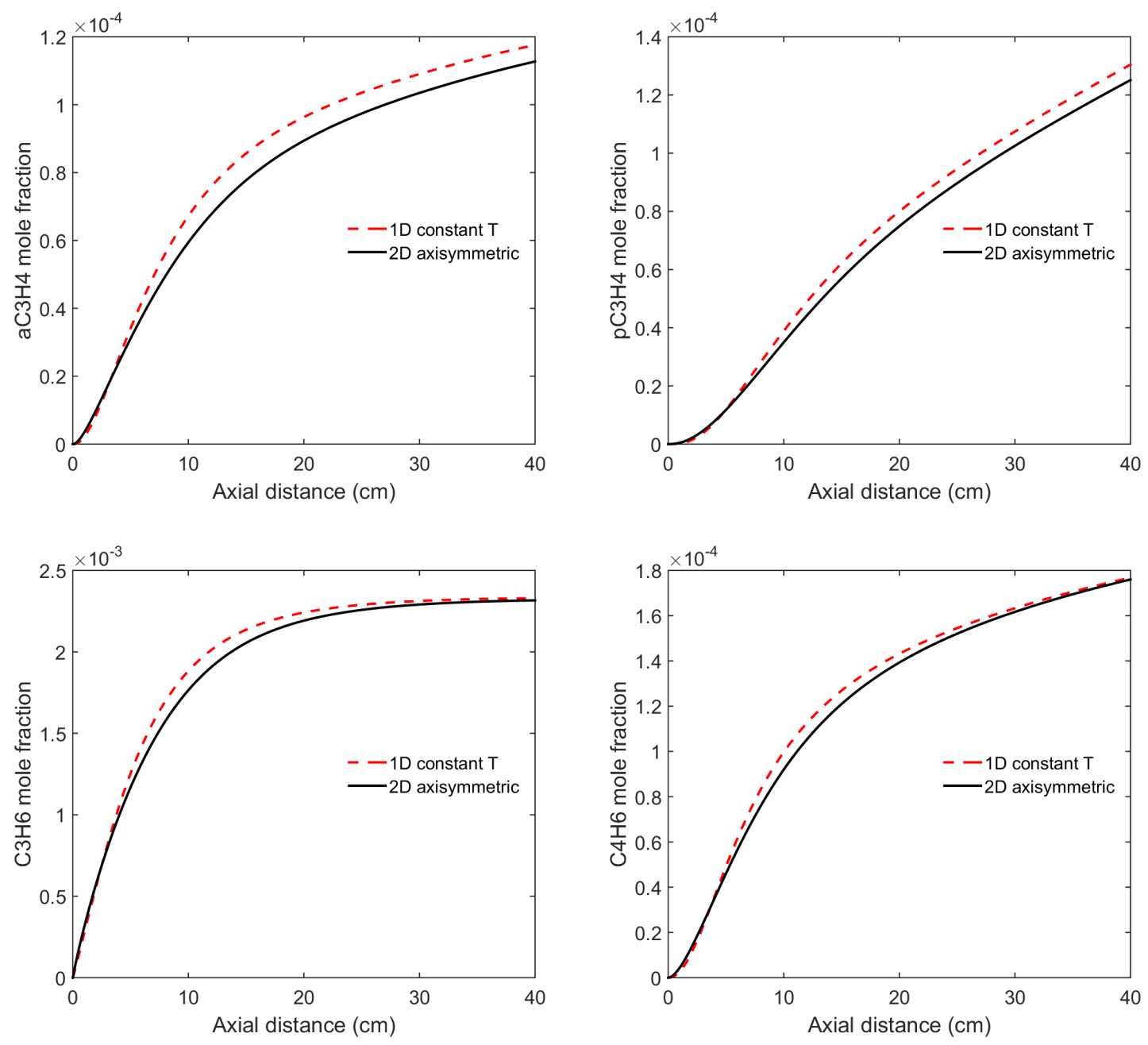

Figure 4.25: Species mole fraction profiles along the tube reactor for $\mathrm{n}$-dodecane pyrolysis. Solid line: 2D axisymmetric solution averaged over the cross-section of the tube reactor. Dashed line: 1D constant temperature solution. 

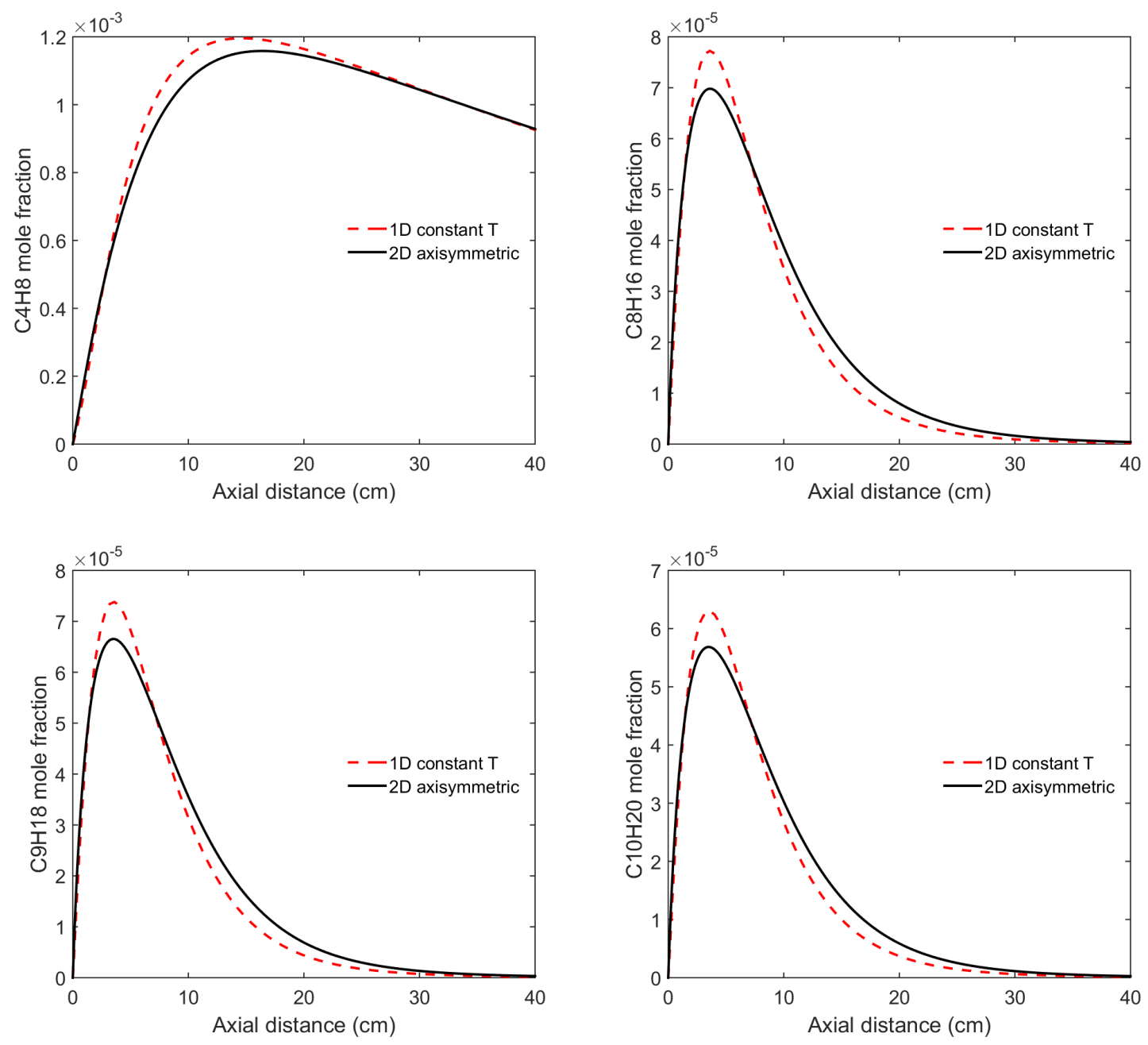

Figure 4.26: Species mole fraction profiles along the tube reactor for n-dodecane pyrolysis. Solid line: 2D axisymmetric solution averaged over the cross-section of the tube reactor. Dashed line: 1D constant temperature solution.

\subsection{Summary}

The two-dimensional effects of the laminar parabolic flow in the tubular reactor were investigated. A complete numerical axi-symmetric Navier-Stokes simulation including chemical reactions was performed in order to understand the stratification issue and radial gradient effects associated with the parabolic velocity profile. The numerical calculation is based on the boundary conditions from the UVa tube reactor 
experiment.

The simulation results showed significant radial variation in the highly reactive region (0-30 ms residence time in the studied case). The radial gradients remain high for the larger species (C7-C12) for the entire region, but the smaller species with faster diffusivity become radially uniform by the end of the reactor. The results from the two-dimensional numerical simulation of n-dodecane pyrolysis were compared with the one-dimensional plug flow simulation results. Even though large radial gradients were present in the tube reactor, the difference between the cross-sectional averaged profiles from $2 \mathrm{D}$ results and the one-dimensional plug flow results were negligible. 


\section{Chapter 5}

\section{Chemical Analysis of Fuel \\ Oxidation in Supersonic \\ Combustor}

\subsection{Turbulence and Combustion}

Hypersonic flight speeds impose strict engineering requirements on combustors. The high stagnation temperatures result in the dissociation of the upstream mixture of air which changes engine performance and introduces uncertainties in designing a hypersonic combustor. In addition, as the flight speed increases beyond certain Mach number, it becomes more efficient to maintain the flow at supersonic speed throughout the engine and combustor, because subsonic combustion at these condition will result in significant stagnation pressure loss [3].

Even more complications are introduced by the restriction on the combustion time of hypersonic combustors. The typical time available for proper fuel injection, fuel-air mixing, and combustion is on the order of $1 \mathrm{~ms}$ or less in hypersonic flow speeds. Such small time scales are orders of magnitude shorter in comparison to other standard 
combustion scenarios [97].

The different processes (i.e., flow, molecular transport, and chemical reaction) in supersonic combustion occur at time scales that differ by orders of magnitude (see Figure 5.1). Chemical reactions in general cover a wide time scale range from $10^{-10}$ s to more than 1 [98]. On the other hand, the physical time scales like molecular transport cover a much smaller range $\left(10^{-4} \mathrm{~s}\right.$ to $\left.10^{-2} \mathrm{~s}\right)$. An accurate modeling of supersonic reacting flow will require resolving the effects of all of these time scales in the simulation. However, a simplified model can be adapted for these problems. The fast chemical processes correspond to equilibrium conditions and the very slow chemical processes correspond to frozen conditions. In these cases chemical processes can be decoupled from the flow-field processes.

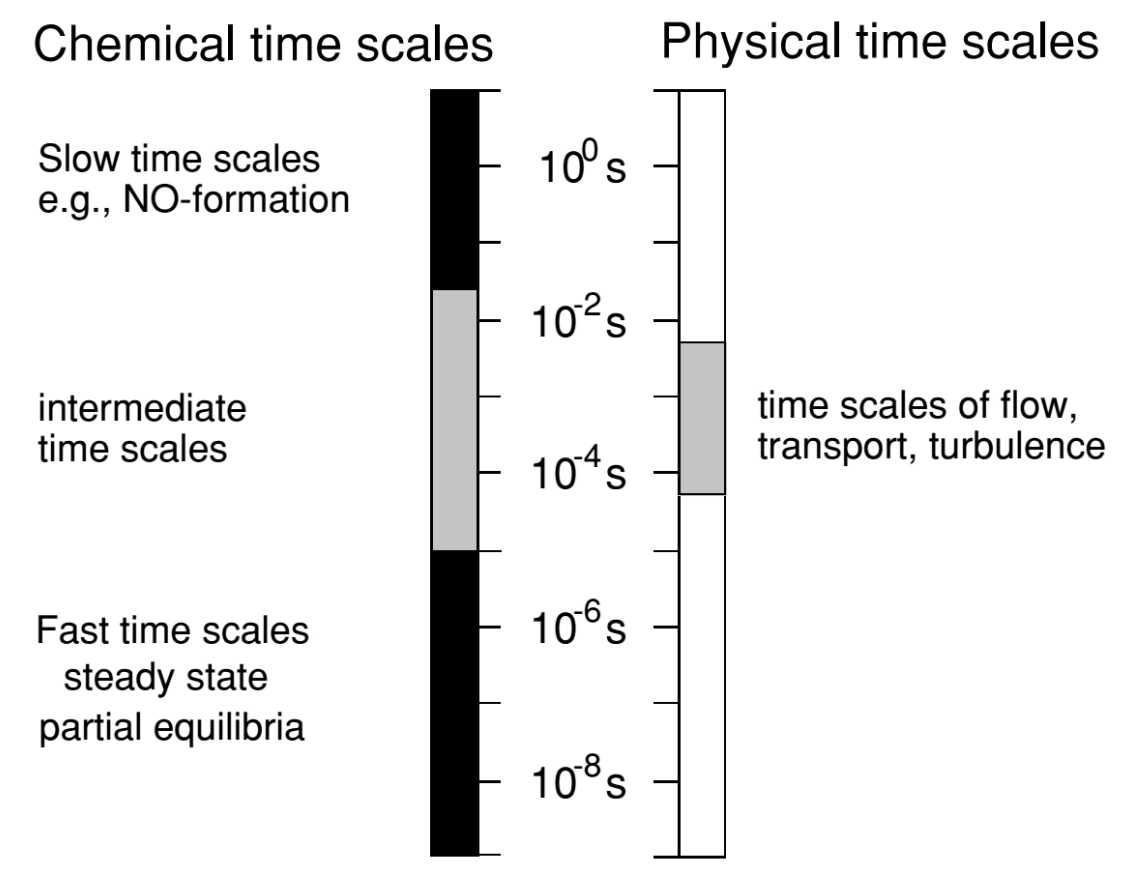

Figure 5.1: Time scales in chemically reacting flows [99].

Damkohler number is one of the most important nondimensional parameters for characterizing reacting flow regimes. Here, the Damkohler number corresponds to 
the ratio of the turbulent time scale to the chemical time scale:

$$
D a=\frac{\tau_{t}}{\tau_{c}}
$$

In the limit of high Damkohler number (Da $>>1)$, the chemical time scale is short compared to the turbulent time scale and this corresponds to a flame with thin reaction zone. The flame structure is not affected by turbulence and is described as a laminar flame element (flamelet regime) [100]. On the other hand, low Damkohler number $(\mathrm{Da}<<1)$ corresponds to slow chemical reaction with respect to flow speed. Reactants and products are rapidly mixed by turbulent structures before reaction. This limit is the perfectly stirred reactor regime. However, these situations rarely happen in supersonic combustion condition since the Damkohler number is around $1[2]$.

The University of Virginia Supersonic Combustion Facility (UVaSCF) is a smallscale experimental dual-mode scramjet combustor which is capable of simulating up to Mach 5 flight enthalpy (total temperature of $1200 \mathrm{~K}$ ) condition. It provides a clean test flow by electrical heaters that is free of any contaminates such as those produced by vitiation heaters $[101,102]$. The facility consists of an inlet nozzle, constant-area isolator, combustor with caivty flame-holder, and diverging extender section. The UVaSCF is investigating a cavity flame-holder due to its promise of providing both ideal combustion performance and low drag in supersonic combustion. The cavity provides a low-speed recirculation zone to increase the residence time of the fuel and air flow, for complete mixing and reacting process. It also provides a source of heat and radicals to ignite and stabilize combustion in the main flow (flame stabilization). The UVaSCF experiments use various diagnostic techniques that are capable of measuring static pressure, temperature, major species concentrations, three-component mean velocity and turbulent velocity. Figure 5.2(a) shows the schematic of the UVa dualmode combustor and figure 5.2(b) shows an image of the cavity flame-holder with the 
combustion of ethylene-air at Mach 2 condition.

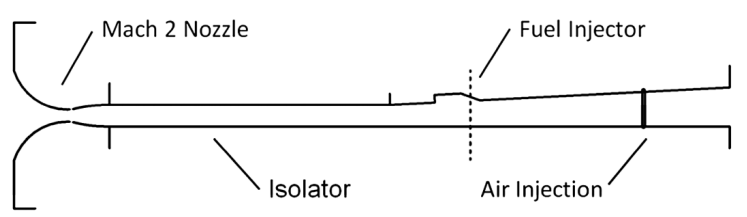

(a) Experimental configuration

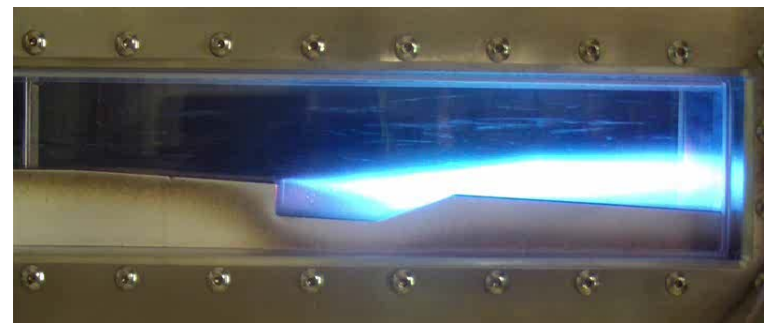

(b) Ethylene-air combustion at Mach 2

Figure 5.2: UVaSCF flowpath with cavity flame-holder [102].

In this work, the experimental conditions and parameters from the UVaSCF combustor are used to perform chemical reaction simulations with detailed chemistry models. In order to model the turbulence/chemistry interactions and fuel/air mixing phenomena with a simplified representation, perfectly stirred reactor (PSR) and partially stirred reactor (PaSR) models are used for the simulation of mixing-reacting flow within the cavity and the flame in the main flow, respectively. Based on the analysis performed under these realistic conditions a reduced chemical kinetic model for ethylene-air combustion is developed with great performance for a wide range of hypersonic operating conditions.

\subsection{Partially Stirred Reactor (PaSR) analysis}

\subsubsection{Introduction and Description of PaSR}

Real supersonic combustors are known to operate under intense turbulent mixing conditions. For such reacting turbulent flows, the partially-stirred reactor (PaSR) configuration has been used in the past to better understand the coupling between 
chemical reactions and the species mixing process in a computationally efficient manner [103-105].

In the PaSR reactor, the reactive flow field is assumed to be spatially homogeneous, hence the spatial fluid dynamics are not resolved. However, the reactive fluid components (i.e., reactants, intermediates, products, and temperature) are not perfectly mixed at the molecular level. The mixing process in the PaSR is characterized by the mixing frequency, which is modeled by the turbulence time scale. Because fluid dynamics inside the PaSR are not resolved, the mixing frequency will be prescribed as an input parameter. Therefore, in addition to the mean reactor residence time, the mixing time is another fluid mechanical time scale that controls the properties of the PaSR.

The composition and temperature in the PaSR are described by a probability density function (PDF), represented by $N$ number of reactive parcels or pockets of reactive gas. The reactor is assumed to be adiabatic and spatially homogeneous, thus the particle properties are only a function of time, which corresponds to a zerodimensional evolution in time. This does not imply they are perfectly mixed at the molecular level. The mass flow rate of particles entering and leaving the reactor are assumed to be equal $(\dot{m})$, hence the mass inside the reactor $(\mathrm{m})$ remains constant. The characteristic residence time $\left(\tau_{\text {res }}\right)$ specifies the mass flow rate entering the reactor by,

$$
\tau_{\text {res }}=\frac{m}{\dot{m}}
$$

To simulate turbulence effect in the reactor, a molecular level mixing process is provided by pairing two randomly selected particles for a specified time. The mixing intensity is modeled through the characteristic mixing time, $\tau_{m i x}$. This turbulent mixing time scale is proportional to the ratio of turbulent kinetic energy to turbulent 
dissipation rate:

$$
\tau_{\operatorname{mix}}=\frac{k}{\epsilon}
$$

There are two extreme limits in the PaSR model. When the mixing time is very small compared to the residence time and chemical time, the PaSR simulation approaches the perfectly-stirred reactor (PSR) where properties inside the reactor are homogeneously mixed at the molecular level. In this PSR limit, the residence time is the only controlling time scale. At the other limit, i.e. when mixing time scale is much longer than the residence time, the reactive gas pockets evolve in time as separate reactive mixtures with no mixing between them. As a result, the average of the PDF in the PaSR acts like a plug-flow reactor simulation [103, 104].

The PaSR can be used as a stand-alone model for studying turbulent combustion or any reactor systems where mass transport and species mixing is a rate-limiting factor. For example, PaSR can be used to simulate the sub-grid turbulent mixing and chemical reactions in a computational fluid dynamic (CFD) cell. The simplicity of this model allows to explore the effect of interaction between chemical reactions and fluid dynamics (turbulence) using detailed chemistry models with a significantly efficient computational cost compared to full reacting CFD simulation. In turbulent reactive flows, the coupling between chemical reactions and turbulence is very important. As mentioned before, the best parameter that characterizes the interaction between chemical reactions and fluid dynamics is the Damkohler number, which is defined as the ratio of characteristic flow time and the characteristic chemical reaction time. When the Damkohler number is of the order of unity, the interactions between the reaction and the fluid dynamics are strong and is considered rate-controlling condition. Otherwise the reactions can approach equilibrium condition(at very large $D a$ ) or frozen condition (at very small $D a$ ). The Damkohler number corresponding to the supersonic combustors are of the order of unity which makes the PaSR model 
a suitable representation for the turbulent mixing and chemical reaction interaction.

\subsubsection{Supersonic Combustion Reactor Configuration}

The University of Virginia's Supersonic Combustion Facility (SCF) is a dual-mode (ramjet/scramjet) combustor apparatus whose operation is designed to simulate flight conditions at Mach 5 enthalpy. It is vertically mounted and consists of an inlet nozzle (Mach 2 nozzle in the current case), a constant-area isolator, a combustor, and an extender, through which the exhaust gases are vented to the atmosphere. A schematic of the facility with the combustor dimensions is shown in Figure 5.3 and Figure 5.4, respectively.

The incoming air is compressed to a stagnation pressure of roughly $300 \mathrm{kPa}$ using an oil-free compressor, and a $300 \mathrm{~kW}$, 14-stage electrical resistance heater raises the stagnation temperature to $1200 \mathrm{~K}$ without introducing flow vitiates which is a significant advantage of this facility. The Mach 2 inlet nozzle, located upstream of the combustor, is responsible for bringing the incoming airflow up to the speeds at the isolator entrance that would normally be seen during low hypersonic flight, after the freestream air has been slowed and compressed in the inlet section of a hypersonic aircraft. The experimental facility is also fitted with a system of coolant lines, thermocouples, and pressure transducers for cooling of the supersonic combustor and data measurement. The facility is capable of operating continuously for long durations, limited only by the available fuel supply during combustion experiments [106]. 


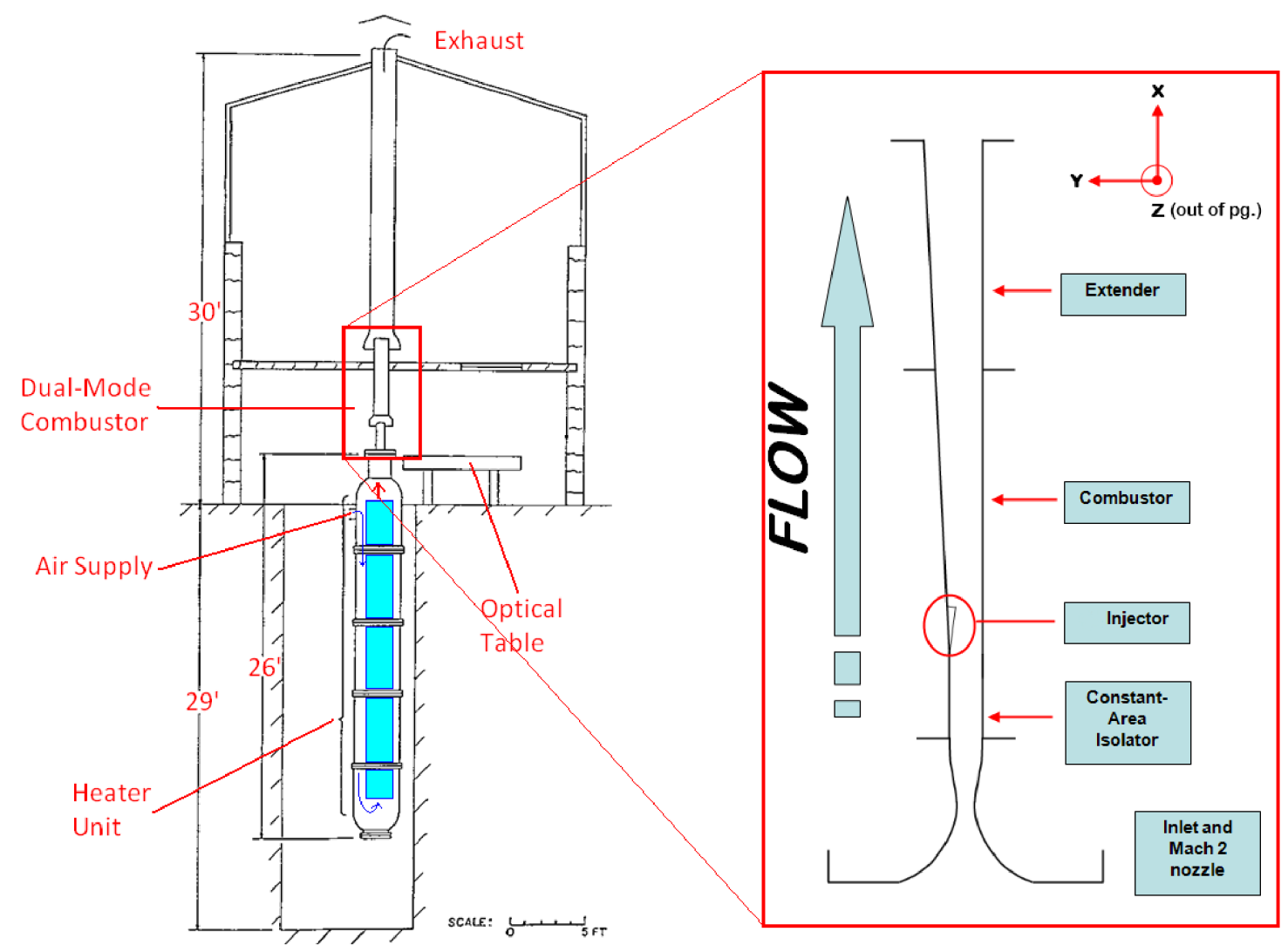

Figure 5.3: Schematic of the UVa supersonic combustion facility [106]. 


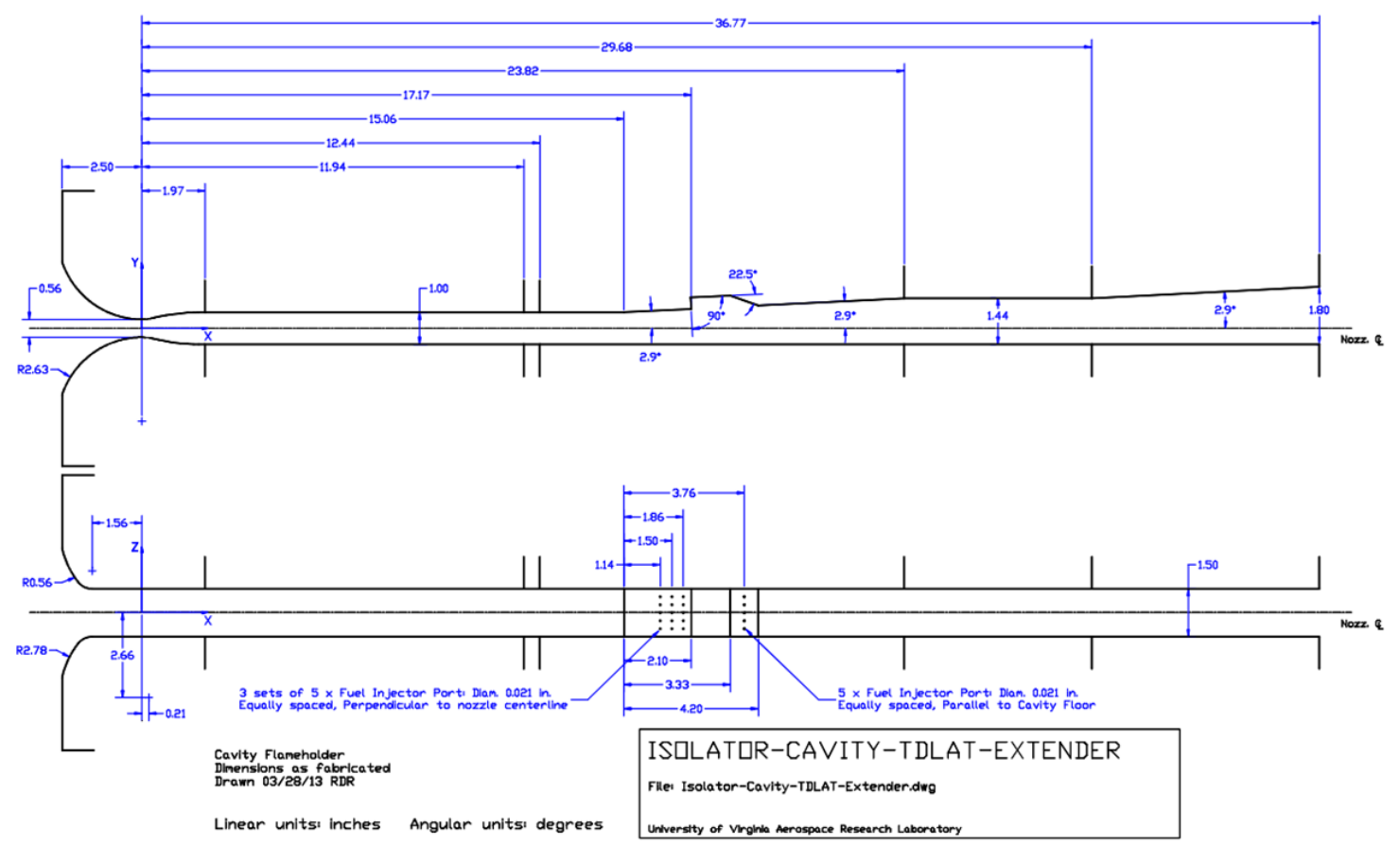

Figure 5.4: Dimensions of UVa supersonic combustion facility, reactor configuration E, with the Mach 2 nozzle, side view (top) and top view (bottom) [107].

Ethylene is used as fuel in this configuration of the combustor and is injected through the ports upstream of the combustor shown in Figure 5.4. A cavity is used to increase the residence time and improve flame holding at critical condition with low fuel induction time. A $2.9^{\circ}$ divergence on the cavity-side wall starts upstream of the cavity leading edge and is maintained through the combustor and extender sections of the flowpath, with a constant area section located between the combustor and extender providing a thermal throat when the flowpath is operating in the dualmode. At lower equivalence ratios, the facility operates in scramjet mode; as the equivalence ratio is increased, it transitions to dual-mode operation and then to ramjet operation as the precombustion shock train moves upstream from the combustor and eventually stabilizes in the isolator region. The details of the facility is described elsewhere [106-108]. 


\subsubsection{Reactor Flow Characteristic Time Scales}

The UVa supersonic combustion reactor flow conditions with the inflow stagnation values for the Mach 2 nozzle are shown in Table 5.1.

Table 5.1: Experimental conditions for the scramjet combustor.

\begin{tabular}{ll}
\hline \hline Parameter & Value \\
\hline Nozzle Mach number & 2 \\
Total pressure & $300 \mathrm{kPa}$ \\
Total temperature & $1200 \mathrm{~K}$ \\
Combustor inflow static pressure & $38 \mathrm{kPa}$ \\
Combustor inflow static temperature & $667 \mathrm{~K}$ \\
Combustor inflow velocity & $1035 \mathrm{~m} / \mathrm{s}$ \\
\hline \hline
\end{tabular}

As the flow-field operating condition transits to ramjet mode (specially for combustion with higher equivalence ratio), the combustor flow conditions downstream of the shock train are listed in Table 5.2.

Table 5.2: Flow conditions for the ramjet combustor.

\begin{tabular}{ll}
\hline \hline Parameter & Value \\
\hline Mach number & 0.577 \\
Combustor inflow static pressure & $173 \mathrm{kPa}$ \\
Combustor inflow static temperature & $1125 \mathrm{~K}$ \\
Combustor inflow velocity & $390 \mathrm{~m} / \mathrm{s}$ \\
\hline \hline
\end{tabular}

Based on the geometry of the combustor and the flow velocities at different operating conditions, the flow residence time at the main stream (core flow) of the combustor ranges roughly from $0.1 \mathrm{~ms}$ to $0.4 \mathrm{~ms}$. A recent hybrid LES/RANS 3D reacting flows simulation of the UVa supersonic combustor studied by Edwards and coworkers $[109,110]$, have calculated the residence time in the cavity. Figure 5.5 shows the residence time distributions computed from an ensemble of streamlines entering and leaving the cavity for the scramjet mode. It is shown there that the most probable residence time is $2.2 \mathrm{~ms}$, which is in good agreement with residence-time measurements obtained by monitoring the TDLAS temperature and water-column- 
density signal response during lean blowout condition [111]. This 2 ms residence time is used as the cavity residence time for the modeling purposes in this work.

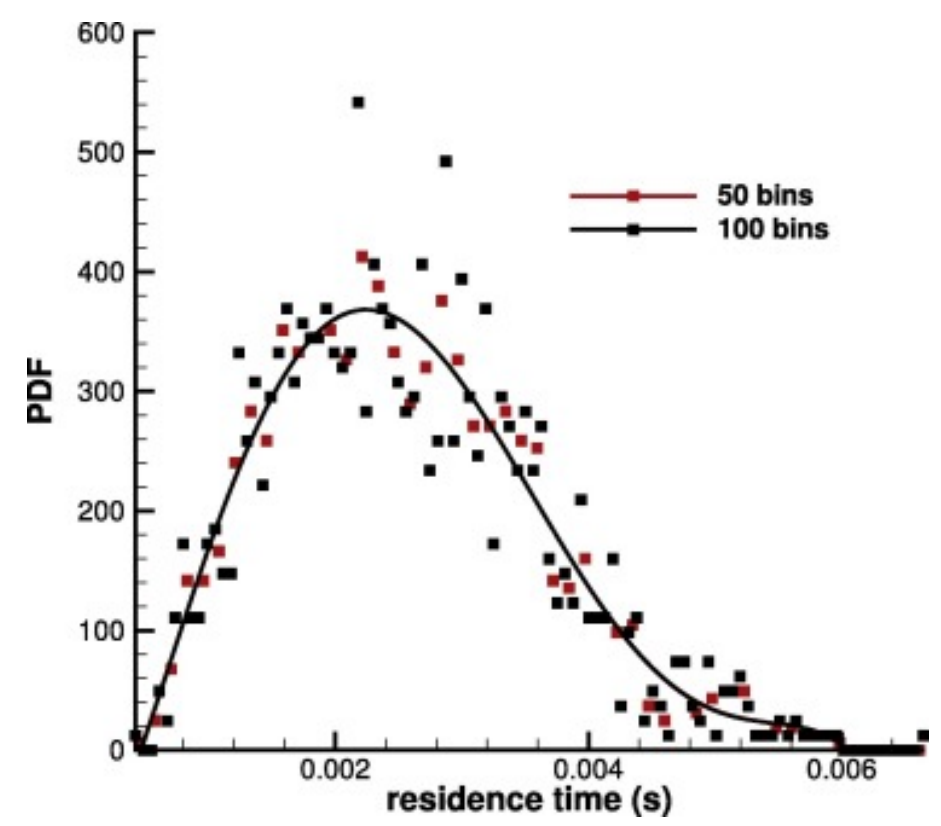

Figure 5.5: Cavity residence time distribution [109].

\subsubsection{PaSR Modeling Conditions}

The partially stirred reactor model is an adiabatic constant pressure reactor which requires these input parameters: 1) $\mathrm{N}$ number of particles in the reactor, 2) initial composition of the reactor, 3) residence time constant, 4) mixing time constant, 5) M inlet steams with given composition and temperature, 6) ratio of each inflow streams, 7) chemical kinetic model, 8) reactor operating condition (i.e, pressure, and initial temperature). In this work, ethylene-air mixture with various equivalence ratios are used for the analysis. As shown in the next section, two inflow streams are introduced in the PaSR. One is the fresh ethylene-air mixture from the unreacted main flow, the other stream is the already reacted flow from the cavity which is modeled by a perfectly stirred reactor. Initially (at time $=0$ ), the reactor consists of $\mathrm{N}$ statistical particles with fresh mixture (first inlet stream) composition assigned to all of the particle as initial value. 
At each time step a number of particles are chosen as inflow and outflow particle based on the residence time of the reactor:

$$
N_{\text {in }}=N_{\text {out }}=N \times \frac{\Delta t}{\tau_{\text {res }}}
$$

Based on the ratio of the two inlet streams, a percentage of these inflow particles are assigned with the first stream temperature and composition and the rest are assigned with the properties of the second inlet stream. In order to assure a continuous flow of particles of both streams during each time step of the stochastic simulation, we used N=4000 particles in the reactor. Each particle is considered as an independent closed reactor. The properties of each particle changes due to two procedures, the molecular mixing process and the chemical reaction. At each time step the composition of the particles evolve as a result of the chemical kinetic reactions and then interact with other particles to exchange properties. The mixing rate between two particles a and $b$ are defined by the mixing time as:

$$
\frac{\Delta Y^{a}}{\Delta t}=-\frac{Y^{a}-Y^{b}}{\tau_{m i x}}
$$

The two particles that exchange properties as the mixing process, are randomly selected and matched at each time step. This random shuffling between the paired particles help the stochastic procedure to be uniformly distributed.

Figure 5.6 shows the schematic of the mixing process of the particles in the PaSR model at a particular time. All the particles provide a probability distribution as a function of time for the thermochemical properties such as temperature and species mole fractions. 

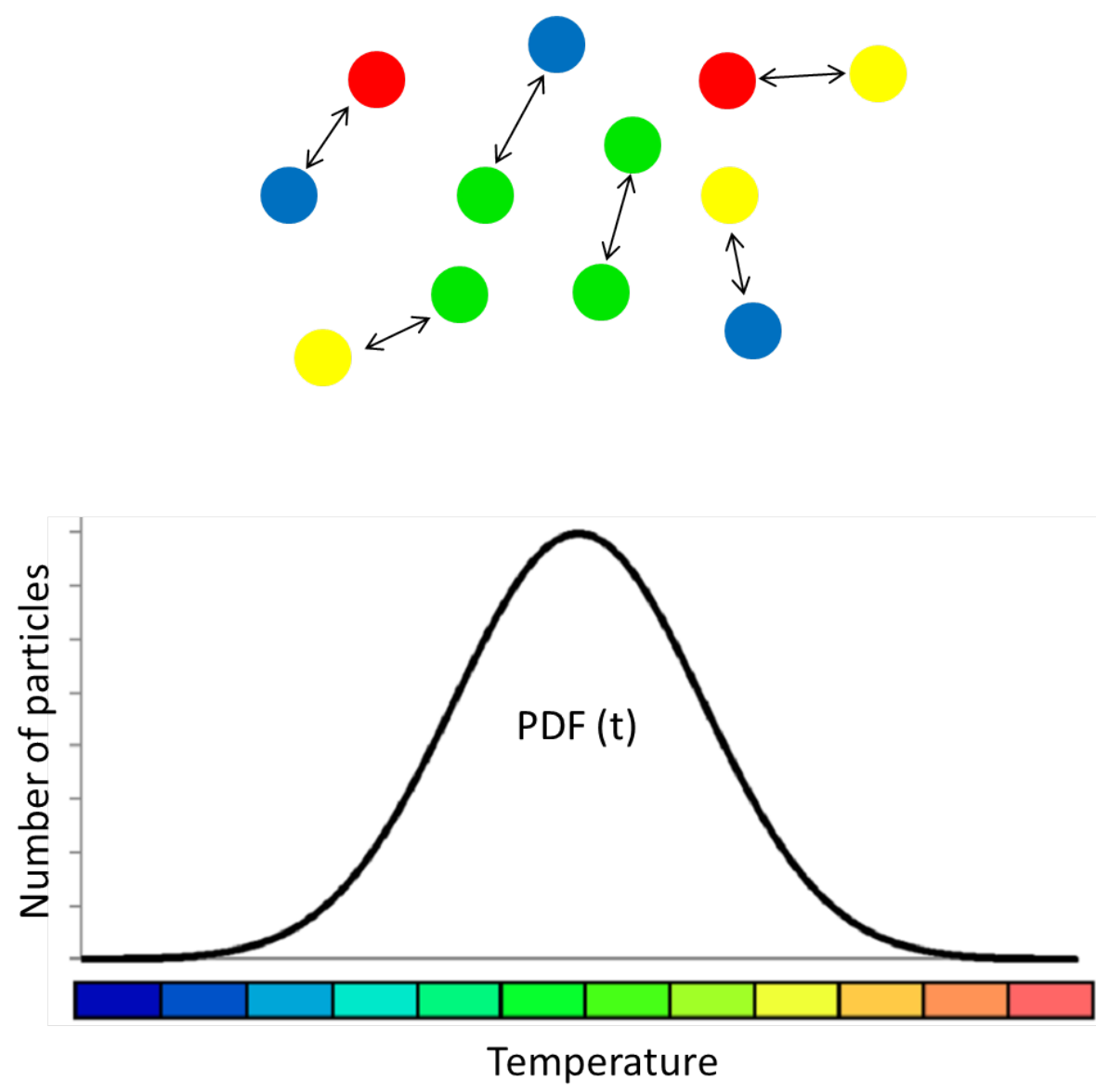

Figure 5.6: Schematic of the stochastic mixing of the PaSR model, represented by N particles with the probability distribution of temperature and mass fraction changing as a function time.

To find the mean composition and temperature of the PaSR reactor, the average of all the available particles inside the reactor are calculated at each time step. Since the mass is conserved in the PaSR, the outflow stream is the same number of particles as the total inflow, except they are randomly selected from the particles inside the reactor. As previously stated, based on the experimental flow configuration and turbulent intensity, the relevant residence time for the PaSR simulation range from $100 \mu$ s to $400 \mu$ s and the mixing times are between $10 \mu$ s to $40 \mu$ s for the current experimental conditions.

Figure 5.7 shows the schematic of PaSR model for the hypersonic combustor. Upstream flow is the premixed unreacted ethylene flow considered as the first inlet 
stream in the PaSR model. The recirculation region in the cavity provides the reacted mixture (radical pool) as the second inlet stream into the PaSR model. The composition of the cavity flow will be calculated by perfectly stirred reactor (PSR) model. The PSR residence time is determined by the cavity flow residence time. Inside the PaSR reactor there is a mixture of particles with different compositions and temperatures which are determined by the chemical reactions, mixing process and most importantly each particle residence time. The outflow stream is by removing a random selection of particles from inside the reactor and replacing them with the inlet stream particles.

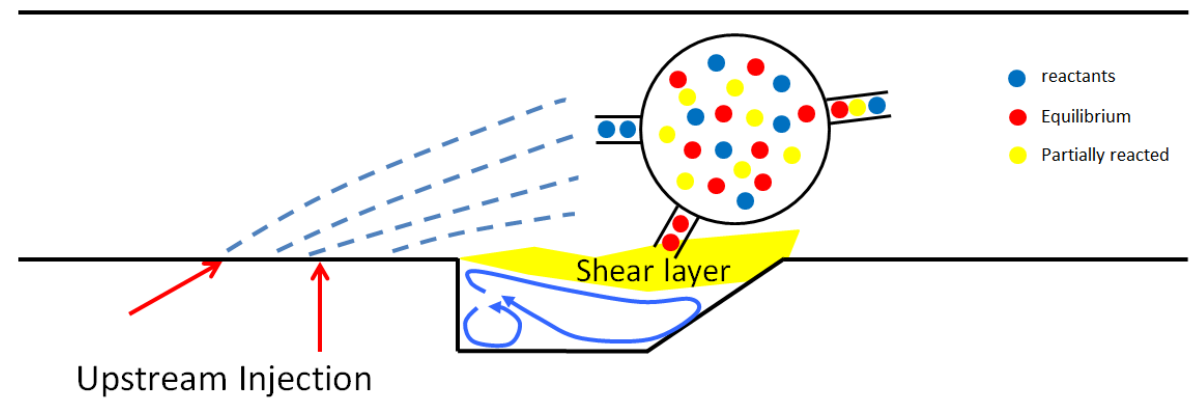

Figure 5.7: Injector and combustion schematic with PaSR modeling.

The simplicity of this Monte Carlo random simulation of PaSR enables us to perform reaction simulation with turbulent mixing interactions using detailed chemistry mechanisms without significant computation cost. To reduce even more the computational cost of the chemical reaction modeling, a storage retrieval method of in-situ adaptive tabulation (ISAT) [105] has been implemented for the PaSR simulation. This implementation enabled the investigator to perform extensive sensitivity analysis for PaSR simulations for a range of conditions in a computationally efficient manner. These computations where performed on the UVa high-performance computing cluster using more than 10000 CPU-hours. The results where used to develop a robust reduced model good for a wide range of conditions. 


\subsubsection{PaSR Results}

Figure 5.8 shows the temperature profile of the partially stirred reactor. Three different reactor initial temperatures of 1000, 1125, and $1200 \mathrm{~K}$ are explored. The residence time is $200 \mu \mathrm{s}$ and the mixing time is $20 \mu \mathrm{s}$. The ratio between the two streams are $98 \%$ main flow (fresh mixture) and $2 \%$ cavity flow (PSR mixture) with ethylene-air equivalence ratio of 0.45 . As the reactor initial temperature increases two properties of the reactor change significantly. The steady state temperature, the temperature at enough long simulation time, increases with approximately the same amount. Also the mixture induction time is reduced significantly. The red solid line in this figure corresponds to the ramjet mode limit condition in the supersonic combustor with Mach 2 nozzle. The induction time at this condition is about $0.25 \mathrm{~ms}$.

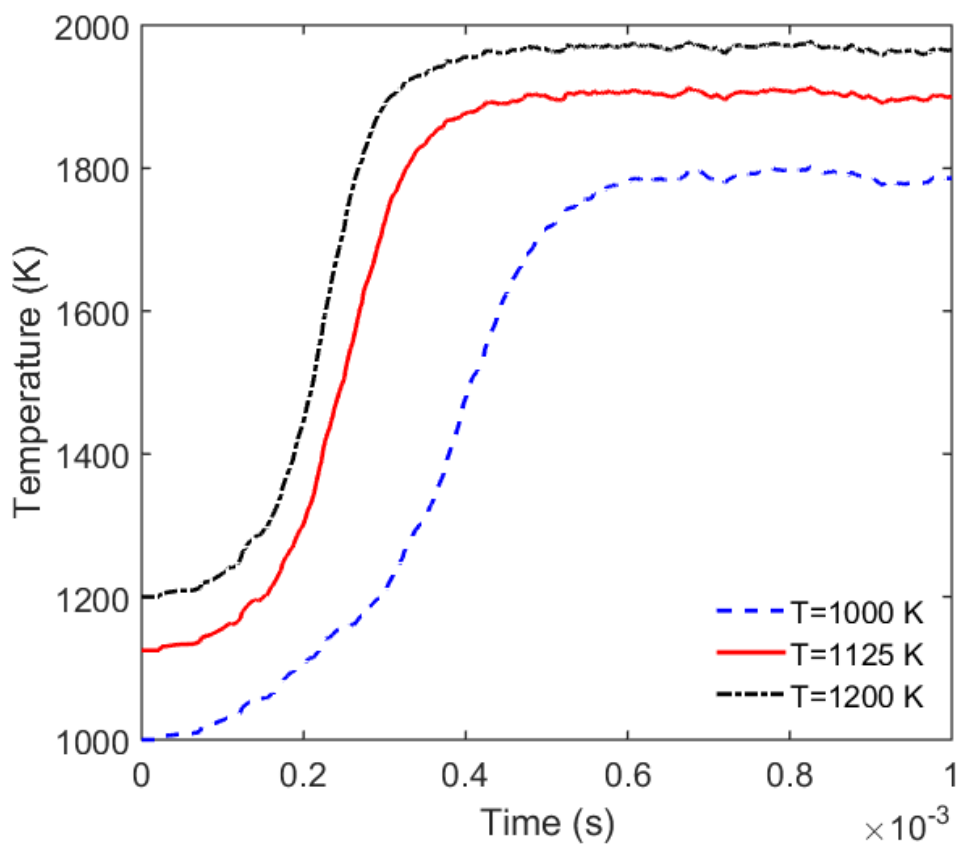

Figure 5.8: PaSR temperature profile for different reactor initial temperatures.

Figure 5.9 shows the temperature profiles of the partially stirred reactor with three different residence times of 100,200 , and $400 \mu \mathrm{s}$. The reactor initial temperature is $1125 \mathrm{~K}$ and the mixing time is $20 \mu \mathrm{s}$. The ratio between the two streams are $98 \%$ main 
flow (fresh mixture) and $2 \%$ cavity flow (PSR mixture) with ethylene-air equivalence ratio of 0.45 . By increasing the residence time, the reactor reaches higher temperature at steady state condition. The reason is that the mixture inside the reactor has longer available time for chemical reaction. But this parameter has a negligible effect on the induction time. The induction time for all the three residence time is about the same $0.25 \mathrm{~ms}$.

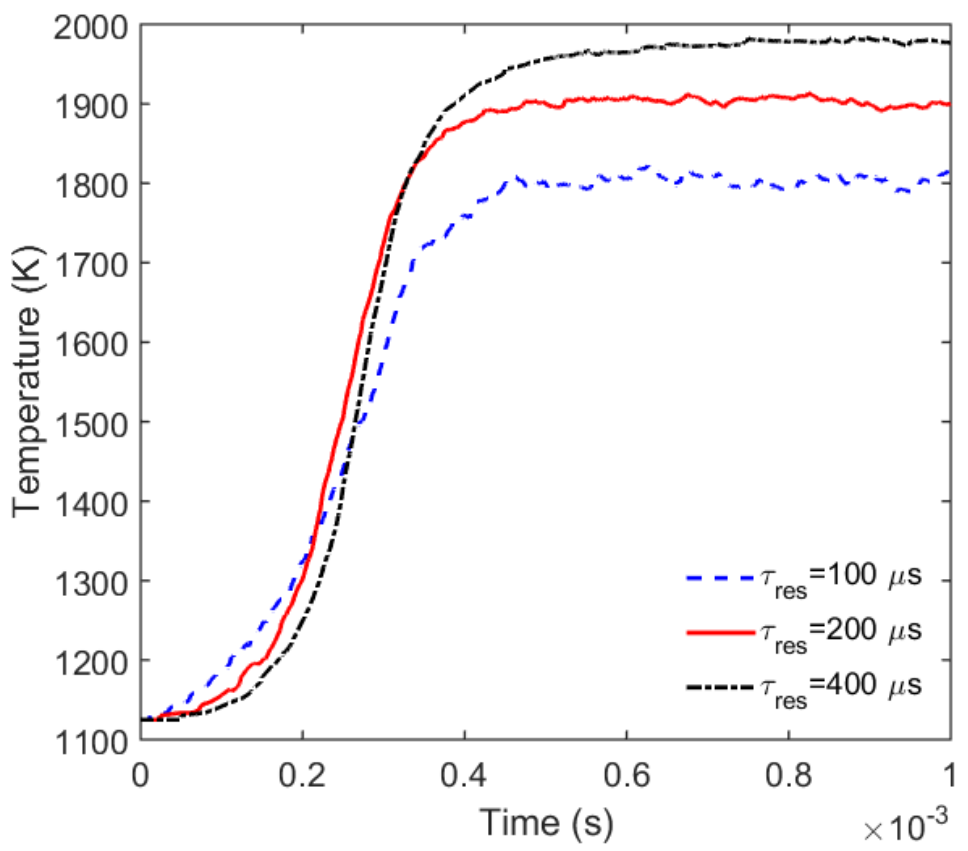

Figure 5.9: PaSR temperature profile for different reactor residence times.

Figure 5.10 shows the temperature profiles of the partially stirred reactor with three different mixing times of 10,20 , and $40 \mu \mathrm{s}$. The reactor initial temperatures is $1125 \mathrm{~K}$ and the residence time is $200 \mu$ s. The ratio between the two streams are $98 \%$ main flow (fresh mixture) and $2 \%$ cavity flow (PSR mixture) with ethylene-air equivalence ratio of 0.45 .

Changing mixing time has minimal effect on both steady state temperature and induction time. Longer mixing time means lower turbulent intensity and slower mixing. Therefore the induction time is longer at higher mixing times but not significantly. Also the temperature at steady state region is few degrees lower. For most of the 
analysis performed in this work, $20 \mu$ s was used as the default mixing time scale.

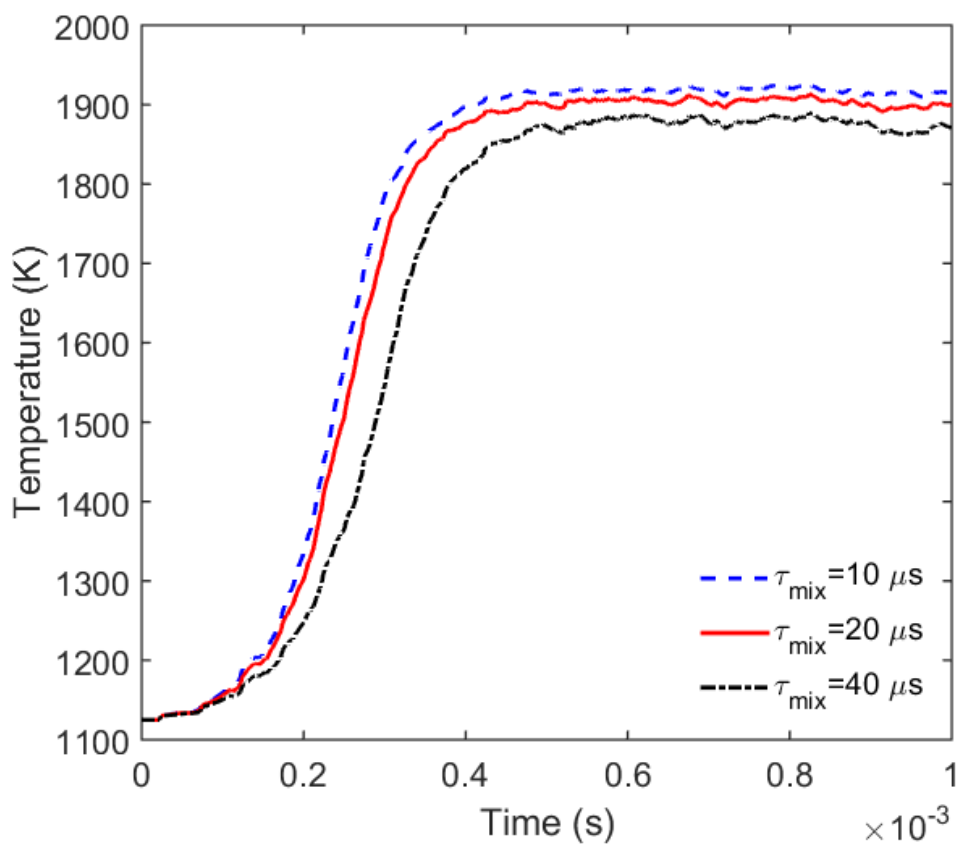

Figure 5.10: PaSR temperature profile for different reactor mixing times.

Figure 5.11 shows the temperature profiles of the partially stirred reactor as a function of the ratio between the two inlet streams. Three different flow ratios of the cavity flow to main flow are explored, $1 \%, 2 \%$, and 5\%. The reactor initial temperatures is $1125 \mathrm{~K}$ and the residence time and mixing time are $200 \mu \mathrm{s}$ and $20 \mu \mathrm{s}$, respectively. This figure shows that by changing the cavity flow percentage from $1 \%$ to $2 \%$, the induction time is reduced considerably. However, after that the mixture is saturated by radicals that any further increase of the cavity flow does not effect the reaction conditions. Also the steady state temperature is not effected by the amount of the cavity flow in the reactor. 


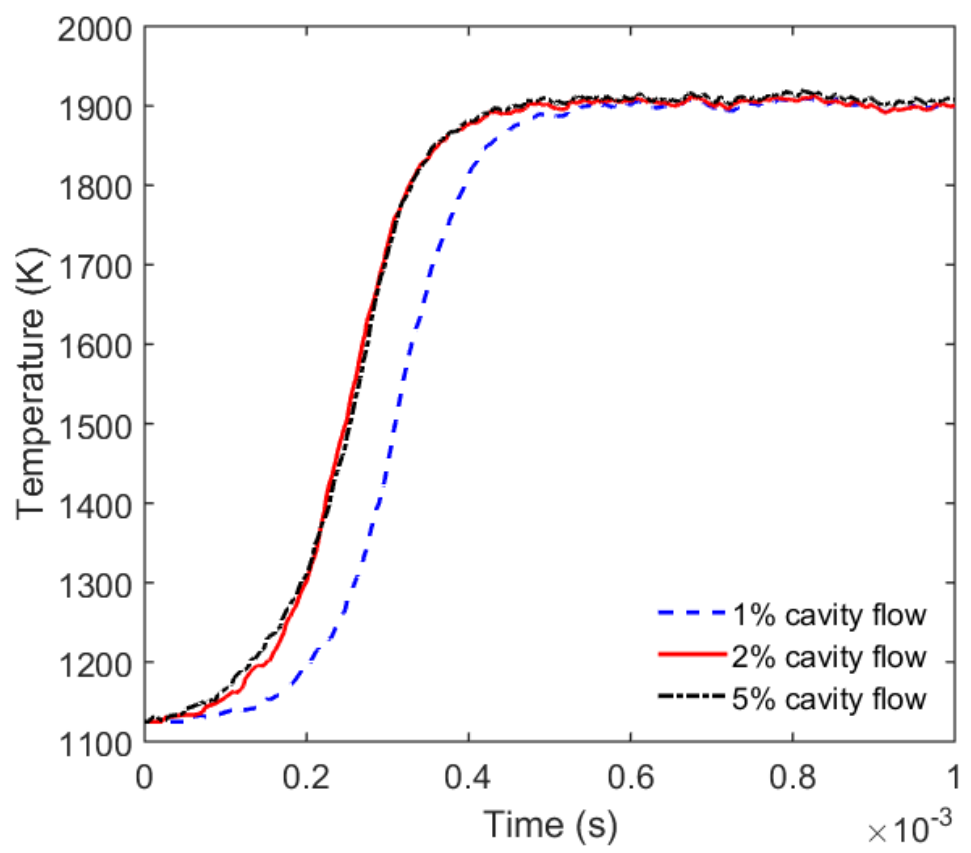

Figure 5.11: Temperature profile of PaSR for various ratios of the cavity flow to the main flow.

\subsection{Ignition Delay Time Analysis}

In high-speed reacting flows with relatively long mixing time scales compared to the advection time scales, homogeneous ignition chemistry can describe the induction length or the location where intense reactions occur. For this reason, accurate prediction of homogeneous ignition phenomenon under various initial conditions (i.e. temperature, pressure, and equivalence ratios) and fuel-air mixtures needs considerable attention. In particular, considerable effort has been devoted to measure ignition delay times in shock tubes as well as temporal evolution of temperature and species, providing a valuable database for chemical kinetic model optimization. For example, Hong et al. [38] have proposed a new hydrogen kinetic model based on the ignition delay and speciation information. Burke et al. [39] have also used the same ignition database together with their high-pressure flow reactor database to develop a slightly modified hydrogen kinetic model. 
Predicting the induction time in the hypersonic combustor is critical because of the flame holding issue and preventing flame extinguishing of the combustor in the scramjet mode. For example in the current setup of UVa supersonic combustor, at the ramjet mode, the equivalence ratio is limited by the thermal choking is 0.45 . At this condition the ignition delay time of the ethylene-air mixture is about $1.4 \mathrm{~ms}$. But at scramjet mode the conditions are different. The equivalence ratio limited by the thermal choking is 0.25 and the flow temperature is about $700 \mathrm{~K}$. The induction time for these conditions is significantly larger which means the combustor cannot flame hold without the cavity. Figure 5.12 shows ethylene-air mixture ignition delay time as a function of initial temperature for three different equivalence ratios. As the overall equivalence ratio increases, the ignition time becomes shorter.

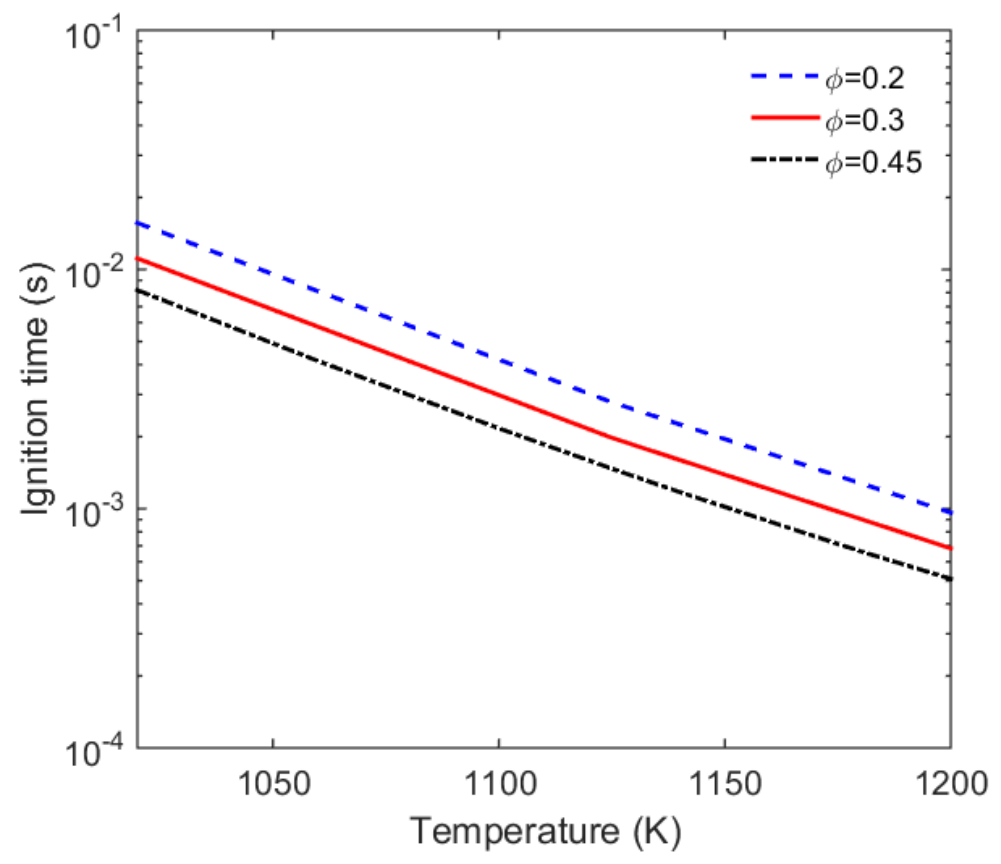

Figure 5.12: Ignition delay time calculation for ramjet mode combustor at different equivalence ratios of ethylene-air combustion.

Figure 5.13 depicts the schematic of the UVa supersonic combustor when the main flow is considered completely premixed and the cavity is modeled by a perfectly stirred reactor. The residence time of the flow in the cavity is adopted as the PSR residence 
time for reaction calculation. Then the flow from both main flow and cavity are mixed in a closed homogeneous reactor with different split ratios. Ignition delay time calculation is performed based on the closed homogeneous reactor conditions after the two flow complete mixing. Figure 5.14 shows the ignition time calculations for selected cavity to main flow ratios between $0 \%$ to $5 \%$. As the ratio of the cavity flow to the main flow increases in the mixture, the ignition time decreases significantly. For the condition of interest (ramjet mode) with initial temperature $1125 \mathrm{~K}$, the ignition time is $1.5 \mathrm{~ms}$ for pure main flow mixture. Whereas the ignition delay time reaches down to $0.9,0.6$, and $0.44 \mathrm{~ms}$ when the main flow is mixed with $0.1 \%, 1 \%$, and $5 \%$ cavity flow (PSR mixture composition), respectively.

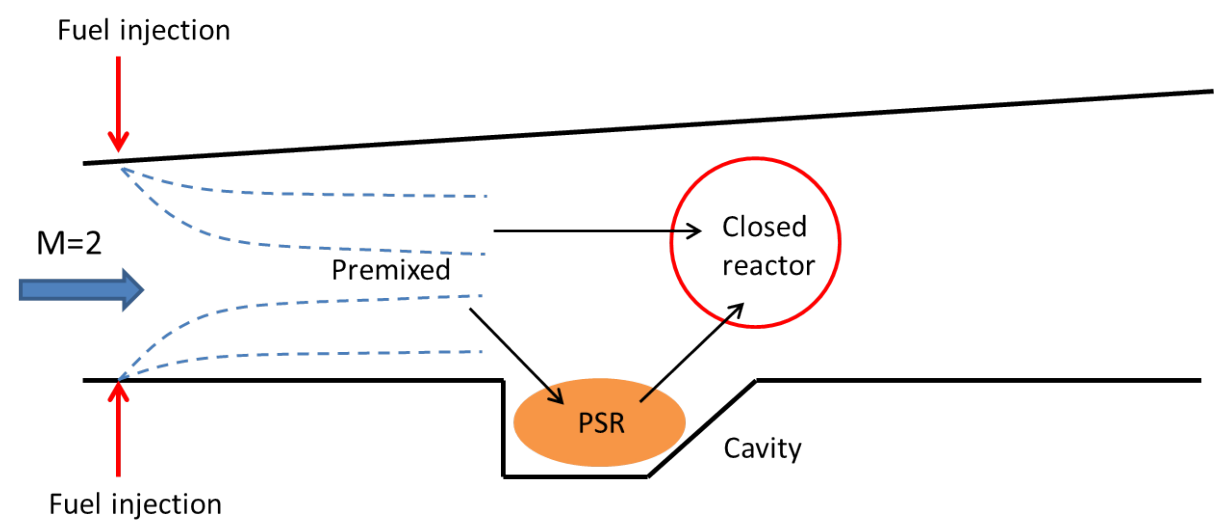

Figure 5.13: Schematic of the mixing cavity flow with the main flow and ignition in a closed homogeneous reactor. 


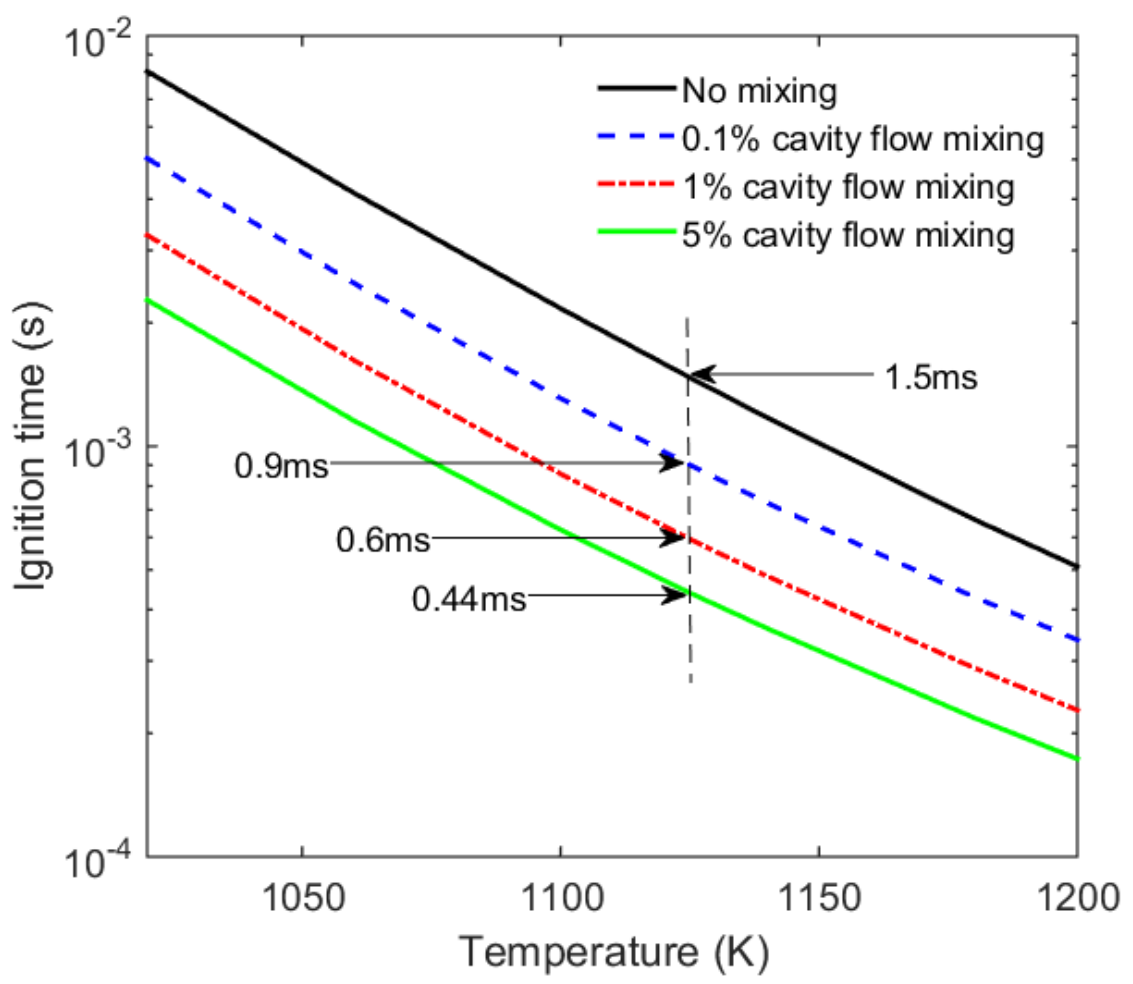

Figure 5.14: Ignition delay time as a function of inflow temperature for the ramjet mode ethylene-air combustion for different mixing percentages of cavity flow to the main flow.

Figure 5.15 depicts the schematic of the UVa supersonic combustor when the premixed main flow and the perfectly stirred reactor flow from the cavity are mixed as two separate inflow streams in a partially stirred reactor model. In this configuration the turbulent mixing-reacting interactions are captured and resolved in the PaSR model. To see the difference between the PaSR model and the previous closed reactor model, the ignition time is calculated for a range of temperatures and compared with the results from closed homogeneous reactor.

Figure 5.16 shows the ignition delay time results as a function of inflow temperatures from $1000 \mathrm{~K}$ to $1200 \mathrm{~K}$. The main flow is at default ramjet mode condition (1.73 atm). The PSR residence time is $2 \mathrm{~ms}$ which is same as the cavity flow residence time. These two separate flows are input streams in the PaSR reactor with time constants corresponding to the supersonic combustor flow time scales $(200 \mu$ s residence 
time and $20 \mu$ s mixing time). The ratio of the two inflow streams are another input parameter for the PaSR simulation.

As previously shown in Figure 5.11, the cavity flow ratio does not effect the PaSR prediction significantly. Therefore only the default $2 \%$ cavity flow to main flow ratio is shown in this figure. The fuel induction time is improved significantly in the PaSR model compared to the closed reactor, specially at lower temperatures where reaction is slower (see Figure 5.16). The fuel induction time is about $0.25 \mathrm{~ms}$ at flow conditions of $1125 \mathrm{~K}$ and $1.73 \mathrm{~atm}$. This low ignition time enhances the operability limits of the combustor at hypersonic speeds where the fluid residence time in the combustor is of the order of a few milliseconds. Here, the PaSR model has shown the improvement effect of turbulent mixing-reacting interactions in the flame stabilization for the combustor at limiting conditions.

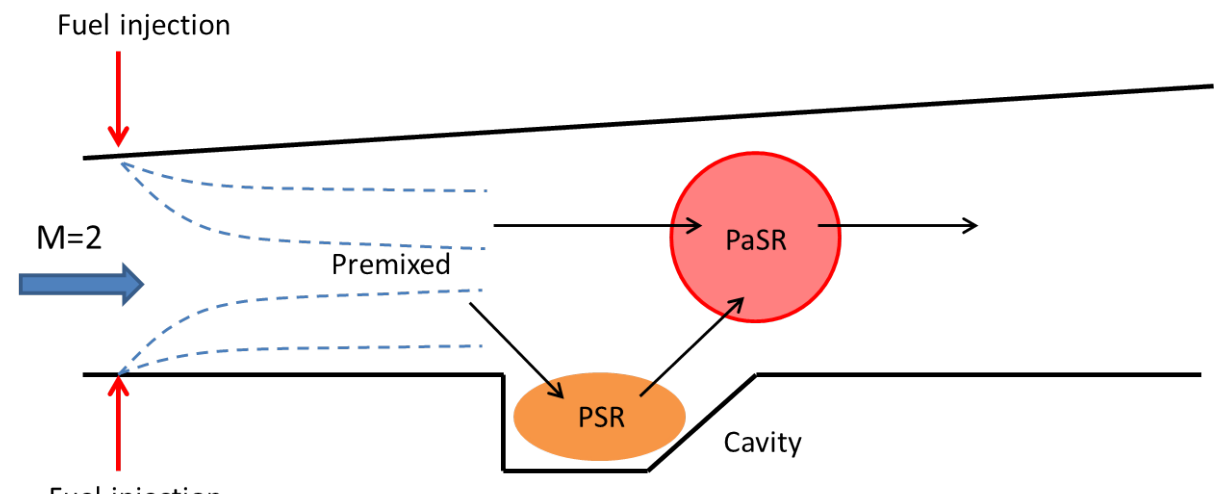

Figure 5.15: Schematic of the mixing cavity flow with the main flow in a partially stirred reactor. The cavity flow is modeled by a perfectly stirred reactor. 


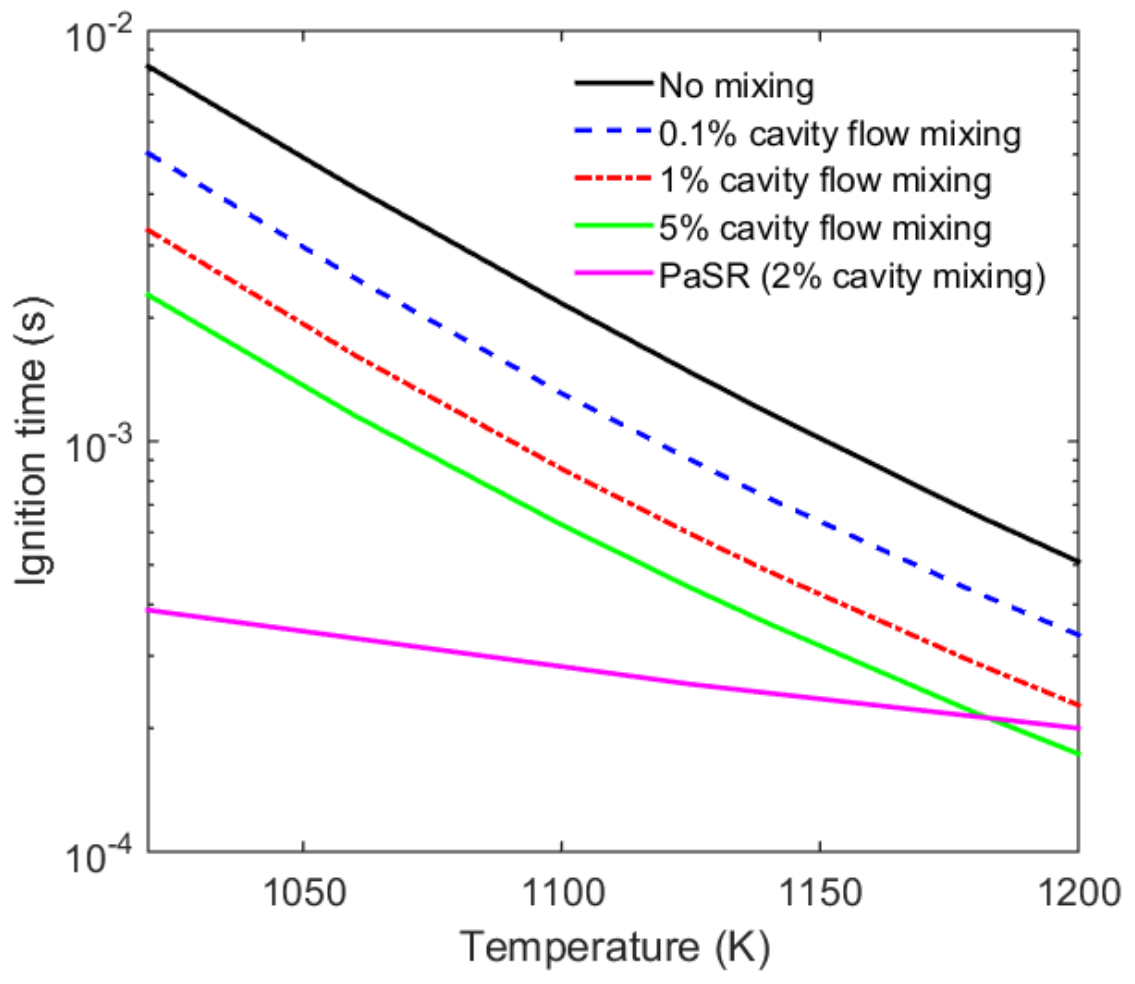

Figure 5.16: Comparison of ignition delay time between PaSR model and closed homogeneous reactor model for the ramjet mode ethylene-air combustion. The PaSR simulation consist of mixing $2 \%$ cavity flow (PSR mixture) with $98 \%$ unreacted main flow.

\subsection{Mechanism Reduction Based on PaSR}

\subsubsection{Ethylene Combustion Reduced Model}

To perform large scale multi-dimensional reacting flow simulations, applying the full detailed chemistry is prohibitively expensive. Even with the current computational resources, three-dimensional combustion simulation (i.e. DNS, LES, and RANS) with detailed chemistry is not practical. As a result, there is a substantial interest in the community to develop reduced chemical reaction models. However, most of these reduced mechanisms are suitable for certain mixture types and specific ranges of operating conditions. 
For example recent LES/RANS supersonic reacting flow simulation by Edwards and coworkers $[109,110]$ have used a reduced ethylene oxidation chemistry model recently developed by Tianfeng Lu and coworkers [112]. This reduced model is based on the University of Southern California detailed mechanism (USCMechII [70]). First, a skeletal mechanism with 32 species and 206 reactions was obtained by eliminating unimportant species and reactions using directed relation graph (DRG) [92] with sensitivity analysis. To reduce the number of transported variables and further reduce computational cost in 3D simulations, a quasi steady-state approximations (QSSA) was applied to 10 radicals $(\mathrm{CH}, \mathrm{CH} 2, \mathrm{CH} 2 *$, $\mathrm{HCO}, \mathrm{CH} 3 \mathrm{O}, \mathrm{H} 2 \mathrm{CC}, \mathrm{C} 2 \mathrm{H} 3, \mathrm{C} 2 \mathrm{H} 5$, $\mathrm{CH} 2 \mathrm{CHO}, \mathrm{nC} 3 \mathrm{H} 7)$. Therefore, a 22 species reduced mechanism with algebraic equations for the QSS species was obtained. It is shown that this reduced model performs well for a wide range of conditions.

In the current study, unlike the preceding example reduced model, a skeletal reduced reaction model is developed based on Principal Component Analysis with Sensitivity (PCAS) [93] in this work. The detailed reaction model JetSurF 2.0 [69] consisting of 348 species and 2163 reactions is used as starting mechanism which is extensively validated with various experimental measurements. The goal is to develop a much simplified reduced mechanism for ethylene-air reaction that can predict combustion phenomena such as ignition and flame propagation as accurate as the detailed model for the conditions of interest.

A new approach has been adopted in this work. In addition to the sensitivities of ignition calculation, sensitivities of partially stirred reactor model has also been included in the analysis. This way the effect of turbulent mixing-reacting interaction can be included to the analysis to develop a more robust reduced model for turbulent simulations. It has been shown in a recent paper by Rahimi et al. [113], that chemical kinetic mechanism reduction for ethylene oxidation using the PaSR model has significantly improved the performance of the skeletal model accuracy compared 
to skeletal models extracted from ignition delay time sensitivities even at very small number of species and reactions. Figure 5.17 shows the comparison of the normalized error of the reduced model predictions as a function of the number of species in the models for ethylene-air combustion at atmospheric pressure. As shown in this figure, the skeletal models developed based on the PaSR model sensitivities have smaller error compared to the models developed based on ignition time sensitivities.

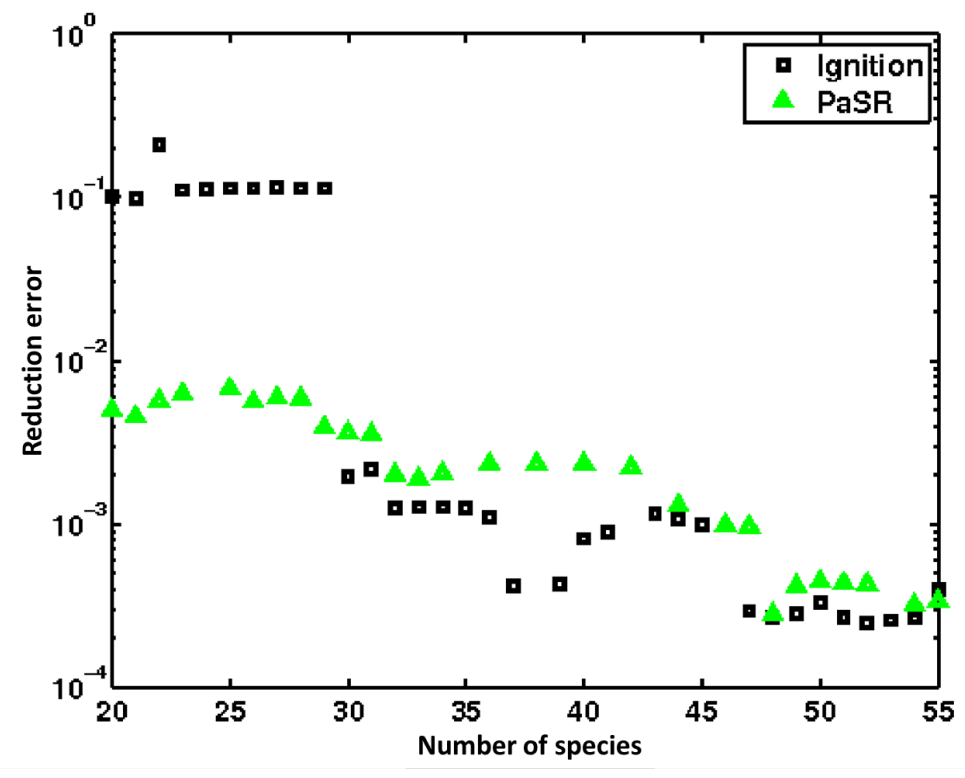

Figure 5.17: Model reduction error for a stoichiometric mixture of ethylene-air combustion.

Based on the various conditions explored, a reduced mechanism with 26 species and 132 reaction is developed which is given in detail in Appendix A. In the next following section, the model validation and accuracy of the prediction of this model is compared with other available mechanisms.

\subsubsection{Reduced Model Validation and Performance}

To provide the model validation of the 26 species reduced model, the ignition delay time predictions from this model is compared against other selected models for a wide range of conditions. First, the predictions of this model is compared with the 
detailed model JetSurF2.0 [69], the reduced model (2012) by Lu and coworkers [112], and the KAUST 2013 model [114]. Figure 5.18 shows the ethylene-air ignition time prediction of the 26 species model compared with the other three models for a range of temperatures from $900 \mathrm{~K}$ to $1700 \mathrm{~K}$. The mixture composition has stoichiometric ratio and at atmospheric pressure. As shown Figure 5.18, the prediction of the 26 species model developed in this work is in great agreement with the full detailed model. On the other hand, the two other models (Lu 2012 [112], KAUST 2013 [114]) have prediction discrepancies of more than factor of 2 . The ignition delay time prediction is specially important in critical operating conditions where the flame holding and stability is dependent on the flow residence time and the fuel induction time.

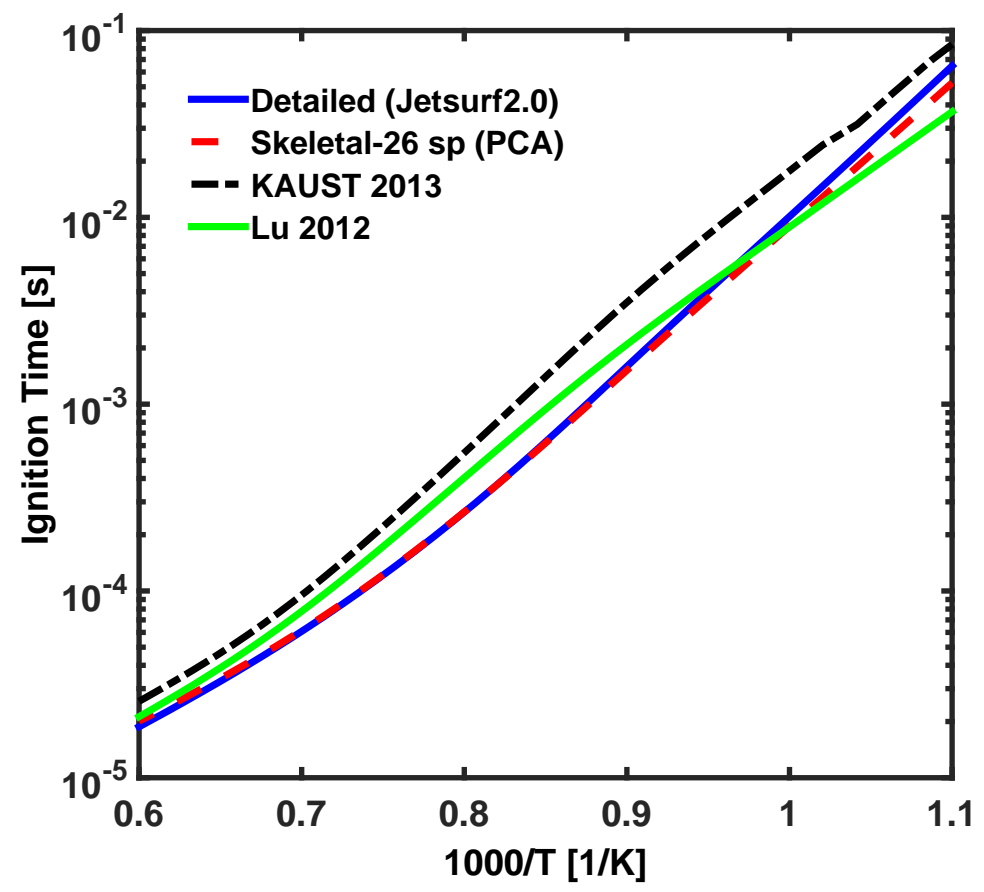

Figure 5.18: Ignition delay time as a function of initial temperature for ethylene-air stoichiometric mixture under atmospheric pressure.

To explore more extreme conditions, the ignition time is calculated for three equivalence ratios $(0.5,1$, and 2$)$ of ethylene-air mixtures and at two operating pressure conditions (1 atm and 10 atm). Figure 5.19 shows the comparison of the model pre- 
dictions of the 26 species model with the detailed model for the four combination of conditions. The mixture initial temperature ranges from $900 \mathrm{~K}$ to $1700 \mathrm{~K}$. It is evident that the reduced model can predict the ethylene-air combustion accurately at various operating conditions. This model has recently been used in LES/RANS simulation of the UVa supersonic combustor by Edwards and coworkers at NCSU and has improved the computation performance as well as reaction predictions.

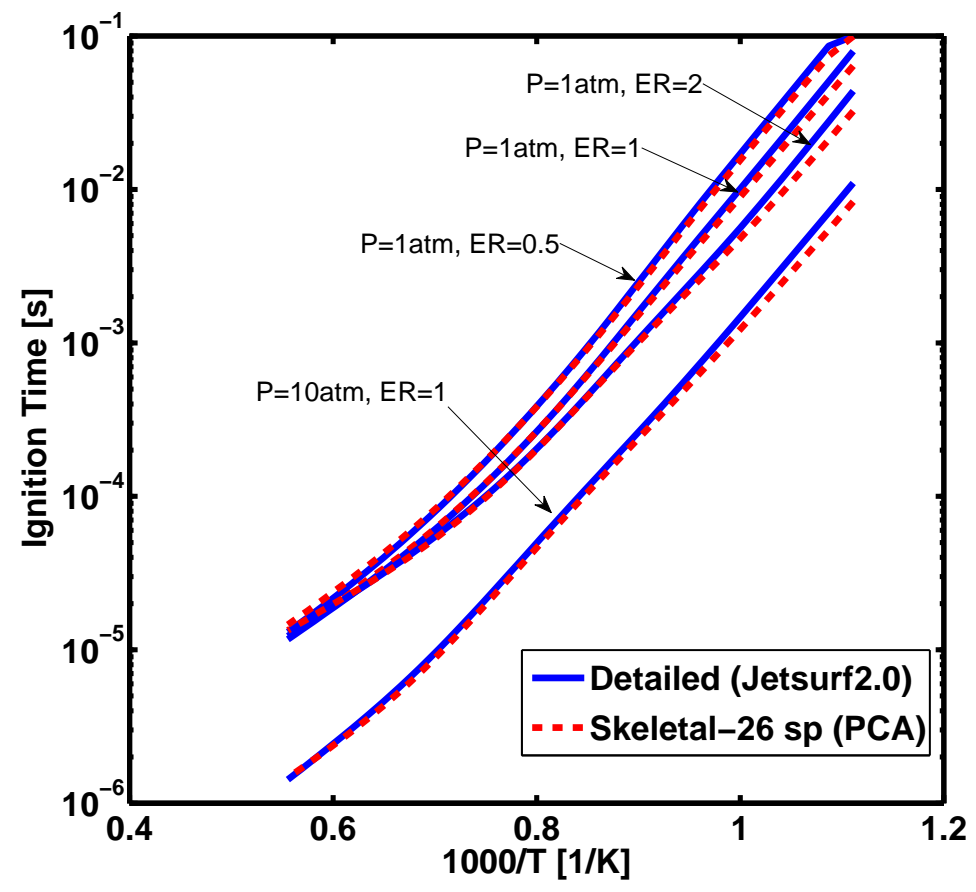

Figure 5.19: Comparison of ethylene ignition delay time of detailed and reduced model as a function of initial temperature for different reacting conditions. Pressure $1 \mathrm{~atm}$ and $10 \mathrm{~atm}$, and equivalence ratio of $0.5,1$, and 2 are shown.

\subsection{Summary}

A new approach to model the fuel oxidation in the dual-mode supersonic combustor was investigated. The cavity flame holder and the turbulent mixing and reaction interaction in the combustor were simulated by a computationally efficient implementation of partially stirred reactor model in connection with perfectly stirred reactor. 
The relevant time scales for hypersonic combustors were used for the simulations. The ignition delay time predictions from this analysis were much closer to the observed experimental flame holding results. This simplified method was recently used to design a new insert cavity flame holder for more improved combustion performance in the supersonic combustor. A reduced reaction mechanism for ethylene combustion was also developed. The predictions from this reduced model were validated for a range of applicable hypersonic conditions. 


\section{Chapter 6}

\section{Thermal Choking in Hypersonic Combustor}

In principle, the difference between a ramjet and a scramjet engine is the presence of a physical throat in the nozzle for ramjet with subsonic combustion, whereas the scramjet with supersonic combustion requires an area increase. A hypersonic propulsion system that can operate from Mach 3 to 10 and above, requires an integrated system that can perform both subsonic and supersonic combustion with perfect transition in between both modes. Dual-mode scramjet engines are designed to operate in both subsonic combustion and supersonic combustion modes. The dual-mode scramjet engines generally have a constant area isolator followed by a combustor with a diverging area.

At low flight Mach number (Mach 3-5), the dual-mode combustion system operates in ram mode. A series of shock waves in the isolator decelerate and compress the flow entering the combustor to subsonic. At higher flight Mach numbers (greater than 5), the flow remains supersonic throughout the combustor and operates at scram mode.

If the heat released by the combustion process becomes greater than a critical value, the flow Mach number in the engine approaches unity in both supersonic and 
subsonic compressible flows. This is known as thermally choked flow [1]. This thermal choking may cause strong shocks at the engine inlet, introducing large amounts of drag at high flight Mach numbers and decreasing the performance of hypersonic engine, which is known as unstart process.

The thermal choking is referred to as Rayleigh flow if the area along the combustor is constant [115]. However, in the dual-mode combustors, the heat is released in a varying cross-sectional area. Designing the diverging area of the combustor for operability at wide range of conditions is a challenging procedure.

A new model is developed in this work which can predict the operability limit of dual-mode scramjet combustor at various flight conditions. Also the results from this model are compared with the observed data from the UVa dual-mode supersonic combustor facility (UVaSCF) experiments.

The variation of the cross-sectional area in the diverging combustor causes the Rayleigh flow equations to not apply. In this proposed work, a new model to calculate thermal choking has been developed for the analysis of non-constant area combustors. Based on the area ratio of the current combustor configuration, operability limits will be predicted for different flight Mach number conditions. Preliminary results (see section 4) indicated strong agreement with the operability limits from existing experimental results.

\subsection{Rayleigh Flow}

In nominally one-dimensional flow of the hypersonic combustor, the three most common factors tending to produce continuous changes in the state of a flowing stream are (1) changes in cross-sectional area, (2) wall friction, and (3) energy effects. The energy effect on the flow can be in form of external heat exchange, combustion, or moisture condensation [115]. In this particular study, combustion of the fuel-air mix- 
ture is the heat source to increase the stagnation temperature. The effects of change in chemical composition are small compared to heating effects due to small fuel-air ratio and therefore, it can be neglected.

The first case is isentropic flow, where the area changes without friction or energy exchange through the boundary. The second case corresponds to Fanno flow, where the wall frictions process is considered in the absence of area change and energy effects. The third case corresponds to the Rayleigh flow, where heat exchange occurs through the flow in a constant area, frictionless process. The schematic of all the three processes are shown in Figure 6.1.

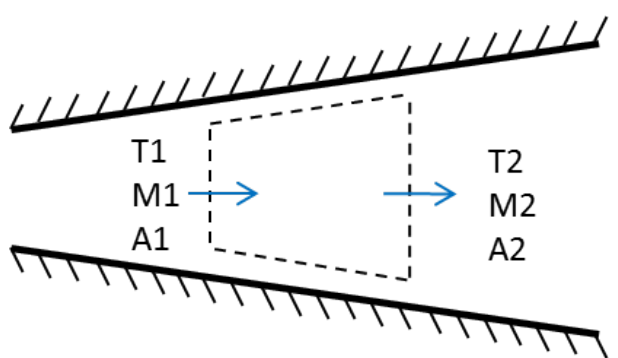

(a) Isentropic flow

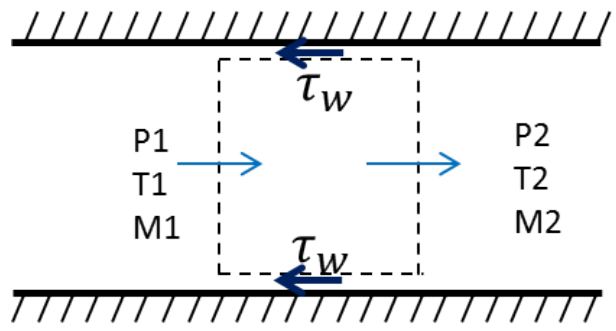

(b) Fanno flow

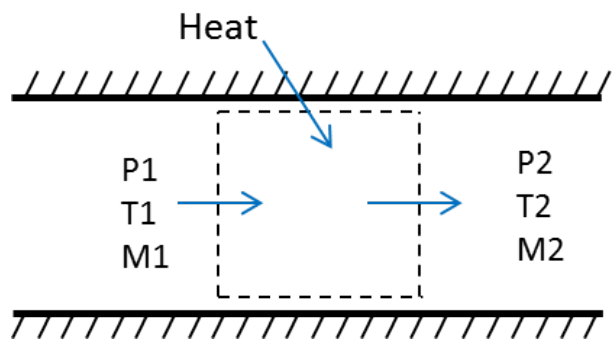

(c) Rayleigh flow

Figure 6.1: Schematic of the three one-dimensional flow cases with change in only one physical condition. 
All these cases are considered when one effect exists and the other two effects are absent. For the particular case of heating process of a flow in a constant area duct, the total temperature relation as a function of Mach number foe a perfect gas is given [115]

$$
\frac{T_{o_{2}}}{T_{o_{1}}}=\left(\left(\frac{M_{2}}{M_{1}}\right) \frac{1+\gamma M_{1}^{2}}{1+\gamma M_{2}^{2}}\right)^{2}\left(\frac{1+\frac{\gamma-1}{2} M_{2}^{2}}{1+\frac{\gamma-1}{2} M_{1}^{2}}\right)
$$

This relation neglects any area change in the flow field. However, in the dual-mode hypersonic combustor, the area is not constant. As shown in Figure 5.2, the height of the combustion region increases linearly with a constant diverging angle (about $3^{\circ}$ ). Therefore, the reported relation for total temperature is not valid for this study anymore. In this study, a new set of governing equations are proposed to include the area change effect in the heating process.

\subsection{Formulation of the Thermal Choking in a Vari- able Area}

\subsubsection{Quasi-One-Dimensional Flow Equations}

Consider the flow through the control volume shown in figure 6.2. The equations for the flow properties between sections 1 and 2 are as follows:

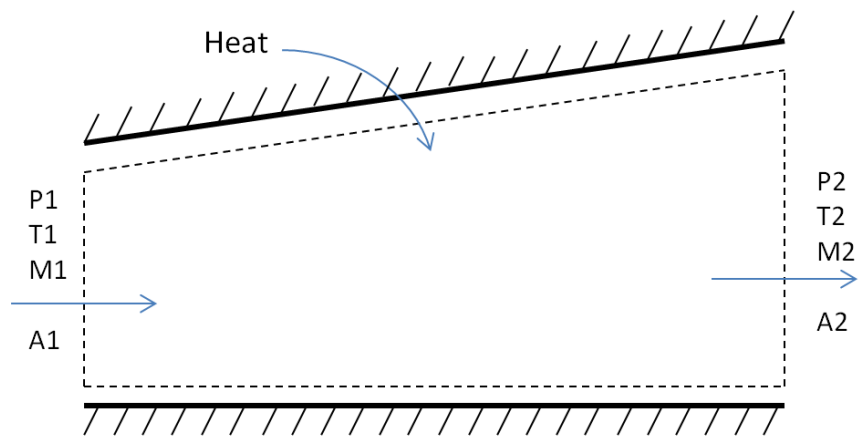

Figure 6.2: Control volume of heating a variable area flow. 
The continuity equation for this control volume is

$$
\rho_{1} u_{1} A_{1}=\rho_{2} u_{2} A_{2}
$$

The momentum equation assuming no friction is

$$
p_{1} A_{1}+\rho_{1} u_{1}^{2} A_{1}+\int_{A_{1}}^{A_{2}} p d A=p_{2} A_{2}+\rho_{2} u_{2}^{2} A_{2}
$$

The definition of Mach number for perfect gas is

$$
M=\frac{u}{\sqrt{\gamma R T}}
$$

Using the equation of state $p=\rho R T$ for the perfect gas gives

$$
\rho u^{2}=\gamma p M^{2}
$$

Combining Eqs. 6.5 and 6.3, gives

$$
p_{1} A_{1}+\gamma p_{1} M_{1}^{2} A_{1}+\int_{A_{1}}^{A_{2}} p d A=p_{2} A_{2}+\gamma p_{2} M_{2}^{2} A_{2}
$$




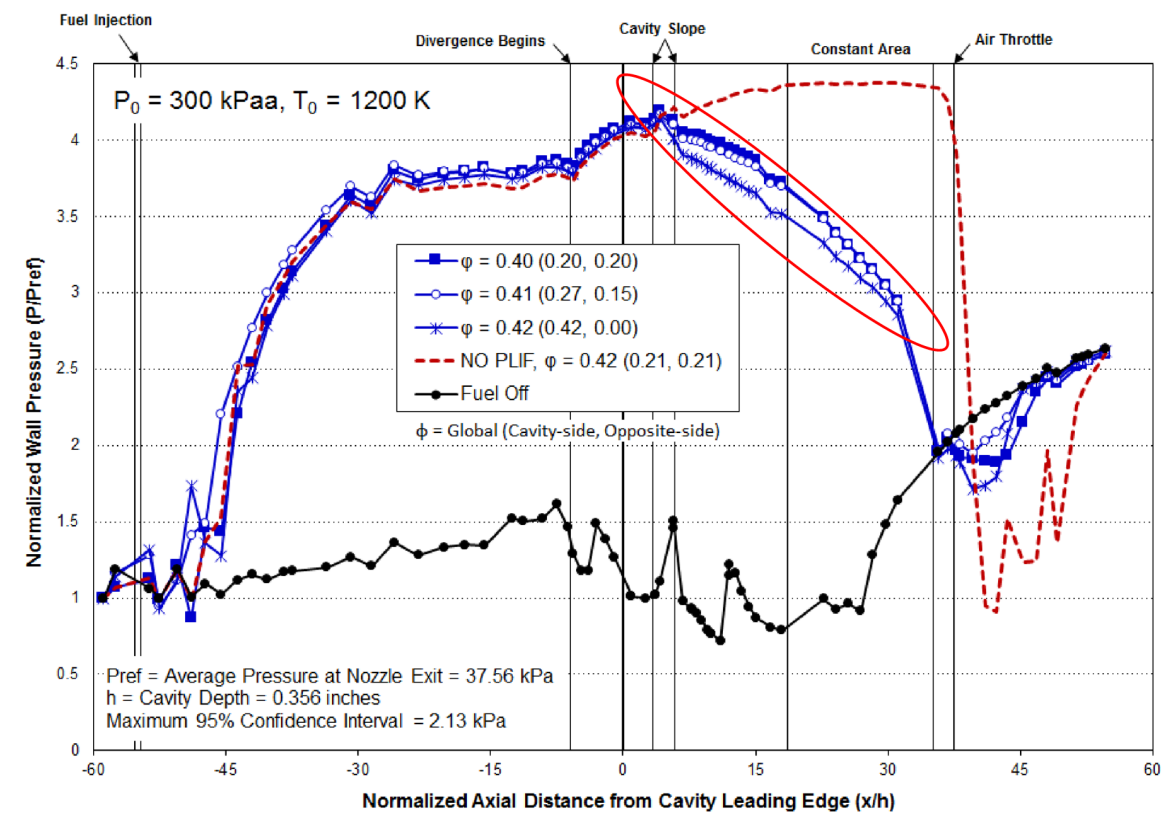

Figure 6.3: Normalized wall pressures along the dual-mode supersonic combustor with various fuel injection configurations [107].

To simplify the equation we assume the pressure profile varies linearly with respect to the cross sectional area. This assumption is in close agreement with the observed pressure measurements at the UVa dual-mode supersonic combustion experiments (see Figure 6.3). Therefore, the integral term in Eq. 6.6 is given by,

$$
\int_{A_{1}}^{A_{2}} p d A=\frac{p_{1}+p_{2}}{2}\left(A_{2}-A_{1}\right)
$$

Replacing the integral term in Eq. 6.6 gives

$$
p_{1} A_{1}+\gamma p_{1} M_{1}^{2} A_{1}+\frac{p_{1}+p_{2}}{2}\left(A_{2}-A_{1}\right)=p_{2} A_{2}+\gamma p_{2} M_{2}^{2} A_{2}
$$

By dividing both sides of the equation by $p_{1}$ and move all the terms with $p_{2} / p_{1}$ to one side, it yields

$$
\frac{p_{2}}{p_{1}}=\frac{\gamma M_{1}^{2} A_{1}+\frac{A_{1}+A_{2}}{2}}{\gamma M_{2}^{2} A_{2}+\frac{A_{1}+A_{2}}{2}}
$$


So the pressure ratio relation between the streams at the two sections is given by

$$
\frac{p_{2}}{p_{1}}=\left(\frac{A_{1}}{A_{2}}\right) \frac{\gamma M_{1}^{2}+0.5\left(1+\frac{A_{2}}{A_{1}}\right)}{\gamma M_{2}^{2}+0.5\left(1+\frac{A_{1}}{A_{2}}\right)}
$$

Using the ideal gas law $p=\rho R T$, the temperature ratio relation with pressure and density is

$$
\frac{T_{2}}{T_{1}}=\frac{p_{2} \rho_{1}}{p_{1} \rho_{2}}
$$

From the continuity equation 6.2 , the density ratio is given by

$$
\frac{\rho_{2}}{\rho_{1}}=\frac{u_{1} A_{1}}{u_{2} A_{2}}
$$

Inserting this into Eq. 6.11 gives

$$
\frac{T_{2}}{T_{1}}=\frac{p_{2} u_{2} A_{2}}{p_{1} u_{1} A_{1}}
$$

Using the definition of Mach number (Eq. 6.4), the ratio of the velocity of the flow at the two sections are given by

$$
\frac{u_{2}}{u_{1}}=\frac{M_{2}}{M_{1}} \sqrt{\frac{T_{2}}{T_{1}}}
$$

Using this relation in Eq. 6.13 yields

$$
\frac{T_{2}}{T_{1}}=\frac{p_{2} A_{2} M_{2}}{p_{1} A_{1} M_{1}} \sqrt{\frac{T_{2}}{T_{1}}}
$$

Rearranging and more simplification gives

$$
\frac{T_{2}}{T_{1}}=\left(\frac{p_{2} A_{2} M_{2}}{p_{1} A_{1} M_{1}}\right)^{2}
$$


Now, using the pressure relation from Eq. 6.10 and elimination of $p_{2} / p_{1}$ from Eq. 6.16 yields

$$
\frac{T_{2}}{T_{1}}=\left(\left(\frac{M_{2}}{M_{1}}\right) \frac{\gamma M_{1}^{2}+0.5\left(1+\frac{A_{2}}{A_{1}}\right)}{\gamma M_{2}^{2}+0.5\left(1+\frac{A_{1}}{A_{2}}\right)}\right)^{2}
$$

This equation give the relation for the general case of any flow condition at the two sections of the control volume. For the particular case where the heat addition to the stream is large enough that makes the flow at state 2 become sonic $\left(M_{2}=1\right)$, then the flow is called thermally choked. In this particular case, the properties at section 2 are denoted by asterisk, and the temperature relation is

$$
\frac{T^{*}}{T}=\left(\left(\frac{1}{M}\right) \frac{\gamma M^{2}+0.5\left(1+\frac{A^{*}}{A}\right)}{\gamma+0.5\left(1+\frac{A}{A^{*}}\right)}\right)^{2}
$$

This relation is showing the static temperature ratio of the choked flow condition to the initial inflow condition as a function of the initial Mach number and the area ratio between the two sections.

\subsubsection{Energy Conservation Equation in a Variable Area Ther- mally Choked Flow}

The energy equation for the control volume shown if Figure 6.2 is

$$
\dot{Q}+p_{1} u_{1} A_{1}+\rho_{1} u_{1} A_{1}\left(e_{1}+\frac{u_{1}^{2}}{2}\right)=p_{2} u_{2} A_{2}+\rho_{2} u_{2} A_{2}\left(e_{2}+\frac{u_{2}^{2}}{2}\right)
$$

where $\dot{Q}$ is the net rate of heat $(J / s)$ added to the control volume, $e$ is the internal energy per unit mass $(J / K g)$, and $\rho_{1} u_{1} A_{1}$ is the mass flow rate $(K g / s)$ through the

control volume. By dividing both sides of the equation by the mass flow rate and using conservation of mass (Eq. 6.2), we obtain

$$
\frac{\dot{Q}}{\rho_{1} u_{1} A_{1}}+\frac{p_{1}}{\rho_{1}}+e_{1}+\frac{u_{1}^{2}}{2}=\frac{p_{2}}{\rho_{2}}+e_{2}+\frac{u_{2}^{2}}{2}
$$


The ratio $\dot{Q} / \rho_{1} u_{1} A_{1}$ is simply the heat per unit mass, $q$, added to the control volume. By using the definition of enthalpy, $h=e+p v$, the energy equation becomes

$$
q+h_{1}+\frac{u_{1}^{2}}{2}=h_{2}+\frac{u_{2}^{2}}{2}
$$

The enthalpy for a perfect gas can be written as $h=c_{p} T$. Now we obtain

$$
q=\left(c_{p} T_{2}+\frac{u_{2}^{2}}{2}\right)-\left(c_{p} T_{1}+\frac{u_{1}^{2}}{2}\right)
$$

By using the definition of stagnation temperature $\left(T_{o}=T+u^{2} / 2 c_{p}\right)$, which corresponds to a state that the stream is adiabatically decelerated to zero velocity, the energy equation is related to stagnation temperature by

$$
q=c_{p}\left(T_{o_{2}}-T_{o_{1}}\right)
$$

For real gas where the heat capacity at constant pressure is not constant $\left(c_{p}\right.$ is a function of temperature), energy equation may be written as

$$
q=\int_{T_{o_{1}}}^{T_{o_{2}}} c_{p} d T
$$

Based on the definition of the stagnation temperature and Mach number, a relation between them is given by,

$$
T_{o}=T\left(1+\frac{\gamma-1}{2} M^{2}\right)
$$

Thus, the ratio of the total temperatures at two flow conditions are,

$$
\frac{T_{o_{2}}}{T_{o_{1}}}=\frac{T_{2}\left(1+\frac{\gamma-1}{2} M_{2}^{2}\right)}{T_{1}\left(1+\frac{\gamma-1}{2} M_{1}^{2}\right)}
$$

Combining Eqs. 6.26 and 6.17, the relation between the total temperatures at the 
two sections of the control volume flow is obtained

$$
\frac{T_{o_{2}}}{T_{o_{1}}}=\left(\left(\frac{M_{2}}{M_{1}}\right) \frac{\gamma M_{1}^{2}+0.5\left(1+\frac{A_{2}}{A_{1}}\right)}{\gamma M_{2}^{2}+0.5\left(1+\frac{A_{1}}{A_{2}}\right)}\right)^{2}\left(\frac{1+\frac{\gamma-1}{2} M_{2}^{2}}{1+\frac{\gamma-1}{2} M_{1}^{2}}\right)
$$

Equation 6.27 shows the relation of the total temperature change of the flow in terms of the Mach number change and the area change along the flow. In order to calculate the properties of the flow at thermally choked condition, the Mach number at state 2 is set equal to unity. The stagnation temperature change is calculated by obtaining the heat of reaction from the fuel-air mixture and using Eq. 6.23. By solving Eq. 6.27 for $A_{2} / A_{1}$, the critical area ratio which thermal choking happens, will be obtained. 


\subsection{Results and Discussion}

A computer program has been created in MATLAB in order to model the thermal choking in a variable area nozzle with combustion as the heat addition source. First the amount of heat produced from the combustion of the fuel-air mixture is calculated at the desired operating temperature and pressure using detailed chemistry model. This process is performed for a range of different equivalence ratios. Based on this calculation, the total temperature change can be found at the selected operating condition.

This program uses the calculated total temperature ratio as an input parameter and calculates the area ratio corresponding to the thermal choking limit from Eq. 6.27. Since this is a second order equation, the solution has two possible answers. One of the solutions has nonphysical value and is neglected. The other physical solution is considered the predicted area ratio of the thermal choking in the diverging nozzle of a combustor.

Figure 6.4 shows the calculated area ratio that thermal choking happens as a function of equivalence ratio for two combustion regimes, scramjet mode $(M=2)$ and ramjet mode $(\mathrm{M}=0.577)$. The fuel mixture is ethylene-air with equivalence ratios ranging from 0.1 to 1 . The equivalence ratio is equivalent to to amount of heat added to the compressible flow. As the equivalence increases, the enthalpy of reaction increases. The heat of reaction approaches the maximum at equivalence ratio of unity, similar to the adiabatic flame temperature.

In Figure 6.4, the dashed line is the model solution based on the method explained before. It is using the detailed chemical kinetic model (JetSurF2.0 [69]) with taking into account dissociation in order to calculate the thermal choking area ratio. On the other hand, the solid line is showing the solution from the model if a simple complete combustion occurs without dissociation. A simple reaction in the form of 
the following reaction is assumed.

$$
\phi C_{x} H_{y}+(x+y / 4)\left(O_{2}+3.76 N_{2}\right) \rightarrow a C O_{2}+b H_{2} O+c N_{2}+d O_{2}
$$

The stoichiometric coefficients of the reaction $(a, b, c, d)$ can be calculated based on the fuel molecular formula and the equivalence ratio $\phi$. When the equivalence ratio is less than unity, there is an excess oxygen which is not consumed in the fuel combustion $(d>0)$. By knowing the enthalpy of formation of the reactants and products (from reaction 6.28), and assuming a constant heat capacity for the mixture ( $c_{p}$ computed from Eq. 2.11), the temperature change of the fuel-air mixture can be calculated. The calculated temperature change is used in Eq. 6.27 to solve for the area ratio corresponding to thermal choking condition. The advantage of this simplified method is that the entire computer program can be done in MATLAB without any integration with a separate chemical kinetic solver. The first real gas method requires an integration of the detailed chemistry solver with the MATLAB code.

The results from this simplified model approximation match well with the results from the real combustion model with detailed kinetics for the lower range of equivalence ratio. For equivalence ratios of less than 0.5 , both models predict about the same thermal choking area ratio. This is due to the fact that the energy release of the reaction is not significantly high at low equivalence ratios. Therefore, the dissociation of the fuel-air combustion product mixture is not significant and can be neglected. However, as the equivalence ratio increases, the reaction heat release increases and the subsequent mixture high temperature results in significant dissociation of the mixture. As a result, the difference between the predictions from the two models are higher at higher equivalence ratios. 


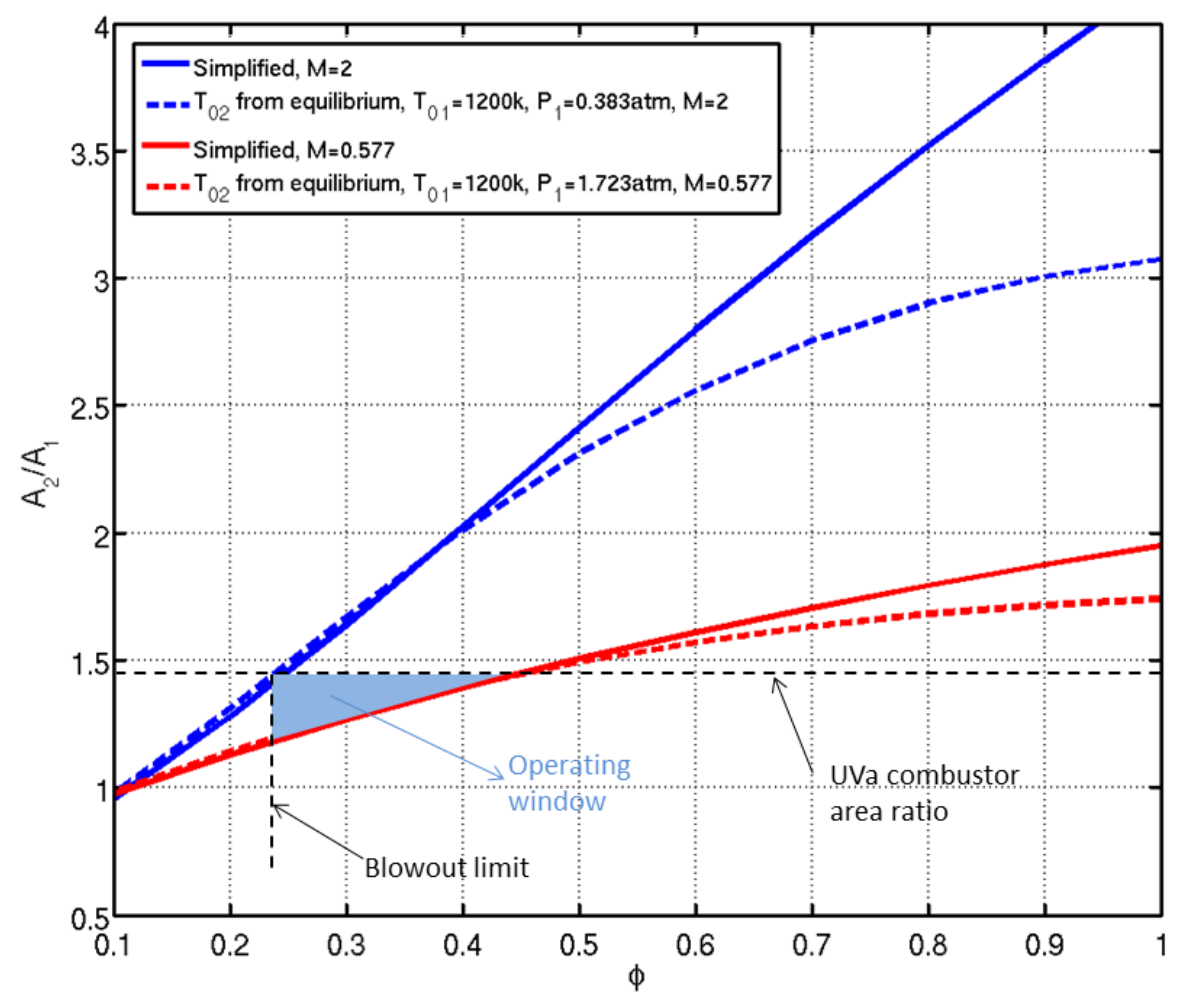

Figure 6.4: Area ratio curve vs. ethylene-air equivalence ratio at thermally choked condition. Solid line is using a simple model without chemical dissociation and constant $c_{p}$, dashed line is based on chemical equilibrium calculation (adiabatic) with full detailed chemistry and real gas thermodynamics.

The current diverging section area change is 1.44 for the UVa combustor. Based on the developed method in this study, the maximum ethylene equivalence ratio before thermal choking for the ram mode is about 0.45 . This number is exactly similar to what has been observed at UVaSCF experimental at the same operating conditions. Similarly, at other extreme condition where the combustion takes place in the scram mode, the maximum ethylene equivalence ratio is about 0.23 resulted from this computational model, which is the same number the experiments have reported. This model's accurate prediction of the hypersonic combustor operating limits proves that this model is a computationally efficient useful tool for designing and improving hypersonic combustors with identification of their operating limits at a wide range of flight conditions. The most important advantages of this method is that it eliminates 
any requirement for time consuming multi-dimensional reacting flow simulations.

\subsection{Summary}

A numerical model was developed to predict the thermal choking in a variable area (diverging nozzle) in dual-mode supersonic combustor. The model was used in order to find the operability limits of ethylene fuel combustion at the relevant conditions of the UVa supersonic combustion facility.

The prediction from this simple model matched very well with the observed experimental operability limits. This novel computational model eliminated the need to perform a computationally expensive 3D reacting flow simulation in order to find the thermal choking limitations. 


\section{Chapter 7}

\section{Summary and Future Works}

The present work investigated three major problems in hypersonic airbreathing propulsion systems: 1) Endothermic fuel pyrolysis in cooling channels of hypersonic combustors. 2) Flame holding and stabilization of fuel-air mixture oxidation in the combustor at hypersonic speeds with short residence time. 3) Thermal choking and operability limits of hypersonic combustors with diverging nozzle. The first three chapters provide a comprehensive analysis for objective 1. Four different hydrocarbon fuels (i.e., ethane, n-butane, n-dodecane, and JP-8) were considered for this analysis.

In chapter 2, endothermic fuel pyrolysis in a cooling channel was investigated based on experiments performed in a tubular microflow reactor developed at UVa Combustion and Energy Lab. The studied tube reactor is a simplified representation of real cooling channels at hypersonic combustors. First, ethane pyrolysis was considered as a test case to evaluate the flow reactor. In the analysis of ethane pyrolysis, three detailed chemical kinetic mechanisms were compared against the experimental data. The results for the major species had reasonable agreement between all the models. However, it was observed that the minor species mole fraction profiles had significant disagreement between the models. AramcoMech2.0 model showed better agreement with the experimental data for most of the measured species compared to 
other two models. Before generalization to larger hydrocarbon molecules, n-butane was considered as another test fuel. In the analysis of n-butane pyrolysis, the predictions from three detailed chemical kinetic mechanisms were compared against the experimental data. All the three chemical kinetic models predicted most of the species profiles with large discrepancies. None of the models showed good agreement with the data for all of the measured species. This led to further investigations using sensitivity and uncertainty analysis in order to identify the target parameters that are most important to study more for improvement and optimization. The details of this analysis were presented in chapter 3. Finally, as a large hydrocarbon molecule and a common surrogate for jet fuels, the pyrolysis of n-dodecane was investigated in the flow reactor. The predictions from four chemical kinetic models were compared with the experimental data. Mze-Ahmed et al. model predicted most of the major species with good agreement. Also a simplified lumped one-step reaction model was developed for JP-8 pyrolysis. The results from this model and two other available detailed kinetic models were compared against the experimental data. The predictions from this developed simple model matched well with the experimental data for most of the major species.

In chapter 3 , a complete sensitivity analysis and uncertainty quantification was performed for n-dodecane, ethane, and n-butane pyrolysis. Sensitivity analysis and reaction pathway analysis were performed to identify the important reactions contributing to each fuel pyrolysis pathways and governing the major species mole fractions at the corresponding conditions. Based on these findings, a computationally expensive Monte Carlo simulation with a a large sample size was performed in order to quantify the model uncertainty. The results were compared with the experimental data and measurements uncertainty. Results showed that the uncertainties from the chemical models are much higher than the experimental uncertainties. Thus, chemical kinetic model optimization is needed in order to reduce the uncertainties 
associated with the existing models. Furthermore, based on the analysis performed in this chapter, a reduced reaction mechanism was developed for n-dodecane pyrolysis to be used in multi-dimensional reacting flow simulations. The predictions from the reduced model were compared with the detailed model and showed less than $2 \%$ error for models with 50 species or more.

In Chapter 4, the two-dimensional effects of the laminar parabolic flow in the tubular reactor were investigated. A complete numerical axi-symmetric Navier-Stokes simulation including chemical reactions was performed in order to understand the stratification issue and radial gradient effects associated with the parabolic velocity profile. The numerical calculation is based on the boundary conditions from the UVa tube reactor experiment. The simulation results showed significant radial variation in the highly reactive region (0-30 ms residence time in the studied case). The radial gradients remain high for the larger species $(\mathrm{C} 7-\mathrm{C} 12)$ for the entire region, but the smaller species with faster diffusivity become radially uniform by the end of the reactor. The results from the two-dimensional numerical simulation of n-dodecane pyrolysis were compared with the one-dimensional plug flow simulation results. Even though large radial gradients were present in the tube reactor, the difference between the cross-sectional averaged profiles from $2 \mathrm{D}$ results and the one-dimensional plug flow results were negligible.

In Chapter 5, a new approach to model the fuel oxidation in a dual-mode supersonic combustor was investigated. The cavity flame holder and the turbulent mixing and reaction interaction in the combustor were simulated by a computationally efficient implementation of partially stirred reactor model in connection with perfectly stirred reactor. The relevant time scales for hypersonic combustors were used for the simulations. The ignition delay time predictions from this analysis had good agreement with the observed experimental flame holding results. This simplified method was recently used to design a new insert cavity flame holder for improved combustion 
performance in the supersonic combustor. A reduced reaction mechanism for ethylene combustion was also developed. The predictions from this reduced model were validated for a range applicable to hypersonic conditions.

In Chapter 6, a numerical model was developed to predict the thermal choking in the variable area (diverging nozzle) of dual-mode supersonic combustors. The model was used in order to find the operability limits of ethylene fuel combustion at the UVa supersonic combustion facility. The prediction from this simple model matched very well with the observed experimental operability limits. This novel computational model eliminated the need to perform a computationally expensive 3D reacting flow simulation in order to find the thermal choking limitations.

For future work, JP-10 (exo-tetrahydrodicyclopentadiene) can be the focus of endothermic fuel pyrolysis analysis. JP-10 is a single-component fuel, unlike other aviation fuels which are a blend of different components. Significant ongoing research is focused on using JP-10 for hypersonic air-breathing propulsion systems. The main advantages of JP-10 as a fuel are high volumetric energy density, low freezing point, and good heat transfer properties. A detailed model with a large number of species and reactions is recently developed by Green research group at MIT using Reaction Mechanism Generator (RMG) for JP-10 combustion and pyrolysis. This model can be used in order to develop a reduced model applicable for multi-dimensional reacting flow simulation in hypersonic applications.

Also in the future, the simplified lumped reaction model for JP-8 pyrolysis will be integrated with a detailed oxidation chemistry sub-mechanism suitable for the products of JP-8 decomposition. For soot studies it will include the aromatic species and reactions. Then the complete model will be validated against various fuel oxidation experiments such as shock tube ignition delay experiments, flow reactors, flame speed measurements, and jet-stirred reactors. 


\section{Appendix A}

\section{Reduced Ethylene-Air Reaction Mechanism}

This section shows the reduced ethylene oxidation mechanism developed in this work. It consists of 26 species and 132 elementary reactions. It must be noted that the units for this mechanism are in moles, centimeters, seconds, Kelvin, and calories. The units of activation energy (E) are in calories per mole. The units of pre-exponential factor (A) for each reaction can be determined by the order of the reaction and requires dimensional analysis. 


$\begin{array}{ll}\text { ELEMENTS } & \text { ATOMIC } \\ \text { CONSIDERED } & \text { WEIGHT } \\ & \\ \text { 1. } \mathrm{O} & 15.9994 \\ 2 . \mathrm{H} & 1.00797 \\ 3 . \mathrm{C} & 12.0112 \\ 4 . \mathrm{N} & 14.0067 \\ - & \end{array}$

\section{C}

P H

H A

A $R$

SPECIES

S G MOLECULAR TEMPERATURE ELEMENT COUNT

CONSIDERED

$\begin{array}{llllllll}\text { E E WEIGHT LOW HIGH } & \mathrm{O} & \mathrm{H} & \mathrm{C} & \mathrm{N} & \mathrm{AR}\end{array}$

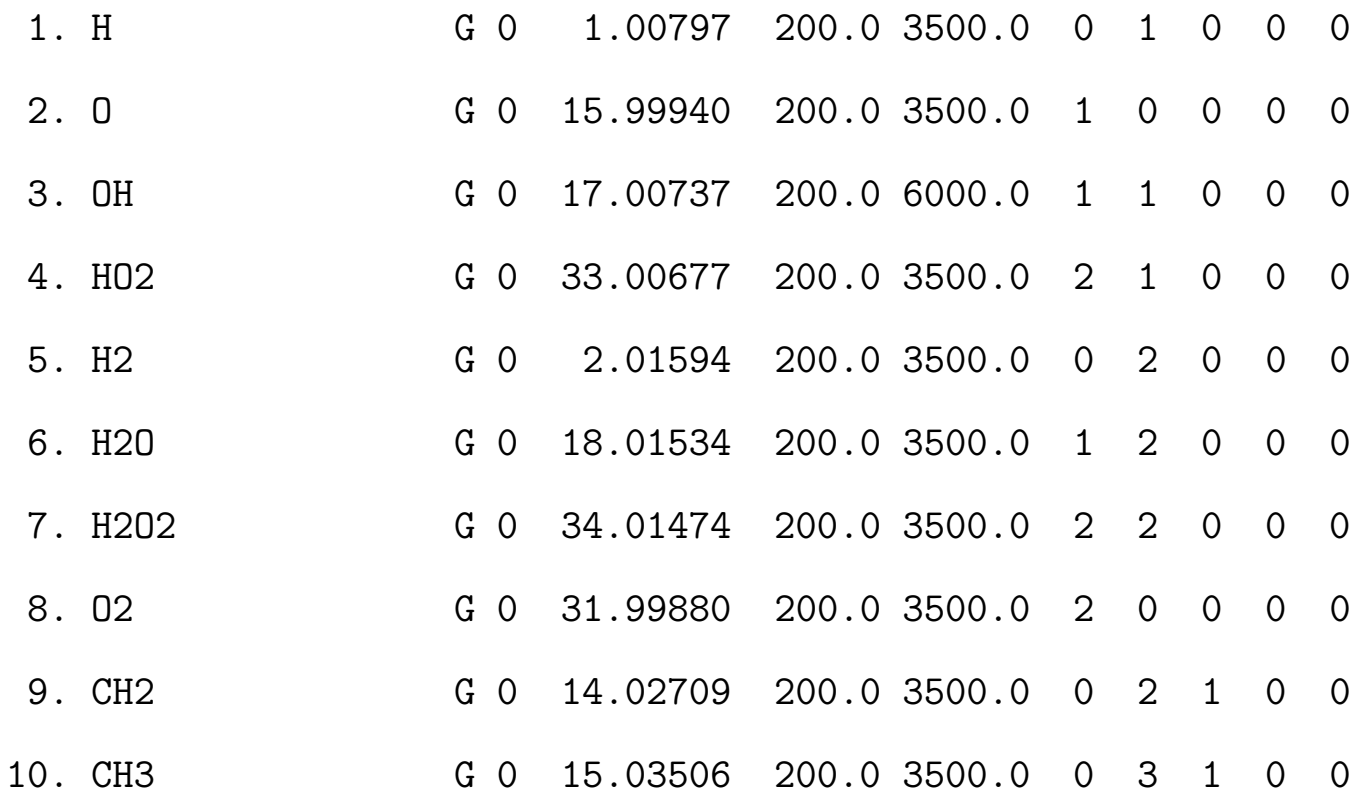




\begin{tabular}{|c|c|c|c|c|c|c|c|c|}
\hline 11. $\mathrm{HCO}$ & G & 29.01852 & 200.0 & 3500.0 & 1 & 1 & 1 & 0 \\
\hline 12. $\mathrm{CH} 2 \mathrm{O}$ & G & 30.02649 & 200.0 & 3500.0 & 1 & 2 & 1 & 0 \\
\hline 13. СН30 & G & 31.03446 & 200.0 & 6000.0 & 1 & 3 & 1 & 0 \\
\hline 14. CO & G & 28.01055 & 200.0 & 3500.0 & 1 & 0 & 1 & 0 \\
\hline 15. CO2 & G & 44.00995 & 200.0 & 3500.0 & 2 & 0 & 1 & 0 \\
\hline 16. $\mathrm{C} 2 \mathrm{H} 2$ & G & 26.03824 & 200.0 & 3500.0 & 0 & 2 & 2 & 0 \\
\hline 17. $\mathrm{H} 2 \mathrm{CC}$ & G & 26.03824 & 200.0 & 6000.0 & 0 & 2 & 2 & 0 \\
\hline 18. С2H3 & G & 27.04621 & 200.0 & 3500.0 & 0 & 3 & 2 & 0 \\
\hline 19. $\mathrm{C} 2 \mathrm{H} 4$ & G & 28.05418 & 200.0 & 3500.0 & 0 & 4 & 2 & 0 \\
\hline 20. $\mathrm{C} 2 \mathrm{H} 5$ & G & 29.06215 & 200.0 & 3500.0 & 0 & 5 & 2 & 0 \\
\hline 21. $\mathrm{C} 2 \mathrm{H} 6$ & G & 30.07012 & 200.0 & 3500.0 & 0 & 6 & 2 & 0 \\
\hline 22. $\mathrm{HCCO}$ & G & 41.02967 & 300.0 & 4000.0 & 1 & 1 & 2 & 0 \\
\hline 23. $\mathrm{CH} 2 \mathrm{CO}$ & G & 42.03764 & 200.0 & 3500.0 & 1 & 2 & 2 & 0 \\
\hline 24. $\mathrm{CH} 2 \mathrm{CHO}$ & G & 43.04561 & 300.0 & 5000.0 & 1 & 3 & 2 & 0 \\
\hline 25. $\mathrm{CH} 2 \mathrm{OCH} 2$ & G & 44.05358 & 298.1 & 3000.0 & 1 & 4 & 2 & 0 \\
\hline 26. N2 & G & 28.01340 & 300.0 & 5000.0 & 0 & 0 & 0 & 2 \\
\hline
\end{tabular}

$(\mathrm{k}=\mathrm{A} T * * \mathrm{~b} \exp (-\mathrm{E} / \mathrm{RT}))$

REACTIONS CONSIDERED

A $\quad$ b $\quad$ E

1. $\mathrm{H}+\mathrm{O} 2<=>\mathrm{O}+\mathrm{OH}$

$2.64 \mathrm{E}+16 \quad-0.7 \quad 17041.0$

2. $\mathrm{O}+\mathrm{H} 2<=>\mathrm{H}+\mathrm{OH}$

$\begin{array}{lll}4.59 \mathrm{E}+04 & 2.7 & 6260.0\end{array}$

3. $\mathrm{OH}+\mathrm{H} 2<=>\mathrm{H}+\mathrm{H} 2 \mathrm{O}$

$\begin{array}{lll}1.73 E+08 & 1.5 & 3430.0\end{array}$

4. $2 \mathrm{OH}<=>\mathrm{O}+\mathrm{H} 2 \mathrm{O}$

$\begin{array}{lll}3.97 \mathrm{E}+04 & 2.4 & -2110.0\end{array}$

5. $2 \mathrm{H}+\mathrm{M}<=>\mathrm{H} 2+\mathrm{M}$

$1.78 \mathrm{E}+18-1.0$

0.0 


$\begin{array}{lll}\text { H2 } & \text { Enhanced by } & 0.000 \mathrm{E}+00 \\ \mathrm{H} 20 & \text { Enhanced by } & 0.000 \mathrm{E}+00 \\ \mathrm{C} 22 & \text { Enhanced by } & 0.000 \mathrm{E}+00\end{array}$

6. $2 \mathrm{H}+\mathrm{H} 2<=>2 \mathrm{H} 2$

$9.00 \mathrm{E}+16 \quad-0.6$

0.0

7. $2 \mathrm{H}+\mathrm{H} 2 \mathrm{O}<=>\mathrm{H} 2+\mathrm{H} 2 \mathrm{O}$

5. $62 \mathrm{E}+19-1.2$

0.0

8. $2 \mathrm{H}+\mathrm{CO} 2<=>\mathrm{H} 2+\mathrm{CO} 2$

$5.50 \mathrm{E}+20-2.0$

0.0

9. $\mathrm{H}+\mathrm{OH}+\mathrm{M}<=>\mathrm{H} 2 \mathrm{O}+\mathrm{M}$

$4.40 \mathrm{E}+22-2.0$

0.0

\section{$\mathrm{H} 2$}

H2O

CO

$\mathrm{CO} 2$

10. $\mathrm{O}+\mathrm{H}+\mathrm{M}<=>\mathrm{OH}+\mathrm{M}$

$\mathrm{H} 2$

$\mathrm{H} 2 \mathrm{O}$

$\mathrm{CO2}$

11. $20+M<=>02+M$

$\mathrm{H} 2$

$\mathrm{H} 2 \mathrm{O}$

$\mathrm{CO2}$
Enhanced by $2.000 \mathrm{E}+00$

Enhanced by $6.300 \mathrm{E}+00$

Enhanced by $1.750 \mathrm{E}+00$

Enhanced by

$3.600 \mathrm{E}+00$

$9.43 \mathrm{E}+18-1.0$

0.0
Enhanced by $2.000 \mathrm{E}+00$

Enhanced by 1.200E+01

Enhanced by $3.600 \mathrm{E}+00$

1. $20 \mathrm{E}+17-1.0 \quad 0.0$

$\begin{array}{ll}\text { Enhanced by } & 2.400 \mathrm{E}+00 \\ \text { Enhanced by } & 1.540 \mathrm{E}+01 \\ \text { Enhanced by } & 3.600 \mathrm{E}+00\end{array}$

12. $\mathrm{H}+\mathrm{O} 2(+\mathrm{M})<=>\mathrm{HO} 2(+\mathrm{M})$

$5.12 \mathrm{E}+12 \quad 0.4$

0.0 Low pressure limit:

$0.63280 E+20-0.14000 E+01$

$0.00000 \mathrm{E}+00$

TROE centering:

$0.50000 \mathrm{E}+00$

$0.10000 \mathrm{E}-29$

$0.10000 \mathrm{E}+31$

$\mathrm{H} 2 \mathrm{O}$

Enhanced by

1. 189E+01

CO

Enhanced by

$1.090 \mathrm{E}+00$

CO2

Enhanced by

$2.180 \mathrm{E}+00$

13. $\mathrm{H} 2+02<=>\mathrm{HO} 2+\mathrm{H}$

14. $2 \mathrm{OH}(+\mathrm{M})<=>\mathrm{H} 2 \mathrm{O} 2(+\mathrm{M})$
$5.92 \mathrm{E}+05 \quad 2.4$

53502.0

1. $11 \mathrm{E}+14-0.4$

0.0 
Low pressure limit: $\quad 0.20100 \mathrm{E}+18-0.58400 \mathrm{E}+00-0.22930 \mathrm{E}+04$

$\begin{array}{ccc}\text { TROE centering: } & 0.73460 \mathrm{E}+00 & 0.94000 \mathrm{E}+02 \\ \text { H2 } & \text { Enhanced by } & 2.000 \mathrm{E}+00 \\ \text { H2O } & \text { Enhanced by } & 6.000 \mathrm{E}+00 \\ \text { CO } & \text { Enhanced by } & 1.750 \mathrm{E}+00 \\ \text { CO2 } & \text { Enhanced by } & 3.600 \mathrm{E}+00\end{array}$

15. $\mathrm{HO} 2+\mathrm{H}<=>0+\mathrm{H} 2 \mathrm{O}$

16. $\mathrm{HO} 2+\mathrm{H}<=>2 \mathrm{OH}$

17. $\mathrm{HO} 2+\mathrm{O}<=>\mathrm{OH}+\mathrm{O} 2$

18. $2 \mathrm{HO} 2<=>02+\mathrm{H} 2 \mathrm{O} 2$

Declared duplicate reaction...

19. $2 \mathrm{HO} 2<=>02+\mathrm{H} 2 \mathrm{O} 2$

Declared duplicate reaction...

20. $\mathrm{HO} 2+\mathrm{OH}<=>\mathrm{H} 2 \mathrm{O}+\mathrm{O} 2$

21. $\mathrm{H} 2 \mathrm{O} 2+\mathrm{H}<=>\mathrm{HO} 2+\mathrm{H} 2$

22. $\mathrm{H} 2 \mathrm{O} 2+\mathrm{H}<=>\mathrm{OH}+\mathrm{H} 2 \mathrm{O}$

23. $\mathrm{H} 2 \mathrm{O} 2+\mathrm{O}<=>\mathrm{OH}+\mathrm{HO} 2$

24. $\mathrm{H} 2 \mathrm{O} 2+\mathrm{OH}<=>\mathrm{HO} 2+\mathrm{H} 2 \mathrm{O}$

Declared duplicate reaction...

25. $\mathrm{H} 2 \mathrm{O} 2+\mathrm{OH}<=>\mathrm{HO} 2+\mathrm{H} 2 \mathrm{O}$

Declared duplicate reaction...

26. $\mathrm{CO}+\mathrm{O}(+\mathrm{M})<=>\mathrm{CO} 2(+\mathrm{M})$

1. $36 \mathrm{E}+10$

0.0

2384.0

Low pressure limit: $0.11730 \mathrm{E}+25-0.27900 \mathrm{E}+01$

$0.41910 \mathrm{E}+04$

$\begin{array}{lll}\text { H2 } & \text { Enhanced by } & 2.000 \mathrm{E}+00 \\ \mathrm{H} 20 & \text { Enhanced by } & 1.200 \mathrm{E}+01 \\ \mathrm{C} 2 & \text { Enhanced by } & 3.600 \mathrm{E}+00\end{array}$

27. $\mathrm{CO}+\mathrm{OH}<=>\mathrm{CO} 2+\mathrm{H}$

$\begin{array}{lll}3.97 \mathrm{E}+12 & 0.0 & 671.0\end{array}$

$7.48 \mathrm{E}+13 \quad 0.0 \quad 295.0$

$\begin{array}{lll}4.00 \mathrm{E}+13 & 0.0 & 0.0\end{array}$

$\begin{array}{lll}1.30 E+11 & 0.0 & -1630.0\end{array}$

$3.66 \mathrm{E}+14 \quad 0.0 \quad 12000.0$

$2.89 \mathrm{E}+13 \quad 0.0 \quad-500.0$

$6.05 \mathrm{E}+06 \quad 2.0 \quad 5200.0$

$2.41 \mathrm{E}+13 \quad 0.0 \quad 3970.0$

$9.63 \mathrm{E}+06 \quad 2.0 \quad 3970.0$

$2.00 \mathrm{E}+12 \quad 0.0 \quad 427.0$

$2.67 \mathrm{E}+41 \quad-7.0 \quad 37600.0$ 
Declared duplicate reaction...

28. $\mathrm{CO}+\mathrm{OH}<=>\mathrm{CO} 2+\mathrm{H}$

$\begin{array}{lll}5.76 E+12 & -0.7 & 331.8\end{array}$

Declared duplicate reaction...

29. $\mathrm{CO}+02<=>\mathrm{CO} 2+0$

$1.12 \mathrm{E}+12 \quad 0.0 \quad 47700.0$

30. $\mathrm{CO}+\mathrm{HO} 2<=>\mathrm{CO} 2+\mathrm{OH}$

$\begin{array}{lll}1.57 \mathrm{E}+05 & 2.2 & 17942.6\end{array}$

31. $\mathrm{HCO}+\mathrm{H}<=>\mathrm{CO}+\mathrm{H} 2$

$\begin{array}{lll}1.20 \mathrm{E}+14 & 0.0 & 0.0\end{array}$

32. $\mathrm{HCO}+\mathrm{O}<=>\mathrm{CO}+\mathrm{OH}$

$\begin{array}{lll}3.00 \mathrm{E}+13 & 0.0 & 0.0\end{array}$

33. $\mathrm{HCO}+\mathrm{O}<=>\mathrm{CO} 2+\mathrm{H}$

$\begin{array}{lll}3.00 E+13 & 0.0 & 0.0\end{array}$

34. $\mathrm{HCO}+\mathrm{OH}<=>\mathrm{CO}+\mathrm{H} 2 \mathrm{O}$

$\begin{array}{lll}3.02 \mathrm{E}+13 & 0.0 & 0.0\end{array}$

35. $\mathrm{HCO}+\mathrm{M}<=>\mathrm{CO}+\mathrm{H}+\mathrm{M}$

$1.87 \mathrm{E}+17 \quad-1.0 \quad 17000.0$

$\begin{array}{lll}\text { H2 } & \text { Enhanced by } & 2.000 \mathrm{E}+00 \\ \mathrm{H} 20 & \text { Enhanced by } & 0.000 \mathrm{E}+00 \\ \mathrm{CO} & \text { Enhanced by } & 1.750 \mathrm{E}+00 \\ \mathrm{C0} 2 & \text { Enhanced by } & 3.600 \mathrm{E}+00\end{array}$

36. $\mathrm{HCO}+\mathrm{H} 2 \mathrm{O}<=>\mathrm{CO}+\mathrm{H}+\mathrm{H} 2 \mathrm{O}$

$2.24 \mathrm{E}+18-1.0 \quad 17000.0$

37. $\mathrm{HCO}+02<=>\mathrm{CO}+\mathrm{HO} 2$

$\begin{array}{lll}1.20 \mathrm{E}+10 & 0.8 & -727.0\end{array}$

38. $\mathrm{CO}+\mathrm{H} 2(+\mathrm{M})<=>\mathrm{CH} 2 \mathrm{O}(+\mathrm{M})$

$\begin{array}{lll}4.30 \mathrm{E}+07 & 1.5 & 79600.0\end{array}$

Low pressure limit: $\quad 0.50700 \mathrm{E}+28-0.34200 \mathrm{E}+01$

$0.84350 E+05$

TROE centering:

$0.93200 \mathrm{E}+00 \quad 0.19700 \mathrm{E}+03$

$0.15400 \mathrm{E}+04$

$0.10300 \mathrm{E}+05$

$\mathrm{H} 2$

Enhanced by $2.000 \mathrm{E}+00$

$\mathrm{H} 2 \mathrm{O}$

Enhanced by $6.000 \mathrm{E}+00$

CO

Enhanced by $1.500 \mathrm{E}+00$

$\mathrm{CO2}$

Enhanced by $2.000 \mathrm{E}+00$

$\mathrm{C} 2 \mathrm{H} 6$

Enhanced by $3.000 \mathrm{E}+00$

39. $\mathrm{HCO}+\mathrm{H}(+\mathrm{M})<=>\mathrm{CH} 2 \mathrm{O}(+\mathrm{M})$

$\begin{array}{lll}1.09 \mathrm{E}+12 & 0.5 & -260.0\end{array}$

Low pressure limit:

$0.13500 \mathrm{E}+25-0.25700 \mathrm{E}+01$

$0.14250 \mathrm{E}+04$

TROE centering:

$0.78240 \mathrm{E}+00 \quad 0.27100 \mathrm{E}+03$

$0.27550 \mathrm{E}+04$

$0.65700 E+04$ 


$\begin{array}{lll}\mathrm{H} 2 & \text { Enhanced by } & 2.000 \mathrm{E}+00 \\ \mathrm{H} 20 & \text { Enhanced by } & 6.000 \mathrm{E}+00 \\ \mathrm{CO} & \text { Enhanced by } & 1.500 \mathrm{E}+00 \\ \mathrm{CO} 2 & \text { Enhanced by } & 2.000 \mathrm{E}+00 \\ \mathrm{C} 2 \mathrm{H} 6 & \text { Enhanced by } & 3.000 \mathrm{E}+00\end{array}$

40. $\mathrm{CH} 2+\mathrm{H}(+\mathrm{M})<=>\mathrm{CH} 3(+\mathrm{M})$

$\begin{array}{lll}2.50 \mathrm{E}+16 & -0.8 & 0.0\end{array}$

Low pressure limit:

$0.32000 \mathrm{E}+28-0.31400 \mathrm{E}+01$

$0.12300 \mathrm{E}+04$

TROE centering:

$0.68000 \mathrm{E}+00 \quad 0.78000 \mathrm{E}+02$

$0.19950 \mathrm{E}+04 \quad 0.55900 \mathrm{E}+04$

H2

Enhanced by $2.000 \mathrm{E}+00$

$\mathrm{H} 2 \mathrm{O}$

Enhanced by $6.000 \mathrm{E}+00$

$\mathrm{CO}$

Enhanced by

1. $500 \mathrm{E}+00$

$\mathrm{CO} 2$

Enhanced by

$2.000 \mathrm{E}+00$

$\mathrm{C} 2 \mathrm{H} 6$

Enhanced by

$3.000 \mathrm{E}+00$

41. $\mathrm{CH} 2+\mathrm{O}<=>\mathrm{HCO}+\mathrm{H}$

8. $00 \mathrm{E}+13 \quad 0.0$

0.0

42. $\mathrm{CH} 2+\mathrm{OH}<=>\mathrm{CH} 2 \mathrm{O}+\mathrm{H}$

$2.00 \mathrm{E}+13 \quad 0.0$

0.0

43. $\mathrm{CH} 2+\mathrm{H} 2<=>\mathrm{H}+\mathrm{CH} 3$

$5.00 \mathrm{E}+05$

2.0

7230.0

44. $\mathrm{CH} 2+\mathrm{O} 2<=>\mathrm{HCO}+\mathrm{OH}$

$\begin{array}{lll}1.06 \mathrm{E}+13 & 0.0 & 1500.0\end{array}$

45. $\mathrm{CH} 2+\mathrm{O} 2<=>\mathrm{CO} 2+2 \mathrm{H}$

$2.64 \mathrm{E}+12$

0.0

1500.0

46. $\mathrm{CH} 2+\mathrm{HO} 2<=>\mathrm{CH} 2 \mathrm{O}+\mathrm{OH}$

$2.00 \mathrm{E}+13 \quad 0.0$

0.0

47. $\mathrm{CH} 2+\mathrm{CO}(+\mathrm{M})<=>\mathrm{CH} 2 \mathrm{CO}(+\mathrm{M})$

$\begin{array}{lll}8.10 \mathrm{E}+11 & 0.5 & 4510.0\end{array}$

Low pressure limit:

$0.26900 \mathrm{E}+34-0.51100 \mathrm{E}+01$

$0.70950 \mathrm{E}+04$

TROE centering:

$0.59070 \mathrm{E}+00$

$0.27500 \mathrm{E}+03$

$0.12260 \mathrm{E}+04$

$0.51850 \mathrm{E}+04$

$\mathrm{H} 2$

Enhanced by $2.000 \mathrm{E}+00$

$\mathrm{H} 2 \mathrm{O}$

Enhanced by

$6.000 \mathrm{E}+00$

CO

Enhanced by

$1.500 \mathrm{E}+00$

CO2

Enhanced by

$2.000 E+00$

$\mathrm{C} 2 \mathrm{H} 6$

Enhanced by

$3.000 \mathrm{E}+00$ 


\begin{tabular}{|c|c|c|c|c|c|c|}
\hline 48. & $2 \mathrm{CH} 2<=>\mathrm{C} 2 \mathrm{H} 2+\mathrm{H} 2$ & & & $3.20 \mathrm{E}+13$ & 0.0 & 0.0 \\
\hline 49 . & $\mathrm{CH} 2 \mathrm{O}+\mathrm{H}(+\mathrm{M})<=>\mathrm{CH} 3 \mathrm{O}(+\mathrm{N}$ & & & $5.40 \mathrm{E}+11$ & 0.5 & 2600.0 \\
\hline & Low pressure limit: & $0.22000 \mathrm{E}+31$ & $-0.48000 \mathrm{E}+01$ & $0.55600 \mathrm{E}+04$ & & \\
\hline & TROE centering: & $0.75800 \mathrm{E}+00$ & $0.94000 \mathrm{E}+02$ & $0.15550 \mathrm{E}+04$ & & $.42000 \mathrm{E}+04$ \\
\hline & H2 & Enhanced by & $2.000 \mathrm{E}+00$ & & & \\
\hline & $\mathrm{H} 2 \mathrm{O}$ & Enhanced by & $6.000 \mathrm{E}+00$ & & & \\
\hline & $\mathrm{CO}$ & Enhanced by & $1.500 \mathrm{E}+00$ & & & \\
\hline & $\mathrm{C} 02$ & Enhanced by & $2.000 \mathrm{E}+00$ & & & \\
\hline & $\mathrm{C} 2 \mathrm{H} 6$ & Enhanced by & $3.000 \mathrm{E}+00$ & & & \\
\hline 50. & $\mathrm{CH} 2 \mathrm{O}+\mathrm{H}<=>\mathrm{HCO}+\mathrm{H} 2$ & & & $2.30 \mathrm{E}+10$ & 1.1 & 3275.0 \\
\hline 51. & $\mathrm{CH} 2 \mathrm{O}+\mathrm{O}<=>\mathrm{HCO}+\mathrm{OH}$ & & & $3.90 \mathrm{E}+13$ & 0.0 & 3540.0 \\
\hline 52. & $\mathrm{CH} 2 \mathrm{O}+\mathrm{OH}<=>\mathrm{HCO}+\mathrm{H} 2 \mathrm{O}$ & & & $3.43 E+09$ & 1.2 & -447.0 \\
\hline 53. & $\mathrm{CH} 2 \mathrm{O}+\mathrm{O} 2<=>\mathrm{HCO}+\mathrm{HO} 2$ & & & $1.00 \mathrm{E}+14$ & 0.0 & 40000.0 \\
\hline 54. & $\mathrm{CH} 2 \mathrm{O}+\mathrm{HO} 2<=>\mathrm{HCO}+\mathrm{H} 2 \mathrm{O} 2$ & & & $1.00 \mathrm{E}+12$ & 0.0 & 8000.0 \\
\hline 55. & $\mathrm{CH} 3+\mathrm{O}<=>\mathrm{CH} 2 \mathrm{O}+\mathrm{H}$ & & & $8.43 E+13$ & 0.0 & 0.0 \\
\hline 56. & $\mathrm{CH} 3+\mathrm{OH}<=>\mathrm{CH} 2+\mathrm{H} 2 \mathrm{O}$ & & & $5.60 \mathrm{E}+07$ & 1.6 & 5420.0 \\
\hline 57. & $\mathrm{CH} 3+02<=>0+\mathrm{CH} 3 \mathrm{O}$ & & & $3.08 \mathrm{E}+13$ & 0.0 & 28800.0 \\
\hline 58. & $\mathrm{CH} 3+\mathrm{O} 2<=>\mathrm{OH}+\mathrm{CH} 2 \mathrm{O}$ & & & $3.60 \mathrm{E}+10$ & 0.0 & 8940.0 \\
\hline 59. & $\mathrm{CH} 3+\mathrm{HO} 2<=>\mathrm{CH} 3 \mathrm{O}+\mathrm{OH}$ & & & $1.34 \mathrm{E}+13$ & 0.0 & 0.0 \\
\hline 60. & $\mathrm{CH} 3+\mathrm{CH} 2<=>\mathrm{C} 2 \mathrm{H} 4+\mathrm{H}$ & & & $4.00 \mathrm{E}+13$ & 0.0 & 0.0 \\
\hline 61. & $2 \mathrm{CH} 3(+\mathrm{M})<=>\mathrm{C} 2 \mathrm{H} 6(+\mathrm{M})$ & & & $2.12 \mathrm{E}+16$ & -1.0 & 620.0 \\
\hline & Low pressure limit: & $0.17700 \mathrm{E}+51$ & $-0.96700 \mathrm{E}+01$ & $0.62200 \mathrm{E}+0$ & & \\
\hline & TROE centering: & $0.53250 \mathrm{E}+00$ & $0.15100 \mathrm{E}+03$ & $0.10380 \mathrm{E}+0$ & & $.49700 \mathrm{E}+04$ \\
\hline & $\mathrm{H} 2$ & Enhanced by & $2.000 \mathrm{E}+00$ & & & \\
\hline & $\mathrm{H} 2 \mathrm{O}$ & Enhanced by & $6.000 \mathrm{E}+00$ & & & \\
\hline & $\mathrm{CO}$ & Enhanced by & $1.500 \mathrm{E}+00$ & & & \\
\hline & $\mathrm{CO} 2$ & Enhanced by & $2.000 \mathrm{E}+00$ & & & \\
\hline
\end{tabular}


$\mathrm{C} 2 \mathrm{H} 6$

62. $2 \mathrm{CH} 3<=>\mathrm{H}+\mathrm{C} 2 \mathrm{H} 5$

63. $\mathrm{CH} 3+\mathrm{HCCO}<=>\mathrm{C} 2 \mathrm{H} 4+\mathrm{CO}$

64. $\mathrm{CH} 3 \mathrm{O}+\mathrm{H}<=>\mathrm{CH} 2 \mathrm{O}+\mathrm{H} 2$

65. $\mathrm{CH} 3 \mathrm{O}+\mathrm{H}<=>\mathrm{CH} 3+\mathrm{OH}$

66. $\mathrm{CH} 3 \mathrm{O}+\mathrm{O}<=>\mathrm{CH} 2 \mathrm{O}+\mathrm{OH}$

67. $\mathrm{CH} 3 \mathrm{O}+\mathrm{OH}<=>\mathrm{CH} 2 \mathrm{O}+\mathrm{H} 2 \mathrm{O}$

68. $\mathrm{CH} 3 \mathrm{O}+\mathrm{O} 2<=>\mathrm{CH} 2 \mathrm{O}+\mathrm{HO} 2$

69. $\mathrm{HCCO}+\mathrm{O}<=>\mathrm{H}+2 \mathrm{CO}$

70. $\mathrm{HCCO}+\mathrm{O} 2<=>\mathrm{OH}+2 \mathrm{CO}$

71. $\mathrm{HCCO}+\mathrm{CH} 2<=>\mathrm{C} 2 \mathrm{H} 3+\mathrm{CO}$

72. $2 \mathrm{HCCO}<=>\mathrm{C} 2 \mathrm{H} 2+2 \mathrm{CO}$

73. $\mathrm{C} 2 \mathrm{H} 2(+\mathrm{M})<=>\mathrm{H} 2 \mathrm{CC}(+\mathrm{M})$

Low pressure limit:

$\begin{array}{lll}\text { H2 } & \text { Enhanced by } & 2.000 \mathrm{E}+00 \\ \mathrm{H} 20 & \text { Enhanced by } & 6.000 \mathrm{E}+00 \\ \mathrm{CO} & \text { Enhanced by } & 1.500 \mathrm{E}+00 \\ \mathrm{C} 2 & \text { Enhanced by } & 2.000 \mathrm{E}+00 \\ \mathrm{C} 2 \mathrm{H} 4 & \text { Enhanced by } & 2.500 \mathrm{E}+00 \\ \mathrm{C} 2 \mathrm{H} 6 & \text { Enhanced by } & 3.000 \mathrm{E}+00\end{array}$

74. $\mathrm{C} 2 \mathrm{H} 3(+\mathrm{M})<=>\mathrm{C} 2 \mathrm{H} 2+\mathrm{H}(+\mathrm{M})$

Low pressure limit:

TROE centering:

$0.19820 \mathrm{E}+01$

$0.53840 \mathrm{E}+04$

H2

$\mathrm{H} 2 \mathrm{O}$

CO

$\mathrm{CO} 2$
$3.86 \mathrm{E}+08$

1.6

37048.2

$\begin{array}{lll}4.99 \mathrm{E}+12 & 0.1 & 10600.0\end{array}$

$\begin{array}{lll}5.00 \mathrm{E}+13 & 0.0 & 0.0\end{array}$

$\begin{array}{lll}2.00 \mathrm{E}+13 & 0.0 & 0.0\end{array}$

$\begin{array}{lll}3.20 \mathrm{E}+13 & 0.0 & 0.0\end{array}$

$\begin{array}{lll}1.00 \mathrm{E}+13 & 0.0 & 0.0\end{array}$

$\begin{array}{lll}4.28 \mathrm{E}-13 & 7.6 & -3530.0\end{array}$

$\begin{array}{lll}1.00 \mathrm{E}+14 & 0.0 & 0.0\end{array}$

$\begin{array}{lll}1.60 \mathrm{E}+12 & 0.0 & 854.0\end{array}$

$\begin{array}{lll}3.00 \mathrm{E}+13 & 0.0 & 0.0\end{array}$

$\begin{array}{lll}1.00 \mathrm{E}+13 & 0.0 & 0.0\end{array}$

$8.00 E+14 \quad-0.5 \quad 50750.0$

$0.49700 \mathrm{E}+05$

\section{$0.35800 \mathrm{E}+05$}

$0.42930 \mathrm{E}+01-0.79500 \mathrm{E}-01$ $\begin{array}{lll}5.00 \mathrm{E}+12 & 0.0 & 0.0\end{array}$ 


$\begin{array}{lll}\mathrm{C} 2 \mathrm{H} 2 & \text { Enhanced by } & 3.000 \mathrm{E}+00 \\ \mathrm{C} 2 \mathrm{H} 4 & \text { Enhanced by } & 3.000 \mathrm{E}+00 \\ \mathrm{C} 2 \mathrm{H} 6 & \text { Enhanced by } & 3.000 \mathrm{E}+00\end{array}$

75. $\mathrm{C} 2 \mathrm{H} 2+\mathrm{O}<=>\mathrm{CH} 2+\mathrm{CO}$

$4.08 \mathrm{E}+06 \quad 2.0 \quad 1900.0$

76. $\mathrm{C} 2 \mathrm{H} 2+\mathrm{O}<=>\mathrm{HCCO}+\mathrm{H}$

$\begin{array}{lll}1.63 \mathrm{E}+07 & 2.0 & 1900.0\end{array}$

77. $\mathrm{C} 2 \mathrm{H} 2+\mathrm{OH}<=>\mathrm{CH} 2 \mathrm{CO}+\mathrm{H}$

$2.18 \mathrm{E}-04 \quad 4.5 \quad-1000.0$

78. $\mathrm{C} 2 \mathrm{H} 2+\mathrm{OH}<=>\mathrm{CH} 3+\mathrm{CO}$

$\begin{array}{lll}4.83 \mathrm{E}-04 & 4.0 & -2000.0\end{array}$

79. $\mathrm{C} 2 \mathrm{H} 2+\mathrm{HCO}<=>\mathrm{C} 2 \mathrm{H} 3+\mathrm{CO}$

$1.00 \mathrm{E}+07 \quad 2.0 \quad 6000.0$

80. $\mathrm{H} 2 \mathrm{CC}+\mathrm{H}<=>\mathrm{C} 2 \mathrm{H} 2+\mathrm{H}$

$1.00 \mathrm{E}+14 \quad 0.0 \quad 0.0$

81. $\mathrm{H} 2 \mathrm{CC}+\mathrm{OH}<=>\mathrm{CH} 2 \mathrm{CO}+\mathrm{H}$

$\begin{array}{lll}2.00 \mathrm{E}+13 & 0.0 & 0.0\end{array}$

82. $\mathrm{H} 2 \mathrm{CC}+\mathrm{O} 2<=>2 \mathrm{HCO}$

$1.00 \mathrm{E}+13 \quad 0.0$

0.0

83. $\mathrm{CH} 2 \mathrm{CO}+\mathrm{H}(+\mathrm{M})<=>\mathrm{CH} 2 \mathrm{CHO}(+\mathrm{M})$

$\begin{array}{lll}3.30 E+14 & -0.1 & 8500.0\end{array}$

Low pressure limit: $\quad 0.38000 \mathrm{E}+42-0.76400 \mathrm{E}+01$

$0.11900 \mathrm{E}+05$

TROE centering:

$0.33700 \mathrm{E}+00 \quad 0.17070 \mathrm{E}+04$

$0.32000 \mathrm{E}+04 \quad 0.41310 \mathrm{E}+04$

$\mathrm{H} 2$

$\mathrm{H} 2 \mathrm{O}$

CO

$\mathrm{CO2}$

$\mathrm{C} 2 \mathrm{H} 2$

$\mathrm{C} 2 \mathrm{H} 4$

$\mathrm{C} 2 \mathrm{H} 6$
Enhanced by $2.000 \mathrm{E}+00$

Enhanced by $\quad 6.000 \mathrm{E}+00$

Enhanced by $1.500 \mathrm{E}+00$

Enhanced by $2.000 E+00$

Enhanced by

$3.000 \mathrm{E}+00$

Enhanced by

3. $000 \mathrm{E}+00$

Enhanced by

$3.000 \mathrm{E}+00$

84. $\mathrm{CH} 2 \mathrm{CO}+\mathrm{H}<=>\mathrm{HCCO}+\mathrm{H} 2$

$\begin{array}{lll}5.00 \mathrm{E}+13 & 0.0 & 8000.0\end{array}$

85. $\mathrm{CH} 2 \mathrm{CO}+\mathrm{H}<=>\mathrm{CH} 3+\mathrm{CO}$

$\begin{array}{lll}1.50 \mathrm{E}+09 & 1.4 & 2690.0\end{array}$

86. $\mathrm{CH} 2 \mathrm{CO}+\mathrm{O}<=>\mathrm{HCCO}+\mathrm{OH}$

$\begin{array}{lll}1.00 \mathrm{E}+13 & 0.0 & 8000.0\end{array}$

87. $\mathrm{CH} 2 \mathrm{CO}+\mathrm{O}<=>\mathrm{CH} 2+\mathrm{CO} 2$

$\begin{array}{lll}1.75 \mathrm{E}+12 & 0.0 & 1350.0\end{array}$

88. $\mathrm{CH} 2 \mathrm{CO}+\mathrm{OH}<=>\mathrm{HCCO}+\mathrm{H} 2 \mathrm{O}$

$\begin{array}{lll}7.50 \mathrm{E}+12 & 0.0 & 2000.0\end{array}$

89. $\mathrm{C} 2 \mathrm{H} 3+\mathrm{H}(+\mathrm{M})<=>\mathrm{C} 2 \mathrm{H} 4(+\mathrm{M})$

$6.08 \mathrm{E}+12 \quad 0.3 \quad 280.0$ 

Low pressure limit: $0.14000 \mathrm{E}+31-0.38600 \mathrm{E}+01$
$0.33200 \mathrm{E}+04$
TROE centering:
$0.78200 \mathrm{E}+00 \quad 0.20750 \mathrm{E}+03$
$0.26630 \mathrm{E}+04$
$0.60950 \mathrm{E}+04$

$\mathrm{H} 2$

Enhanced by $2.000 \mathrm{E}+00$

$\mathrm{H} 2 \mathrm{O}$

Enhanced by $\quad 6.000 \mathrm{E}+00$

CO

Enhanced by $1.500 \mathrm{E}+00$

$\mathrm{CO} 2$

Enhanced by $2.000 \mathrm{E}+00$

$\mathrm{C} 2 \mathrm{H} 2$

Enhanced by $3.000 \mathrm{E}+00$

$\mathrm{C} 2 \mathrm{H} 4$

Enhanced by $3.000 \mathrm{E}+00$

$\mathrm{C} 2 \mathrm{H} 6$

Enhanced by $3.000 \mathrm{E}+00$

90. $\mathrm{C} 2 \mathrm{H} 3+\mathrm{H}<=>\mathrm{C} 2 \mathrm{H} 2+\mathrm{H} 2$

$9.00 \mathrm{E}+13 \quad 0.0$

0.0

91. $\mathrm{C} 2 \mathrm{H} 3+\mathrm{H}<=>\mathrm{H} 2 \mathrm{CC}+\mathrm{H} 2$

$6.00 \mathrm{E}+13$

0.0

0.0

92. $\mathrm{C} 2 \mathrm{H} 3+\mathrm{O}<=>\mathrm{CH} 2 \mathrm{CO}+\mathrm{H}$

4. $80 \mathrm{E}+13$

0.0

0.0

93. $\mathrm{C} 2 \mathrm{H} 3+\mathrm{O}<=>\mathrm{CH} 3+\mathrm{CO}$

4.80E+13 0.0

0.0

94. $\mathrm{C} 2 \mathrm{H} 3+\mathrm{OH}<=>\mathrm{C} 2 \mathrm{H} 2+\mathrm{H} 2 \mathrm{O}$

$\begin{array}{lll}3.01 \mathrm{E}+13 & 0.0 & 0.0\end{array}$

95. $\mathrm{C} 2 \mathrm{H} 3+\mathrm{O} 2<=>\mathrm{C} 2 \mathrm{H} 2+\mathrm{HO} 2$

$\begin{array}{lll}1.34 \mathrm{E}+06 & 1.6 & -383.4\end{array}$

96. $\mathrm{C} 2 \mathrm{H} 3+\mathrm{O} 2<=>\mathrm{CH} 2 \mathrm{CHO}+\mathrm{O}$

$3.00 \mathrm{E}+11 \quad 0.3$

11.0

97. $\mathrm{C} 2 \mathrm{H} 3+\mathrm{O} 2<=>\mathrm{HCO}+\mathrm{CH} 2 \mathrm{O}$

$\begin{array}{lll}4.60 \mathrm{E}+16 & -1.4 \quad 1010.0\end{array}$

98. $\mathrm{C} 2 \mathrm{H} 3+\mathrm{HO} 2<=>\mathrm{CH} 2 \mathrm{CHO}+\mathrm{OH}$

$\begin{array}{lll}1.00 \mathrm{E}+13 & 0.0 & 0.0\end{array}$

99. $\mathrm{C} 2 \mathrm{H} 3+\mathrm{H} 2 \mathrm{O} 2<=>\mathrm{C} 2 \mathrm{H} 4+\mathrm{HO} 2$

$1.21 \mathrm{E}+10 \quad 0.0 \quad-596.0$

100. $\mathrm{C} 2 \mathrm{H} 3+\mathrm{HCO}<=>\mathrm{C} 2 \mathrm{H} 4+\mathrm{CO}$

$9.03 E+13 \quad 0.0$

0.0

101. $2 \mathrm{C} 2 \mathrm{H} 3<=>\mathrm{C} 2 \mathrm{H} 2+\mathrm{C} 2 \mathrm{H} 4$

$9.60 \mathrm{E}+11$

0.0

0.0

102. $\mathrm{CH} 2 \mathrm{CHO}<=>\mathrm{CH} 3+\mathrm{CO}$

$7.80 \mathrm{E}+41 \quad-9.1 \quad 46900.0$

103. $\mathrm{CH} 2 \mathrm{CHO}+\mathrm{H}<=>\mathrm{CH} 3+\mathrm{HCO}$

$9.00 \mathrm{E}+13 \quad 0.0$

0.0

104. $\mathrm{CH} 2 \mathrm{CHO}+\mathrm{H}<=>\mathrm{CH} 2 \mathrm{CO}+\mathrm{H} 2$

$2.00 \mathrm{E}+13 \quad 0.0 \quad 4000.0$

105. $\mathrm{CH} 2 \mathrm{CHO}+\mathrm{O}<=>\mathrm{CH} 2 \mathrm{CO}+\mathrm{OH}$

$2.00 \mathrm{E}+13 \quad 0.0 \quad 4000.0$

106. $\mathrm{CH} 2 \mathrm{CHO}+\mathrm{OH}<=>\mathrm{CH} 2 \mathrm{CO}+\mathrm{H} 2 \mathrm{O}$

$\begin{array}{lll}1.00 \mathrm{E}+13 & 0.0 & 2000.0\end{array}$

107. $\mathrm{CH} 2 \mathrm{CHO}+\mathrm{O} 2<=>\mathrm{CH} 2 \mathrm{CO}+\mathrm{HO} 2$

$\begin{array}{lll}1.40 \mathrm{E}+11 & 0.0 & 0.0\end{array}$ 


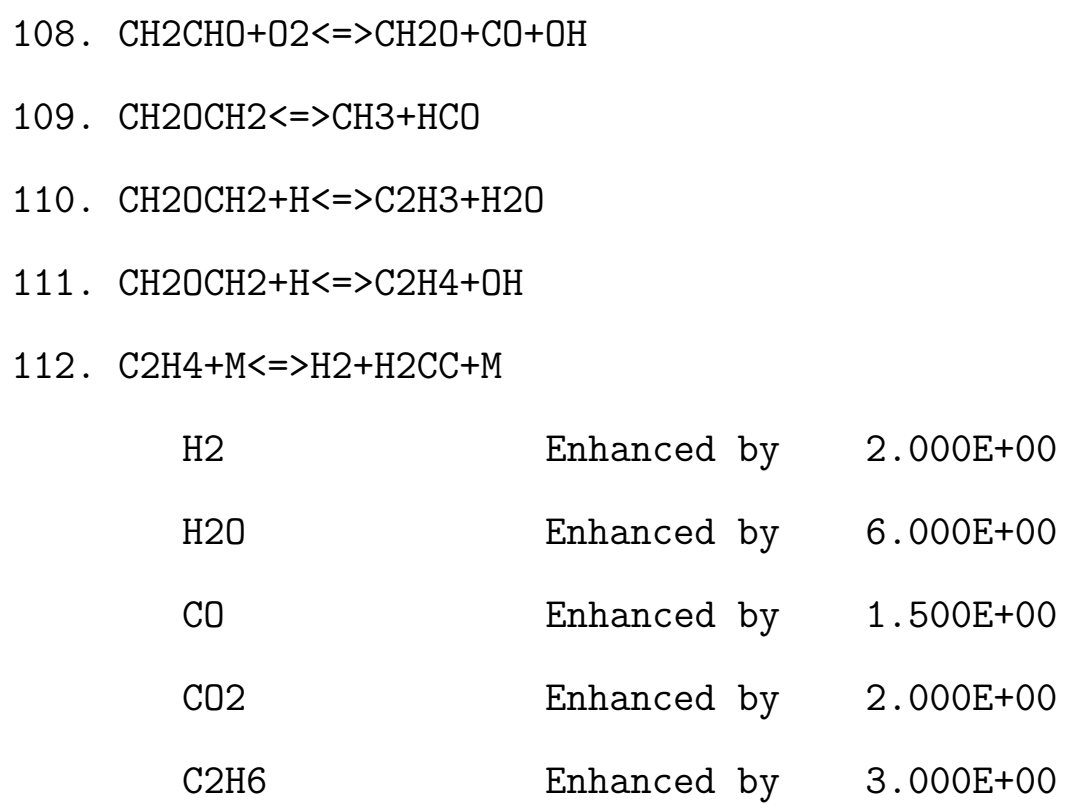

113. $\mathrm{C} 2 \mathrm{H} 4+\mathrm{H}(+\mathrm{M})<=>\mathrm{C} 2 \mathrm{H} 5(+\mathrm{M})$

$\begin{array}{lll}1.80 \mathrm{E}+10 & 0.0 & 0.0\end{array}$

3. $63 \mathrm{E}+13 \quad 0.0 \quad 57200.0$

$\begin{array}{lll}5.00 \mathrm{E}+09 & 0.0 & 5000.0\end{array}$

$9.51 \mathrm{E}+10 \quad 0.0 \quad 5000.0$

$\begin{array}{lll}7.86 \mathrm{E}+14 & 0.0 \quad 54245.0\end{array}$

Low pressure limit: $\quad 0.20270 \mathrm{E}+40-0.66420 \mathrm{E}+01$

$1.37 \mathrm{E}+09 \quad 1.5 \quad 1355.0$

$\begin{array}{clr}\text { TROE centering: } & -0.56900 \mathrm{E}+00 & 0.29900 \mathrm{E}+03 \\ \text { H2 } & \text { Enhanced by } & 2.000 \mathrm{E}+00 \\ \text { H2O } & \text { Enhanced by } & 6.000 \mathrm{E}+00 \\ \text { CO } & \text { Enhanced by } & 1.500 \mathrm{E}+00 \\ \text { CO2 } & \text { Enhanced by } & 2.000 \mathrm{E}+00 \\ \text { C2H6 } & \text { Enhanced by } & 3.000 \mathrm{E}+00\end{array}$

114. $\mathrm{C} 2 \mathrm{H} 4+\mathrm{H}<=>\mathrm{C} 2 \mathrm{H} 3+\mathrm{H} 2$

$5.07 \mathrm{E}+07 \quad 1.9 \quad 12950.0$

115. $\mathrm{C} 2 \mathrm{H} 4+\mathrm{O}<=>\mathrm{C} 2 \mathrm{H} 3+\mathrm{OH}$

$\begin{array}{lll}1.51 \mathrm{E}+07 & 1.9 & 3740.0\end{array}$

116. $\mathrm{C} 2 \mathrm{H} 4+\mathrm{O}<=>\mathrm{CH} 3+\mathrm{HCO}$

$\begin{array}{lll}1.92 \mathrm{E}+07 \quad 1.8 & 220.0\end{array}$

117. $\mathrm{C} 2 \mathrm{H} 4+\mathrm{O}<=>\mathrm{CH} 2+\mathrm{CH} 2 \mathrm{O}$

$3.84 \mathrm{E}+05 \quad 1.8 \quad 220.0$

118. $\mathrm{C} 2 \mathrm{H} 4+\mathrm{OH}<=>\mathrm{C} 2 \mathrm{H} 3+\mathrm{H} 2 \mathrm{O}$

$3.60 \mathrm{E}+06 \quad 2.0 \quad 2500.0$

119. $\mathrm{C} 2 \mathrm{H} 4+\mathrm{HCO}<=>\mathrm{C} 2 \mathrm{H} 5+\mathrm{CO}$

$1.00 \mathrm{E}+07 \quad 2.0 \quad 8000.0$

120. $\mathrm{C} 2 \mathrm{H} 4+\mathrm{O} 2<=>\mathrm{C} 2 \mathrm{H} 3+\mathrm{HO} 2$

$\begin{array}{lll}4.22 \mathrm{E}+13 & 0.0 & 60800.0\end{array}$

121. $\mathrm{C} 2 \mathrm{H} 4+\mathrm{HO} 2<=>\mathrm{CH} 2 \mathrm{OCH} 2+\mathrm{OH}$

$2.82 \mathrm{E}+12 \quad 0.0 \quad 17100.0$

122. $\mathrm{C} 2 \mathrm{H} 5+\mathrm{H}(+\mathrm{M})<=>\mathrm{C} 2 \mathrm{H} 6(+\mathrm{M})$

$5.21 \mathrm{E}+17-1.0 \quad 1580.0$ 

Low pressure limit:
$0.19900 \mathrm{E}+42-0.70800 \mathrm{E}+01$
$0.66850 \mathrm{E}+04$
TROE centering:
$0.84220 \mathrm{E}+00 \quad 0.12500 \mathrm{E}+03$
$0.22190 \mathrm{E}+04$
$0.68820 \mathrm{E}+04$

$\mathrm{H} 2$

Enhanced by $2.000 \mathrm{E}+00$

$\mathrm{H} 2 \mathrm{O}$

Enhanced by

$6.000 \mathrm{E}+00$

CO

Enhanced by

1. $500 \mathrm{E}+00$

$\mathrm{CO} 2$

Enhanced by

$2.000 \mathrm{E}+00$

$\mathrm{C} 2 \mathrm{H} 6$

Enhanced by

$3.000 E+00$

123. $\mathrm{C} 2 \mathrm{H} 5+\mathrm{H}<=>\mathrm{C} 2 \mathrm{H} 4+\mathrm{H} 2$

$2.00 \mathrm{E}+12 \quad 0.0$

0.0

124. $\mathrm{C} 2 \mathrm{H} 5+\mathrm{O}<=>\mathrm{CH} 3+\mathrm{CH} 2 \mathrm{O}$

1. $60 \mathrm{E}+13 \quad 0.0$

0.0

125. $\mathrm{C} 2 \mathrm{H} 5+\mathrm{O} 2<=>\mathrm{C} 2 \mathrm{H} 4+\mathrm{HO} 2$

$2.00 \mathrm{E}+10$

0.0

0.0

126. $\mathrm{C} 2 \mathrm{H} 5+\mathrm{HO} 2<=>\mathrm{C} 2 \mathrm{H} 6+\mathrm{O} 2$

3. $00 \mathrm{E}+11 \quad 0.0$

0.0

127. $\mathrm{C} 2 \mathrm{H} 5+\mathrm{HO} 2<=>\mathrm{C} 2 \mathrm{H} 4+\mathrm{H} 2 \mathrm{O} 2$

$3.00 \mathrm{E}+11 \quad 0.0$

0.0

128. $\mathrm{C} 2 \mathrm{H} 5+\mathrm{HO} 2<=>\mathrm{CH} 3+\mathrm{CH} 2 \mathrm{O}+\mathrm{OH}$

$2.40 \mathrm{E}+13 \quad 0.0$

0.0

129. $\mathrm{C} 2 \mathrm{H} 5+\mathrm{H} 2 \mathrm{O} 2<=>\mathrm{C} 2 \mathrm{H} 6+\mathrm{HO} 2$

$8.70 \mathrm{E}+09$

0.0

974.0

130. $\mathrm{C} 2 \mathrm{H} 6+\mathrm{H}<=>\mathrm{C} 2 \mathrm{H} 5+\mathrm{H} 2$

$\begin{array}{lll}1.15 \mathrm{E}+08 & 1.9 & 7530.0\end{array}$

131. $\mathrm{C} 2 \mathrm{H} 6+\mathrm{O}<=>\mathrm{C} 2 \mathrm{H} 5+\mathrm{OH}$

8. $98 \mathrm{E}+07$

1.9

5690.0

132. $\mathrm{C} 2 \mathrm{H} 6+\mathrm{OH}<=>\mathrm{C} 2 \mathrm{H} 5+\mathrm{H} 2 \mathrm{O}$

$\begin{array}{lll}3.54 \mathrm{E}+06 & 2.1 & 870.0\end{array}$

NOTE: A units mole-cm-sec-K, E units cal/mole 


\section{Appendix B}

\section{Reduced n-Dodecane Pyrolysis Reaction Mechanism}

The skeletal reduced reaction model for n-dodecane pyrolysis developed in this study and used for the two-dimensional axi-symmetric tube reactor simulation consists of 50 species and 190 reactions. The units for this mechanism are in moles, centimeters, seconds, Kelvin, and calories. The units of activation energy (E) are in calories per mole. The units of pre-exponential factor (A) for each reaction can be determined by the order of the reaction and requires dimensional analysis. 


$\begin{array}{ll}\text { ELEMENTS } & \text { ATOMIC } \\ \text { CONSIDERED } & \text { WEIGHT } \\ & \\ \text { 1. } \mathrm{O} & 15.9994 \\ 2 . \mathrm{H} & 1.00797 \\ 3 . \mathrm{C} & 12.0112 \\ 4 . \mathrm{N} & 14.0067 \\ - & \end{array}$

\section{C}

P H

H A

A $R$

SPECIES

S G MOLECULAR TEMPERATURE ELEMENT COUNT

CONSIDERED

E E WEIGHT LOW HIGH $\mathrm{O} \quad \mathrm{H} \quad \mathrm{C} \quad \mathrm{N}$

\begin{tabular}{|c|c|c|c|c|c|c|c|}
\hline 1. $\mathrm{H}$ & G & 1.00797 & 200.0 & 3500.0 & 0 & 1 & 0 \\
\hline 2. $\mathrm{H} 2$ & G & 2.01594 & 200.0 & 3500.0 & 0 & 2 & 0 \\
\hline 3. $\mathrm{CH} 3$ & G & 15.03506 & 200.0 & 3500.0 & 0 & 3 & 1 \\
\hline 4. $\mathrm{CH} 4$ & G & 16.04303 & 200.0 & 3500.0 & 0 & 4 & 1 \\
\hline 5. $\mathrm{C} 2 \mathrm{H} 2$ & G & 26.03824 & 200.0 & 3500.0 & 0 & 2 & 2 \\
\hline 6. $\mathrm{H} 2 \mathrm{CC}$ & G & 26.03824 & 200.0 & 6000.0 & 0 & 2 & 2 \\
\hline 7. $\mathrm{C} 2 \mathrm{H} 3$ & G & 27.04621 & 200.0 & 3500.0 & 0 & 3 & 2 \\
\hline 8. $\mathrm{C} 2 \mathrm{H} 4$ & G & 28.05418 & 200.0 & 3500.0 & 0 & 4 & 2 \\
\hline 9. $\mathrm{C} 2 \mathrm{H} 5$ & G & 29.06215 & 200.0 & 3500.0 & 0 & 5 & 2 \\
\hline o. $\mathrm{C} 2 \mathrm{H} 6$ & G & 30.07012 & 200.0 & 3500.0 & 0 & 6 & 2 \\
\hline
\end{tabular}




\begin{tabular}{|c|c|c|c|c|c|c|c|}
\hline 11. $\mathrm{pC} 3 \mathrm{H} 4$ & G 0 & 40.06533 & 200.0 & 6000.0 & 0 & 4 & 3 \\
\hline 12. aC3H4 & G 0 & 40.06533 & 200.0 & 6000.0 & 0 & 4 & 3 \\
\hline 13. aC3H5 & G 0 & 41.07330 & 300.0 & 3000.0 & 0 & 5 & 3 \\
\hline 14. С3H6 & G 0 & 42.08127 & 300.0 & 5000.0 & 0 & 6 & 3 \\
\hline 5. $\mathrm{nC} 3 \mathrm{H} 7$ & G 0 & 43.08924 & 300.0 & 3000.0 & 0 & 7 & 3 \\
\hline 16. С3H8 & G 0 & 44.09721 & 300.0 & 3000.0 & 0 & 8 & 3 \\
\hline 17. C4H81 & G 0 & 56.10836 & 300.0 & 5000.0 & 0 & 8 & 4 \\
\hline 18. $\mathrm{pC} 4 \mathrm{H} 9$ & G 0 & 57.11633 & 300.0 & 5000.0 & 0 & 9 & 4 \\
\hline 19. $\mathrm{NC} 12 \mathrm{H} 26$ & G 0 & 170.34102 & 300.0 & 5000.0 & 0 & 26 & 12 \\
\hline 20. $\mathrm{PXC} 12 \mathrm{H} 25$ & G 0 & 169.33305 & 300.0 & 5000.0 & 0 & 25 & 12 \\
\hline 21. $\mathrm{SXC} 12 \mathrm{H} 25$ & G 0 & 169.33305 & 300.0 & 5000.0 & 0 & 25 & 12 \\
\hline 22. PXC11H23 & G 0 & 155.30596 & 300.0 & 5000.0 & 0 & 23 & 11 \\
\hline 23. $\mathrm{C} 11 \mathrm{H} 22$ & G 0 & 154.29799 & 300.0 & 5000.0 & 0 & 22 & 11 \\
\hline 24. $\mathrm{PXC} 10 \mathrm{H} 21$ & G 0 & 141.27887 & 300.0 & 5000.0 & 0 & 21 & 10 \\
\hline 25. $\mathrm{C} 10 \mathrm{H} 2 \mathrm{O}$ & G 0 & 140.27090 & 300.0 & 5000.0 & 0 & 20 & 10 \\
\hline 26. РXC9H19 & G 0 & 127.25178 & 300.0 & 5000.0 & 0 & 19 & 9 \\
\hline 27. $\mathrm{C} 9 \mathrm{H} 18$ & G 0 & 126.24381 & 300.0 & 5000.0 & 0 & 18 & 9 \\
\hline 28. PXC8H17 & G 0 & 113.22469 & 300.0 & 5000.0 & 0 & 17 & 8 \\
\hline 29. $\mathrm{C} 8 \mathrm{H} 16$ & G 0 & 112.21672 & 300.0 & 5000.0 & 0 & 16 & 8 \\
\hline 30. PXC7H15 & G 0 & 99.19760 & 300.0 & 5000.0 & 0 & 15 & 7 \\
\hline 31. SXC7H15 & G 0 & 99.19760 & 300.0 & 5000.0 & 0 & 15 & 7 \\
\hline 32. $\mathrm{C} 7 \mathrm{H} 14$ & G 0 & 98.18963 & 300.0 & 5000.0 & 0 & 14 & 7 \\
\hline 33. PXC6H13 & G 0 & 85.17051 & 300.0 & 5000.0 & 0 & 13 & 6 \\
\hline 34. SXC6H13 & G 0 & 85.17051 & 300.0 & 5000.0 & 0 & 13 & 6 \\
\hline 35. $\mathrm{C} 6 \mathrm{H} 12$ & G 0 & 84.16254 & 300.0 & 5000.0 & 0 & 12 & 6 \\
\hline 36. PXC5H11 & G 0 & 71.14342 & 300.0 & 5000.0 & 0 & 11 & 5 \\
\hline 37. SXC5H11 & G 0 & 71.14342 & 300.0 & 5000.0 & 0 & 11 & 5 \\
\hline
\end{tabular}




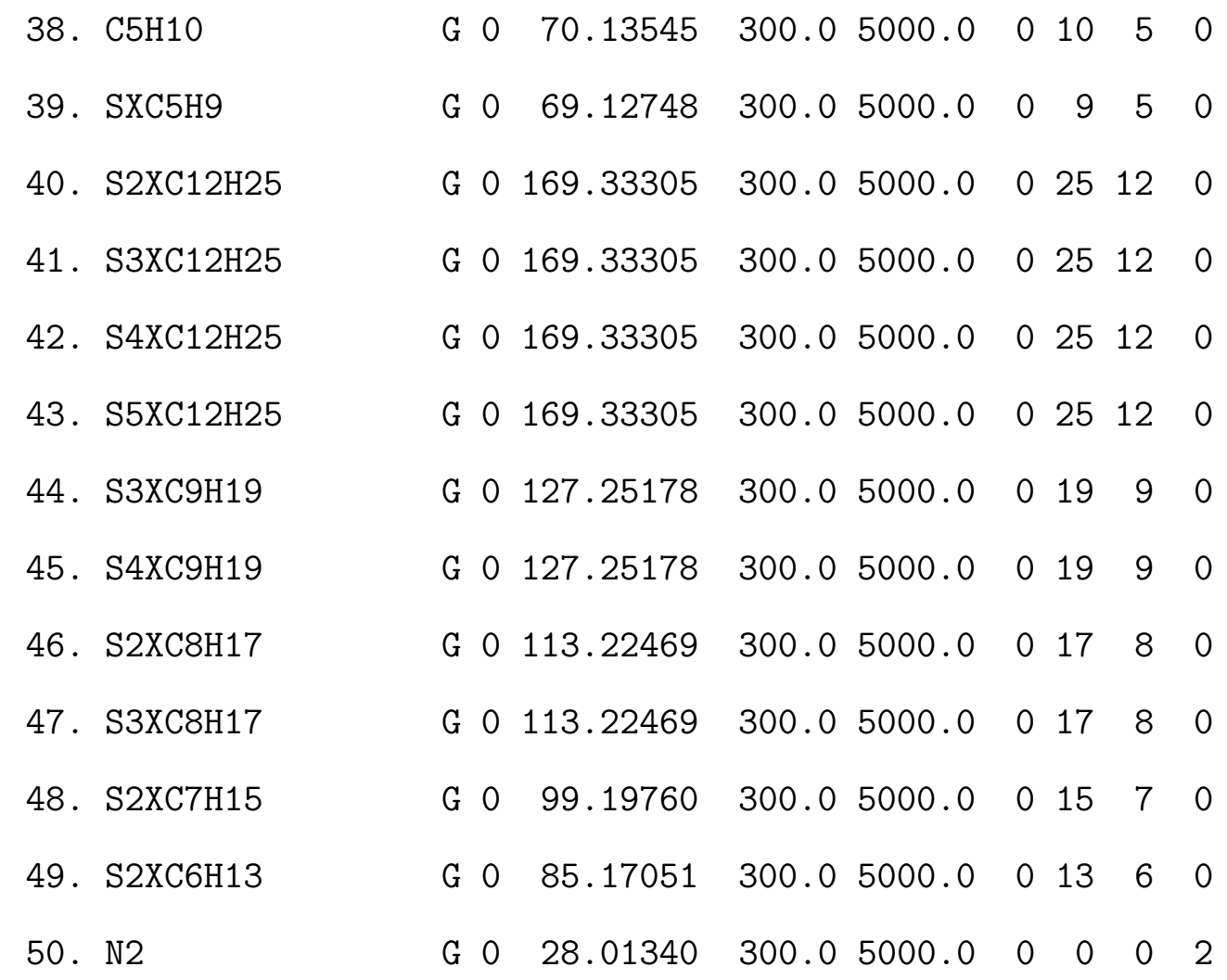

$(\mathrm{k}=\mathrm{A} T * * \mathrm{~b} \exp (-\mathrm{E} / \mathrm{RT}))$

REACTIONS CONSIDERED

A $\quad$ b $\quad$ E

1. $2 \mathrm{H}+\mathrm{M}<=>\mathrm{H} 2+\mathrm{M}$

$1.00 \mathrm{E}+18-1.0$

0.0

H2 Enhanced by $0.000 \mathrm{E}+00$

2. $2 \mathrm{H}+\mathrm{H} 2<=>2 \mathrm{H} 2$

$9.00 \mathrm{E}+16 \quad-0.6 \quad 0.0$

3. $\mathrm{CH} 3+\mathrm{H}(+\mathrm{M})<=>\mathrm{CH} 4(+\mathrm{M})$

$1.01 \mathrm{E}+16 \quad-0.6 \quad 383.0$

Low pressure limit:

$0.19720 \mathrm{E}+34-0.47600 \mathrm{E}+01$

$0.24400 \mathrm{E}+04$

TROE centering:

$0.78300 \mathrm{E}+00$

$0.74000 \mathrm{E}+02$

$0.29410 \mathrm{E}+04$

$0.69640 \mathrm{E}+04$

$\mathrm{H} 2$

Enhanced by $2.000 \mathrm{E}+00$ 


$\begin{array}{lll}\text { CH4 } & \text { Enhanced by } & 2.000 \mathrm{E}+00 \\ \mathrm{C} 2 \mathrm{H} 6 & \text { Enhanced by } & 3.000 \mathrm{E}+00\end{array}$

4. $2 \mathrm{CH} 3(+\mathrm{M})<=>\mathrm{C} 2 \mathrm{H} 6(+\mathrm{M})$

$2.44 \mathrm{E}+16 \quad-1.0 \quad 620.0$

Low pressure limit:

$0.20340 \mathrm{E}+51-0.96700 \mathrm{E}+01 \quad 0.62200 \mathrm{E}+04$

TROE centering:

$0.53250 \mathrm{E}+00 \quad 0.15100 \mathrm{E}+03$

$0.10380 \mathrm{E}+04 \quad 0.49700 \mathrm{E}+04$

H2

Enhanced by $2.000 \mathrm{E}+00$

$\mathrm{CH} 4$

Enhanced by

$2.000 \mathrm{E}+00$

$\mathrm{C} 2 \mathrm{H} 6$

Enhanced by $3.000 \mathrm{E}+00$

5. $2 \mathrm{CH} 3<=>\mathrm{H}+\mathrm{C} 2 \mathrm{H} 5$

$5.58 \mathrm{E}+12 \quad 0.1 \quad 10600.0$

6. $\mathrm{CH} 4+\mathrm{H}<=>\mathrm{CH} 3+\mathrm{H} 2$

$\begin{array}{lll}6.60 \mathrm{E}+08 & 1.6 & 10840.0\end{array}$

7. $\mathrm{C} 2 \mathrm{H} 2(+\mathrm{M})<=>\mathrm{H} 2 \mathrm{CC}(+\mathrm{M})$

$8.00 \mathrm{E}+14 \quad-0.5 \quad 50750.0$

Low pressure limit:

$0.24500 \mathrm{E}+16-0.64000 \mathrm{E}+00$

$0.49700 \mathrm{E}+05$

$\begin{array}{lll}\mathrm{H} 2 & \text { Enhanced by } & 2.000 \mathrm{E}+00 \\ \mathrm{CH} 4 & \text { Enhanced by } & 2.000 \mathrm{E}+00 \\ \mathrm{C} 2 \mathrm{H} 4 & \text { Enhanced by } & 2.500 \mathrm{E}+00 \\ \mathrm{C} 2 \mathrm{H} 6 & \text { Enhanced by } & 3.000 \mathrm{E}+00\end{array}$

8. $\mathrm{C} 2 \mathrm{H} 3(+\mathrm{M})<=>\mathrm{C} 2 \mathrm{H} 2+\mathrm{H}(+\mathrm{M})$

$\begin{array}{lll}3.63 \mathrm{E}+08 & 1.6 \quad 37048.2\end{array}$

Low pressure limit: $0.24140 \mathrm{E}+28-0.34000 \mathrm{E}+01$

$0.35800 \mathrm{E}+05$

TROE centering:

$0.19820 \mathrm{E}+01 \quad 0.53840 \mathrm{E}+04$

$0.42900 \mathrm{E}+01-0.80000 \mathrm{E}-01$

H2

Enhanced by $2.000 \mathrm{E}+00$

$\mathrm{CH} 4$

Enhanced by $2.000 \mathrm{E}+00$

$\mathrm{C} 2 \mathrm{H} 2$

Enhanced by $3.000 \mathrm{E}+00$

C2H4

Enhanced by

$3.000 E+00$

$\mathrm{C} 2 \mathrm{H} 6$

Enhanced by

$3.000 \mathrm{E}+00$

9. $\mathrm{C} 2 \mathrm{H} 2+\mathrm{CH} 3<=>\mathrm{pC} 3 \mathrm{H} 4+\mathrm{H}$

$2.42 \mathrm{E}+09 \quad 1.1 \quad 13644.0$

10. $\mathrm{C} 2 \mathrm{H} 2+\mathrm{CH} 3<=>\mathrm{aC} 3 \mathrm{H} 4+\mathrm{H}$

$\begin{array}{lll}5.14 \mathrm{E}+09 & 0.9 & 22153.0\end{array}$

11. $\mathrm{C} 2 \mathrm{H} 2+\mathrm{CH} 3<=>\mathrm{aC} 3 \mathrm{H} 5$

$2.68 \mathrm{E}+53-12.8 \quad 35730.0$ 
12. $\mathrm{H} 2 \mathrm{CC}+\mathrm{H}<=>\mathrm{C} 2 \mathrm{H} 2+\mathrm{H}$

13. $\mathrm{C} 2 \mathrm{H} 3+\mathrm{H}(+\mathrm{M})<=>\mathrm{C} 2 \mathrm{H} 4(+\mathrm{M})$
Low pressure limit:

TROE centering:

$\mathrm{H} 2$

$\mathrm{CH} 4$

$\mathrm{C} 2 \mathrm{H} 2$

$\mathrm{C} 2 \mathrm{H} 4$

$\mathrm{C} 2 \mathrm{H} 6$
$0.14000 \mathrm{E}+31-0.38600 \mathrm{E}+01$

$0.78200 \mathrm{E}+00$

Enhanced by

Enhanced by

Enhanced by

Enhanced by

Enhanced by
$2.000 E+00$

$2.000 \mathrm{E}+00$

$3.000 \mathrm{E}+00$

$3.000 \mathrm{E}+00$

$3.000 \mathrm{E}+00$
$1.00 \mathrm{E}+14 \quad 0.0$

$6.08 \mathrm{E}+12 \quad 0.3$

$0.33200 \mathrm{E}+04$

$0.26630 \mathrm{E}+04$

$0.60950 \mathrm{E}+04$

14. $\mathrm{C} 2 \mathrm{H} 3+\mathrm{H}<=>\mathrm{C} 2 \mathrm{H} 2+\mathrm{H} 2$

8. $80 \mathrm{E}+13$

0.0

0.0

15. $\mathrm{C} 2 \mathrm{H} 3+\mathrm{H}<=>\mathrm{H} 2 \mathrm{CC}+\mathrm{H} 2$

6. $40 \mathrm{E}+13$

0.0

0.0

16. $\mathrm{C} 2 \mathrm{H} 3+\mathrm{CH} 3<=>\mathrm{C} 2 \mathrm{H} 2+\mathrm{CH} 4$

$\begin{array}{lll}3.92 \mathrm{E}+11 & 0.0 & 0.0\end{array}$

17. $\mathrm{C} 2 \mathrm{H} 3+\mathrm{CH} 3(+\mathrm{M})<=>\mathrm{C} 3 \mathrm{H} 6(+\mathrm{M})$

$2.50 \mathrm{E}+13 \quad 0.0 \quad 0.0$

Low pressure limit: $0.42700 \mathrm{E}+59-0.11940 \mathrm{E}+02$

$0.97700 \mathrm{E}+04$

TROE centering:

$0.17500 \mathrm{E}+00$

$0.13410 \mathrm{E}+04$

$0.60000 \mathrm{E}+05$

$0.10140 \mathrm{E}+05$

$\mathrm{H} 2$

Enhanced by $2.000 \mathrm{E}+00$

CH4

Enhanced by

$2.000 \mathrm{E}+00$

$\mathrm{C} 2 \mathrm{H} 4$

Enhanced by

$3.000 \mathrm{E}+00$

$\mathrm{C} 2 \mathrm{H} 6$

Enhanced by

$3.000 E+00$

18. $\mathrm{C} 2 \mathrm{H} 3+\mathrm{CH} 3<=>\mathrm{aC} 3 \mathrm{H} 5+\mathrm{H}$

19. $2 \mathrm{C} 2 \mathrm{H} 3<=>\mathrm{C} 2 \mathrm{H} 2+\mathrm{C} 2 \mathrm{H} 4$

20. $\mathrm{C} 2 \mathrm{H} 4+\mathrm{M}<=>\mathrm{H} 2+\mathrm{H} 2 \mathrm{CC}+\mathrm{M}$

$\mathrm{H} 2$

Enhanced by

$2.000 \mathrm{E}+00$

$\mathrm{CH} 4$

Enhanced by

$2.000 \mathrm{E}+00$

$\mathrm{C} 2 \mathrm{H} 6$

Enhanced by

$3.000 \mathrm{E}+00$

21. $\mathrm{C} 2 \mathrm{H} 4+\mathrm{H}(+\mathrm{M})<=>\mathrm{C} 2 \mathrm{H} 5(+\mathrm{M})$

1. $24 \mathrm{E}+09 \quad 1.5 \quad 1355.0$ Low pressure limit:

$0.18360 \mathrm{E}+40-0.66420 \mathrm{E}+01$

$0.57690 \mathrm{E}+04$ 


$\begin{array}{lll}\text { TROE centering: } & -0.56900 \mathrm{E}+00 & 0.29900 \mathrm{E}+03 \\ \text { H2 } & \text { Enhanced by } & 2.000 \mathrm{E}+00 \\ \mathrm{CH} 4 & \text { Enhanced by } & 2.000 \mathrm{E}+00 \\ \mathrm{C} 2 \mathrm{H} 6 & \text { Enhanced by } & 3.000 \mathrm{E}+00\end{array}$

22. $\mathrm{C} 2 \mathrm{H} 4+\mathrm{H}<=>\mathrm{C} 2 \mathrm{H} 3+\mathrm{H} 2$

$5.00 \mathrm{E}+07 \quad 1.9 \quad 12950.0$

23. $\mathrm{C} 2 \mathrm{H} 4+\mathrm{CH} 3<=>\mathrm{C} 2 \mathrm{H} 3+\mathrm{CH} 4$

$\begin{array}{lll}4.46 \mathrm{E}+05 & 2.0 & 9200.0\end{array}$

24. $\mathrm{C} 2 \mathrm{H} 4+\mathrm{CH} 3<=>\mathrm{nC} 3 \mathrm{H} 7$

$\begin{array}{lll}3.30 \mathrm{E}+11 & 0.0 & 7700.0\end{array}$

25. $\mathrm{C} 2 \mathrm{H} 5+\mathrm{H}(+\mathrm{M})<=>\mathrm{C} 2 \mathrm{H} 6(+\mathrm{M})$

$5.21 \mathrm{E}+17 \quad-1.0 \quad 1580.0$

$\begin{array}{ccc}\text { Low pressure limit: } & 0.19900 \mathrm{E}+42 & -0.70800 \mathrm{E}+01 \\ \text { TROE centering: } & 0.84220 \mathrm{E}+00 & 0.12500 \mathrm{E}+03 \\ \text { H2 } & \text { Enhanced by } & 2.000 \mathrm{E}+00 \\ \text { CH4 } & \text { Enhanced by } & 2.000 \mathrm{E}+00 \\ \mathrm{C} 2 \mathrm{H} 6 & \text { Enhanced by } & 3.000 \mathrm{E}+00\end{array}$

26. $\mathrm{C} 2 \mathrm{H} 5+\mathrm{H}<=>\mathrm{C} 2 \mathrm{H} 4+\mathrm{H} 2$

$2.00 \mathrm{E}+12 \quad 0.0$

0.0

27. $\mathrm{C} 2 \mathrm{H} 5+\mathrm{CH} 3(+\mathrm{M})<=>\mathrm{C} 3 \mathrm{H} 8(+\mathrm{M})$

$\begin{array}{lll}4.90 \mathrm{E}+14 & -0.5 & 0.0\end{array}$

Low pressure limit:

$0.68000 \mathrm{E}+62-0.13420 \mathrm{E}+02$

$0.60000 \mathrm{E}+04$

TROE centering:

$0.10000 \mathrm{E}+01$

$0.10000 \mathrm{E}+04$

$0.14340 \mathrm{E}+04$

$0.53290 \mathrm{E}+04$

$\begin{array}{lll}\mathrm{H} 2 & \text { Enhanced by } & 2.000 \mathrm{E}+00 \\ \mathrm{CH} 4 & \text { Enhanced by } & 2.000 \mathrm{E}+00 \\ \mathrm{C} 2 \mathrm{H} 6 & \text { Enhanced by } & 3.000 \mathrm{E}+00\end{array}$

28. $\mathrm{C} 2 \mathrm{H} 5+\mathrm{C} 2 \mathrm{H} 3(+\mathrm{M})<=>\mathrm{C} 4 \mathrm{H} 81(+\mathrm{M})$

$1.50 \mathrm{E}+13 \quad 0.0$

0.0

Low pressure limit:

$0.15500 \mathrm{E}+57-0.11790 \mathrm{E}+02$

$0.89850 \mathrm{E}+04$

TROE centering:

$0.19800 \mathrm{E}+00$

$0.22780 \mathrm{E}+04$

$0.60000 \mathrm{E}+05$

$0.57230 \mathrm{E}+04$

$\begin{array}{lll}\mathrm{H} 2 & \text { Enhanced by } & 2.000 \mathrm{E}+00 \\ \mathrm{CH} 4 & \text { Enhanced by } & 2.000 \mathrm{E}+00 \\ \mathrm{C} 2 \mathrm{H} 6 & \text { Enhanced by } & 3.000 \mathrm{E}+00\end{array}$

29. $\mathrm{C} 2 \mathrm{H} 5+\mathrm{C} 2 \mathrm{H} 3<=>\mathrm{aC} 3 \mathrm{H} 5+\mathrm{CH} 3$

$\begin{array}{lll}3.90 \mathrm{E}+32 & -5.2 & 19747.0\end{array}$ 
30. $\mathrm{C} 2 \mathrm{H} 6+\mathrm{H}<=>\mathrm{C} 2 \mathrm{H} 5+\mathrm{H} 2$

31. $\mathrm{C} 2 \mathrm{H} 6+\mathrm{CH} 3<=>\mathrm{C} 2 \mathrm{H} 5+\mathrm{CH} 4$

32. $\mathrm{aC} 3 \mathrm{H} 4+\mathrm{H}<=>\mathrm{aC} 3 \mathrm{H} 5$

33. $\mathrm{pC} 3 \mathrm{H} 4<=>\mathrm{aC} 3 \mathrm{H} 4$

34. $\mathrm{pC} 3 \mathrm{H} 4+\mathrm{H}<=>\mathrm{aC} 3 \mathrm{H} 4+\mathrm{H}$

35. $\mathrm{pC} 3 \mathrm{H} 4+\mathrm{H}<=>\mathrm{aC} 3 \mathrm{H} 5$

36. $\mathrm{aC} 3 \mathrm{H} 5+\mathrm{H}(+\mathrm{M})<=>\mathrm{C} 3 \mathrm{H} 6(+\mathrm{M})$

Low pressure limit: $0.13010 \mathrm{E}+61-0.12000 \mathrm{E}+02$ TROE centering:

$\mathrm{H} 2$

CH4

$\mathrm{C} 2 \mathrm{H} 6$
$0.20000 \mathrm{E}-01$

Enhanced by

Enhanced by

Enhanced by
1. $15 \mathrm{E}+08$

$\begin{array}{lll}6.14 \mathrm{E}+06 & 1.7 & 10450.0\end{array}$

$1.62 \mathrm{E}+59-13.5 \quad 26949.0$

$5.15 \mathrm{E}+60 \quad-13.9 \quad 91117.0$

$6.27 \mathrm{E}+17 \quad-0.9 \quad 10079.0$

$4.91 \mathrm{E}+60 \quad-14.4 \quad 31644.0$

$\begin{array}{lll}1.96 \mathrm{E}+14 & 0.0 & 0.0\end{array}$

$0.59680 \mathrm{E}+04$

$0.10970 E+04 \quad 0.68600 E+04$

$0.10970 \mathrm{E}+04$

$2.000 \mathrm{E}+00$

$2.000 \mathrm{E}+00$

$3.000 \mathrm{E}+00$

37. $\mathrm{aC} 3 \mathrm{H} 5+\mathrm{H}<=>\mathrm{aC} 3 \mathrm{H} 4+\mathrm{H} 2$

$\begin{array}{lll}1.80 \mathrm{E}+13 & 0.0 & 0.0\end{array}$

38. $\mathrm{aC} 3 \mathrm{H} 5+\mathrm{CH} 3(+\mathrm{M})<=>\mathrm{C} 4 \mathrm{H} 81(+\mathrm{M})$

$9.92 \mathrm{E}+13 \quad-0.3 \quad-262.3$

Low pressure limit:

$0.38790 \mathrm{E}+61-0.12810 \mathrm{E}+02$

$0.62500 \mathrm{E}+04$

TROE centering:

$0.10400 \mathrm{E}+00$

$0.16060 \mathrm{E}+04$

$0.60000 \mathrm{E}+05$

$0.61180 \mathrm{E}+04$

$\mathrm{H} 2$

Enhanced by

$2.000 E+00$

$\mathrm{CH} 4$

Enhanced by

$2.000 \mathrm{E}+00$

$\mathrm{C} 2 \mathrm{H} 6$

Enhanced by

$3.000 E+00$

39. $\mathrm{aC} 3 \mathrm{H} 5+\mathrm{CH} 3<=>\mathrm{aC} 3 \mathrm{H} 4+\mathrm{CH} 4$

$\begin{array}{lll}3.00 \mathrm{E}+12 & -0.3 & -131.0\end{array}$

40. $\mathrm{C} 3 \mathrm{H} 6+\mathrm{H}(+\mathrm{M})<=>$ nC $3 \mathrm{H} 7(+\mathrm{M})$

1. $33 E+13$

0.0

3260.7

Low pressure limit:

$0.62600 \mathrm{E}+39-0.66600 \mathrm{E}+01$

$0.70000 \mathrm{E}+04$

TROE centering:

$0.10000 \mathrm{E}+01$

$0.10000 \mathrm{E}+04$

$0.13100 \mathrm{E}+04$

$0.48100 \mathrm{E}+05$

$\begin{array}{lll}\mathrm{H} 2 & \text { Enhanced by } & 2.000 \mathrm{E}+00 \\ \mathrm{CH} 4 & \text { Enhanced by } & 2.000 \mathrm{E}+00 \\ \mathrm{C} 2 \mathrm{H} 6 & \text { Enhanced by } & 3.000 \mathrm{E}+00\end{array}$

41. $\mathrm{C} 3 \mathrm{H} 6+\mathrm{H}<=>\mathrm{C} 2 \mathrm{H} 4+\mathrm{CH} 3$

$\begin{array}{lll}7.78 \mathrm{E}+21 & -2.4 & 11180.0\end{array}$ 
42. $\mathrm{C} 3 \mathrm{H} 6+\mathrm{H}<=>\mathrm{aC} 3 \mathrm{H} 5+\mathrm{H} 2$

43. $\mathrm{C} 3 \mathrm{H} 6+\mathrm{CH} 3<=>\mathrm{aC} 3 \mathrm{H} 5+\mathrm{CH} 4$

44. $\mathrm{nC} 3 \mathrm{H} 7+\mathrm{H}(+\mathrm{M})<=>\mathrm{C} 3 \mathrm{H} 8(+\mathrm{M})$

Low pressure limit:

TROE centering:

$\mathrm{H} 2$

$\mathrm{CH} 4$

$\mathrm{C} 2 \mathrm{H} 6$
$0.30100 E+49-0.93200 E+01$

$0.49800 \mathrm{E}+00$

$0.13140 \mathrm{E}+04$

Enhanced by

Enhanced by

Enhanced by
1. $29 \mathrm{E}+05$

2.5

$2.20 \mathrm{E}+00$

3.5

$3.60 \mathrm{E}+13$

0.0

$0.58340 \mathrm{E}+04$

$0.13140 \mathrm{E}+04$

$0.50000 \mathrm{E}+05$
45. $\mathrm{nC} 3 \mathrm{H} 7+\mathrm{H}<=>\mathrm{C} 2 \mathrm{H} 5+\mathrm{CH} 3$

46. $\mathrm{nC} 3 \mathrm{H} 7+\mathrm{H}<=>\mathrm{C} 3 \mathrm{H} 6+\mathrm{H} 2$

47. $\mathrm{nC} 3 \mathrm{H} 7+\mathrm{CH} 3<=>\mathrm{CH} 4+\mathrm{C} 3 \mathrm{H} 6$

48. $\mathrm{C} 3 \mathrm{H} 8+\mathrm{H}<=>\mathrm{H} 2+\mathrm{nC} 3 \mathrm{H} 7$

49. $\mathrm{C} 3 \mathrm{H} 8+\mathrm{CH} 3<=>\mathrm{CH} 4+\mathrm{nC} 3 \mathrm{H} 7$

50. $\mathrm{C} 4 \mathrm{H} 81+\mathrm{H}(+\mathrm{M})<=>$ pC4H9 $(+\mathrm{M})$
$3.70 \mathrm{E}+24 \quad-2.9 \quad 12505.0$

$\begin{array}{lll}1.80 \mathrm{E}+12 & 0.0 & 0.0\end{array}$

$\begin{array}{lll}1.10 \mathrm{E}+13 & 0.0 & 0.0\end{array}$

$\begin{array}{lll}1.30 \mathrm{E}+06 & 2.5 & 6756.0\end{array}$

$\begin{array}{lll}9.03 \mathrm{E}-01 & 3.6 & 7153.0\end{array}$

$\begin{array}{lll}1.33 E+13 & 0.0 & 3260.7\end{array}$

$0.70000 \mathrm{E}+04$

TROE centering:

$0.10000 \mathrm{E}+01$

$0.10000 \mathrm{E}+04$

$0.13100 \mathrm{E}+04$

$0.48100 \mathrm{E}+05$
$\mathrm{H} 2$

CH4

$\mathrm{C} 2 \mathrm{H} 6$
Enhanced by $2.000 \mathrm{E}+00$

Enhanced by $2.000 \mathrm{E}+00$

Enhanced by

$3.000 E+00$
51. $\mathrm{C} 4 \mathrm{H} 81+\mathrm{H}<=>\mathrm{C} 2 \mathrm{H} 4+\mathrm{C} 2 \mathrm{H} 5$

52. $\mathrm{C} 4 \mathrm{H} 81+\mathrm{H}<=>\mathrm{C} 3 \mathrm{H} 6+\mathrm{CH} 3$

53. $\mathrm{C} 2 \mathrm{H} 4+\mathrm{C} 2 \mathrm{H} 5<=>\mathrm{pC} 4 \mathrm{H} 9$

54. $\mathrm{pC} 4 \mathrm{H} 9+\mathrm{H}<=>2 \mathrm{C} 2 \mathrm{H} 5$

55. $\mathrm{pC} 4 \mathrm{H} 9+\mathrm{H}<=>\mathrm{C} 4 \mathrm{H} 81+\mathrm{H} 2$

56. $\mathrm{pC} 4 \mathrm{H} 9+\mathrm{CH} 3<=>\mathrm{C} 4 \mathrm{H} 81+\mathrm{CH} 4$

57. $\mathrm{SXC} 5 \mathrm{H} 9<=>\mathrm{C} 3 \mathrm{H} 6+\mathrm{C} 2 \mathrm{H} 3$

58. $\mathrm{C} 5 \mathrm{H} 10<=>\mathrm{C} 2 \mathrm{H} 5+\mathrm{aC} 3 \mathrm{H} 5$ $\begin{array}{lll}1.39 \mathrm{E}+22 & -2.4 & 11180.0\end{array}$

$\begin{array}{lll}3.52 \mathrm{E}+22 & -2.4 & 11180.0\end{array}$

$\begin{array}{lll}1.50 \mathrm{E}+11 & 0.0 & 7300.0\end{array}$

$3.70 \mathrm{E}+24 \quad-2.9 \quad 12505.0$

$\begin{array}{lll}1.80 \mathrm{E}+12 & 0.0 & 0.0\end{array}$

$\begin{array}{lll}1.10 \mathrm{E}+13 & 0.0 & 0.0\end{array}$

$\begin{array}{lll}1.39 \mathrm{E}+12 & -0.6 & 37797.0\end{array}$

$\begin{array}{lll}7.31 \mathrm{E}+22 & -1.9 & 75470.0\end{array}$ 
59. $\mathrm{C} 5 \mathrm{H} 10<=>\mathrm{C} 3 \mathrm{H} 6+\mathrm{C} 2 \mathrm{H} 4$

60. $\mathrm{C} 5 \mathrm{H} 10+\mathrm{H}<=>\mathrm{C} 2 \mathrm{H} 4+\mathrm{nC} 3 \mathrm{H} 7$

61. $\mathrm{C} 5 \mathrm{H} 10+\mathrm{H}<=>\mathrm{C} 3 \mathrm{H} 6+\mathrm{C} 2 \mathrm{H} 5$

62. $\mathrm{C} 5 \mathrm{H} 10+\mathrm{H}<=>\mathrm{SXC} 5 \mathrm{H} 9+\mathrm{H} 2$

63. $\operatorname{PXC} 5 \mathrm{H} 11(+\mathrm{M})<=>\mathrm{C} 2 \mathrm{H} 4+\mathrm{nC} 3 \mathrm{H} 7(+\mathrm{M})$
$1.62 \mathrm{E}+06 \quad 1.8 \quad 53454.0$

$8.00 \mathrm{E}+21-2.4 \quad 11180.0$

$1.60 \mathrm{E}+22-2.4 \quad 11180.0$

$1.30 \mathrm{E}+06 \quad 2.4 \quad 4471.0$

$1.07 \mathrm{E}+13 \quad 0.0 \quad 28366.4$

Low pressure limit: $\quad 0.75690 \mathrm{E}-34 \quad 0.15410 \mathrm{E}+02-0.60000 \mathrm{E}+03$

TROE centering: $\quad-0.59100 \mathrm{E}+01 \quad 0.33300 \mathrm{E}+03 \quad 0.28000 \mathrm{E}+02 \quad 0.50000 \mathrm{E}+05$

$\begin{array}{lll}\mathrm{H} 2 & \text { Enhanced by } & 2.000 \mathrm{E}+00 \\ \mathrm{CH} 4 & \text { Enhanced by } & 2.000 \mathrm{E}+00 \\ \mathrm{C} 2 \mathrm{H} 6 & \text { Enhanced by } & 3.000 \mathrm{E}+00\end{array}$

64. $\operatorname{SXC} 5 \mathrm{H} 11(+\mathrm{M})<=>\mathrm{C} 3 \mathrm{H} 6+\mathrm{C} 2 \mathrm{H} 5(+\mathrm{M})$

$8.00 \mathrm{E}+12 \quad 0.0 \quad 27392.8$

Low pressure limit: $\quad 0.37000 \mathrm{E}-32 \quad 0.14910 \mathrm{E}+02-0.60000 \mathrm{E}+03$

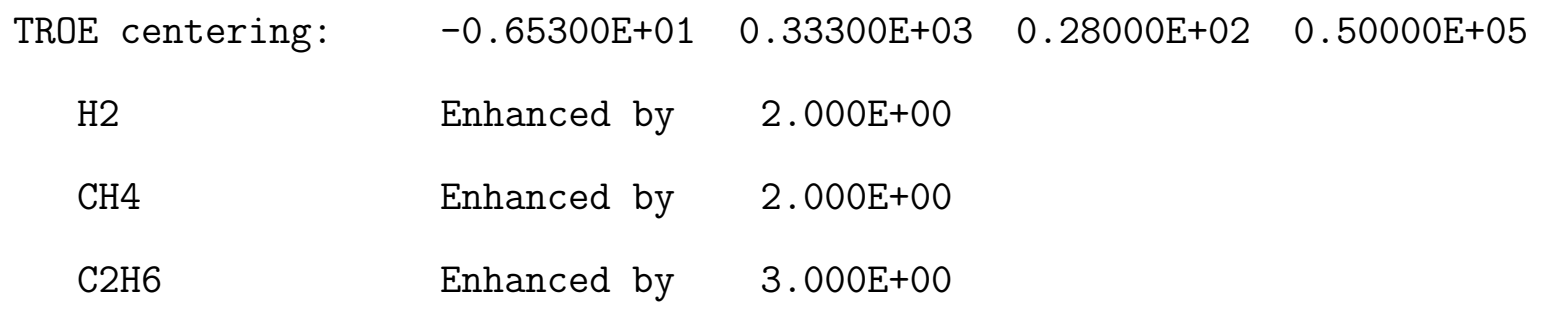

65. $\mathrm{SXC} 5 \mathrm{H} 11+\mathrm{H}<=>\mathrm{nC} 3 \mathrm{H} 7+\mathrm{C} 2 \mathrm{H} 5$

$\begin{array}{lll}1.40 \mathrm{E}+28 & -3.9 & 15916.0\end{array}$

66. $\mathrm{SXC} 5 \mathrm{H} 11+\mathrm{H}<=>\mathrm{C} 5 \mathrm{H} 10+\mathrm{H} 2$

$\begin{array}{lll}3.20 \mathrm{E}+12 & 0.0 & 0.0\end{array}$

67. $\mathrm{SXC} 5 \mathrm{H} 11+\mathrm{CH} 3<=>\mathrm{CH} 4+\mathrm{C} 5 \mathrm{H} 10$

$2.20 \mathrm{E}+14 \quad-0.7 \quad 0.0$

68. $\mathrm{PXC} 5 \mathrm{H} 11+\mathrm{H}<=>\mathrm{nC} 3 \mathrm{H} 7+\mathrm{C} 2 \mathrm{H} 5$

$3.70 \mathrm{E}+24 \quad-2.9 \quad 12505.0$

69. $\mathrm{PXC} 5 \mathrm{H} 11+\mathrm{H}<=>\mathrm{C} 5 \mathrm{H} 10+\mathrm{H} 2$

$\begin{array}{lll}1.80 \mathrm{E}+12 & 0.0 & 0.0\end{array}$

70. $\mathrm{PXC} 5 \mathrm{H} 11+\mathrm{CH} 3<=>\mathrm{C} 5 \mathrm{H} 10+\mathrm{CH} 4$

1. 10E+13

0.0

0.0

71. $\mathrm{C} 6 \mathrm{H} 12<=>\mathrm{aC} 3 \mathrm{H} 5+\mathrm{nC} 3 \mathrm{H} 7$

$1.03 E+23 \quad-2.0 \quad 74958.0$

72. $\mathrm{C} 6 \mathrm{H} 12<=>2 \mathrm{C} 3 \mathrm{H} 6$

$\begin{array}{lll}7.08 \mathrm{E}+06 & 1.6 & 53752.0\end{array}$

73. $\mathrm{C} 6 \mathrm{H} 12+\mathrm{H}<=>\mathrm{C} 2 \mathrm{H} 4+\mathrm{pC} 4 \mathrm{H} 9$

$8.00 \mathrm{E}+21-2.4 \quad 11180.0$

74. $\mathrm{C} 6 \mathrm{H} 12+\mathrm{H}<=>\mathrm{C} 3 \mathrm{H} 6+\mathrm{nC} 3 \mathrm{H} 7$

$1.60 \mathrm{E}+22-2.4 \quad 11180.0$

75. $\mathrm{PXC} 6 \mathrm{H} 13(+\mathrm{M})<=>\mathrm{C} 2 \mathrm{H} 4+\mathrm{pC} 4 \mathrm{H} 9(+\mathrm{M})$ $\begin{array}{lll}6.05 \mathrm{E}+11 & 0.3 & 27273.6\end{array}$ 
Low pressure limit: $0.42100 \mathrm{E}-34 \quad 0.15410 \mathrm{E}+02-0.60000 \mathrm{E}+03$

$\begin{array}{llll}\text { TROE centering: } \quad-0.59100 \mathrm{E}+01 & 0.33300 \mathrm{E}+03 & 0.28000 \mathrm{E}+02 & 0.50000 \mathrm{E}+05\end{array}$

$\begin{array}{lll}\text { H2 } & \text { Enhanced by } & 2.000 \mathrm{E}+00 \\ \mathrm{CH} 4 & \text { Enhanced by } & 2.000 \mathrm{E}+00 \\ \mathrm{C} 2 \mathrm{H} 6 & \text { Enhanced by } & 3.000 \mathrm{E}+00\end{array}$

76. $\operatorname{SXC6H13(+M)<=>C3H6+nC3H7(+M)} \quad 4.47 \mathrm{E}+11 \quad 0.6 \quad 28044.5$

Low pressure limit: $\quad 0.37000 \mathrm{E}-32 \quad 0.14910 \mathrm{E}+02-0.60000 \mathrm{E}+03$

$\begin{array}{llll}\text { TROE centering: } \quad-0.65300 \mathrm{E}+01 & 0.33300 \mathrm{E}+03 & 0.28000 \mathrm{E}+02 & 0.50000 \mathrm{E}+05\end{array}$

H2 Enhanced by $2.000 \mathrm{E}+00$

CH4 Enhanced by 2.000E+00

C2H6 Enhanced by $3.000 \mathrm{E}+00$

77. $\mathrm{S} 2 \mathrm{XC} 6 \mathrm{H} 13(+\mathrm{M})<=>\mathrm{C} 2 \mathrm{H} 5+\mathrm{C} 4 \mathrm{H} 81(+\mathrm{M}) \quad 3.55 \mathrm{E}+12 \quad 0.3 \quad 28296.9$

Low pressure limit: $0.45000 \mathrm{E}-25 \quad 0.13090 \mathrm{E}+02-0.60050 \mathrm{E}+03$

TROE centering: $\quad-0.74000 \mathrm{E}+00 \quad 0.30800 \mathrm{E}+03 \quad 0.28000 \mathrm{E}+02 \quad 0.50000 \mathrm{E}+05$

H2 Enhanced by $2.000 \mathrm{E}+00$

CH4 Enhanced by $2.000 \mathrm{E}+00$

C2H6 Enhanced by $3.000 \mathrm{E}+00$

78. $\mathrm{S} 2 \mathrm{XC} 6 \mathrm{H} 13(+\mathrm{M})<=>\mathrm{C} 5 \mathrm{H} 10+\mathrm{CH} 3(+\mathrm{M}) \quad 8.13 \mathrm{E}+10 \quad 0.8 \quad 29648.0$

Low pressure limit: $0.40000 \mathrm{E}-38 \quad 0.16780 \mathrm{E}+02-0.60040 \mathrm{E}+03$

TROE centering: $\quad-0.70300 \mathrm{E}+01 \quad 0.31400 \mathrm{E}+03 \quad 0.28000 \mathrm{E}+02 \quad 0.50000 \mathrm{E}+05$

H2 Enhanced by $2.000 \mathrm{E}+00$

CH4 Enhanced by $2.000 \mathrm{E}+00$

C2H6 Enhanced by $3.000 \mathrm{E}+00$

79. $\mathrm{PXC} 6 \mathrm{H} 13+\mathrm{H}<=>\mathrm{pC} 4 \mathrm{H} 9+\mathrm{C} 2 \mathrm{H} 5$

80. $\mathrm{PXC} 6 \mathrm{H} 13+\mathrm{H}<=>\mathrm{C} 6 \mathrm{H} 12+\mathrm{H} 2$

81. $\mathrm{PXC} 6 \mathrm{H} 13+\mathrm{CH} 3<=>\mathrm{C} 6 \mathrm{H} 12+\mathrm{CH} 4$

82. $\mathrm{SXC} 6 \mathrm{H} 13+\mathrm{H}<=>\mathrm{pC} 4 \mathrm{H} 9+\mathrm{C} 2 \mathrm{H} 5$
$3.70 \mathrm{E}+24 \quad-2.9 \quad 12505.0$

$\begin{array}{lll}1.80 \mathrm{E}+12 & 0.0 & 0.0\end{array}$

$\begin{array}{lll}1.10 \mathrm{E}+13 & 0.0 & 0.0\end{array}$

$1.40 \mathrm{E}+28 \quad-3.9 \quad 15916.0$ 
83. $\mathrm{SXC} 6 \mathrm{H} 13+\mathrm{H}<=>\mathrm{C} 6 \mathrm{H} 12+\mathrm{H} 2$

84. $\mathrm{SXC} 6 \mathrm{H} 13+\mathrm{CH} 3<=>\mathrm{CH} 4+\mathrm{C} 6 \mathrm{H} 12$

85. $\mathrm{C} 7 \mathrm{H} 14<=>\mathrm{pC} 4 \mathrm{H} 9+\mathrm{aC} 3 \mathrm{H} 5$

86. $\mathrm{C} 7 \mathrm{H} 14<=>\mathrm{C} 4 \mathrm{H} 81+\mathrm{C} 3 \mathrm{H} 6$

87. $\mathrm{C} 7 \mathrm{H} 14+\mathrm{H}<=>\mathrm{C} 2 \mathrm{H} 4+\mathrm{PXC} 5 \mathrm{H} 11$

88. $\mathrm{C} 7 \mathrm{H} 14+\mathrm{H}<=>\mathrm{C} 3 \mathrm{H} 6+\mathrm{pC} 4 \mathrm{H} 9$

89. $\mathrm{PXC7H} 15(+\mathrm{M})<=>\mathrm{C} 2 \mathrm{H} 4+\mathrm{PXC} 5 \mathrm{H} 11(+\mathrm{M})$
$3.20 \mathrm{E}+12 \quad 0.0$

$2.20 E+14 \quad-0.7$

$1.07 \mathrm{E}+23 \quad-2.0 \quad 74958.0$

$\begin{array}{lll}7.08 \mathrm{E}+06 & 1.6 \quad 53752.0\end{array}$

$8.00 \mathrm{E}+21-2.4 \quad 11180.0$

$1.60 \mathrm{E}+22 \quad-2.4 \quad 11180.0$

$\begin{array}{lll}7.94 \mathrm{E}+11 & 0.3 \quad 27210.0\end{array}$

Low pressure limit: $\quad 0.28000 \mathrm{E}-43 \quad 0.18730 \mathrm{E}+02-0.60250 \mathrm{E}+03$

TROE centering: $\quad-0.14660 \mathrm{E}+02$

$0.21900 \mathrm{E}+03$

$0.28000 \mathrm{E}+02$

$0.50000 \mathrm{E}+05$

$\begin{array}{lll}\mathrm{H} 2 & \text { Enhanced by } & 2.000 \mathrm{E}+00 \\ \mathrm{CH} 4 & \text { Enhanced by } & 2.000 \mathrm{E}+00 \\ \mathrm{C} 2 \mathrm{H} 6 & \text { Enhanced by } & 3.000 \mathrm{E}+00\end{array}$

90. $\operatorname{SXC} 7 \mathrm{H} 15(+\mathrm{M})<=>$ pC4H9+C3H6 $(+\mathrm{M})$

$5.01 \mathrm{E}+11 \quad 0.6 \quad 28092.2$

Low pressure limit: $0.89000 \mathrm{E}-38$

$0.16930 \mathrm{E}+02-0.60250 \mathrm{E}+03$

TROE centering: $-0.25270 \mathrm{E}+02$

$0.22300 E+03$

$0.28000 \mathrm{E}+02$

$0.50000 \mathrm{E}+05$ $\mathrm{H} 2$ Enhanced by

$2.000 E+00$

$\mathrm{CH} 4$

Enhanced by

$2.000 \mathrm{E}+00$

C2H6

Enhanced by

$3.000 \mathrm{E}+00$
91. $\mathrm{S} 2 \mathrm{XC7H} 15(+\mathrm{M})<=>\mathrm{nC} 3 \mathrm{H} 7+\mathrm{C} 4 \mathrm{H} 81(+\mathrm{M})$

$2.92 \mathrm{E}+12 \quad 0.3 \quad 28257.1$

Low pressure limit: $0.19820 \mathrm{E}-37 \quad 0.16810 \mathrm{E}+02-0.60240 \mathrm{E}+03$

TROE centering: $\quad-0.20960 \mathrm{E}+02$

$0.22100 \mathrm{E}+03$

$0.28000 \mathrm{E}+02$

$0.50000 \mathrm{E}+05$

Enhanced by

$2.000 \mathrm{E}+00$

$\mathrm{CH} 4$

Enhanced by

$2.000 \mathrm{E}+00$

$\mathrm{C} 2 \mathrm{H} 6$

$3.000 E+00$

92. $\mathrm{S} 2 \mathrm{XC} 7 \mathrm{H} 15(+\mathrm{M})<=>\mathrm{C} 6 \mathrm{H} 12+\mathrm{CH} 3(+\mathrm{M})$

$\begin{array}{lll}1.12 \mathrm{E}+11 & 0.8 \quad 29401.6\end{array}$ Low pressure limit: $0.10790 \mathrm{E}-41 \quad 0.18000 \mathrm{E}+02-0.60240 \mathrm{E}+03$ TROE centering: $\quad-0.20940 \mathrm{E}+02 \quad 0.21700 \mathrm{E}+03 \quad 0.28000 \mathrm{E}+02 \quad 0.50000 \mathrm{E}+05$ 


$\begin{array}{lll}\mathrm{H} 2 & \text { Enhanced by } & 2.000 \mathrm{E}+00 \\ \mathrm{CH} 4 & \text { Enhanced by } & 2.000 \mathrm{E}+00 \\ \mathrm{C} 2 \mathrm{H} 6 & \text { Enhanced by } & 3.000 \mathrm{E}+00\end{array}$

\begin{tabular}{|c|c|c|c|c|c|c|}
\hline \multicolumn{3}{|c|}{$\mathrm{PXC} 7 \mathrm{H} 15+\mathrm{H}<=>\mathrm{PXC} 5 \mathrm{H} 11+\mathrm{C} 2 \mathrm{H} 5$} & $3.70 E+24$ & -2 & & 12505.0 \\
\hline \multicolumn{3}{|l|}{$\mathrm{PXC} 7 \mathrm{H} 15+\mathrm{H}<=>\mathrm{C} 7 \mathrm{H} 14+\mathrm{H} 2$} & $1.80 \mathrm{E}+12$ & & 0 & 0.0 \\
\hline \multicolumn{3}{|c|}{$\mathrm{PXC} 7 \mathrm{H} 15+\mathrm{CH} 3<=>\mathrm{C} 7 \mathrm{H} 14+\mathrm{CH} 4$} & 1. $10 \mathrm{E}+13$ & & 0 & 0.0 \\
\hline \multicolumn{3}{|c|}{$\mathrm{SXC7H} 15+\mathrm{H}<=>\mathrm{PXC} 5 \mathrm{H} 11+\mathrm{C} 2 \mathrm{H} 5$} & $1.40 \mathrm{E}+28$ & -3 & & 15916.0 \\
\hline \multicolumn{3}{|l|}{$\mathrm{SXC7H} 15+\mathrm{H}<=>\mathrm{C} 7 \mathrm{H} 14+\mathrm{H} 2$} & $3.20 \mathrm{E}+12$ & & 0 & 0.0 \\
\hline \multicolumn{3}{|c|}{$\mathrm{SXC7H} 15+\mathrm{CH} 3<=>\mathrm{CH} 4+\mathrm{C} 7 \mathrm{H} 14$} & $2.20 \mathrm{E}+14$ & -0 & & 0 . \\
\hline \multicolumn{3}{|l|}{$\mathrm{C} 8 \mathrm{H} 16<=>\mathrm{PXC5H} 11+\mathrm{aC} 3 \mathrm{H} 5$} & $1.07 \mathrm{E}+23$ & -2 & & 74958.0 \\
\hline \multicolumn{3}{|l|}{$\mathrm{C} 8 \mathrm{H} 16<=>\mathrm{C} 5 \mathrm{H} 10+\mathrm{C} 3 \mathrm{H} 6$} & $7.08 \mathrm{E}+06$ & & 6 & 53752.0 \\
\hline \multicolumn{3}{|c|}{$\mathrm{C} 8 \mathrm{H} 16+\mathrm{H}<=>\mathrm{C} 2 \mathrm{H} 4+\mathrm{PXC} 6 \mathrm{H} 13$} & $8.00 E+21$ & -2 & & 11180.0 \\
\hline \multicolumn{3}{|c|}{$\mathrm{C} 8 \mathrm{H} 16+\mathrm{H}<=>\mathrm{C} 3 \mathrm{H} 6+\mathrm{PXC} 5 \mathrm{H} 11$} & $1.60 \mathrm{E}+22$ & -2 & & 11180.0 \\
\hline \multicolumn{3}{|c|}{$\mathrm{PXC} 8 \mathrm{H} 17(+\mathrm{M})<=>\mathrm{C} 2 \mathrm{H} 4+\mathrm{PXC} 6 \mathrm{H} 13(+\mathrm{M})$} & $9.12 \mathrm{E}+11$ & & 3 & 27237.8 \\
\hline Low pressure limit: & $0.18000 E-56$ & $0.23460 \mathrm{E}+02$ & \multicolumn{4}{|c|}{$-0.60240 \mathrm{E}+03$} \\
\hline TROE centering: & $-0.24600 \mathrm{E}+01$ & $0.20600 \mathrm{E}+03$ & \multicolumn{4}{|c|}{$0.28000 \mathrm{E}+02 \quad 0.50000 \mathrm{E}+05$} \\
\hline $\mathrm{H} 2$ & Enhanced by & $2.000 \mathrm{E}+00$ & & & & \\
\hline $\mathrm{CH} 4$ & Enhanced by & $2.000 \mathrm{E}+00$ & & & & \\
\hline $\mathrm{C} 2 \mathrm{H} 6$ & Enhanced by & $3.000 E+00$ & & & & \\
\hline
\end{tabular}

104. $\mathrm{S} 2 \mathrm{XC} 8 \mathrm{H} 17(+\mathrm{M})<=>\mathrm{pC} 4 \mathrm{H} 9+\mathrm{C} 4 \mathrm{H} 81(+\mathrm{M})$

$2.04 \mathrm{E}+13 \quad 0.0 \quad 28493.6$

Low pressure limit: $\quad 0.30000 \mathrm{E}-42 \quad 0.18430 \mathrm{E}+02-0.60280 \mathrm{E}+03$

TROE centering: $\quad-0.34470 \mathrm{E}+02 \quad 0.20800 \mathrm{E}+03 \quad 0.28000 \mathrm{E}+02 \quad 0.50000 \mathrm{E}+05$

$\begin{array}{lll}\mathrm{H} 2 & \text { Enhanced by } & 2.000 \mathrm{E}+00 \\ \mathrm{CH} 4 & \text { Enhanced by } & 2.000 \mathrm{E}+00 \\ \mathrm{C} 2 \mathrm{H} 6 & \text { Enhanced by } & 3.000 \mathrm{E}+00\end{array}$

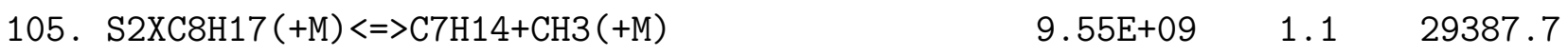

Low pressure limit: $\quad 0.53000 \mathrm{E}-45 \quad 0.19130 \mathrm{E}+02-0.60270 \mathrm{E}+03$ 
TROE centering: $\quad-0.34360 \mathrm{E}+02 \quad 0.21000 \mathrm{E}+03 \quad 0.28000 \mathrm{E}+02 \quad 0.50000 \mathrm{E}+05$

$\begin{array}{lll}\mathrm{H} 2 & \text { Enhanced by } & 2.000 \mathrm{E}+00 \\ \mathrm{CH} 4 & \text { Enhanced by } & 2.000 \mathrm{E}+00 \\ \mathrm{C} 2 \mathrm{H} 6 & \text { Enhanced by } & 3.000 \mathrm{E}+00\end{array}$

106. $\mathrm{S} 3 \mathrm{XC} 8 \mathrm{H} 17(+\mathrm{M})<=>\mathrm{nC} 3 \mathrm{H} 7+\mathrm{C} 5 \mathrm{H} 10(+\mathrm{M})$

$5.45 \mathrm{E}+11 \quad 0.6 \quad 28084.3$

Low pressure limit: $0.30720 \mathrm{E}-42 \quad 0.18420 \mathrm{E}+02-0.60290 \mathrm{E}+03$

TROE centering: $\quad-0.32130 \mathrm{E}+02 \quad 0.20700 \mathrm{E}+03 \quad 0.28000 \mathrm{E}+02 \quad 0.50000 \mathrm{E}+05$

H2 Enhanced by 2.000E+00

CH4 Enhanced by $2.000 \mathrm{E}+00$

C2H6 Enhanced by $\quad 3.000 \mathrm{E}+00$

107. $\mathrm{S} 3 \mathrm{XC} 8 \mathrm{H} 17(+\mathrm{M})<=>\mathrm{C} 6 \mathrm{H} 12+\mathrm{C} 2 \mathrm{H} 5(+\mathrm{M})$

$6.82 \mathrm{E}+09 \quad 1.1 \quad 27023.2$

Low pressure limit: $0.82740 \mathrm{E}-42 \quad 0.18280 \mathrm{E}+02-0.60290 \mathrm{E}+03$

TROE centering: $\quad-0.30040 \mathrm{E}+02 \quad 0.21000 \mathrm{E}+03 \quad 0.28000 \mathrm{E}+02 \quad 0.50000 \mathrm{E}+05$

H2 Enhanced by $2.000 \mathrm{E}+00$

CH4 Enhanced by 2.000E+00

C2H6 Enhanced by $\quad 3.000 \mathrm{E}+00$

108. $\mathrm{PXC} 8 \mathrm{H} 17+\mathrm{H}<=>\mathrm{PXC} 6 \mathrm{H} 13+\mathrm{C} 2 \mathrm{H} 5$

$3.70 \mathrm{E}+24 \quad-2.9 \quad 12505.0$

109. $\mathrm{PXC} 8 \mathrm{H} 17+\mathrm{H}<=>\mathrm{C} 8 \mathrm{H} 16+\mathrm{H} 2$

$\begin{array}{lll}1.80 \mathrm{E}+12 & 0.0 & 0.0\end{array}$

110. $\mathrm{PXC} 8 \mathrm{H} 17+\mathrm{CH} 3<=>\mathrm{C} 8 \mathrm{H} 16+\mathrm{CH} 4$

$1.10 \mathrm{E}+13 \quad 0.0 \quad 0.0$

111. $\mathrm{C} 9 \mathrm{H} 18<=>\mathrm{PXC} 6 \mathrm{H} 13+\mathrm{aC} 3 \mathrm{H} 5$

$1.07 \mathrm{E}+23-2.0 \quad 74958.0$

112. $\mathrm{C} 9 \mathrm{H} 18<=>\mathrm{C} 6 \mathrm{H} 12+\mathrm{C} 3 \mathrm{H} 6$

$7.08 \mathrm{E}+06 \quad 1.6 \quad 53752.0$

113. $\mathrm{C} 9 \mathrm{H} 18+\mathrm{H}<=>\mathrm{C} 2 \mathrm{H} 4+\mathrm{PXC} 7 \mathrm{H} 15$

$8.00 \mathrm{E}+21-2.4 \quad 11180.0$

114. $\mathrm{C} 9 \mathrm{H} 18+\mathrm{H}<=>\mathrm{C} 3 \mathrm{H} 6+\mathrm{PXC} 6 \mathrm{H} 13$

$\begin{array}{lll}1.60 \mathrm{E}+22 & -2.4 & 11180.0\end{array}$

115. $\mathrm{PXC} 9 \mathrm{H} 19(+\mathrm{M})<=>\mathrm{C} 2 \mathrm{H} 4+\mathrm{PXC} 7 \mathrm{H} 15(+\mathrm{M})$

$\begin{array}{lll}9.12 \mathrm{E}+11 & 0.3 & 27237.8\end{array}$

Low pressure limit: $0.18000 \mathrm{E}-56 \quad 0.23460 \mathrm{E}+02-0.60240 \mathrm{E}+03$

TROE centering: $\quad-0.24600 \mathrm{E}+01 \quad 0.20600 \mathrm{E}+03 \quad 0.28000 \mathrm{E}+02 \quad 0.50000 \mathrm{E}+05$

H2 Enhanced by $2.000 \mathrm{E}+00$ 


$\begin{array}{lll}\text { CH4 } & \text { Enhanced by } & 2.000 \mathrm{E}+00 \\ \mathrm{C} 2 \mathrm{H} 6 & \text { Enhanced by } & 3.000 \mathrm{E}+00\end{array}$

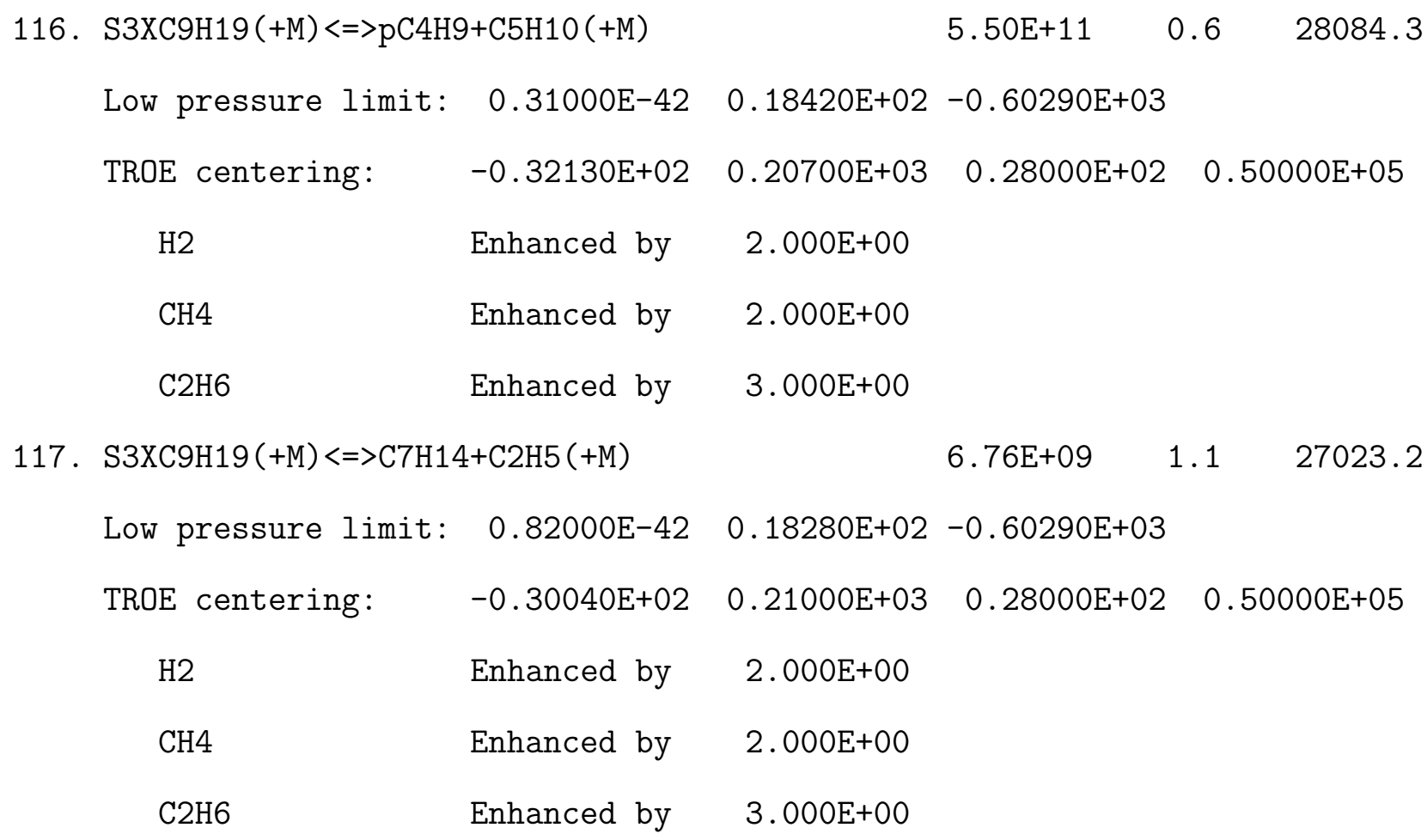

118. $\mathrm{S} 4 \mathrm{XC9H} 19(+\mathrm{M})<=>\mathrm{nC} 3 \mathrm{H} 7+\mathrm{C} 6 \mathrm{H} 12(+\mathrm{M})$

$1.10 \mathrm{E}+12 \quad 0.6 \quad 28084.3$

Low pressure limit: $\quad 0.62000 \mathrm{E}-42 \quad 0.18420 \mathrm{E}+02-0.60290 \mathrm{E}+03$

TROE centering: $\quad-0.32130 \mathrm{E}+02 \quad 0.20700 \mathrm{E}+03 \quad 0.28000 \mathrm{E}+02 \quad 0.50000 \mathrm{E}+05$

H2 Enhanced by $2.000 \mathrm{E}+00$

CH4 Enhanced by 2.000E+00

C2H6 Enhanced by 3.000E+00

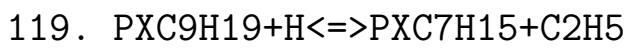

120. $\mathrm{PXC} 9 \mathrm{H} 19+\mathrm{H}<=>\mathrm{C} 9 \mathrm{H} 18+\mathrm{H} 2$

121. $\mathrm{PXC9H} 19+\mathrm{CH} 3<=>\mathrm{C} 9 \mathrm{H} 18+\mathrm{CH} 4$

122. $\mathrm{C} 10 \mathrm{H} 20<=>\mathrm{PXC} 7 \mathrm{H} 15+\mathrm{aC} 3 \mathrm{H} 5$

123. $\mathrm{C} 10 \mathrm{H} 2 \mathrm{O}<=>\mathrm{C} 7 \mathrm{H} 14+\mathrm{C} 3 \mathrm{H} 6$

124. $\mathrm{C} 1 \mathrm{OH} 2 \mathrm{O}+\mathrm{H}<=>\mathrm{C} 2 \mathrm{H} 4+\mathrm{PXC} 8 \mathrm{H} 17$

125. $\mathrm{C} 1 \mathrm{OH} 2 \mathrm{O}+\mathrm{H}<=>\mathrm{C} 3 \mathrm{H} 6+\mathrm{PXC} 7 \mathrm{H} 15$
$3.70 \mathrm{E}+24 \quad-2.9 \quad 12505.0$

$\begin{array}{lll}1.80 \mathrm{E}+12 & 0.0 & 0.0\end{array}$

$\begin{array}{lll}1.10 \mathrm{E}+13 & 0.0 & 0.0\end{array}$

$1.07 \mathrm{E}+23 \quad-2.0 \quad 74958.0$

$7.08 \mathrm{E}+06 \quad 1.6 \quad 53752.0$

8.00E+21 $-2.4 \quad 11180.0$

$\begin{array}{lll}1.60 \mathrm{E}+22 & -2.4 \quad 11180.0\end{array}$ 
126. $\mathrm{PXC} 10 \mathrm{H} 21(+\mathrm{M})<=>\mathrm{C} 2 \mathrm{H} 4+\mathrm{PXC} 8 \mathrm{H} 17(+\mathrm{M})$

$9.12 \mathrm{E}+11 \quad 0.3 \quad 27237.8$

Low pressure limit: $0.18000 \mathrm{E}-56 \quad 0.23460 \mathrm{E}+02-0.60240 \mathrm{E}+03$

$\begin{array}{lllll}\text { TROE centering: } & -0.24600 \mathrm{E}+01 & 0.20600 \mathrm{E}+03 & 0.28000 \mathrm{E}+02 & 0.50000 \mathrm{E}+05\end{array}$

H2 Enhanced by 2.000E+00

CH4 Enhanced by $2.000 \mathrm{E}+00$

C2H6 Enhanced by $3.000 \mathrm{E}+00$

127. $\mathrm{PXC} 10 \mathrm{H} 21+\mathrm{H}<=>\mathrm{PXC} 8 \mathrm{H} 17+\mathrm{C} 2 \mathrm{H} 5$

128. $\mathrm{PXC} 10 \mathrm{H} 21+\mathrm{H}<=>\mathrm{C} 10 \mathrm{H} 20+\mathrm{H} 2$

129. $\mathrm{PXC} 10 \mathrm{H} 21+\mathrm{CH} 3<=>\mathrm{C} 10 \mathrm{H} 2 \mathrm{O}+\mathrm{CH} 4$

130. $\mathrm{C} 11 \mathrm{H} 22<=>\mathrm{PXC} 8 \mathrm{H} 17+\mathrm{aC} 3 \mathrm{H} 5$

131. $\mathrm{C} 11 \mathrm{H} 22<=>\mathrm{C} 8 \mathrm{H} 16+\mathrm{C} 3 \mathrm{H} 6$

132. $\mathrm{C} 11 \mathrm{H} 22+\mathrm{H}<=>\mathrm{C} 2 \mathrm{H} 4+\mathrm{PXC} 9 \mathrm{H} 19$

133. $\mathrm{C} 11 \mathrm{H} 22+\mathrm{H}<=>\mathrm{C} 3 \mathrm{H} 6+\mathrm{PXC} 8 \mathrm{H} 17$

134. $\mathrm{PXC} 11 \mathrm{H} 23(+\mathrm{M})<=>\mathrm{C} 2 \mathrm{H} 4+\mathrm{PXC} 9 \mathrm{H} 19(+\mathrm{M})$ $\begin{array}{lll}3.70 \mathrm{E}+24 & -2.9 & 12505.0\end{array}$

$\begin{array}{lll}1.80 \mathrm{E}+12 & 0.0 & 0.0\end{array}$

$\begin{array}{lll}1.10 \mathrm{E}+13 & 0.0 & 0.0\end{array}$

$1.07 \mathrm{E}+23 \quad-2.0 \quad 74958.0$

$\begin{array}{lll}7.08 \mathrm{E}+06 & 1.6 \quad 53752.0\end{array}$

$8.00 \mathrm{E}+21 \quad-2.4 \quad 11180.0$

$\begin{array}{lll}1.60 \mathrm{E}+22 & -2.4 & 11180.0\end{array}$

$\begin{array}{lll}9.12 \mathrm{E}+11 & 0.3 & 27237.8\end{array}$

$0.23460 \mathrm{E}+02-0.60240 \mathrm{E}+03$

$0.20600 \mathrm{E}+03 \quad 0.28000 \mathrm{E}+02 \quad 0.50000 \mathrm{E}+05$
$0.18000 \mathrm{E}-56$ $-0.24600 \mathrm{E}+01$

$\mathrm{H} 2$

$\mathrm{CH} 4$

$\mathrm{C} 2 \mathrm{H} 6$
Enhanced by

Enhanced by

Enhanced by

$2.000 \mathrm{E}+00$

$2.000 \mathrm{E}+00$

$3.000 \mathrm{E}+00$

135. $\mathrm{PXC} 11 \mathrm{H} 23+\mathrm{H}<=>\mathrm{PXC} 9 \mathrm{H} 19+\mathrm{C} 2 \mathrm{H} 5$

136. $\mathrm{PXC} 11 \mathrm{H} 23+\mathrm{H}<=>\mathrm{C} 11 \mathrm{H} 22+\mathrm{H} 2$

137. $\mathrm{PXC} 11 \mathrm{H} 23+\mathrm{CH} 3<=>\mathrm{C} 11 \mathrm{H} 22+\mathrm{CH} 4$

138. $\mathrm{PXC} 12 \mathrm{H} 25(+\mathrm{M})<=>\mathrm{C} 2 \mathrm{H} 4+\mathrm{PXC} 10 \mathrm{H} 21(+\mathrm{M})$
$3.70 \mathrm{E}+24 \quad-2.9 \quad 12505.0$

$\begin{array}{lll}1.80 \mathrm{E}+12 & 0.0 & 0.0\end{array}$

$\begin{array}{lll}1.10 \mathrm{E}+13 & 0.0 & 0.0\end{array}$

$9.12 \mathrm{E}+11 \quad 0.3 \quad 27237.8$

Low pressure limit: $\quad 0.18000 \mathrm{E}-56 \quad 0.23460 \mathrm{E}+02-0.60240 \mathrm{E}+03$

TROE centering: $\quad-0.24600 \mathrm{E}+01$

$0.20600 \mathrm{E}+03$

$0.28000 \mathrm{E}+02$

$0.50000 \mathrm{E}+05$

$\mathrm{H} 2$

Enhanced by $2.000 \mathrm{E}+00$

$\mathrm{CH} 4$

Enhanced by

$2.000 \mathrm{E}+00$ 


\begin{tabular}{|c|c|c|c|c|c|c|}
\hline & $\mathrm{C} 2 \mathrm{H} 6$ & Enhanced by & $3.000 \mathrm{E}+00$ & & & \\
\hline 139. & $\mathrm{SXC} 12 \mathrm{H} 25(+\mathrm{M})<=>\mathrm{C} 3 \mathrm{H} 6$ & +PXC9H19 (+M) & & $6.03 E+10$ & 0.8 & 27820.0 \\
\hline & Low pressure limit: & $0.10000 \mathrm{E}-42$ & $0.18590 \mathrm{E}+02$ & $-0.60250 \mathrm{E}+03$ & & \\
\hline & TROE centering: & $-0.43320 \mathrm{E}+02$ & $0.20000 \mathrm{E}+03$ & $0.28000 \mathrm{E}+02$ & & $.0000 E+05$ \\
\hline & H2 & Enhanced by & $2.000 \mathrm{E}+00$ & & & \\
\hline & $\mathrm{CH} 4$ & Enhanced by & $2.000 \mathrm{E}+00$ & & & \\
\hline & $\mathrm{C} 2 \mathrm{H} 6$ & Enhanced by & $3.000 \mathrm{E}+00$ & & & \\
\hline 140. & $\mathrm{~S} 2 \mathrm{XC} 12 \mathrm{H} 25(+\mathrm{M})<=>\mathrm{C} 4 \mathrm{H}$ & $81+\mathrm{PXC} 8 \mathrm{H} 17(+\mathrm{M})$ & & $2.04 E+13$ & 0.0 & 28493.6 \\
\hline & Low pressure limit: & $0.30000 \mathrm{E}-42$ & $0.18430 \mathrm{E}+02$ & $-0.60280 E+03$ & & \\
\hline & TROE centering: & $-0.34470 \mathrm{E}+02$ & $0.20800 \mathrm{E}+03$ & $0.28000 \mathrm{E}+02$ & 20. & $0000 E+05$ \\
\hline & $\mathrm{H} 2$ & Enhanced by & $2.000 \mathrm{E}+00$ & & & \\
\hline & $\mathrm{CH} 4$ & Enhanced by & $2.000 \mathrm{E}+00$ & & & \\
\hline & $\mathrm{C} 2 \mathrm{H} 6$ & Enhanced by & $3.000 \mathrm{E}+00$ & & & \\
\hline 141. & $\mathrm{~S} 2 \mathrm{XC} 12 \mathrm{H} 25(+\mathrm{M})<=>\mathrm{C} 11$ & $\mathrm{H} 22+\mathrm{CH} 3(+\mathrm{M})$ & & $9.55 \mathrm{E}+09$ & 1.1 & 29387.7 \\
\hline & Low pressure limit: & $0.53000 \mathrm{E}-45$ & $0.19130 \mathrm{E}+02$ & $-0.60270 \mathrm{E}+03$ & & \\
\hline & TROE centering: & $-0.34360 \mathrm{E}+02$ & $0.21000 \mathrm{E}+03$ & $0.28000 \mathrm{E}+02$ & 20. & $0000 E+05$ \\
\hline & H2 & Enhanced by & $2.000 \mathrm{E}+00$ & & & \\
\hline & $\mathrm{CH} 4$ & Enhanced by & $2.000 \mathrm{E}+00$ & & & \\
\hline & $\mathrm{C} 2 \mathrm{H} 6$ & Enhanced by & $3.000 \mathrm{E}+00$ & & & \\
\hline 142. & $\mathrm{~S} 3 \mathrm{XC} 12 \mathrm{H} 25(+\mathrm{M})<=>\mathrm{C} 5 \mathrm{H}$ & 10+PXC7H15(+M) & & $5.50 \mathrm{E}+11$ & 0.6 & 28084.3 \\
\hline & Low pressure limit: & $0.31000 \mathrm{E}-42$ & $0.18420 \mathrm{E}+02$ & $-0.60290 \mathrm{E}+03$ & & \\
\hline & TROE centering: & $-0.32130 \mathrm{E}+02$ & $0.20700 \mathrm{E}+03$ & $0.28000 \mathrm{E}+02$ & & $0000 E+05$ \\
\hline & H2 & Enhanced by & $2.000 \mathrm{E}+00$ & & & \\
\hline & $\mathrm{CH} 4$ & Enhanced by & $2.000 \mathrm{E}+00$ & & & \\
\hline & $\mathrm{C} 2 \mathrm{H} 6$ & Enhanced by & $3.000 \mathrm{E}+00$ & & & \\
\hline 143. & $\mathrm{~S} 3 \mathrm{XC} 12 \mathrm{H} 25(+\mathrm{M})<=>\mathrm{C} 10$ & $\mathrm{H} 20+\mathrm{C} 2 \mathrm{H} 5(+\mathrm{M})$ & & $6.76 \mathrm{E}+09$ & 1.1 & 27023.2 \\
\hline
\end{tabular}


TROE centering: $\quad-0.30040 \mathrm{E}+02 \quad 0.21000 \mathrm{E}+03 \quad 0.28000 \mathrm{E}+02 \quad 0.50000 \mathrm{E}+05$

$\begin{array}{lll}\mathrm{H} 2 & \text { Enhanced by } & 2.000 \mathrm{E}+00 \\ \mathrm{CH} 4 & \text { Enhanced by } & 2.000 \mathrm{E}+00 \\ \mathrm{C} 2 \mathrm{H} 6 & \text { Enhanced by } & 3.000 \mathrm{E}+00\end{array}$

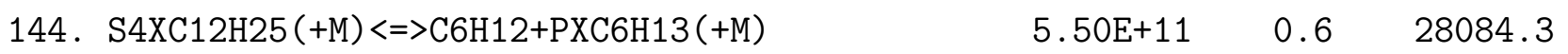

Low pressure limit: $0.31000 \mathrm{E}-42 \quad 0.18420 \mathrm{E}+02-0.60290 \mathrm{E}+03$

TROE centering: $\quad-0.32130 \mathrm{E}+02 \quad 0.20700 \mathrm{E}+03 \quad 0.28000 \mathrm{E}+02 \quad 0.50000 \mathrm{E}+05$

$\begin{array}{lll}\mathrm{H} 2 & \text { Enhanced by } & 2.000 \mathrm{E}+00 \\ \mathrm{CH} 4 & \text { Enhanced by } & 2.000 \mathrm{E}+00 \\ \mathrm{C} 2 \mathrm{H} 6 & \text { Enhanced by } & 3.000 \mathrm{E}+00\end{array}$

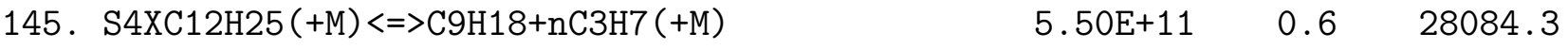

Low pressure limit: $\quad 0.31000 \mathrm{E}-42 \quad 0.18420 \mathrm{E}+02-0.60290 \mathrm{E}+03$

TROE centering: $\quad-0.32130 \mathrm{E}+02 \quad 0.20700 \mathrm{E}+03 \quad 0.28000 \mathrm{E}+02 \quad 0.50000 \mathrm{E}+05$

H2 Enhanced by $2.000 \mathrm{E}+00$

CH4 Enhanced by $2.000 \mathrm{E}+00$

C2H6 Enhanced by $\quad 3.000 \mathrm{E}+00$

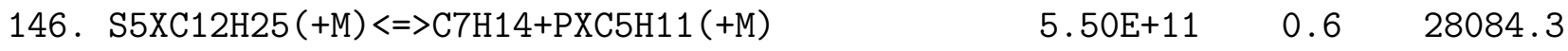

Low pressure limit: $0.31000 \mathrm{E}-42 \quad 0.18420 \mathrm{E}+02-0.60290 \mathrm{E}+03$

TROE centering: $\quad-0.32130 \mathrm{E}+02 \quad 0.20700 \mathrm{E}+03 \quad 0.28000 \mathrm{E}+02 \quad 0.50000 \mathrm{E}+05$

H2 Enhanced by $2.000 \mathrm{E}+00$

CH4 Enhanced by 2.000E+00

C2H6 Enhanced by $\quad 3.000 \mathrm{E}+00$

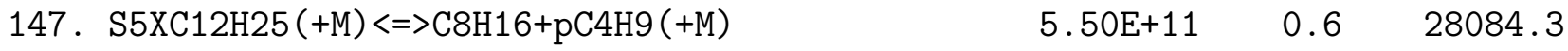

Low pressure limit: $\quad 0.31000 \mathrm{E}-42 \quad 0.18420 \mathrm{E}+02-0.60290 \mathrm{E}+03$

TROE centering: $\quad-0.32130 \mathrm{E}+02 \quad 0.20700 \mathrm{E}+03 \quad 0.28000 \mathrm{E}+02 \quad 0.50000 \mathrm{E}+05$

H2 Enhanced by $2.000 \mathrm{E}+00$

CH4 Enhanced by $2.000 \mathrm{E}+00$ 
C2H6 Enhanced by $3.000 \mathrm{E}+00$

148. $\mathrm{PXC12H} 25+\mathrm{H}(+\mathrm{M})<=>\mathrm{NC} 12 \mathrm{H} 26(+\mathrm{M})$

Low pressure limit: $\quad 0.30100 \mathrm{E}+49-0.93200 \mathrm{E}+01$

TROE centering:

$0.49800 \mathrm{E}+00$

$0.13140 E+04$

Enhanced by

$2.000 \mathrm{E}+00$

$\mathrm{CH} 4$

$\mathrm{C} 2 \mathrm{H} 6$

Enhanced by

Enhanced by
$2.000 E+00$

$3.000 \mathrm{E}+00$
149. $\mathrm{SXC} 12 \mathrm{H} 25+\mathrm{H}<=>\mathrm{PXC} 10 \mathrm{H} 21+\mathrm{C} 2 \mathrm{H} 5$

150. $\mathrm{PXC} 12 \mathrm{H} 25(+\mathrm{M})<=>\mathrm{S} 3 X \mathrm{C} 12 \mathrm{H} 25(+\mathrm{M})$

Low pressure limit: $0.51000 \mathrm{E}-43$

TROE centering: $\quad-0.20150 \mathrm{E}+02$

H2

$\mathrm{CH} 4$

$\mathrm{C} 2 \mathrm{H} 6$
Enhanced by

Enhanced by

Enhanced by $\begin{array}{lll}3.60 \mathrm{E}+13 & 0.0 & 0.0\end{array}$

$0.58340 \mathrm{E}+04$

$0.13140 \mathrm{E}+04 \quad 0.50000 \mathrm{E}+05$

151. $\mathrm{PXC9H} 19(+\mathrm{M})<=>\mathrm{S} 3 X C 9 H 19(+\mathrm{M})$

$1.40 \mathrm{E}+28 \quad-3.9 \quad 15916.0$

$\begin{array}{lll}5.13 \mathrm{E}+00 \quad 3.2 & 16847.8\end{array}$

$0.18750 \mathrm{E}+02-0.60290 \mathrm{E}+03$

$0.20500 \mathrm{E}+03 \quad 0.28000 \mathrm{E}+02 \quad 0.50000 \mathrm{E}+07$

Low pressure limit: $\quad 0.51000 \mathrm{E}-43 \quad 0.18750 \mathrm{E}+02-0.60290 \mathrm{E}+03$

TROE centering: $\quad-0.20150 \mathrm{E}+02 \quad 0.20500 \mathrm{E}+03 \quad 0.28000 \mathrm{E}+02 \quad 0.50000 \mathrm{E}+07$

H2 Enhanced by $2.000 \mathrm{E}+00$

CH4 Enhanced by 2.000E+00

C2H6 Enhanced by $3.000 \mathrm{E}+00$

Declared duplicate reaction...

152. $\operatorname{PXC} 8 \mathrm{H} 17(+\mathrm{M})<=>\mathrm{S} 3 X \mathrm{C} 8 \mathrm{H} 17(+\mathrm{M})$

$5.13 \mathrm{E}+00 \quad 3.2 \quad 16847.8$

Low pressure limit: $0.51000 \mathrm{E}-43 \quad 0.18750 \mathrm{E}+02-0.60290 \mathrm{E}+03$

TROE centering: $\quad-0.20150 \mathrm{E}+02 \quad 0.20500 \mathrm{E}+03 \quad 0.28000 \mathrm{E}+02 \quad 0.50000 \mathrm{E}+07$

$\begin{array}{lll}\mathrm{H} 2 & \text { Enhanced by } & 2.000 \mathrm{E}+00 \\ \mathrm{CH} 4 & \text { Enhanced by } & 2.000 \mathrm{E}+00 \\ \mathrm{C} 2 \mathrm{H} 6 & \text { Enhanced by } & 3.000 \mathrm{E}+00\end{array}$


Declared duplicate reaction...

153. $\mathrm{PXC6H13}(+\mathrm{M})<=>\mathrm{S} 2 X C 6 \mathrm{H} 13(+\mathrm{M})$

$6.73 \mathrm{E}+00 \quad 3.2 \quad 16557.7$

Low pressure limit: $0.19280 \mathrm{E}-25 \quad 0.12830 \mathrm{E}+02-0.60070 \mathrm{E}+03$

TROE centering: $\quad-0.10140 \mathrm{E}+02 \quad 0.30700 \mathrm{E}+03 \quad 0.28000 \mathrm{E}+02 \quad 0.50000 \mathrm{E}+07$

H2 Enhanced by $2.000 \mathrm{E}+00$

CH4 Enhanced by $2.000 \mathrm{E}+00$

C2H6 Enhanced by $\quad 3.000 \mathrm{E}+00$

154. $\operatorname{PXC5H} 11(+\mathrm{M})<=>\operatorname{SXC} 5 \mathrm{H} 11(+\mathrm{M})$

$9.29 \mathrm{E}+11 \quad 0.0 \quad 22453.1$

Low pressure limit: $0.18580 \mathrm{E}-25 \quad 0.12830 \mathrm{E}+02-0.60070 \mathrm{E}+03$

TROE centering: $\quad-0.10140 \mathrm{E}+02 \quad 0.30700 \mathrm{E}+03 \quad 0.28000 \mathrm{E}+02 \quad 0.50000 \mathrm{E}+07$

H2 Enhanced by $2.000 \mathrm{E}+00$

CH4 Enhanced by $2.000 \mathrm{E}+00$

C2H6 Enhanced by $\quad 3.000 \mathrm{E}+00$

155. $\mathrm{PXC} 12 \mathrm{H} 25(+\mathrm{M})<=>\mathrm{S} 4 \mathrm{XC} 12 \mathrm{H} 25(+\mathrm{M})$

$2.29 \mathrm{E}+01 \quad 2.8 \quad 10755.6$

Low pressure limit: $0.99000 \mathrm{E}-37 \quad 0.17210 \mathrm{E}+02-0.60300 \mathrm{E}+03$

TROE centering: $\quad-0.16330 \mathrm{E}+02 \quad 0.20000 \mathrm{E}+03 \quad 0.28000 \mathrm{E}+02 \quad 0.50000 \mathrm{E}+07$

H2 Enhanced by $2.000 \mathrm{E}+00$

CH4 Enhanced by $2.000 \mathrm{E}+00$

C2H6 Enhanced by $\quad 3.000 \mathrm{E}+00$

156. $\mathrm{PXC9H} 19(+\mathrm{M})<=>\mathrm{S} 4 \mathrm{XC9H} 19(+\mathrm{M})$

$2.29 \mathrm{E}+01 \quad 2.8 \quad 10755.6$

Low pressure limit: $0.99000 \mathrm{E}-37 \quad 0.17210 \mathrm{E}+02-0.60300 \mathrm{E}+03$

TROE centering: $\quad-0.16330 \mathrm{E}+02 \quad 0.20000 \mathrm{E}+03 \quad 0.28000 \mathrm{E}+02 \quad 0.50000 \mathrm{E}+07$

H2 Enhanced by $2.000 \mathrm{E}+00$

CH4 Enhanced by $2.000 \mathrm{E}+00$

C2H6 Enhanced by $\quad 3.000 \mathrm{E}+00$

157. $\mathrm{PXC} 8 \mathrm{H} 17(+\mathrm{M})<=>\mathrm{S} 3 X \mathrm{C} 8 \mathrm{H} 17(+\mathrm{M})$

$2.29 \mathrm{E}+01 \quad 2.8 \quad 10755.6$

Low pressure limit: $\quad 0.99000 \mathrm{E}-37 \quad 0.17210 \mathrm{E}+02-0.60300 \mathrm{E}+03$ 
TROE centering: $\quad-0.16330 \mathrm{E}+02 \quad 0.20000 \mathrm{E}+03 \quad 0.28000 \mathrm{E}+02 \quad 0.50000 \mathrm{E}+07$

$\begin{array}{lll}\text { H2 } & \text { Enhanced by } & 2.000 \mathrm{E}+00 \\ \mathrm{CH} 4 & \text { Enhanced by } & 2.000 \mathrm{E}+00 \\ \mathrm{C} 2 \mathrm{H} 6 & \text { Enhanced by } & 3.000 \mathrm{E}+00\end{array}$

Declared duplicate reaction...

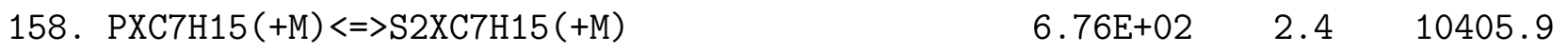

Low pressure limit: $\quad 0.54000 \mathrm{E}-25 \quad 0.13200 \mathrm{E}+02-0.60260 \mathrm{E}+03$

TROE centering: $\quad-0.25390 \mathrm{E}+02 \quad 0.21500 \mathrm{E}+03 \quad 0.28000 \mathrm{E}+02 \quad 0.50000 \mathrm{E}+07$

H2 Enhanced by $2.000 \mathrm{E}+00$

CH4 Enhanced by $2.000 \mathrm{E}+00$

C2H6 Enhanced by $3.000 \mathrm{E}+00$

159. $\mathrm{PXC6H13}(+\mathrm{M})<=>\mathrm{SXC} 6 \mathrm{H} 13(+\mathrm{M})$

$\begin{array}{lll}2.57 \mathrm{E}+02 & 2.5 & 10960.3\end{array}$

Low pressure limit: $0.75820 \mathrm{E}-25 \quad 0.13090 \mathrm{E}+02-0.60260 \mathrm{E}+03$

TROE centering: $\quad-0.25380 \mathrm{E}+02 \quad 0.21500 \mathrm{E}+03 \quad 0.28000 \mathrm{E}+02 \quad 0.50000 \mathrm{E}+07$

H2 Enhanced by $2.000 \mathrm{E}+00$

CH4 Enhanced by $2.000 \mathrm{E}+00$

C2H6 Enhanced by $\quad 3.000 \mathrm{E}+00$

160. $\mathrm{PXC12H} 25(+\mathrm{M})<=>\mathrm{S} 5 \mathrm{XC} 12 \mathrm{H} 25(+\mathrm{M})$

$2.95 \mathrm{E}+00 \quad 3.1 \quad 11015.9$

Low pressure limit: $0.39000 \mathrm{E}-33 \quad 0.15860 \mathrm{E}+02-0.60620 \mathrm{E}+03$

TROE centering: $\quad-0.15240 \mathrm{E}+02 \quad 0.21600 \mathrm{E}+03 \quad 0.28000 \mathrm{E}+02 \quad 0.50000 \mathrm{E}+07$

H2 Enhanced by $2.000 \mathrm{E}+00$

CH4 Enhanced by $2.000 \mathrm{E}+00$

C2H6 Enhanced by $\quad 3.000 \mathrm{E}+00$

161. $\mathrm{PXC9H} 19(+\mathrm{M})<=>\mathrm{S} 3 X C 9 H 19(+\mathrm{M})$

$2.95 \mathrm{E}+00 \quad 3.1 \quad 11015.9$

Low pressure limit: $0.39000 \mathrm{E}-33 \quad 0.15860 \mathrm{E}+02-0.60620 \mathrm{E}+03$

TROE centering: $\quad-0.15240 \mathrm{E}+02 \quad 0.21600 \mathrm{E}+03 \quad 0.28000 \mathrm{E}+02 \quad 0.50000 \mathrm{E}+07$

H2 Enhanced by $2.000 \mathrm{E}+00$ 


$\begin{array}{lll}\text { CH4 } & \text { Enhanced by } & 2.000 \mathrm{E}+00 \\ \mathrm{C} 2 \mathrm{H} 6 & \text { Enhanced by } & 3.000 \mathrm{E}+00\end{array}$

Declared duplicate reaction...

162. $\mathrm{PXC} 8 \mathrm{H} 17(+\mathrm{M})<=>\mathrm{S} 2 \mathrm{XC} 8 \mathrm{H} 17(+\mathrm{M})$

$2.95 \mathrm{E}+00 \quad 3.1 \quad 11015.9$

Low pressure limit: $0.39000 \mathrm{E}-33 \quad 0.15860 \mathrm{E}+02-0.60620 \mathrm{E}+03$

TROE centering: $\quad-0.15240 \mathrm{E}+02 \quad 0.21600 \mathrm{E}+03 \quad 0.28000 \mathrm{E}+02 \quad 0.50000 \mathrm{E}+07$

$\begin{array}{lll}\text { H2 } & \text { Enhanced by } & 2.000 \mathrm{E}+00 \\ \mathrm{CH} 4 & \text { Enhanced by } & 2.000 \mathrm{E}+00 \\ \mathrm{C} 2 \mathrm{H} 6 & \text { Enhanced by } & 3.000 \mathrm{E}+00\end{array}$

163. $\mathrm{PXC7H15}(+\mathrm{M})<=>\operatorname{SXC} 7 \mathrm{H} 15(+\mathrm{M})$

$2.45 \mathrm{E}+02 \quad 2.5 \quad 12502.2$

Low pressure limit: $0.33000 \mathrm{E}-29 \quad 0.14310 \mathrm{E}+02-0.60250 \mathrm{E}+03$

TROE centering: $\quad-0.27190 \mathrm{E}+02 \quad 0.22000 \mathrm{E}+03 \quad 0.28000 \mathrm{E}+02 \quad 0.50000 \mathrm{E}+07$

$\begin{array}{lll}\mathrm{H} 2 & \text { Enhanced by } & 2.000 \mathrm{E}+00 \\ \mathrm{CH} 4 & \text { Enhanced by } & 2.000 \mathrm{E}+00 \\ \mathrm{C} 2 \mathrm{H} 6 & \text { Enhanced by } & 3.000 \mathrm{E}+00\end{array}$

164. $\mathrm{S} 3 \mathrm{XC} 12 \mathrm{H} 25(+\mathrm{M})<=>\mathrm{S} 5 \mathrm{XC} 12 \mathrm{H} 25(+\mathrm{M})$

$1.41 \mathrm{E}+00 \quad 3.3 \quad 16144.4$

Low pressure limit: $0.52000 \mathrm{E}-29 \quad 0.14080 \mathrm{E}+02-0.60640 \mathrm{E}+03$

TROE centering: $\quad-0.21930 \mathrm{E}+02 \quad 0.21900 \mathrm{E}+03 \quad 0.28000 \mathrm{E}+02 \quad 0.50000 \mathrm{E}+07$

$\begin{array}{lll}\mathrm{H} 2 & \text { Enhanced by } & 2.000 \mathrm{E}+00 \\ \mathrm{CH} 4 & \text { Enhanced by } & 2.000 \mathrm{E}+00 \\ \mathrm{C} 2 \mathrm{H} 6 & \text { Enhanced by } & 3.000 \mathrm{E}+00\end{array}$

165. $\mathrm{S} 2 \mathrm{XC} 12 \mathrm{H} 25(+\mathrm{M})<=>\mathrm{S} 5 \mathrm{XC} 12 \mathrm{H} 25(+\mathrm{M})$

$1.41 \mathrm{E}+00 \quad 3.3 \quad 16144.4$

Low pressure limit: $0.52000 \mathrm{E}-29 \quad 0.14080 \mathrm{E}+02-0.60640 \mathrm{E}+03$

TROE centering: $\quad-0.21930 \mathrm{E}+02 \quad 0.21900 \mathrm{E}+03 \quad 0.28000 \mathrm{E}+02 \quad 0.50000 \mathrm{E}+07$

$\begin{array}{lll}\mathrm{H} 2 & \text { Enhanced by } & 2.000 \mathrm{E}+00 \\ \mathrm{CH} 4 & \text { Enhanced by } & 2.000 \mathrm{E}+00 \\ \mathrm{C} 2 \mathrm{H} 6 & \text { Enhanced by } & 3.000 \mathrm{E}+00\end{array}$


Declared duplicate reaction...

166. $\operatorname{SXC} 12 \mathrm{H} 25(+\mathrm{M})<=>\mathrm{S} 4 \mathrm{XC} 12 \mathrm{H} 25(+\mathrm{M})$

$1.41 \mathrm{E}+00 \quad 3.3 \quad 16144.4$

Low pressure limit: $\quad 0.52000 \mathrm{E}-29 \quad 0.14080 \mathrm{E}+02-0.60640 \mathrm{E}+03$

TROE centering: $\quad-0.21930 \mathrm{E}+02 \quad 0.21900 \mathrm{E}+03 \quad 0.28000 \mathrm{E}+02 \quad 0.50000 \mathrm{E}+07$

$\begin{array}{lll}\text { H2 } & \text { Enhanced by } & 2.000 \mathrm{E}+00 \\ \mathrm{CH} 4 & \text { Enhanced by } & 2.000 \mathrm{E}+00 \\ \mathrm{C} 2 \mathrm{H} 6 & \text { Enhanced by } & 3.000 \mathrm{E}+00\end{array}$

167. $\operatorname{SXC} 7 \mathrm{H} 15(+\mathrm{M})<=>\mathrm{S} 2 X C 7 \mathrm{H} 15(+\mathrm{M})$

$2.45 \mathrm{E}+01 \quad 3.1 \quad 18107.5$

Low pressure limit: $0.13000 \mathrm{E}-31 \quad 0.14830 \mathrm{E}+02-0.60260 \mathrm{E}+03$

TROE centering: $\quad-0.28410 \mathrm{E}+02 \quad 0.21900 \mathrm{E}+03 \quad 0.28000 \mathrm{E}+02 \quad 0.50000 \mathrm{E}+07$

H2 Enhanced by 2.000E+00

CH4 Enhanced by $2.000 \mathrm{E}+00$

C2H6 Enhanced by $\quad 3.000 \mathrm{E}+00$

168. $\operatorname{SXC12H25}(+\mathrm{M})<=>\operatorname{S} 5 X C 12 \mathrm{H} 25(+\mathrm{M})$

$1.86 \mathrm{E}+00 \quad 3.3 \quad 13197.7$

Low pressure limit: $0.30000 \mathrm{E}-26 \quad 0.13480 \mathrm{E}+02-0.60650 \mathrm{E}+03$

TROE centering: $\quad-0.19700 \mathrm{E}+02 \quad 0.21500 \mathrm{E}+03 \quad 0.28000 \mathrm{E}+02 \quad 0.50000 \mathrm{E}+07$

H2 Enhanced by $2.000 \mathrm{E}+00$

CH4 Enhanced by $2.000 \mathrm{E}+00$

C2H6 Enhanced by $3.000 \mathrm{E}+00$

Declared duplicate reaction...

169. $\mathrm{S} 2 \mathrm{XC} 12 \mathrm{H} 25(+\mathrm{M})<=>\mathrm{S} 5 \mathrm{XC} 12 \mathrm{H} 25(+\mathrm{M})$

$1.86 \mathrm{E}+00 \quad 3.3 \quad 13197.7$

Low pressure limit: $0.30000 \mathrm{E}-26 \quad 0.13480 \mathrm{E}+02-0.60650 \mathrm{E}+03$

TROE centering: $\quad-0.19700 \mathrm{E}+02 \quad 0.21500 \mathrm{E}+03 \quad 0.28000 \mathrm{E}+02 \quad 0.50000 \mathrm{E}+07$

H2 Enhanced by $2.000 \mathrm{E}+00$

CH4 Enhanced by 2.000E+00

C2H6 Enhanced by $3.000 \mathrm{E}+00$

Declared duplicate reaction... 
170. $\mathrm{S} 3 \mathrm{XC} 12 \mathrm{H} 25(+\mathrm{M})<=>\mathrm{S} 4 \mathrm{XC} 12 \mathrm{H} 25(+\mathrm{M})$

$1.86 \mathrm{E}+00 \quad 3.3 \quad 13197.7$

Low pressure limit: $0.30000 \mathrm{E}-26 \quad 0.13480 \mathrm{E}+02-0.60650 \mathrm{E}+03$

TROE centering: $\quad-0.19700 \mathrm{E}+02 \quad 0.21500 \mathrm{E}+03 \quad 0.28000 \mathrm{E}+02 \quad 0.50000 \mathrm{E}+07$

H2 Enhanced by 2.000E+00

CH4 Enhanced by $2.000 \mathrm{E}+00$

C2H6 Enhanced by $3.000 \mathrm{E}+00$

171. $\mathrm{S} 2 \mathrm{XC} 12 \mathrm{H} 25(+\mathrm{M})<=>\mathrm{S} 4 \mathrm{XC} 12 \mathrm{H} 25(+\mathrm{M})$

$2.95 \mathrm{E}+00 \quad 3.1 \quad 12865.9$

Low pressure limit: $0.39000 \mathrm{E}-33 \quad 0.15860 \mathrm{E}+02 \quad 0.12440 \mathrm{E}+04$

$\begin{array}{lllll}\text { TROE centering: } & -0.15240 \mathrm{E}+02 & 0.21600 \mathrm{E}+03 & 0.28000 \mathrm{E}+02 & 0.50000 \mathrm{E}+07\end{array}$

H2 Enhanced by 2.000E+00

CH4 Enhanced by $2.000 \mathrm{E}+00$

C2H6 Enhanced by $3.000 \mathrm{E}+00$

172. $\mathrm{SXC} 12 \mathrm{H} 25(+\mathrm{M})<=>\mathrm{S} 5 \mathrm{XC} 12 \mathrm{H} 25(+\mathrm{M})$

$2.95 \mathrm{E}+00 \quad 3.1 \quad 12865.9$

Low pressure limit: $0.39000 \mathrm{E}-33 \quad 0.15860 \mathrm{E}+02 \quad 0.12440 \mathrm{E}+04$

$\begin{array}{lllll}\text { TROE centering: } & -0.15240 \mathrm{E}+02 & 0.21600 \mathrm{E}+03 & 0.28000 \mathrm{E}+02 & 0.50000 \mathrm{E}+07\end{array}$

H2 Enhanced by 2.000E+00

CH4 Enhanced by $2.000 \mathrm{E}+00$

C2H6 Enhanced by $3.000 \mathrm{E}+00$

Declared duplicate reaction...

173. $\mathrm{PXC} 11 \mathrm{H} 23+\mathrm{CH} 3<=>\mathrm{NC} 12 \mathrm{H} 26$

174. $\mathrm{PXC} 10 \mathrm{H} 21+\mathrm{C} 2 \mathrm{H} 5<=>\mathrm{NC} 12 \mathrm{H} 26$

175. $\mathrm{PXC} 9 \mathrm{H} 19+\mathrm{nC} 3 \mathrm{H} 7<=>\mathrm{NC} 12 \mathrm{H} 26$

176. $\mathrm{PXC} 8 \mathrm{H} 17+\mathrm{pC} 4 \mathrm{H} 9<=>\mathrm{NC} 12 \mathrm{H} 26$

177. $\mathrm{PXC7H15+PXC5H11<=>NC12H26}$

178. $2 \mathrm{PXC6} 1 \mathrm{H} 13<=>\mathrm{NC} 12 \mathrm{H} 26$

179. $\mathrm{NC} 12 \mathrm{H} 26+\mathrm{H}<=>\mathrm{PXC} 12 \mathrm{H} 25+\mathrm{H} 2$

180. $\mathrm{NC} 12 \mathrm{H} 26+\mathrm{H}<=>\mathrm{SXC} 12 \mathrm{H} 25+\mathrm{H} 2$
$1.93 \mathrm{E}+14 \quad-0.3 \quad 0.0$

$\begin{array}{lll}1.65 E+14 & -0.5 & 0.0\end{array}$

$\begin{array}{lll}1.88 \mathrm{E}+14 & -0.5 & 0.0\end{array}$

$\begin{array}{lll}1.40 \mathrm{E}+14 & -0.5 & 0.0\end{array}$

$\begin{array}{lll}1.73 \mathrm{E}+14 & -0.5 & 0.0\end{array}$

$\begin{array}{lll}8.90 \mathrm{E}+14 & -0.5 & 0.0\end{array}$

$1.30 \mathrm{E}+06 \quad 2.5 \quad 6756.0$

$3.07 \mathrm{E}+06 \quad 2.4 \quad 4471.0$ 


\begin{abstract}
181. $\mathrm{NC} 12 \mathrm{H} 26+\mathrm{H}<=>\mathrm{S} 2 \mathrm{XC} 12 \mathrm{H} 25+\mathrm{H} 2$
182. $\mathrm{NC} 12 \mathrm{H} 26+\mathrm{H}<=>\mathrm{S} 3 \mathrm{XC} 12 \mathrm{H} 25+\mathrm{H} 2$

183. $\mathrm{NC} 12 \mathrm{H} 26+\mathrm{H}<=>\mathrm{S} 4 \mathrm{XC} 12 \mathrm{H} 25+\mathrm{H} 2$

184. $\mathrm{NC} 12 \mathrm{H} 26+\mathrm{H}<=>\mathrm{S} 5 \mathrm{XC} 12 \mathrm{H} 25+\mathrm{H} 2$

185. $\mathrm{NC} 12 \mathrm{H} 26+\mathrm{CH} 3<=>\mathrm{PXC} 12 \mathrm{H} 25+\mathrm{CH} 4$

186. $\mathrm{NC} 12 \mathrm{H} 26+\mathrm{CH} 3<=>\mathrm{SXC} 12 \mathrm{H} 25+\mathrm{CH} 4$

187. $\mathrm{NC} 12 \mathrm{H} 26+\mathrm{CH} 3<=>\mathrm{S} 2 \mathrm{XC} 12 \mathrm{H} 25+\mathrm{CH} 4$

188. $\mathrm{NC} 12 \mathrm{H} 26+\mathrm{CH} 3<=>\mathrm{S} 3 \mathrm{XC} 12 \mathrm{H} 25+\mathrm{CH} 4$

189. $\mathrm{NC} 12 \mathrm{H} 26+\mathrm{CH} 3<=>\mathrm{S} 4 \mathrm{XC} 12 \mathrm{H} 25+\mathrm{CH} 4$

190. $\mathrm{NC} 12 \mathrm{H} 26+\mathrm{CH} 3<=>\mathrm{S} 5 \mathrm{XC} 12 \mathrm{H} 25+\mathrm{CH} 4$
\end{abstract}

$\begin{array}{lll}3.49 \mathrm{E}+06 & 2.4 & 4471.0\end{array}$

$2.96 \mathrm{E}+06 \quad 2.4 \quad 4471.0$

$3.86 \mathrm{E}+06 \quad 2.4 \quad 4471.0$

$2.70 \mathrm{E}+06 \quad 2.4 \quad 4471.0$

$\begin{array}{lll}9.03 \mathrm{E}-01 & 3.6 & 7153.0\end{array}$

$\begin{array}{lll}3.00 \mathrm{E}+00 \quad 3.5 & 5480.0\end{array}$

$\begin{array}{lll}3.00 \mathrm{E}+00 \quad 3.5 & 5480.0\end{array}$

$\begin{array}{lll}3.00 \mathrm{E}+00 \quad 3.5 & 5480.0\end{array}$

$\begin{array}{lll}3.00 \mathrm{E}+00 \quad 3.5 & 5480.0\end{array}$

$3.00 \mathrm{E}+00 \quad 3.5 \quad 5480.0$

NOTE: A units mole-cm-sec-K, E units cal/mole 


\section{Bibliography}

[1] William H Heiser and David T Pratt. Hypersonic airbreathing propulsion. AIAA, 1994.

[2] Corin Segal. The Scramjet Engine: Processes and Characteristics. Cambridge Aerospace Series. Cambridge University Press, 2009.

[3] Antonio Ferri. Mixing-controlled supersonic combustion. Annual Review of Fluid Mechanics, 5(1):301-338, 1973.

[4] James C McDaniel, Harsha Chelliah, Christopher P Goyne, Jack R Edwards, Peyman Givi, and Andrew D Cutler. Us national center for hypersonic combined cycle propulsion: an overview. AIAA Paper, 7280, 2009.

[5] H Lander and AC Nixon. Endothermic fuels for hypersonic vehicles. Journal of Aircraft, 8(4):200-207, 1971.

[6] Tim Edwards and SD Anderson. Results of high temperature jp-7 cracking assessment. AIAA Paper, 806:1993, 1993.

[7] He Huang, David R Sobel, and Louis J Spadaccini. Endothermic heat-sink of hydrocarbon fuels for scramjet cooling. AIAA paper, 2002-3871.

[8] Tim Edwards. Cracking and deposition behavior of supercritical hydrocarbon aviation fuels. Combustion Science and Technology, 178(1-3):307-334, 2006.

[9] Hai Wang and Michael Frenklach. A detailed kinetic modeling study of aromatics formation in laminar premixed acetylene and ethylene flames. Combustion and flame, 110(1):173-221, 1997.

[10] Hai Wang. Formation of nascent soot and other condensed-phase materials in flames. Proceedings of the Combustion Institute, 33(1):41-67, 2011.

[11] Timothy J Held and Frederick L Dryer. A comprehensive mechanism for methanol oxidation. International Journal of Chemical Kinetics, 30(11):805830, 1998.

[12] MA Mueller, TJ Kim, RA Yetter, and FL Dryer. Flow reactor studies and kinetic modeling of the h2/o2 reaction. International Journal of Chemical Kinetics, 31(2):113-125, 1999. 
[13] Alexander Laskin, Hai Wang, and Chung K Law. Detailed kinetic modeling of 1 , 3-butadiene oxidation at high temperatures. International Journal of Chemical Kinetics, 32(10):589-614, 2000.

[14] PS Veloo, S Jahangirian, and FL Dryer. An experimental and kinetic modeling study of the two stage autoignition kinetic behavior of c7, c10, c12, and c14 nalkanes. In Spring Technical Meeting, Central States Section of the Combustion Institute, 2012.

[15] Saeed Jahangirian, Stephen Dooley, Francis M Haas, and Frederick L Dryer. A detailed experimental and kinetic modeling study of n-decane oxidation at elevated pressures. Combustion and Flame, 159(1):30-43, 2012.

[16] Matthew S Kurman, Robert H Natelson, Nicholas P Cernansky, and David L Miller. Speciation of the reaction intermediates from n-dodecane oxidation in the low temperature regime. Proceedings of the Combustion Institute, 33(1):159$166,2011$.

[17] Matthew S Kurman. The preignition oxidation chemistry of $n$-decane and $n$ dodecane in a pressurized flow reactor and their use as jet fuel surrogate components. PhD thesis, Drexel University, 2010.

[18] KM Walters and CT Bowman. Vitiated ethane oxidation in a high-pressure flow reactor. Combustion and Flame, 156(10):1886-1897, 2009.

[19] Sayak Banerjee, Rei Tangko, David A Sheen, Hai Wang, and C Tom Bowman. An experimental and kinetic modeling study of n-dodecane pyrolysis and oxidation. Combustion and Flame, 163:12-30, 2016.

[20] U. Shrestha, M. J. Rahimi, G. P. Simms, and H. K. Chelliah. Fuel pyrolysis in a microflow tube reactor measurement and modeling uncertainties of ethane, n-butane, and n-dodecane pyrolysis. Combustion and Flame (submitted), 2016.

[21] Andrew E Lutz, Robert J Kee, and James A Miller. Senkin: A fortran program for predicting homogeneous gas phase chemical kinetics with sensitivity analysis. Technical Report SAND-87-8248, Sandia National Labs., Livermore, CA, 1988 .

[22] Robert J Kee, Fran M Rupley, and James A Miller. Chemkin-ii: A fortran chemical kinetics package for the analysis of gas-phase chemical kinetics. Technical Report SAND89-8009B, Sandia National Labs., Livermore, CA, 1989.

[23] Robert J Kee, Joseph F Grcar, MD Smooke, JA Miller, and E Meeks. Premix: a fortran program for modeling steady laminar one-dimensional premixed flames. Technical Report SAND85-8240, Sandia National Labs., Livermore, CA, 1985.

[24] Geoffrey Taylor. Dispersion of soluble matter in solvent flowing slowly through a tube. Proceedings of the Royal Society of London. Series A. Mathematical and Physical Sciences, 219(1137):186-203, 1953. 
[25] Rutherford Aris. On the dispersion of a solute in a fluid flowing through a tube. Proceedings of the Royal Society of London. Series A. Mathematical and Physical Sciences, 235(1200):67-77, 1956.

[26] Octave Levenspiel. Chemical reaction engineering. Wiley New York etc., 2 edition, 1972.

[27] Charles K Westbrook and Frederick L Dryer. Chemical kinetic modeling of hydrocarbon combustion. Progress in Energy and Combustion Science, 10(1):157,1984 .

[28] CH Gibson and CN Hinshelwood. The homogeneous reaction between hydrogen and oxygen. Proceedings of the Royal Society of London. Series A, Containing Papers of a Mathematical and Physical Character, 119(783):591-606, 1928.

[29] WL Garstang and CN Hinshelwood. The kinetics of the combination of hydrogen and oxygen: The influence of iodine. Proceedings of the Royal Society of London. Series A, Containing Papers of a Mathematical and Physical Character, 130(815):640-654, 1931.

[30] GH Grant and CN Hinshelwood. The upper pressure limit in the chain reaction between hydrogen and oxygen. Proceedings of the Royal Society of London. Series A, Containing Papers of a Mathematical and Physical Character, 141(843):29-40, 1933.

[31] AH Willbourn and CN Hinshelwood. The mechanism of the hydrogen-oxygen reaction. i. the third explosion limit. Proceedings of the Royal Society of London A: Mathematical, Physical and Engineering Sciences, 185(1003):353-369, 1946.

[32] AH Willbourn and CN Hinshelwood. The mechanism of the hydrogen-oxygen reaction. ii. the reaction occurring between the second and third explosion limits. Proceedings of the Royal Society of London A: Mathematical, Physical and Engineering Sciences, 185(1003):369-376, 1946.

[33] Harold R Heiple and Bernard Lewis. The reaction between hydrogen and oxygen: kinetics of the third explosion limit. The Journal of Chemical Physics, 9(8):584-590, 1941.

[34] Guenther Von Elbe and Bernard Lewis. Mechanism of the thermal reaction between hydrogen and oxygen. The Journal of Chemical Physics, 10(6):366393, 1942.

[35] Marcus Ó Conaire, Henry J Curran, John M Simmie, William J Pitz, and Charles K Westbrook. A comprehensive modeling study of hydrogen oxidation. International journal of chemical kinetics, 36(11):603-622, 2004.

[36] Juan Li, Zhenwei Zhao, Andrei Kazakov, and Frederick L Dryer. An updated comprehensive kinetic model of hydrogen combustion. International journal of chemical kinetics, 36(10):566-575, 2004. 
[37] Priyank Saxena and Forman A Williams. Testing a small detailed chemicalkinetic mechanism for the combustion of hydrogen and carbon monoxide. Combustion and Flame, 145(1):316-323, 2006.

[38] Zekai Hong, David F Davidson, and Ronald K Hanson. An improved h2/o2 mechanism based on recent shock tube/laser absorption measurements. Combustion and Flame, 158(4):633-644, 2011.

[39] Michael P Burke, Marcos Chaos, Yiguang Ju, Frederick L Dryer, and Stephen J Klippenstein. Comprehensive h2/o2 kinetic model for high-pressure combustion. International Journal of Chemical Kinetics, 44(7):444-474, 2012.

[40] DB Olson and WC Gardiner Jr. An evaluation of methane combustion mechanisms. The Journal of Physical Chemistry, 81(25):2514-2519, 1977.

[41] KJ Hughes, T Turanyi, AR Clague, and MJ Pilling. Development and testing of a comprehensive chemical mechanism for the oxidation of methane. International Journal of Chemical Kinetics, 33(9):513-538, 2001.

[42] G. P. Smith, D. M. Golden, M. Frenklach, B. Eiteener, M. Goldenberg, C. T. Bowman, R. K. Hanson, W. C. Gardiner, V. V. Lissianski, and Z. W. Qin. GRI-Mech 3.0. http://www.me. berkeley.edu/gri_mech/, 2000.

[43] Craig T Bowman. A shock-tube investigation of the high-temperature oxidation of methanol. Combustion and Flame, 25:343-354, 1975.

[44] Juan Li, Zhenwei Zhao, Andrei Kazakov, Marcos Chaos, Frederick L Dryer, and James J Scire. A comprehensive kinetic mechanism for co, ch2o, and ch3oh combustion. International Journal of Chemical Kinetics, 39(3):109-136, 2007.

[45] Philippe Dagaut, Michel Cathonnet, and Jean-claude Boettner. Kinetics of ethane oxidation. International Journal of Chemical Kinetics, 23(5):437-455, 1991.

[46] Chitralkumar V Naik and Anthony M Dean. Modeling high pressure ethane oxidation and pyrolysis. Proceedings of the Combustion Institute, 32(1):437-443, 2009 .

[47] Philippe Dagaut, Jean-Claude Boettner, and Michel Cathonnet. Ethylene pyrolysis and oxidation: A kinetic modeling study. International journal of chemical kinetics, 22(6):641-664, 1990.

[48] Juan Li, Andrei Kazakov, and Frederick L Dryer. Ethanol pyrolysis experiments in a variable pressure flow reactor. International Journal of Chemical Kinetics, 33(12):859-867, 2001.

[49] Kun Wang, Stephanie M Villano, and Anthony M Dean. Fundamentally-based kinetic model for propene pyrolysis. Combustion and Flame, 162(12):4456-4470, 2015. 
[50] Maria V Petrova and Forman A Williams. A small detailed chemical-kinetic mechanism for hydrocarbon combustion. Combustion and Flame, 144(3):526$544,2006$.

[51] Charles K Westbrook, Jürgen Warnatz, and William J Pitz. A detailed chemical kinetic reaction mechanism for the oxidation of iso-octane and n-heptane over an extended temperature range and its application to analysis of engine knock. Symposium (international) on combustion, 22(1):893-901, 1989.

[52] Henry J Curran, Paolo Gaffuri, William J Pitz, and Charles K Westbrook. A comprehensive modeling study of $\mathrm{n}$-heptane oxidation. Combustion and flame, 114(1):149-177, 1998.

[53] LJ Spadaccini and MB Colket. Ignition delay characteristics of methane fuels. Progress in Energy and Combustion Science, 20(5):431-460, 1994.

[54] CJ Brown and GO Thomas. Experimental studies of shock-induced ignition and transition to detonation in ethylene and propane mixtures. Combustion and Flame, 117(4):861-870, 1999.

[55] Yoshiaki Hidaka, Toshihide Nishimori, Kazutaka Sato, Yusuke Henmi, Rieko Okuda, Koji Inami, and Tetsuo Higashihara. Shock-tube and modeling study of ethylene pyrolysis and oxidation. Combustion and Flame, 117(4):755-776, 1999 .

[56] Michael Frenklach and David E Bornside. Shock-initiated ignition in methanepropane mixtures. Combustion and flame, 56(1):1-27, 1984.

[57] Sinéad M Burke, Ultan Burke, Reuben Mc Donagh, Olivier Mathieu, Irmis Osorio, Charles Keesee, Anibal Morones, Eric L Petersen, Weijing Wang, Trent A DeVerter, et al. An experimental and modeling study of propene oxidation. part 2: Ignition delay time and flame speed measurements. Combustion and Flame, 162(2):296-314, 2015.

[58] Sung Hyun Pyun, Wei Ren, David F Davidson, and Ronald K Hanson. Methane and ethylene time-history measurements in n-butane and n-heptane pyrolysis behind reflected shock waves. Fuel, 108:557-564, 2013.

[59] Ivo Stranic, Sung Hyun Pyun, David Frank Davidson, and Ronald Kenneth Hanson. Multi-species measurements in 2-butanol and i-butanol pyrolysis behind reflected shock waves. Combustion and Flame, 160(6):1012-1019, 2013.

[60] FN Egolfopoulos and CK Law. An experimental and computational study of the burning rates of ultra-lean to moderately-rich h2/o2/n2 laminar flames with pressure variations. Symposium (international) on combustion, 23(1):333-340, 1991. 
[61] FN Egolfopoulos, DL Zhu, and CK Law. Experimental and numerical determination of laminar flame speeds: Mixtures of c 2-hydrocarbons with oxygen and nitrogen. Symposium (International) on Combustion, 23(1):471-478, 1991.

[62] MI Hassan, KT Aung, OC Kwon, and GM Faeth. Properties of laminar premixed hydrocarbon/air flames at various pressures. Journal of Propulsion and Power, 14(4):479-488, 1998.

[63] G Jomaas, XL Zheng, DL Zhu, and CK Law. Experimental determination of counterflow ignition temperatures and laminar flame speeds of c 2-c 3 hydrocarbons at atmospheric and elevated pressures. Proceedings of the Combustion Institute, 30(1):193-200, 2005.

[64] M Cathonnet, JC Boettner, and H James. Experimental study and numerical modeling of high temperature oxidation of propane and n-butane. Symposium (International) on Combustion, 18(1):903-913, 1981.

[65] P Dagaut, M Cathonnet, JP Rouan, R Foulatier, A Quilgars, JC Boettner, $\mathrm{F}$ Gaillard, and $\mathrm{H}$ James. A jet-stirred reactor for kinetic studies of homogeneous gas-phase reactions at pressures up to ten atmospheres. Journal of Physics E: Scientific Instruments, 19(3):207-209, 1986.

[66] P Dagaut, M Cathonnet, JC Boettner, and F Gaillard. Kinetic modeling of ethylene oxidation. Combustion and flame, 71(3):295-312, 1988.

[67] Sinéad M Burke, Wayne Metcalfe, Olivier Herbinet, Frédérique Battin-Leclerc, Francis M Haas, Jeffrey Santner, Frederick L Dryer, and Henry J Curran. An experimental and modeling study of propene oxidation. part 1: Speciation measurements in jet-stirred and flow reactors. Combustion and Flame, 161(11):2765-2784, 2014.

[68] Anuj Bhargava and Phillip R Westmoreland. Measured flame structure and kinetics in a fuel-rich ethylene flame. Combustion and Flame, 113(3):333-347, 1998.

[69] H. Wang, E. Dames, B. Sirjean, D. A. Sheen, R. Tangko, A. Violi, J. Y. W. Lai, F. N. Egolfopoulos, D. F. Davidson, R. K. Hanson, C. T. Bowman, C. K. Law, W. Tsang, N. P. Cernansky, D. L. Miller, and R. P. Lindstedt. A high-temperature chemical kinetic model of n-alkane (up to n-dodecane), cyclohexane, and methyl-, ethyl-, n-propyl and n-butyl-cyclohexane oxidation at high temperatures, jetsurf version 2.0. http://web.stanford.edu/group/ haiwanglab/JetSurF/JetSurF2.0/index.html, 2010.

[70] Hai Wang, Xiaoqing You, Ameya V. Joshi, Scott G. Davis, Alexander Laskin, Fokion Egolfopoulos, and Chung K. Law. Usc mech version ii. high-temperature combustion reaction model of h2/co/c1-c4 compounds. http://ignis.usc. edu/USC_Mech_II.htm, 2007. 
[71] RS Tranter, A Raman, R Sivaramakrishnan, and K Brezinsky. Ethane oxidation and pyrolysis from 5 bar to 1000 bar: Experiments and simulation. International journal of chemical kinetics, 37(5):306-331, 2005.

[72] Robert S Tranter, Raghu Sivaramakrishnan, Kenneth Brezinsky, and Mark D Allendorf. High pressure, high temperature shock tube studies of ethane pyrolysis and oxidation. Physical Chemistry Chemical Physics, 4(11):2001-2010, 2002.

[73] Chong-Wen Zhou, Yang Li, Eoin O'Connor, Kieran P Somers, Sébastien Thion, Charles Keesee, Olivier Mathieu, Eric L Petersen, Trent A DeVerter, Matthew A Oehlschlaeger, Goutham Kukkadapu, Chih-Jen Sung, Majed Alrefae, Fathi Khaled, Aamir Farooq, Patricia Dirrenberger, Pierre-Alexandre Glaude, Frdrique Battin-Leclerc, Jeffrey Santner, Yiguang Ju, Timothy Held, Francis M. Haas, Frederick L. Dryer, and Henry J. Curran. A comprehensive experimental and modeling study of isobutene oxidation. Combustion and Flame, 167:353-379, 2016.

[74] Matthew A Oehlschlaeger, David F Davidson, and Ronald K Hanson. Hightemperature thermal decomposition of isobutane and n-butane behind shock waves. J. Phys. Chem. A, 108(19):4247-4253, 2004.

[75] E Ranzi, A Frassoldati, R Grana, A Cuoci, T Faravelli, AP Kelley, and CK Law. Hierarchical and comparative kinetic modeling of laminar flame speeds of hydrocarbon and oxygenated fuels. Progress in Energy and Combustion Science, 38(4):468-501, 2012.

[76] Amir Mze-Ahmed, Kamal Hadj-Ali, Philippe Dagaut, and Guillaume Dayma. Experimental and modeling study of the oxidation kinetics of n-undecane and n-dodecane in a jet-stirred reactor. Energy \&3 Fuels, 26(7):4253-4268, 2012.

[77] Stephen Dooley, Sang Hee Won, Joshua Heyne, Tanvir I Farouk, Yiguang Ju, Frederick L Dryer, Kamal Kumar, Xin Hui, Chih-Jen Sung, Haowei Wang, et al. The experimental evaluation of a methodology for surrogate fuel formulation to emulate gas phase combustion kinetic phenomena. Combustion and Flame, 159(4):1444-1466, 2012.

[78] Tomasz Malewicki, Soumya Gudiyella, and Kenneth Brezinsky. Experimental and modeling study on the oxidation of jet a and the n-dodecane/iso-octane/npropylbenzene/1, 3, 5-trimethylbenzene surrogate fuel. Combustion and Flame, 160(1):17-30, 2013.

[79] Yangye Zhu, Sijie Li, David F Davidson, and Ronald K Hanson. Ignition delay times of conventional and alternative fuels behind reflected shock waves. Proceedings of the Combustion Institute, 35(1):241-248, 2015. 
[80] Philippe Dagaut and Michel Cathonnet. The ignition, oxidation, and combustion of kerosene: A review of experimental and kinetic modeling. Progress in energy and combustion science, 32(1):48-92, 2006.

[81] Hai Wang. personal communication.

[82] Henry J Curran, P Gaffuri, WJ Pitz, and CK Westbrook. A comprehensive modeling study of iso-octane oxidation. Combustion and flame, 129(3):253$280,2002$.

[83] Alison S Tomlin. The role of sensitivity and uncertainty analysis in combustion modelling. Proceedings of the Combustion Institute, 34(1):159-176, 2013.

[84] Hai Wang and David A Sheen. Combustion kinetic model uncertainty quantification, propagation and minimization. Progress in Energy and Combustion Science, 47:1-31, 2015.

[85] IM Sobol'. On the systematic search in a hypercube. SIAM Journal on Numerical Analysis, 16(5):790-793, 1979.

[86] Tilo Ziehn. Development and application of global sensitivity analysis methods in environmental and safety engineering. PhD thesis, University of Leeds, 2008.

[87] DL Baulch, CT Bowman, CJ Cobos, RA Cox, Th Just, JA Kerr, MJ Pilling, D Stocker, J Troe, W Tsang, RW Walker, and J Warnatz. Evaluated kinetic data for combustion modeling: supplement ii. Journal of physical and chemical reference data, 34(3):757-1397, 2005.

[88] DL Baulch, CJ Cobos, RA Cox, C Esser, P Frank, Th Just, JA Kerr, MJ Pilling, J Troe, RW Walker, and J Warnatz. Evaluated kinetic data for combustion modelling. Journal of Physical and Chemical Reference Data, 21(3):411-734, 1992.

[89] David A Sheen, Xiaoqing You, Hai Wang, and Terese Løvås. Spectral uncertainty quantification, propagation and optimization of a detailed kinetic model for ethylene combustion. Proceedings of the Combustion Institute, 32(1):535$542,2009$.

[90] Tianfeng Lu and Chung K Law. Toward accommodating realistic fuel chemistry in large-scale computations. Progress in Energy and Combustion Science, 35(2):192-215, 2009.

[91] S Vajda, P Valko, and Tamas Turanyi. Principal component analysis of kinetic models. International Journal of Chemical Kinetics, 17(1):55-81, 1985.

[92] Tianfeng Lu and Chung K Law. A directed relation graph method for mechanism reduction. Proceedings of the Combustion Institute, 30(1):1333-1341, 2005 . 
[93] G Esposito and HK Chelliah. Skeletal reaction models based on principal component analysis: Application to ethylene-air ignition, propagation, and extinction phenomena. Combustion and Flame, 158(3):477-489, 2011.

[94] Hrvoje Jasak, Aleksandar Jemcov, and Zeljko Tukovic. Openfoam: A c++ library for complex physics simulations. In International workshop on coupled methods in numerical dynamics, IUC, Dubrovnik, Croatia, 2007.

[95] RF Johnson, AC VanDine, GL Esposito, and HK Chelliah. On the axisymmetric counterflow flame simulations: Is there an optimal nozzle diameter and separation distance to apply quasi one-dimensional theory? Combustion Science and Technology, 187(1-2):37-59, 2015.

[96] Forman A. Williams. Combustion Theory. Addison-Wesley, 1985.

[97] Adela Ben-Yakar and Ronald K Hanson. Cavity flame-holders for ignition and flame stabilization in scramjets: an overview. Journal of Propulsion and Power, 17(4):869-877, 2001.

[98] Jürgen Warnatz, Ulrich Maas, Robert W Dibble, and J Warnatz. Combustion, volume 3. Springer, 2001.

[99] Ulrich Maas and Stephen B Pope. Simplifying chemical kinetics: intrinsic lowdimensional manifolds in composition space. Combustion and flame, 88(3):239264, 1992.

[100] Norbert Peters. Turbulent Combustion. Cambridge university press, 2000.

[101] Robert D Rockwell Jr, Christopher P Goyne, Brian E Rice, Toshinori Kouchi, James C McDaniel, and Jack R Edwards. Collaborative experimental and computational study of a dual-mode scramjet combustor. Journal of Propulsion and Power, 30(3):530-538, 2014.

[102] Benjamin J Tatman, Robert D Rockwell, Chris P Goyne, James C McDaniel, and James M Donohue. Experimental study of vitiation effects on flameholding in a cavity flameholder. Journal of Propulsion and Power, 29(2):417-423, 2013.

[103] S.M. Correa. Turbulence-chemistry interactions in the intermediate regime of premixed combustion. Combustion and Flame, 93(1-2):41-60, 1993.

[104] J.-Y. Chen. Stochastic modeling of partially stirred reactors. Combustion Science and Technology, 122(1-6):63-94, 1997.

[105] SB Pope. Computationally efficient implementation of combustion chemistry using in situ adaptive tabulation. Combustion Theory and Modelling, 1:41-63, 1997. 
[106] Jesse A Fulton, Jack R Edwards, Hassan A Hassan, James C McDaniel, Christopher P Goyne, Robert D Rockwell, Andrew D Cutler, Craig T Johansen, and Paul M Danehy. Large-eddy/reynolds-averaged navier-stokes simulations of reactive flow in dual-mode scramjet combustor. Journal of Propulsion and Power, 30(3):558-575, 2014.

[107] R Rockwell, C Goyne, Brian Rice, Harsha Chelliah, J McDaniel, J Edwards, Luca Cantu, Emanuela Gallo, A Cutler, and P Danehy. Development of a premixed combustion capability for dual-mode scramjet experiments. In 53rd AIAA Aerospace Sciences Meeting, Kissimmee, Florida, 2015.

[108] RH Krauss and JC McDaniel. A clean air continuous flow propulsion facility. AIAA Paper, 92:3912, 1992.

[109] Amarnatha S Potturi and Jack R Edwards. Large-eddy/reynolds-averaged navier-stokes simulation of cavity-stabilized ethylene combustion. Combustion and Flame, 162(4):1176-1192, 2015.

[110] Kiran Ramesh, Jack R Edwards, HK Chelliah, CP Goyne, JC McDaniel, RD Rockwell, Justin Kirik, AD Cutler, and PM Danehy. Large eddy simulation of high-speed, premixed ethylene combustion. In 53rd AIAA Aerospace Sciences Meeting, pages 2015-0356, 2015.

[111] Ian A Schultz, Christopher S Goldenstein, R Mitchell Spearrin, Jay B Jeffries, Ronald K Hanson, Robert D Rockwell, and Christopher P Goyne. Multispecies midinfrared absorption measurements in a hydrocarbon-fueled scramjet combustor. Journal of Propulsion and Power, 30(6):1595-1604, 2014.

[112] Zhaoyu Luo, Chun Sang Yoo, Edward S Richardson, Jacqueline H Chen, Chung K Law, and Tianfeng Lu. Chemical explosive mode analysis for a turbulent lifted ethylene jet flame in highly-heated coflow. Combustion and Flame, 159(1):265-274, 2012.

[113] MJ Rahimi, G Esposito, HK Chelliah, V Hiremath, and SB Pope. Chemical kinetic model reduction based on partially-stirred reactor simulations with comparable chemical and mixing time scales. In 51st AIAA Aerospace Sciences Meeting, Grapevine, TX, 2013.

[114] Yu Wang, Abhijeet Raj, and Suk Ho Chung. A pah growth mechanism and synergistic effect on pah formation in counterflow diffusion flames. Combustion and flame, 160(9):1667-1676, 2013.

[115] Ascher H Shapiro. The Dynamics and Thermodynamics of Compressible Fluid Flow, volume 1. The Ronald Press Company, 1953. 